\title{
1986 IUCN Red
of Threatened \\ Animals
}

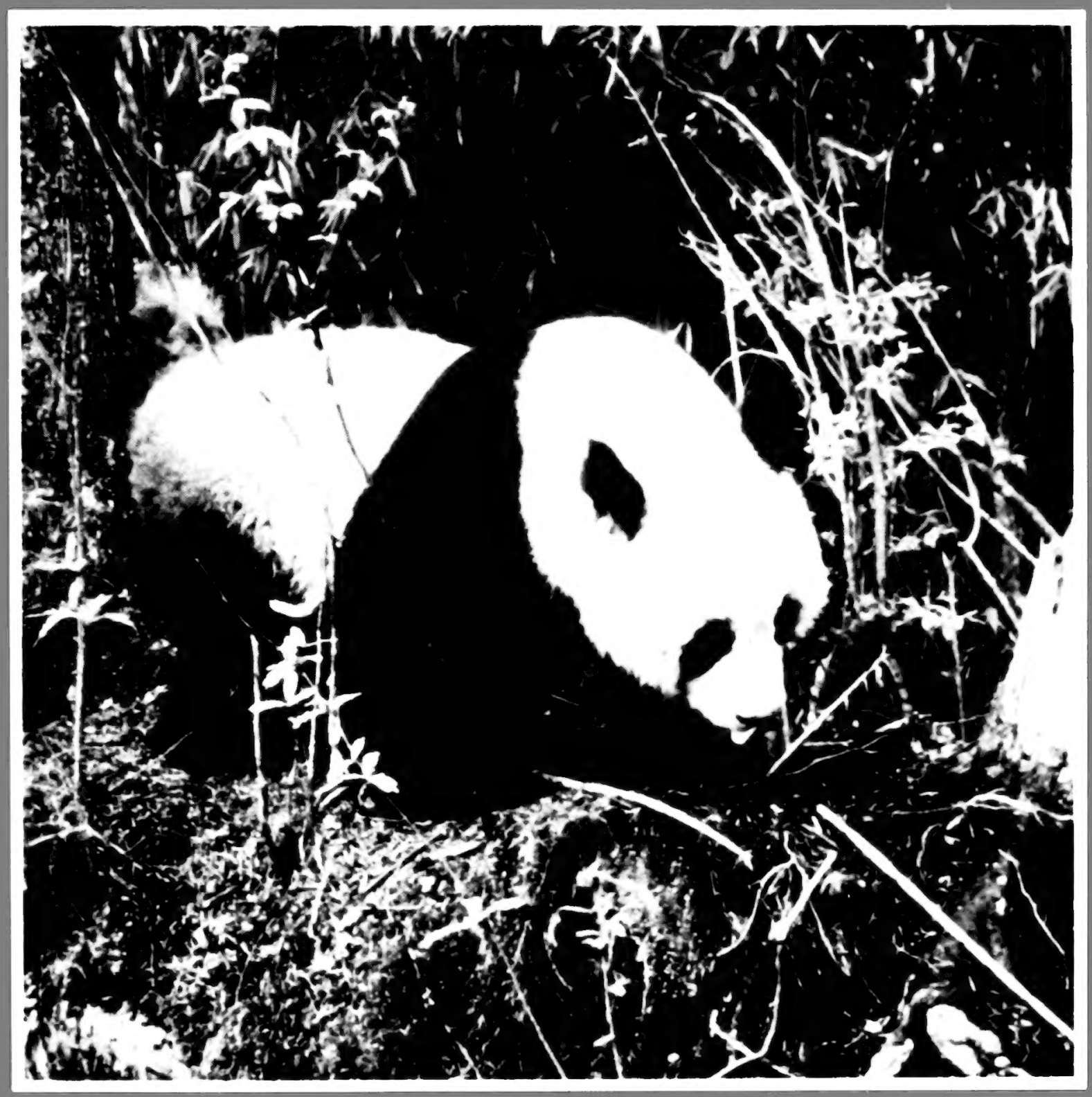


Digitized by the Internet Archive in 2010 with funding from UNEP-WCMC, Cambridge 


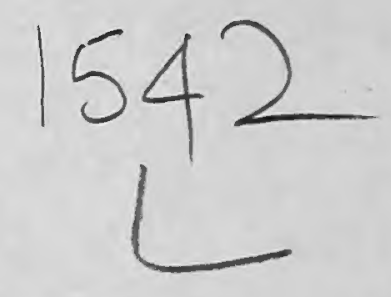

1986 IUCN RED LIST

OF THREATENED ANIMALS

\author{
Prepared by \\ The IUCN Conservation Monitoring Centre \\ Cambridge U.K.
}

International Union for Conservation of Nature and Natural Resources 
IUCN (International Union for Conservation of Nature and Natural Resources) is a network of governments, nongovernmental organizations (NGOs), scientists and other conservation experts, joined together to promote the protection and sustainable use of living resources

Founded in 1948, IUCN has more than 500 member governments and NGOs in over 116 countries. Its six Commissions consist of more than 2000 experts on threatened species, protected areas, ecology, environmental planning, environmental policy, law and administration, and environmental education.

IUCN monitors the status of ecosystems and species throughout the world; plans conservation action, both at strategic level through the World Conservation Strategy and at the programme level through its programme of conservation for sustainable development; promotes such action by governments, inter-governmental bodies and nongovernmental organisations; and provides the assistance and advice necessary to achieve such action.

THE IUCN CONSERVATION MONITORING CENTRE is the division of the Union that provides a data service to IUCN and to the conservation and development community. CMC is building an integrated and cross-referenced database on animals and plants of conservation concern, on wildlife trade, and on ecosystems (principally protected areas so far). The CMC produces a wide variety of specialist outputs and analyses, as well as major publications such as Red Data Books and Protected Area Directories. Other IUCN Centres focus on Environmental Law, and Conservation for Development. Collectively this information and expertise is used to assist IUCN members to prepare conservation programmes, and to provide information and advice to the conservation and development communities. Should you wish to consult these services please contact the:

IUCN Secretariat, World Conservation Centre Ave du Mont Blanc, $\mathrm{CH}-1196$ Gland, Switzerland

The designations of geographical entities in this book, and the presentation of the material, do not imply the expression of any opinion whatsoever on the part of IUCN concerning the legal status of any country, territory, or area, or of its authorities, or concerning the delimitation of its frontiers or boundaries.

Published by IUCN, Gland, Switzerland, and Cambridge, U.K.

Prepared with financial support from World Wildlife Fund International, and the United Nations Environment Programme, and published with a grant from World Wildlife Fund U.K.

A contribution to GEMS - the Global Environment Monitoring System.
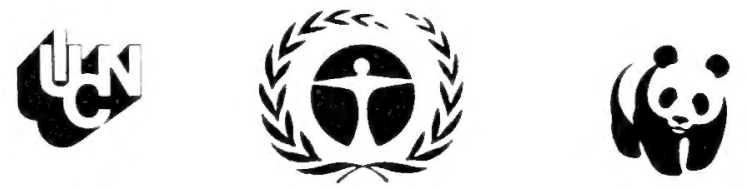

1986 International Union for Conservation of Nature and Natural Resources/United

Nations Environment Programme.

ISBN No 2-88032-605-2

Printed by Ziprint Parchment(Oxford)Ltd, 60 Hurst Street, Oxford

Cover Design by James Butler

Cover Photo WWF/GEO/Timm Rautert/BCL 


\section{PREAMBLE}

The strength of the species conservation argument is dependent on the scientific knowledge available, and the Red Data Books provide us all with a tool to be used for a restricted number of well known species or cases.

It is hoped, with this Threatened Species List, that the huge hidden information base held at the IUCN Conservation Monitoring Centre may be exposed to a wider audience. There is, of course, an inherent problem with any data base of any quality and size; everybody wants access to it instantly, but the reality is that all this hara won information has got to be distilled, manipulated and reproduced in a particular format for any user.

This takes time and time is money, so by exposing the data base one is apparently issuing an invitation to use it, whereas I think we should all be aware that to use it means we have to find the money to pay for its use. It cannot be provided for free!

The great skills of the staff and the huge network of specialists from which the data is acquired, many of whom are members of the Specialist Groups of the Species Survival Commission, are actually worth paying for, painful as this concept might be.

It was with great pleasure that World Wildlife Fund UK agreed to pay for the publication of this species list, to show what the data base holds and, to show the status of the species held, which in itself is of value to a whole range of users.

As one reviews, during this 25 th Anniversary Year of the World Wildlife Fund, what has been accomplished in species conservation, it must be said that whilst we have done well in creating an extensive information base, we are sadly still very far away from being in a position to have plans and programmes to ensure the wise management of all these threatened species. Our success in the field and 'in captivity' sometimes obscures this reality!

The reality, in fact, shows the need for greater and more vigorous action but, having said this, I believe we should congratulate the team at the Conservation Monitoring Centre for their sterling effort in the battle for species conservation.

\section{Grenville L1. Lucas,}

Chairman,

Species Survival Commission, IUCN

28th August, 1986 



\section{CONTENTS}

Introduction

Inventory Report Form

Documents on which the IUCN Red List is based

Taxonomy

IUCN Category Definitions

List of Abbreviations

Threatened Species List

Mammals

Birds

Reptiles

Amphibians

Fishes

Invertebrates
Page

iii

v

vii

viii

ix

X

2

15

33

38

39

49

97

Index 



\section{Introduction}

IUCN has long been known for the compilation of a series of Red Data Books, authoritative international inventories of threatened species. In recent years the Red Data Books have changed in format from the original loose-leaf style which allowed for frequent update of species accounts, to that of bound volumes. In addition, some books have become regional rather than global in approach, e.g. Threatened Birds of Africa and Related Islands. As a result, a list of internationally threatened species has not been readily available from IUCN for some years. This publication has been prepared in response to the increasing demand for such a list, from scientists, educationalists, zoos, the media, and conservation organisations. It is intended that new editions of the list will be published periodically.

The Red Data Book concept, originated by Sir Peter Scott in the mid-1960s, aimed to focus attention on the plight of the earth's vanishing wildlife. The concept has been immensely successful and, two decades later, many national and regional Red Dat. Books have been published; and many more countries are in the process of compiling their own threatened species lists. National and regional compilations are always likely to be more detailed than the international overview provided by IUCN. Increasingly therefore, IUCN's role will be to provide pointers to the availability of such data sources. Where these exist, IUCN will not duplicate the detailed information they contain, but will list any identified globally threatened taxa on its international list. For example, Australia has in recent years produced several texts detailing the status of its native species. IUCN will include all the relevant threatened Australian species on the international list but will not give high priority to the compilation of detailed data sheets for Australian taxa.

The next generation of Red Data Books prepared by IUCN will thus tend to be shorter, more specific publications highlighting the conservation problems of a group of species, perhaps even a single species, or an area. Such shorter compilations will allow for faster dissemination of conservation data, and will be more responsive to the changing priorities and needs of conservation. Complementing these, the periodic publication of the IUCN list of internationally threatened species will provide an index to the species data held by IUCN.

IUCN's threatened species list is compiled and maintained by the IUCN Conservation Monitoring Centre. For each threatened taxon, the list provides the scientific name, English vernacular name (where known), IUCN category (for definitions see page ix), and a brief description of the animal's distribution. This is followed by an index to the list based on the order, family and generic listing. Vernacular names for major groupings such as monkeys, parrots, and butterflies are also included.

Data upon which the listings are made are gathered from literature searches, and from correspondence with specialists around the world, including those belonging to the Specialist Groups of IUCN's Species Survival Commission. 
The number of threatened animal taxa identified by IUCN is 3117 , comprised of 385 mammals, 428 birds, 143 reptiles, 46 amphibians, 286 fishes, and 1829 invertebrates. These numbers, however, represent only those taxa whose threatened status has been brought to the attention of IUCN; many, many more taxa, particularly invertebrates are threatened, and going extinct every year unbeknownst to the scientific and conservation communities. Thus the number of threatened taxa identified by IUCN represents only the visible fraction of a much greater problem. The vertebrate listings are based almost entirely on the IUCN Red Data Books. For invertebrates, however, it must be stressed that the list is only provisional, since most species have not yet been subjected to the same review process as has been carried out for the vertebrate groups, apart from those listed in the IUCN Invertebrate Red Data Book and Swallowtail Butterfly Red Data Book. Other invertebrate listings have been taken from correspondence and a number of published sources (noted on page vii).

The threatened species list is constantly under review; as new areas or species groups are surveyed, species may be listed or delisted or categories may change. Since at any one time work is focused on a particular group of species, some parts of the list are more up to date than others. For example, a review of the conservation status of the birds of Africa and related islands was undertaken by IUCN and ICBP (the International Council for Bird Preservation) between 1982 and 1985 , leading to the publication of the Bird Red Data Book for Africa. Work is now in progress to assess the conservation status of the birds of the Americas. Information on Asian birds has not therefore been reviewed for some time (although files and correspondence are maintained), and so changes are undoubtedly required.

The need for new information is thus continuous and assistance in providing accurate data always welcome. An inventory report form is detailed on page $\mathrm{v}$ and is a guide to the information required by IUCN to assess the international conservation status of a species.

The present list undoubtedly includes a number of errors and omissions, but it is to be hoped that these will stimulate people to send new information to:-

The Species Conservation Monitoring Unit IUCN Conservation Monitoring Centre

219c Huntingdon Road

Cambridge CB3 ODL

United Kingdom 


\section{INVENTORY REPORT FORM}

Report to be mailed to SCMU, IUCN Conservation Monitoring Centre, 219(c) Huntingdon Rd, Cambridge, CB3 ODL, U.K.

\section{Country}

2. Date

3. Reporter Name:

Address:

4. Taxon

Scientific Name: Common Name:

\section{Distribution}

Present:

Former:

If possible, please include a map. Is present range preferred or enforced habitat?

6. Population

Estimated numbers in the wild. Indicate date of estimate and describe method of estimation. Are numbers increasing, decreasing or stable?

7. Habitat and Ecology

Biome type. Elevation range. Brief notes about social structure, feeding habits and diet, reproduction (gestation, breeding season, number of young, age of sexual maturation), longevity etc.

8. Scientific interest and potential value

Use in scientific or medical research; commercial value in trade or local economy.

9. Threats to Survival

Eg. habitat destruction, over-exploitation, hybridization, natural disasters, competition for food.

10. Conservation Measures Taken

Legal measures (international conventions, national laws); is law enforced? Protected areas - does it occur in national parks, reserves etc.? If so, please list. Management programmes or research programmes in progress. 
11. Conservation Measures Proposed

Same as for 10 , but measures that are needed for the conservation of the taxon.

12. Captive Breeding

Numbers in captivity. Does it breed readily in captivity? Where and when?

13. Remarks

Reference citations for description of animal. Comments about related taxa. If the above information concerns a subspecies then brief information should be given about the distribution and status of the species as a whole. Special acknowledgements etc.

\section{References}

Can be published papers, unpublished manuscripts, or references to correspondence (cited as $\underline{\text { In litt.). }}$. 


\section{DOCUMENTS ON WHICH THE IUCN RED LIST IS BASED}

The asterisk indicates books currently available from IUCN.

\section{$\underline{\text { Mammals }}$}

Goodwin, H.A. and Holloway, C.W. (1972). Red Data Book, Vol. 1 - Mammalia. Second Edition. IUCN, Morges. (Revisions: 1973 and 1974 by Goodwin, H.A. and Holloway, C.W.; 1976 by Fitter, R.S.R. and Holloway, C.W.; 1978 by Thornback, J.). Thornback, J. and Jenkins, M. (1982). Terrestrial Mammals. Part VII. In: Threatened Species of Wild Flora and Vertebrate Fauna. Report to the Commission of the European Communities. The Nature Conservancy Council, Great Britain.

Thornback, J. (1984). Wild Cattle, Bison and Buffaloes; their status and potential value. Report to UNEP. IUCN Conservation Monitoring Centre.

*Thornback, J. and Jenkins, M. (1982). The IUCN Mammal Reci Data Book. Part 1. The Americas and Australasia. IUCN, Gland.

\section{$\underline{\text { Birds }}$}

King, W.B. (1981). Endangered Birds of the World. The ICBP Bird Red Data Book. Smithsonian Institution Press and ICBP, Washington D.C.

*Collar, N.J. and Stuart, S.N. (1985). Threatened Birds of Africa and Related Islands. The ICBP/IUCN Red Data Book, Part 1. Third Edition. ICBP and IUCN, Cambridge.

\section{Amphibians and Reptiles}

*Groombridge, B. (1982). The IUCN Amphibia-Reptilia Red Data Book. Part 1, Testudines. Crocodylia, Rhynchocephalia. IUCN, Gland.

Groombridge, B. (1982). Amphibians and Reptiles. Part IV. In: Threatened Species of Wild Flora and Vertebrate Fauna. Report to the Commission of the European Communities. The Nature Conservancy Council, Great Britain.

Honneger, R. (1975/1979). Red Data Book, Vol. 3 .. Amphibia and Reptilia. Second Edition. IUCN, Gland.

\section{Fishes}

*Evans, D. (1981). Threatened Fish of Sri Lanka. IUCN Conservation Monitoring Centre, Cambridge.

Evans, D. and Almada-Villela, P. (Unpublished). Threatened Fishes of North America. Draft Document. IUCN Conservation Monitoring Centre.

Miller, R.R. (1977). Red Data Book, Vol. 4 - Pisces. Second Edition. IUCN, Morges. U.S. Department of the Interior (1986). Endangered and Threatened Wildlife and Plants. Federal Register 50 CFR 17.11 and 17.12:1-30

\section{Invertebrates}

*Collins, N.M. and Morris, M.G. (1985). Threatened Swallowtail Butterflies of the World. The IUCN Red Data Book. IUCN, Gland and Cambridge.

Collins, N.M. and Wells, S.M. (1986). Insects and other Invertebrates as Candidates for the Berne Convention. A Report to the Council of Europe. IUCN Conservation Monitoring Centre.

U.S. Department of the Interior. (1984). Endangered and Threatened Wildlife and Plants; Review of Invertebrate Wildlife for Listing as Endangered or Threatened Species. Federal Register 49(100): 21664-21675.

*Wells, S.M., Pyle, R.M. and Collins, N.M. (1983). The IUCN Invertebrate Red Data Book. IUCN, Gland. 


\section{TAXONOMY}

The systems of classification and nomenclature principally follow the undermentioned authors.

\section{$\underline{\text { Mammals }}$}

Cabrera, A. (1957-61). Catalogo de los mamiferos de America del Sur. Rev. del Mus. Argent. de Cienc. Nat. 'Bernardino Rivadavia' Cienc. Zool. 4(1,2): 1-732.

Corbet, G.B. and Hill, J.E. (1980). A World List of Mammalian Species. British Museum (Natural History) and Cornell University Press.

Hall, E.R. and Kelson, K.R. (1959). The Mammals of North America. Ronald Press Co., New York.

Honacki, J.H., Kinman, K.E. and Koeppl, J.W. (1982). Mammal Species of the World: a taxonomic and geographic reference. Allen Press Inc. and the Association of Systematics Collections, Kansas.

Meester, J. and Setzer, H.W. (Eds) (1971). The Mammals of Africa: an Identification Manual. Smithsonian Institution Press, City of Washington.

Ride, W.D.L. (1970). A Guide to the Native Mammals of Australia. Oxford University Press, Melbourne.

\section{$\underline{\text { Birds }}$}

Morony, J.J., Bock, W.J. and Farrand, J. (1975). Reference List of the Birds of the World. American Museum of Natural History, New York.

\section{$\underline{\text { Reptiles }}$}

Groombridge, B. (1983). World Checklist of Threatened Amphibians and Reptiles. Third Edition. Nature Conservancy Council, London.

Pritchard, P.C.H. (1979). Encyclopaedia of Turtles. T.F.H. Publications, New Jersey and Hong Kong.

Wermuth, H. and Mertens, R. (1977). Testudines, Crocodylia, Rhynchocephalia. Das Tierreich 100: 1-174.

\section{Amphibians}

Groombridge, B. (1983). World Checklist of Threatened Amphibians and Reptiles.

Third Edition. Nature Conservancy Council, London.

\section{Fishes}

Nelson, J.S. (1984). Fishes of the World. Second Edition. John Wiley and Sons, New York, Chichester, Brisbane, Toronto, Singapore.

Robins, C.R., Bailey, R.M., Bond, C.E., Brooker, J.R., Lachner, E.A., Lea, R.N., Scott, W.B. (1980). A List of Common and Scientific Names of Fishes from the United States and Canada. Fourth Edition. American Fisheries Society Special Publication No. 12.

\section{Invertebrates}

Hancock, D.L. (1983). Classification of the Papilionidae (Lepidoptera): a phylogenetic approach. Smithersia 2: 1-48.

Parker, S.B. (1982). Synopsis and Classification of Living Organisms. McGraw-Hill, New York. 


\section{IUCN THREATENED SPECIES CATEGORIES}

Species identified as threatened by IUCN are assigned a category indicating the degree of threat. Definitions are as follows:

\section{EXTINCT (Ex)}

Species not definitely located in the wild during the past 50 years (criterion as used by the Convention on International Trade in Endangered Species of Wild Fauna and Flora).

N.B. On a few occasions, the category Ex? has been assigned; this denotes that it is virtually certain that the taxon has recently become extinct.

\section{ENDANGERED (E)}

Taxa in danger of extinction and whose survival is unlikely if the causal factors continue operating.

Included are taxa whose numbers have been reduced to a critical level or whose habitats have been so drastically reduced that they are deemed to be in immediate danger of extinction. Also included are taxa that may be extinct but have definitely been seen in the wild in the past 50 years.

\section{VULNERABLE (V)}

Taxa believed likely to move into the 'Endangered' category in the near future if the causal factors continue operating.

Included are taxa of which most or all the populations are decreasing because of over-exploitation, extensive destruction of habitat or other environmental disturbance; taxa with populations that have been seriously depleted and whose ultimate security has not yet been assured; and taxa with populations that are still abundant but are under threat from severe adverse factors throughout their range.

N.B. In practice, 'Endangered' and 'Vulnerable' categories may include, temporarily, taxa whose populations are beginning to recover as a result of remedial action, but whose recovery is insufficient to justify their transfer to another category.

\section{RARE (R)}

Taxa with small world populations that are not at present 'Endangered' or 'Vulnerable', but are at risk.

These taxa are usually localised within restricted geographical areas or habitats or are thinly scattered over a more extensive range.

\section{INDETERMINATE (I)}

Taxa known to be 'Endangered', 'Vulnerable' or 'Rare' but where there is not enough information to say which of the three categories is appropriate. 
Taxa that are suspected but not definitely known to belong to any of the above categories, because of lack of information.

\section{THREATENED (T)}

Threatened is a general term to denote species which are 'Endangered', 'Vulnerable', 'Rare', 'Indeterminate', or 'Insufficiently Known' and should not be confused with the use of the same term by the U.S. Office of Endangered Species. In this volume it is used to identify taxa comprised of several sub-taxa which have differing status categories.

\section{COMMERCIALLY THREATENED (CT)}

Taxa not currently threatened with extinction, but most or all of whose populations are threatened as a sustainable commercial resource, or will become so, unless their exploitation is regulated.

This category applies only to taxa whose populations are assumed to be relatively large.

N.B. In practise, this category has only been used for marine species of commercial importance that are being overfished in several parts of their ranges.

\section{ABBREVIATIONS}

Arch.

Eq. Guinea

F.R.G.

I.

Is

O.

sp.

U.A.E.

U.K.

U.S.A.

U.S.S.R.

[]

\author{
Archipelago \\ Equatorial Guinea \\ Federal Republic of Germany \\ Island \\ Islands \\ Ocean \\ Unnamed species \\ United Arab Emirates \\ United Kingdom \\ United States of America \\ Union of Soviet Socialist Republics \\ Former Distribution \\ An important, introduced population
}


1986 IUCN RED LIST

OF THREATENED SPECIES 


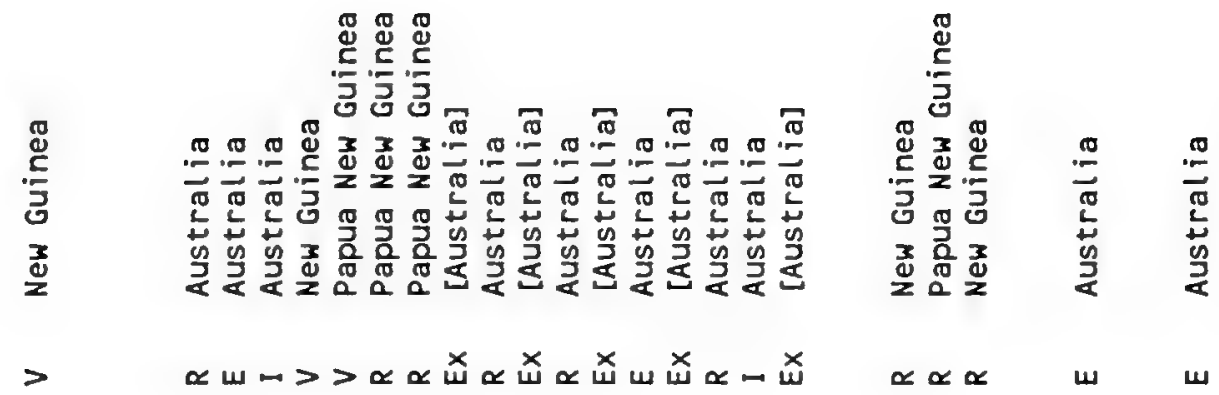

$\frac{\alpha}{0}$

8

ลิํํㅇ

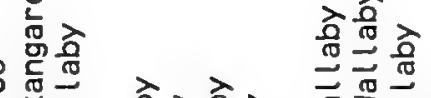

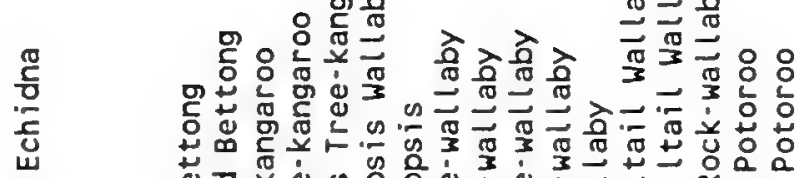

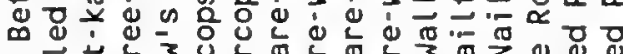

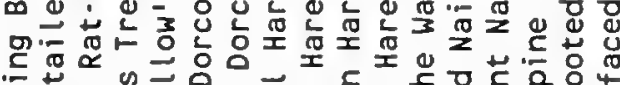

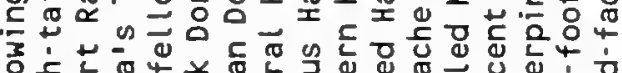

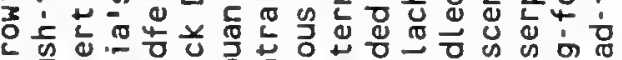

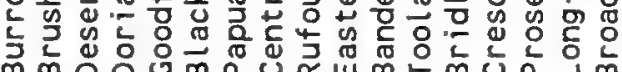

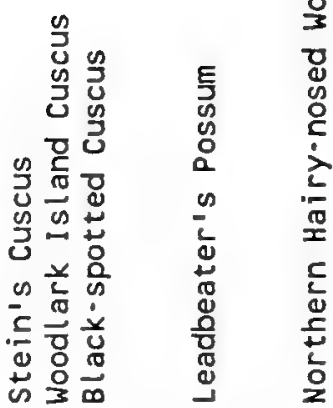

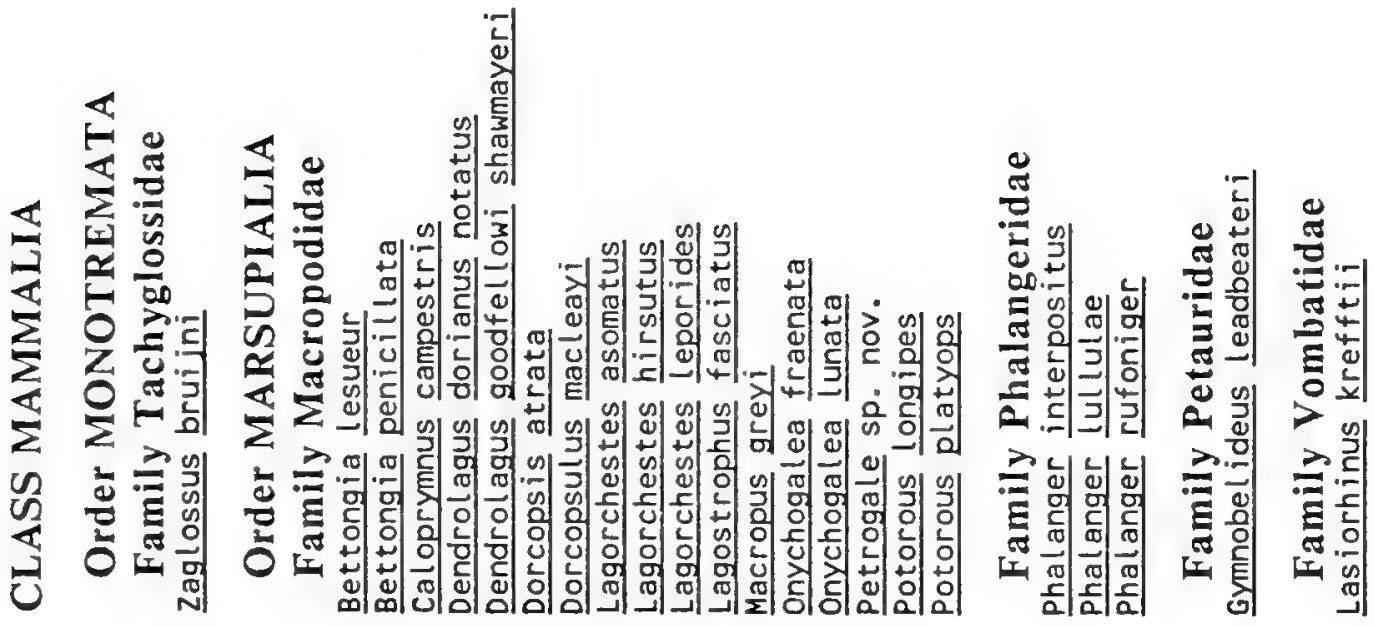




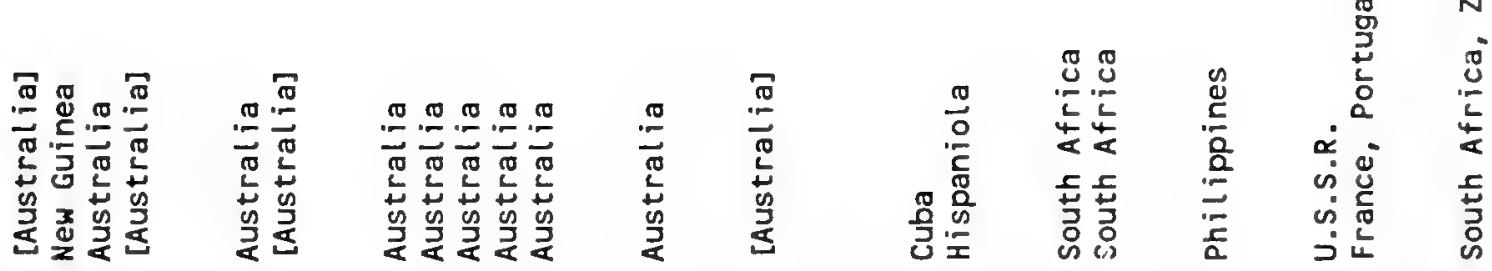

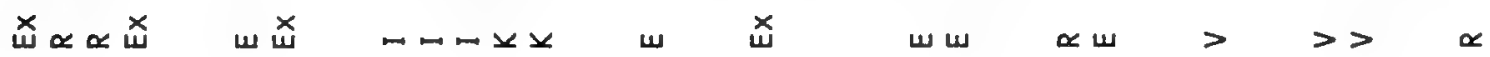

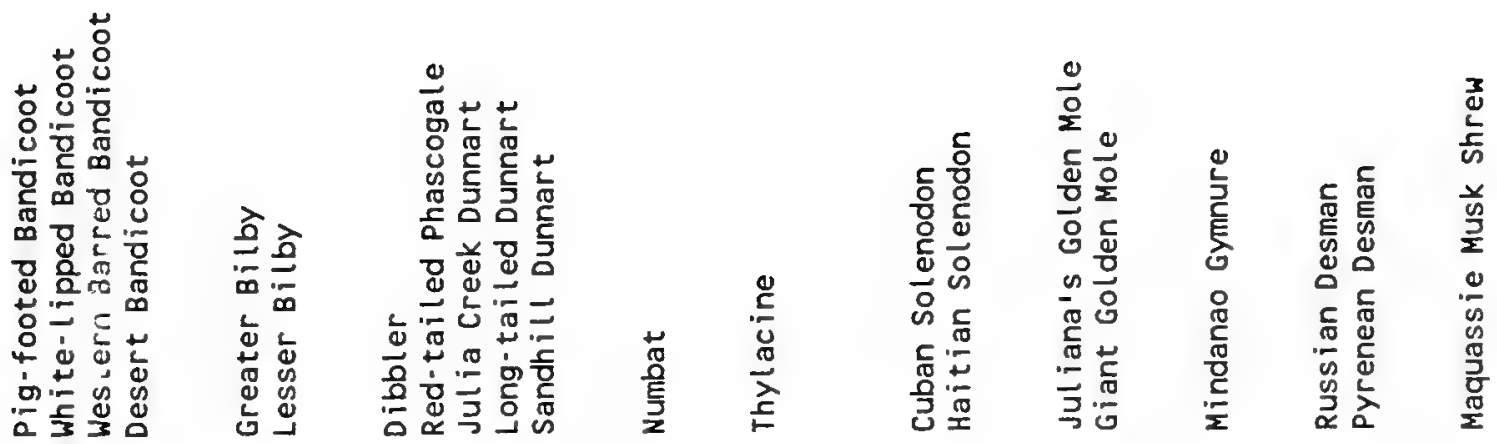
吾

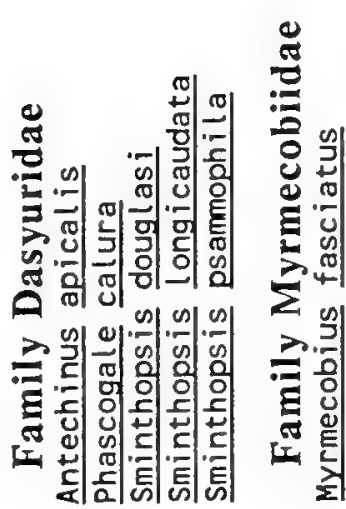

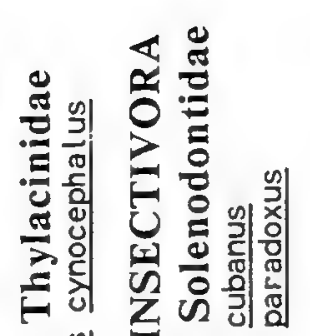

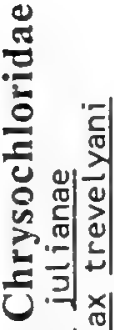
氖

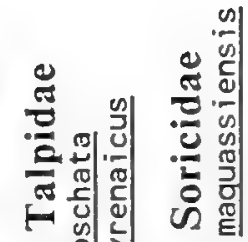

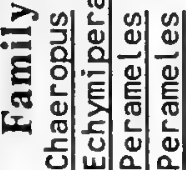

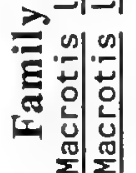

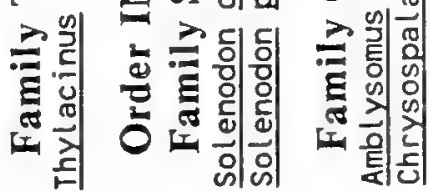

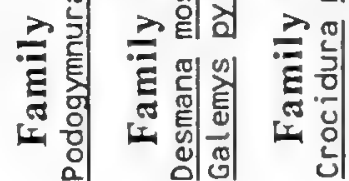




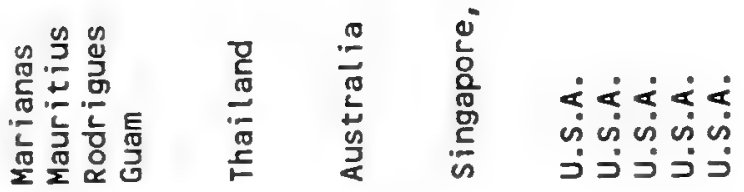

$>\propto \omega \omega \quad$ w $\quad \searrow \quad-\omega>-w$

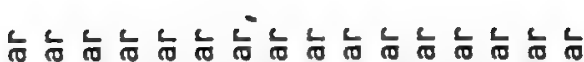
¿

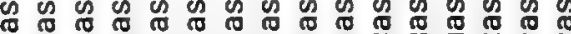

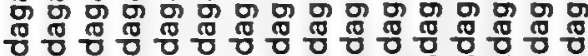

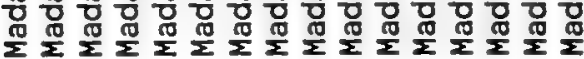

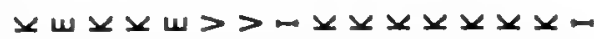
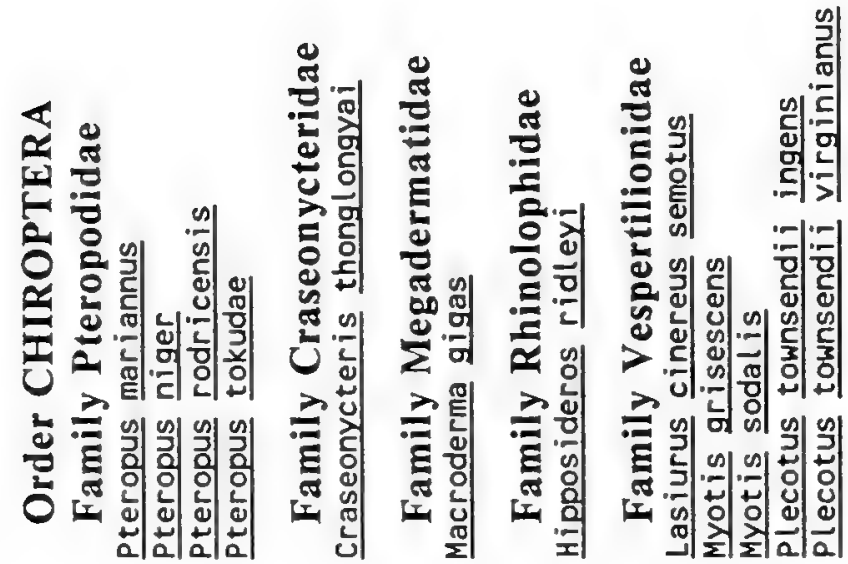

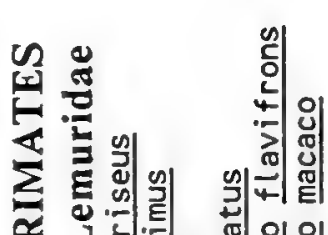

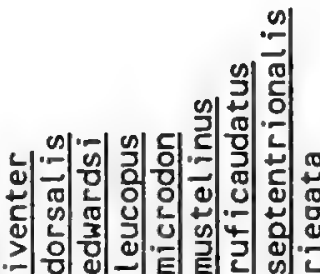

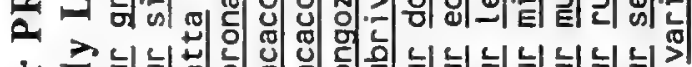

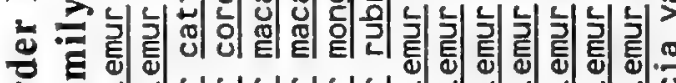

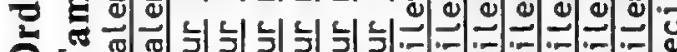

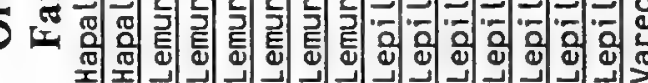


$\therefore$ 草

总总

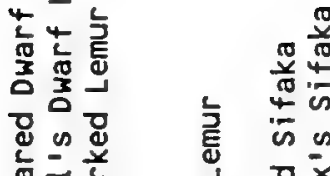

产二立

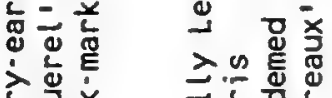

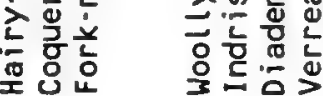

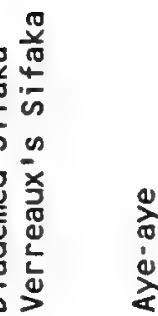

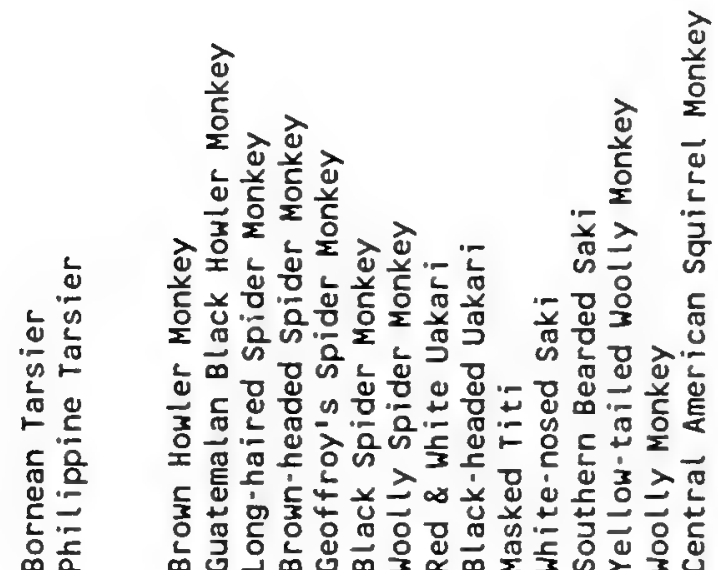

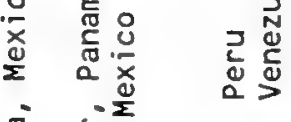

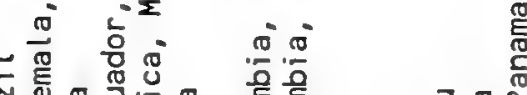

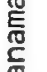

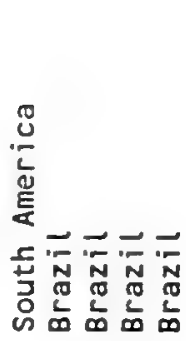

$\propto>\omega \omega>$

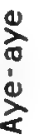

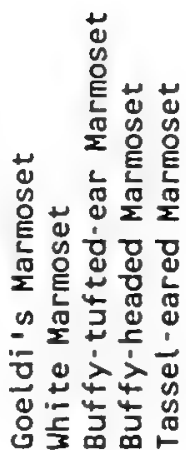
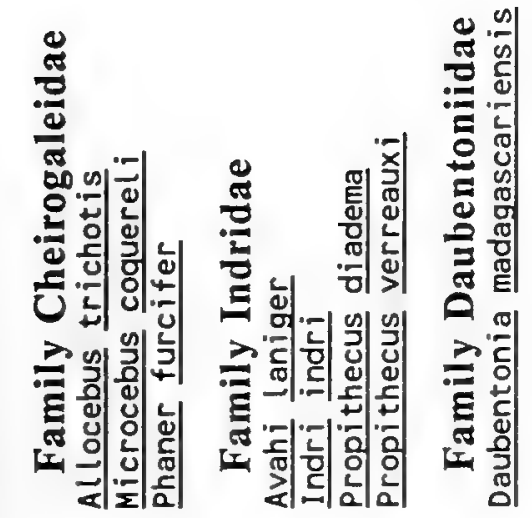

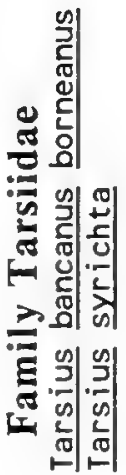
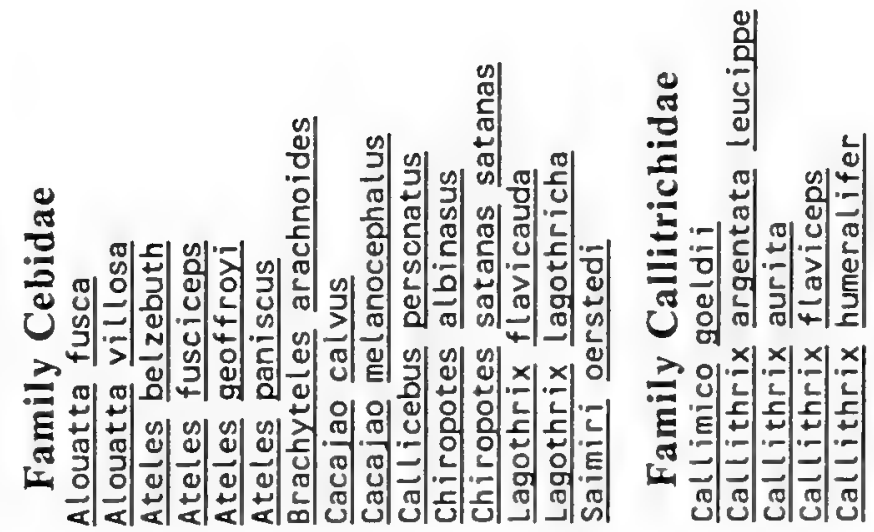
ᄃ

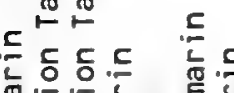

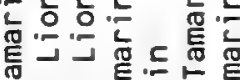

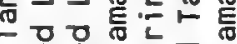

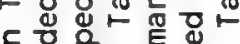
ᄃ ษ东过。 둔

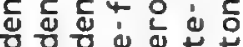

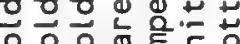

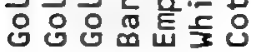

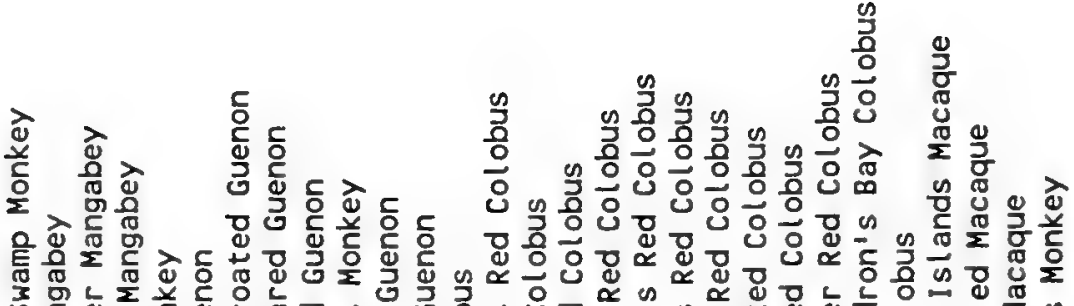

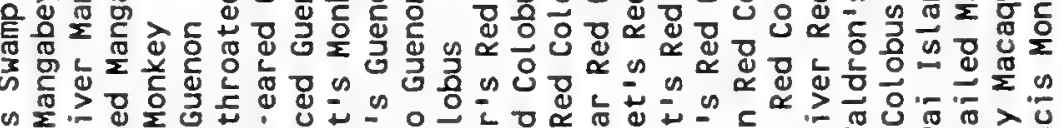

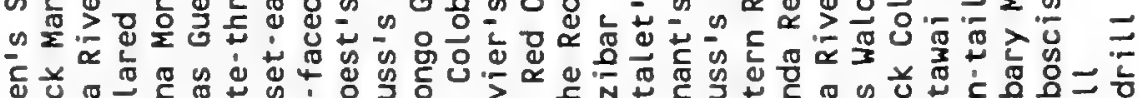

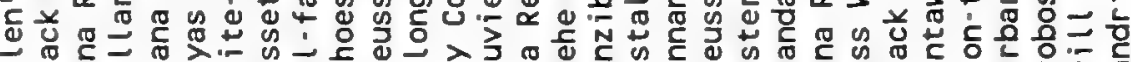

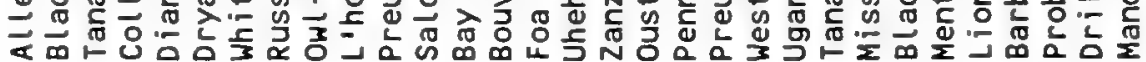

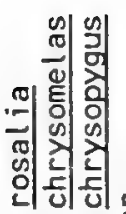

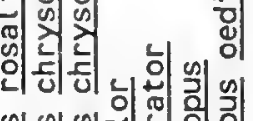

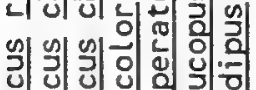
(1)

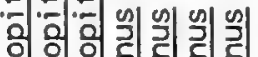

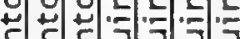

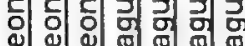

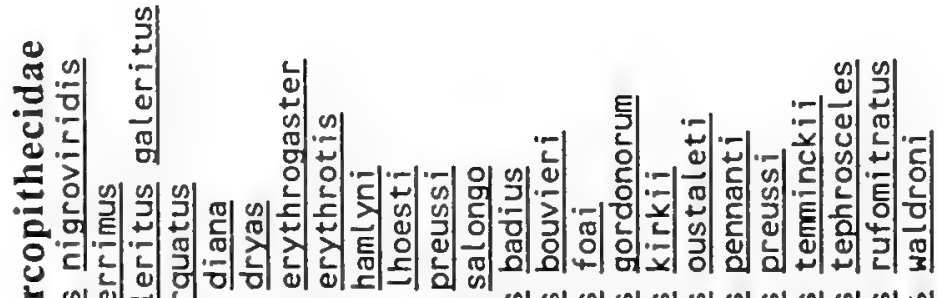

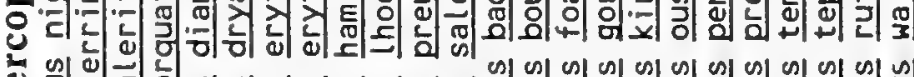

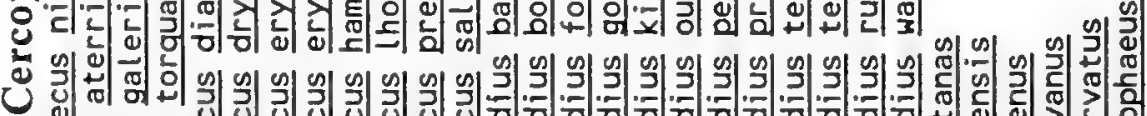

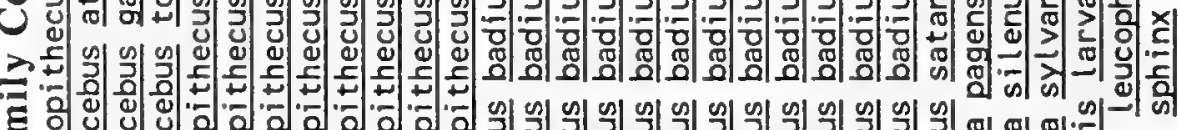

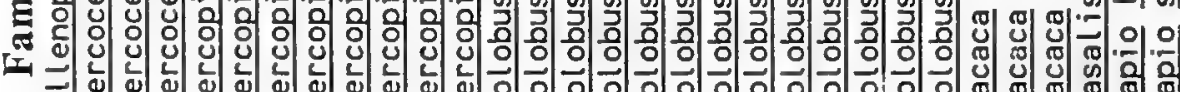
$=$ द 
$\underset{2}{>}$

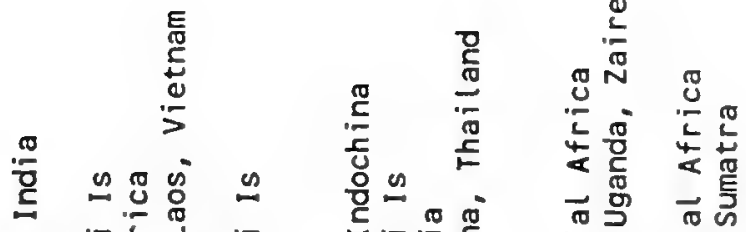

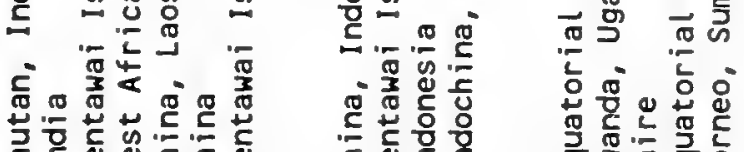

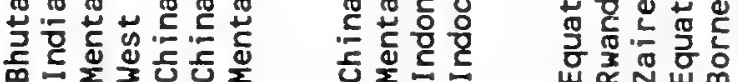

$\propto>\sim \propto \omega \propto \omega \quad m>w \omega \quad>m>>w$

宁

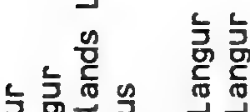

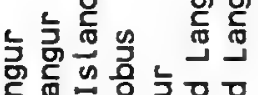

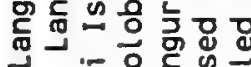
- " ร它元

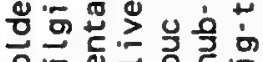

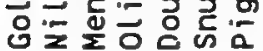

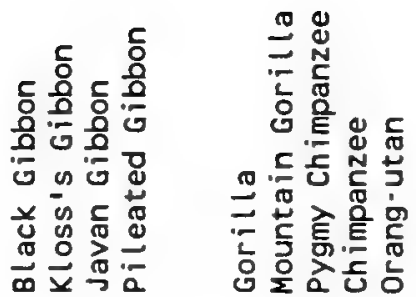

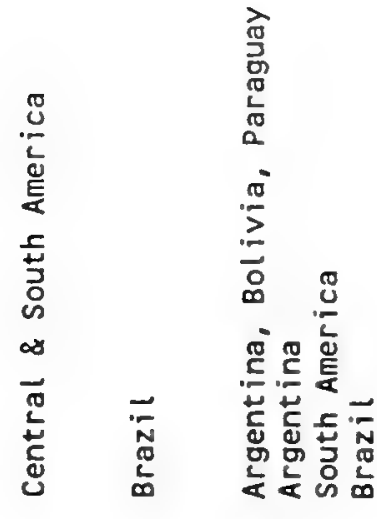




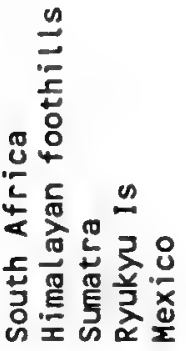

w $\propto$ $\boldsymbol{\omega}$

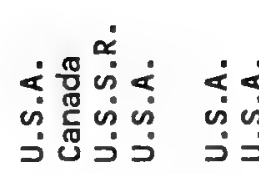
$>m>$

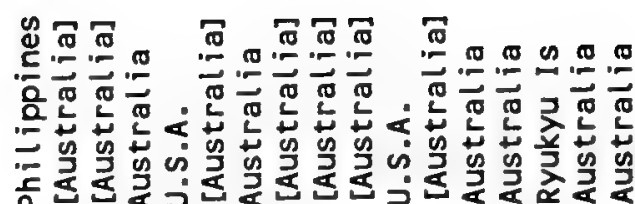

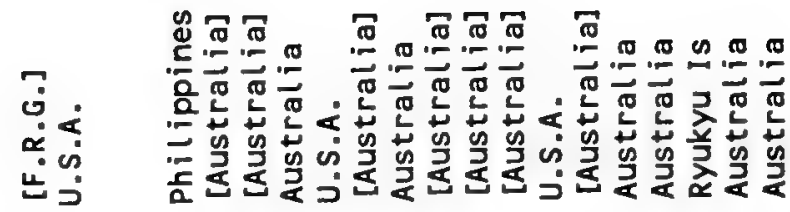

雨

w

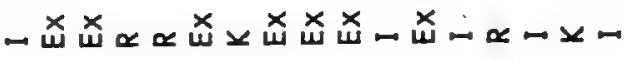

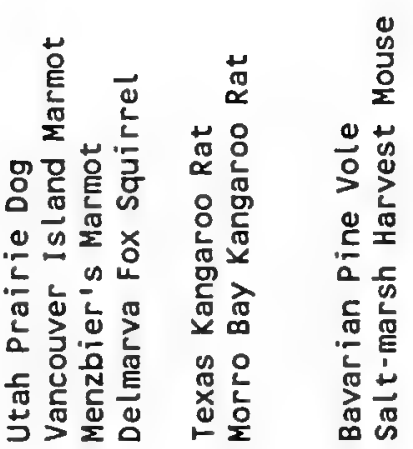

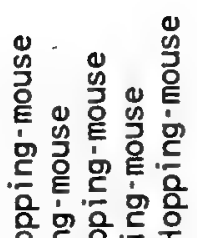

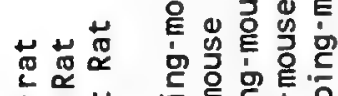

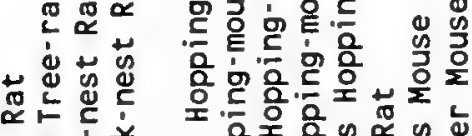

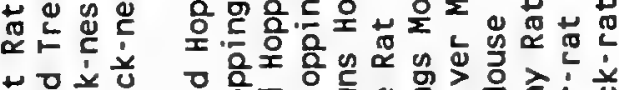

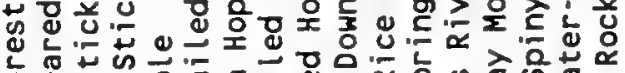

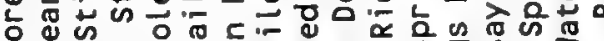

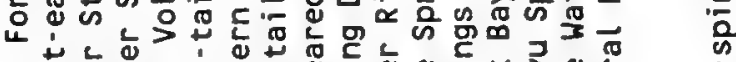

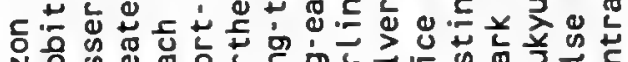

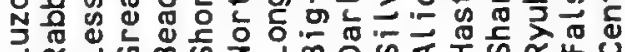
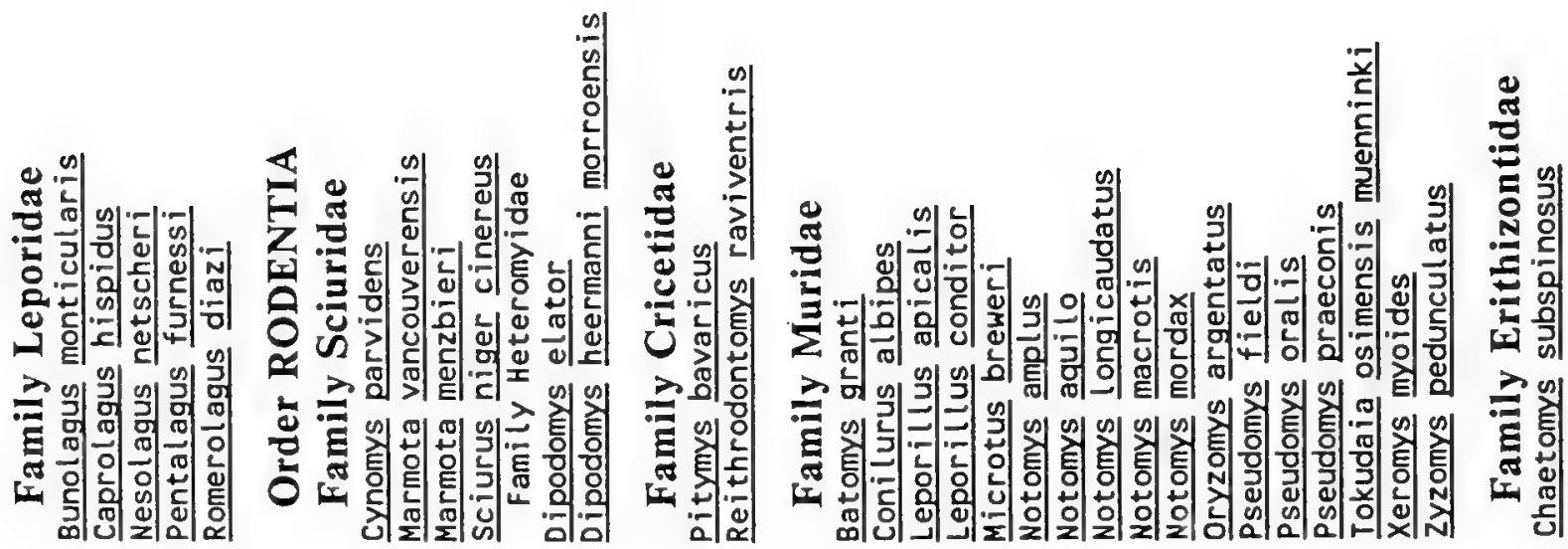


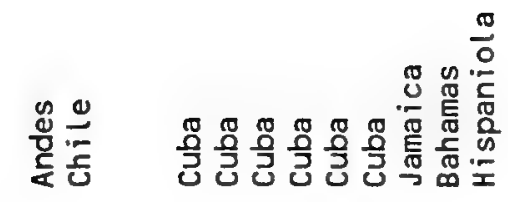

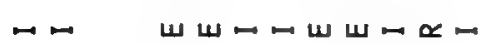

$\stackrel{0}{=}$

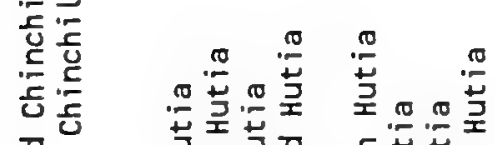

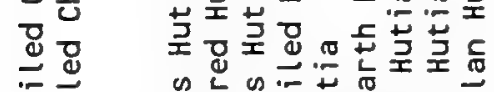

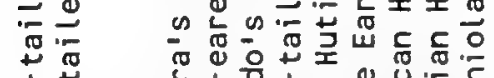

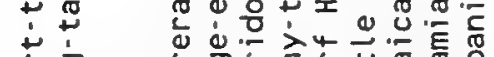

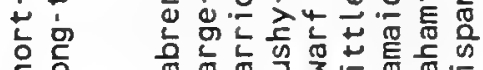

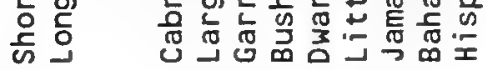

$\infty$
$c$
0
0
0
0
0

空

崖

世

$\frac{\sqrt[0]{0}}{\frac{10}{4}}$

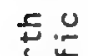

20

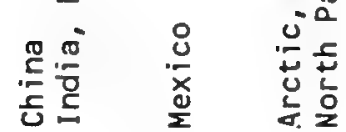

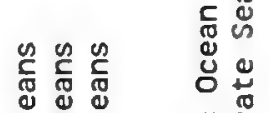
¿

まきま

蒈

ш

$w>w$

แ 


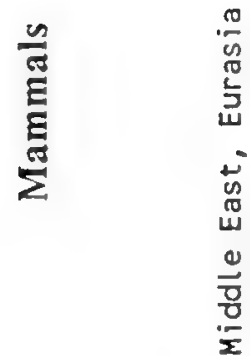

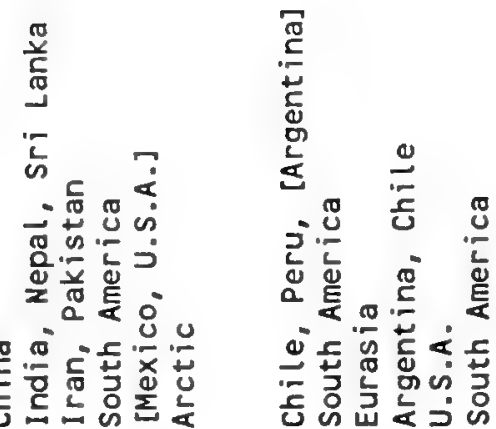

务皆

$\frac{4}{2}$

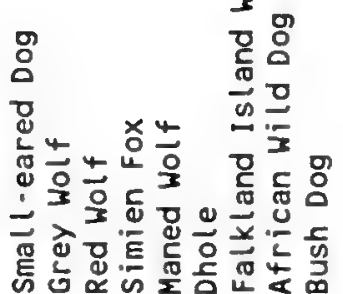

\section{要}

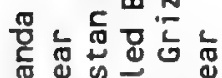

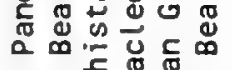

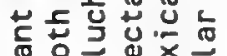

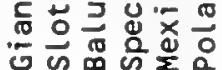

㐫岕

ปับ

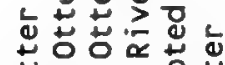

范昰

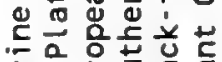

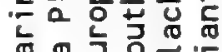

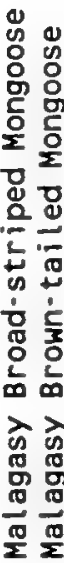
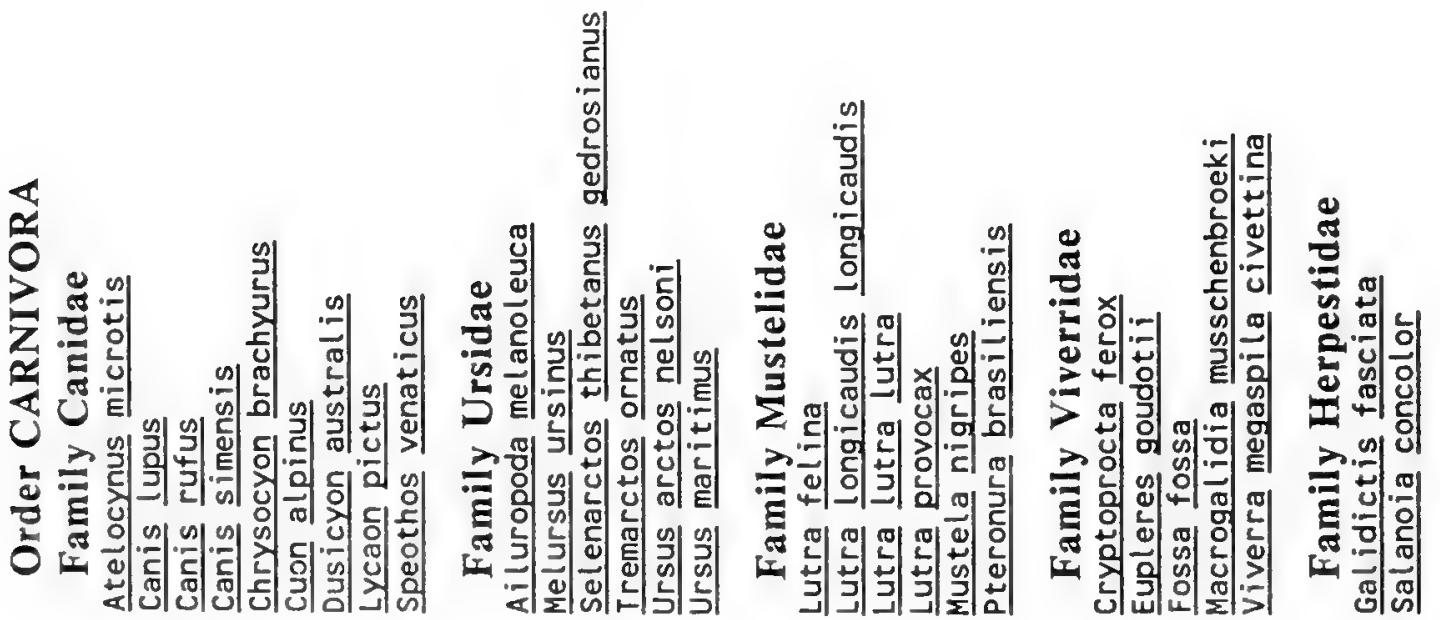


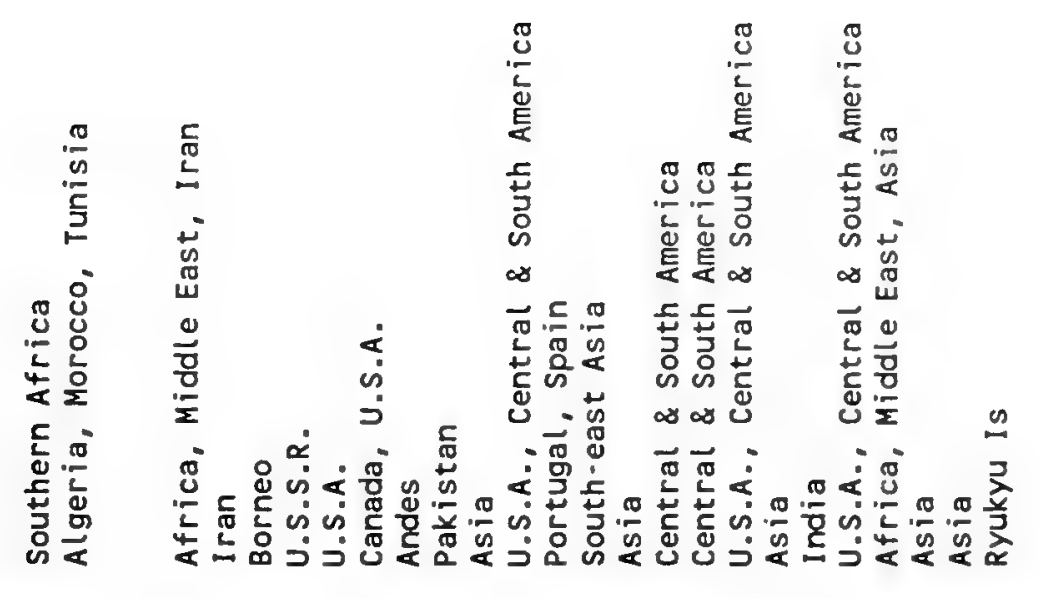

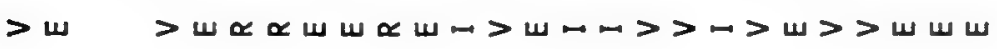
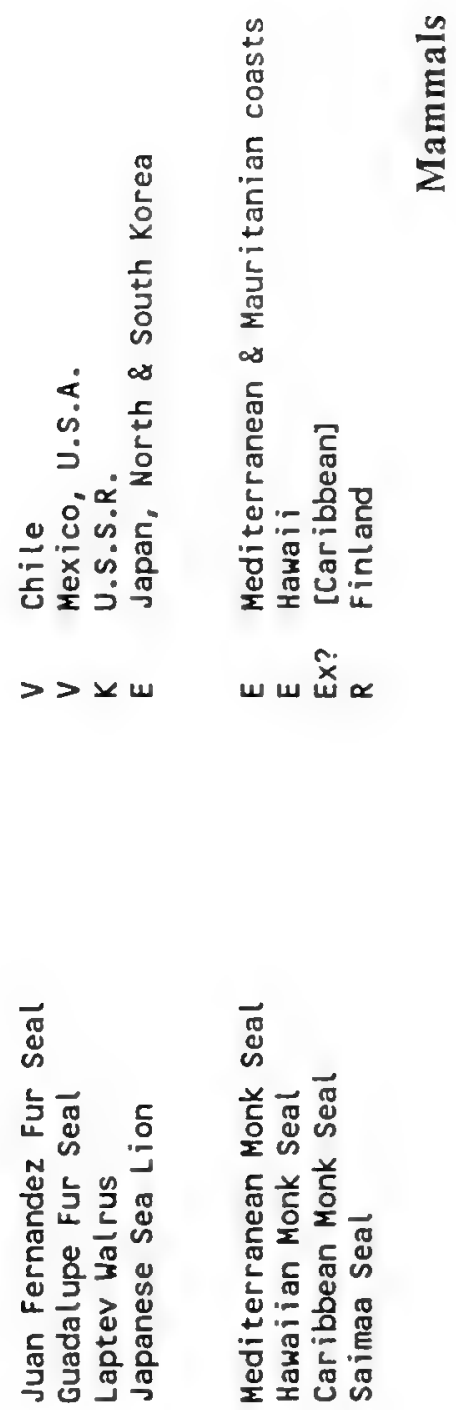

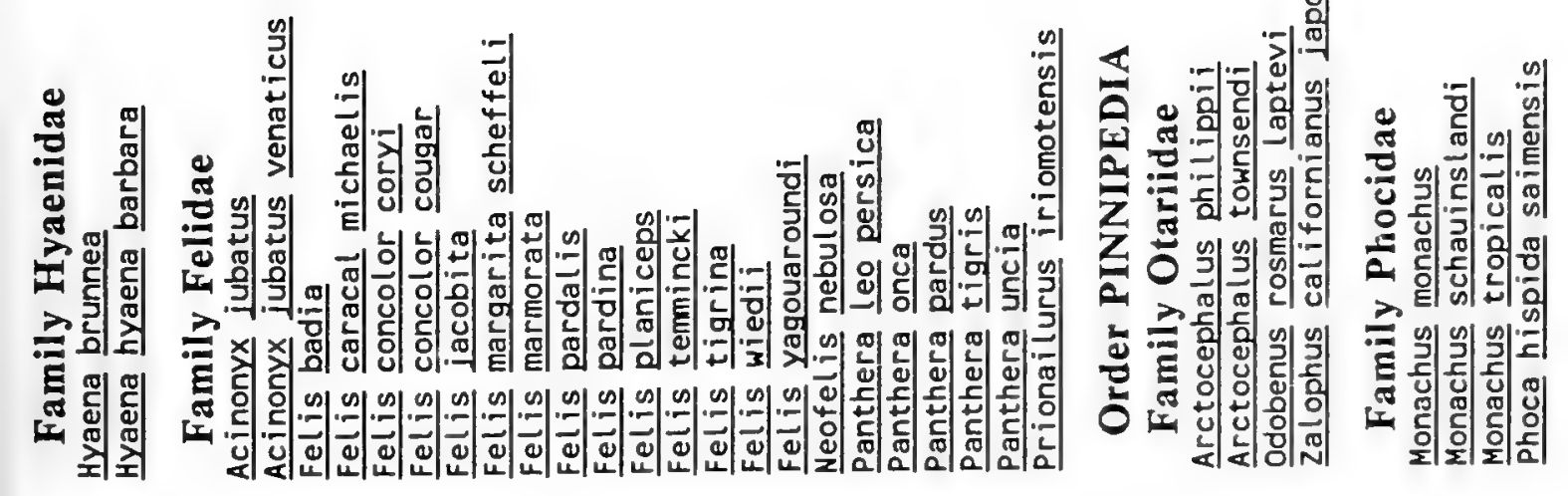


$\frac{\frac{n}{4}}{\frac{10}{2}}$

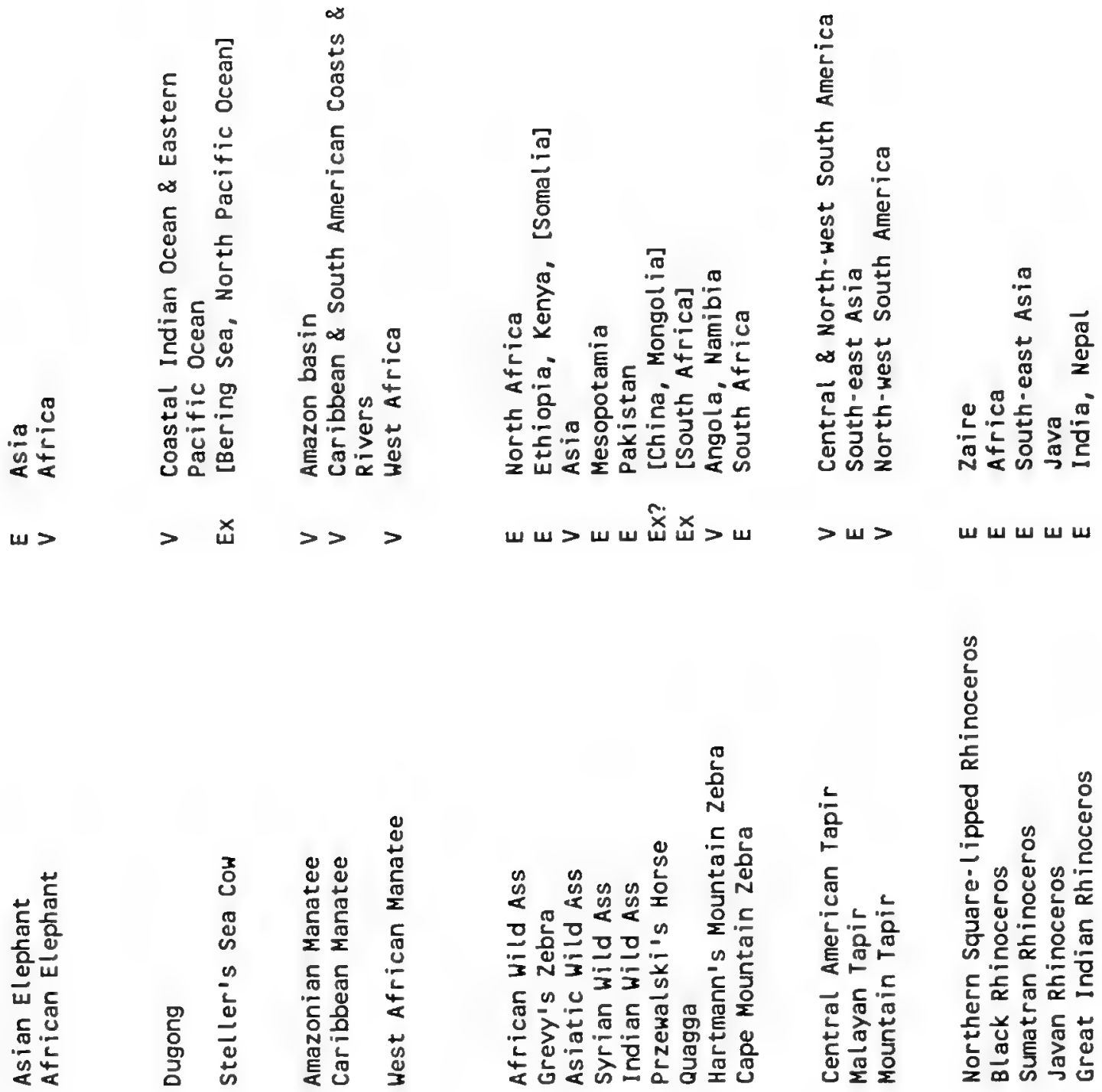
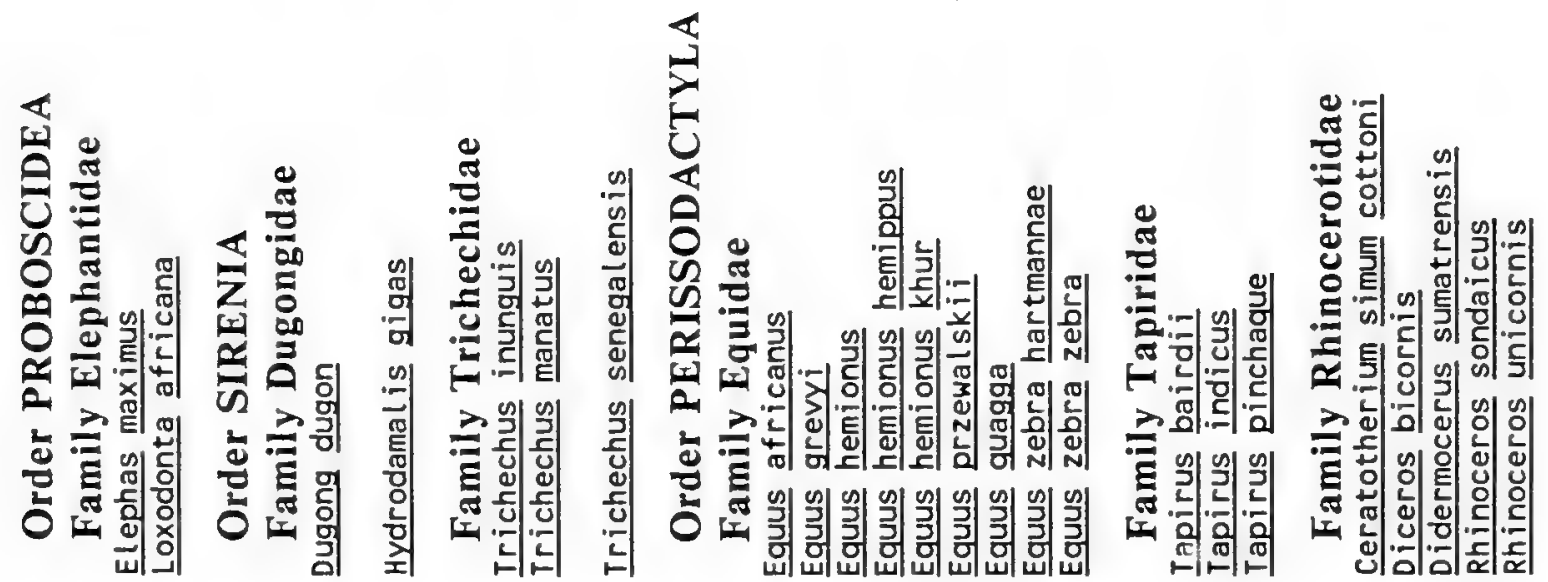

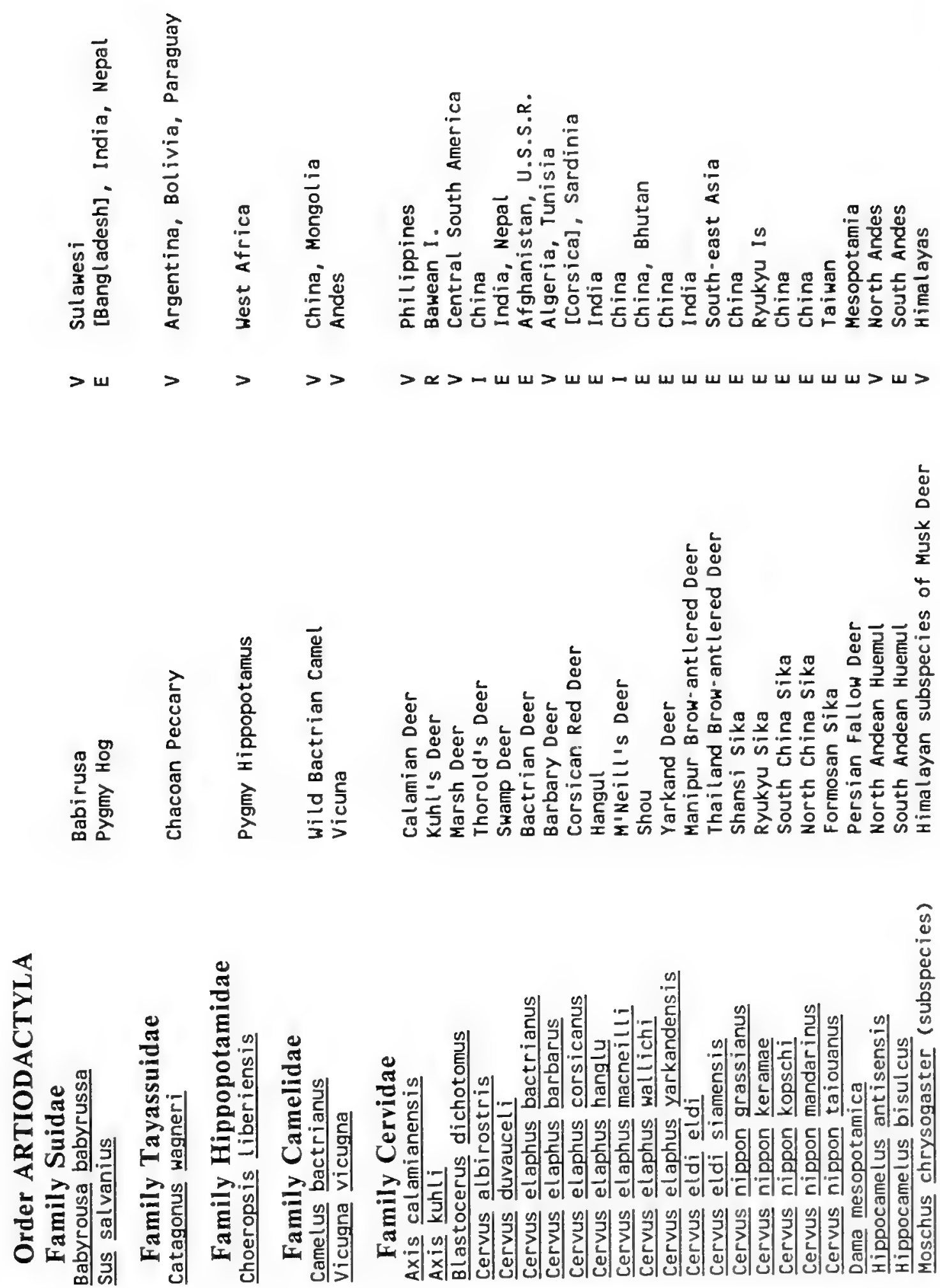
$\frac{\sqrt{5}}{\frac{5}{6}}$

ㄷำ

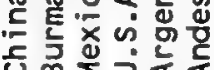

$\varpi \omega \propto \propto \omega \varpi$

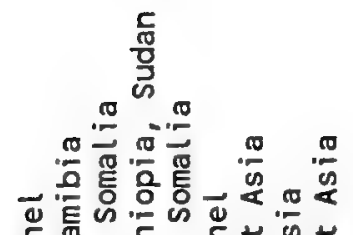

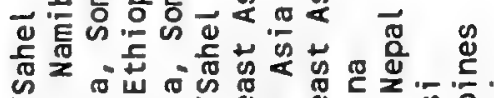

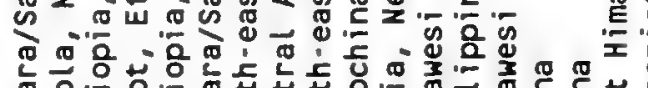
出

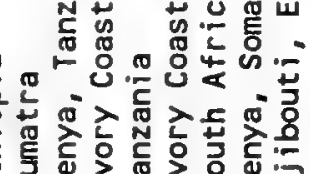

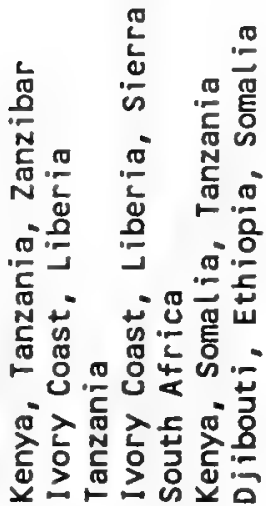

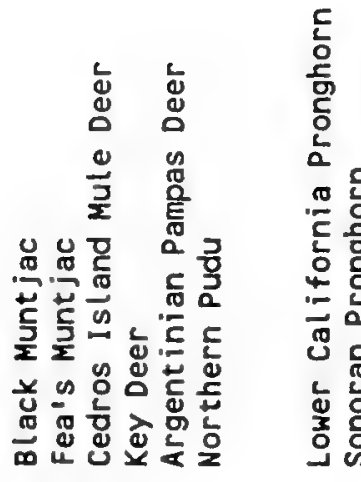

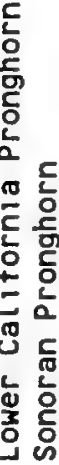

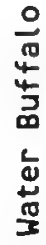

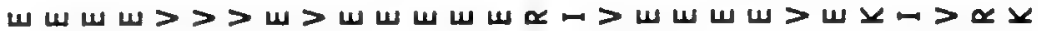

苋

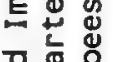

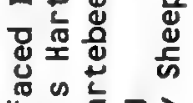

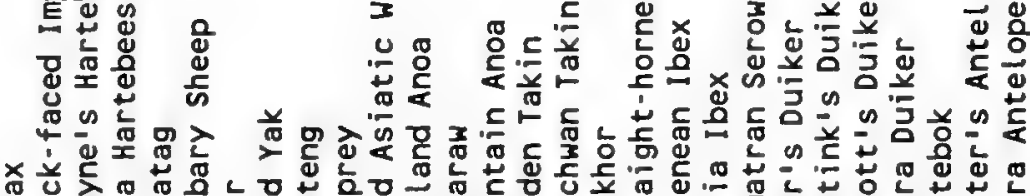

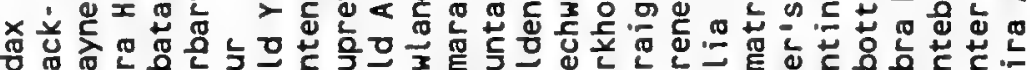

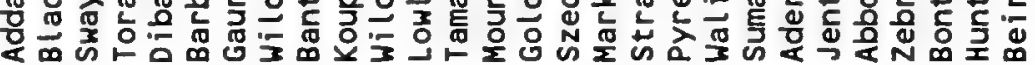
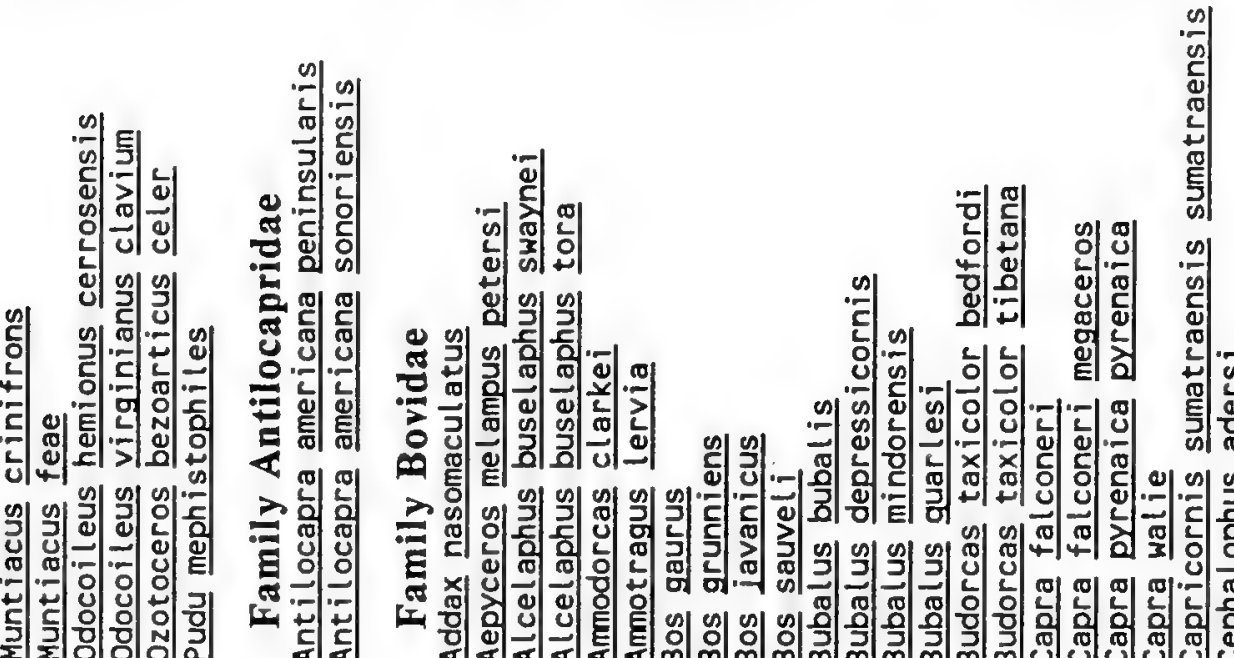

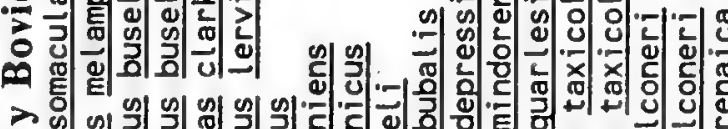

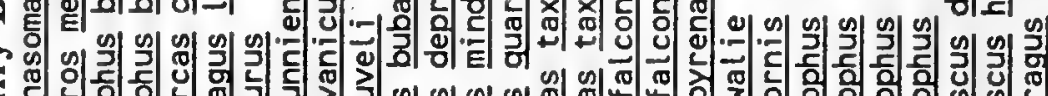

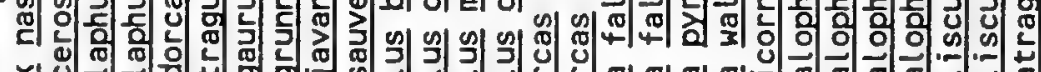

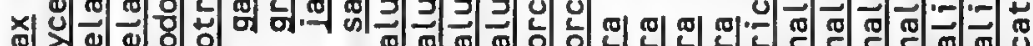

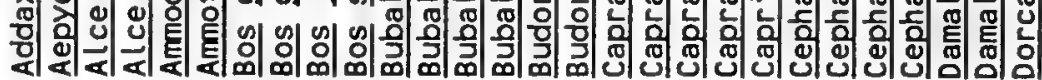



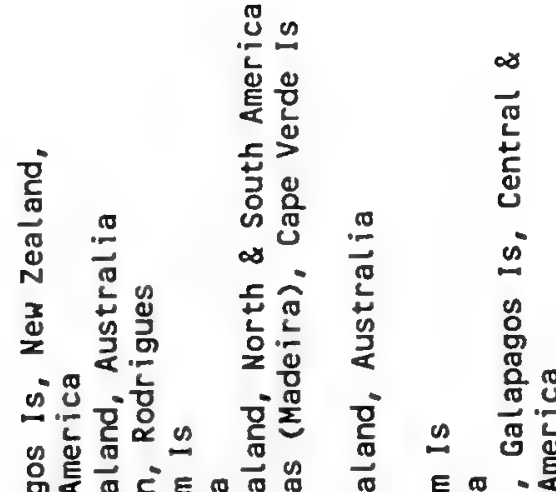

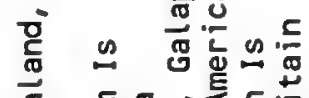

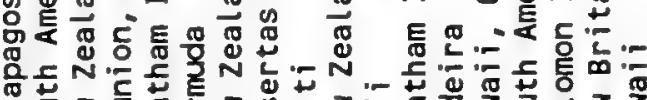

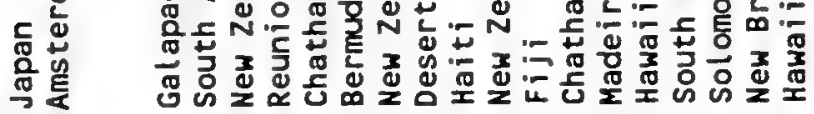

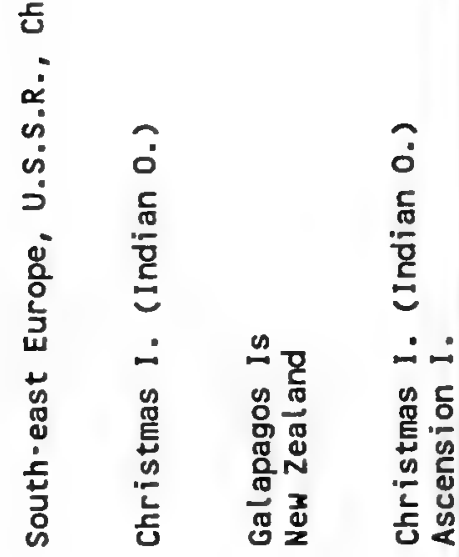

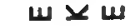

แ

ш

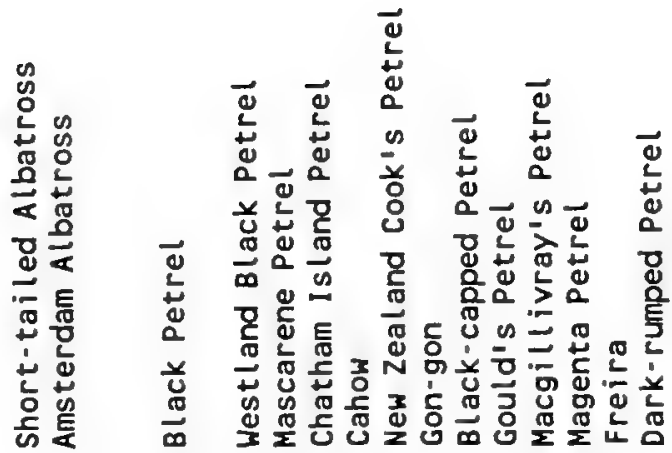

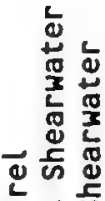

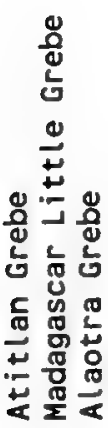

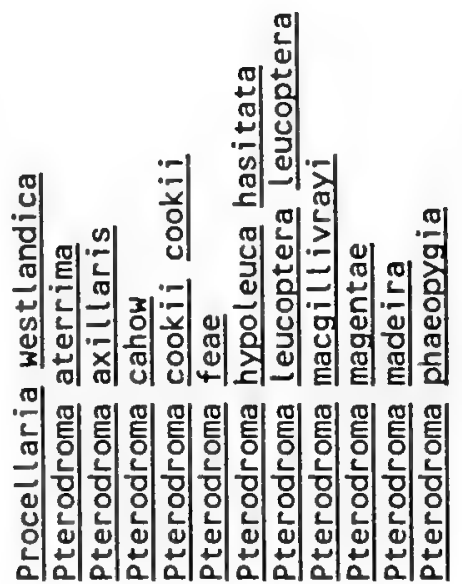

의 क्ञा
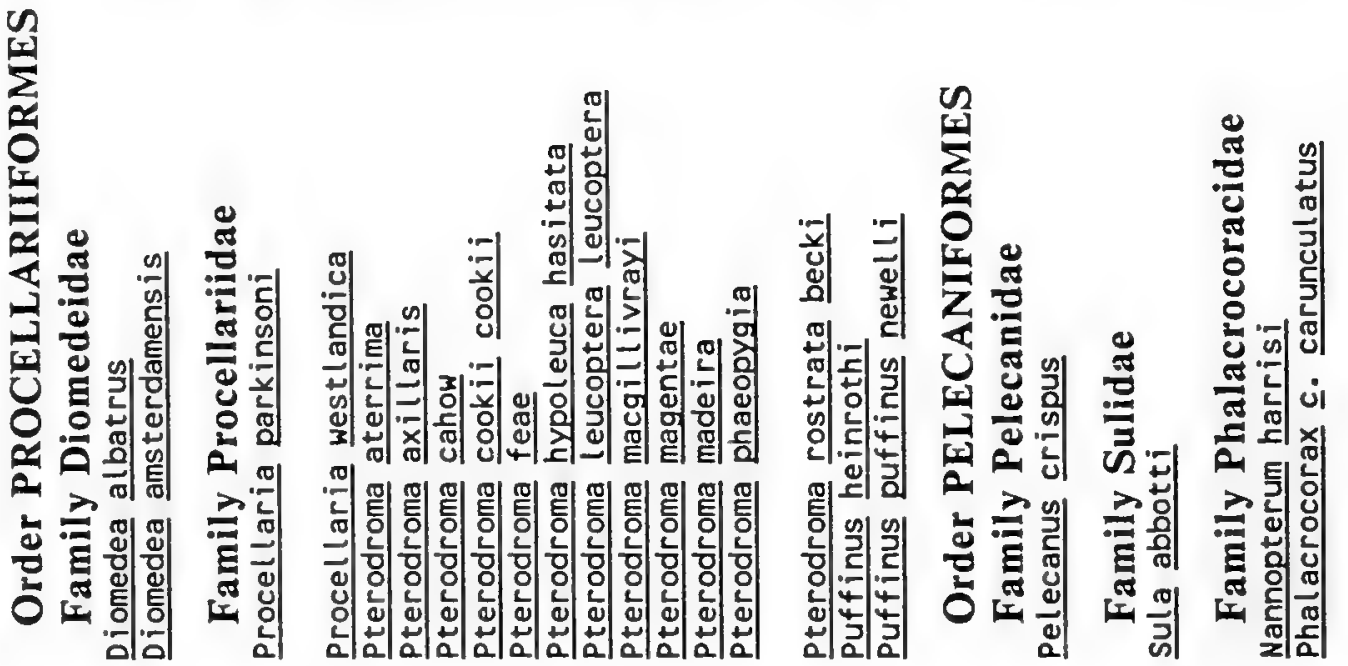

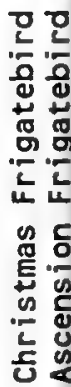
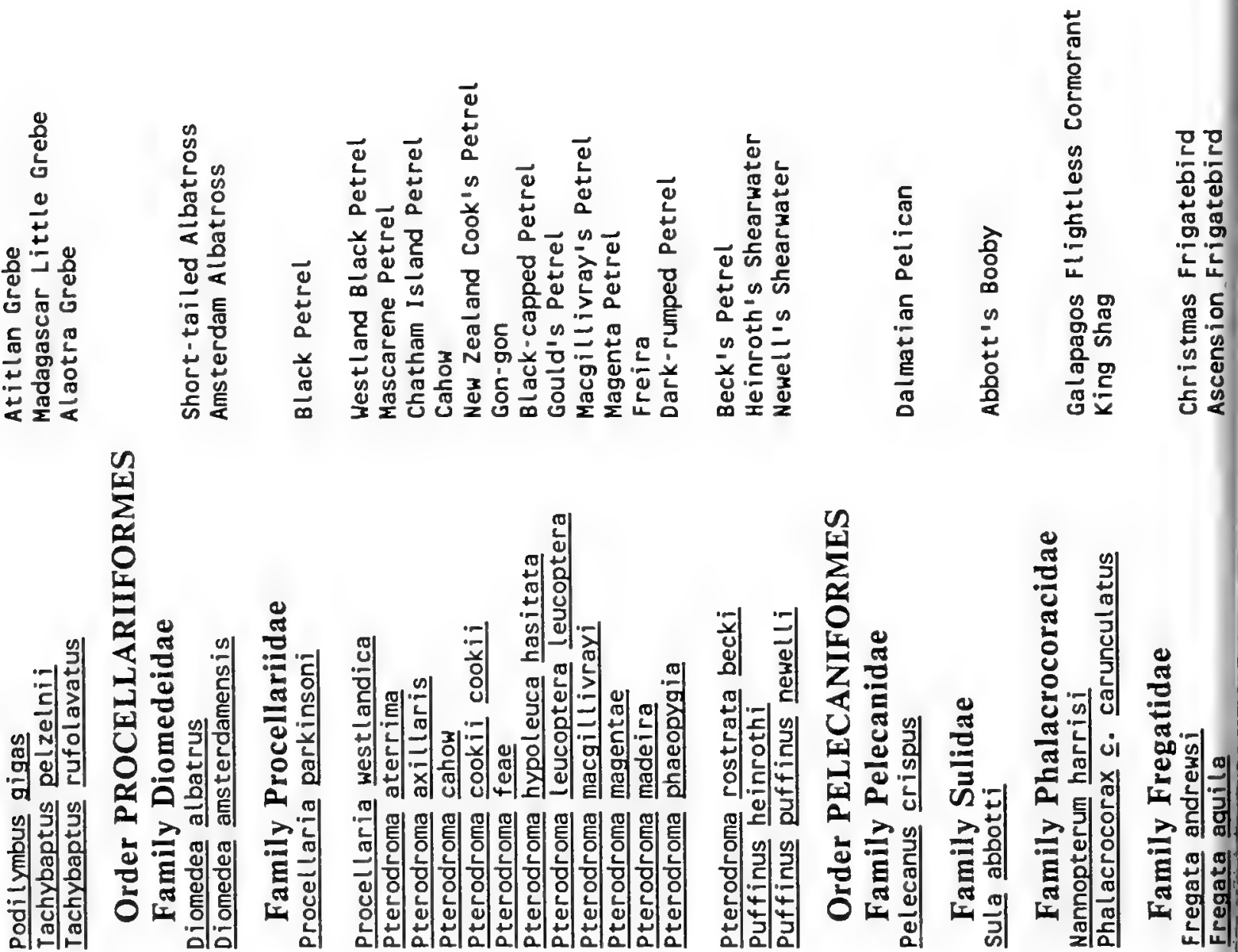
$2 \pi \frac{\pi}{2}$

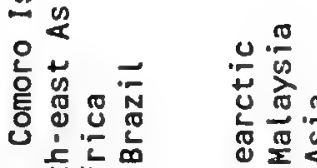

兵安

बรष

距 $ᄃ$.

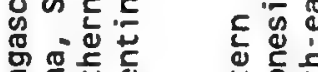

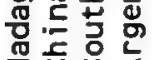

它政

总总范
는

点

$\stackrel{0}{0}$

$\sum \quad \frac{0}{2}$

웡 एँ

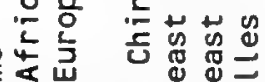
둥됴

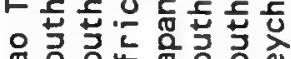

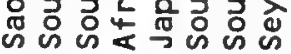

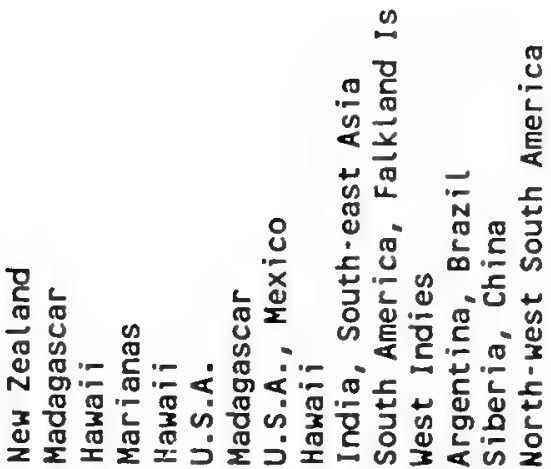

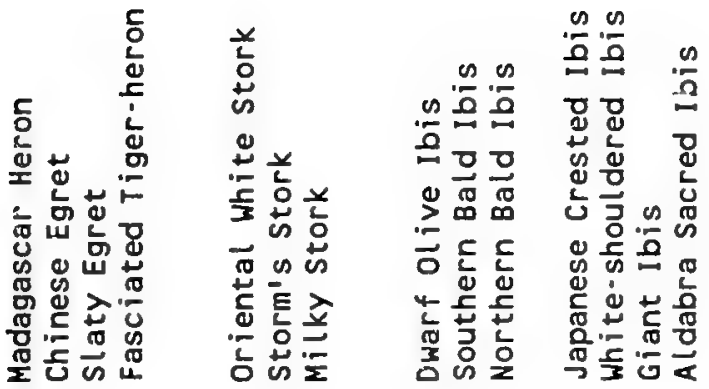
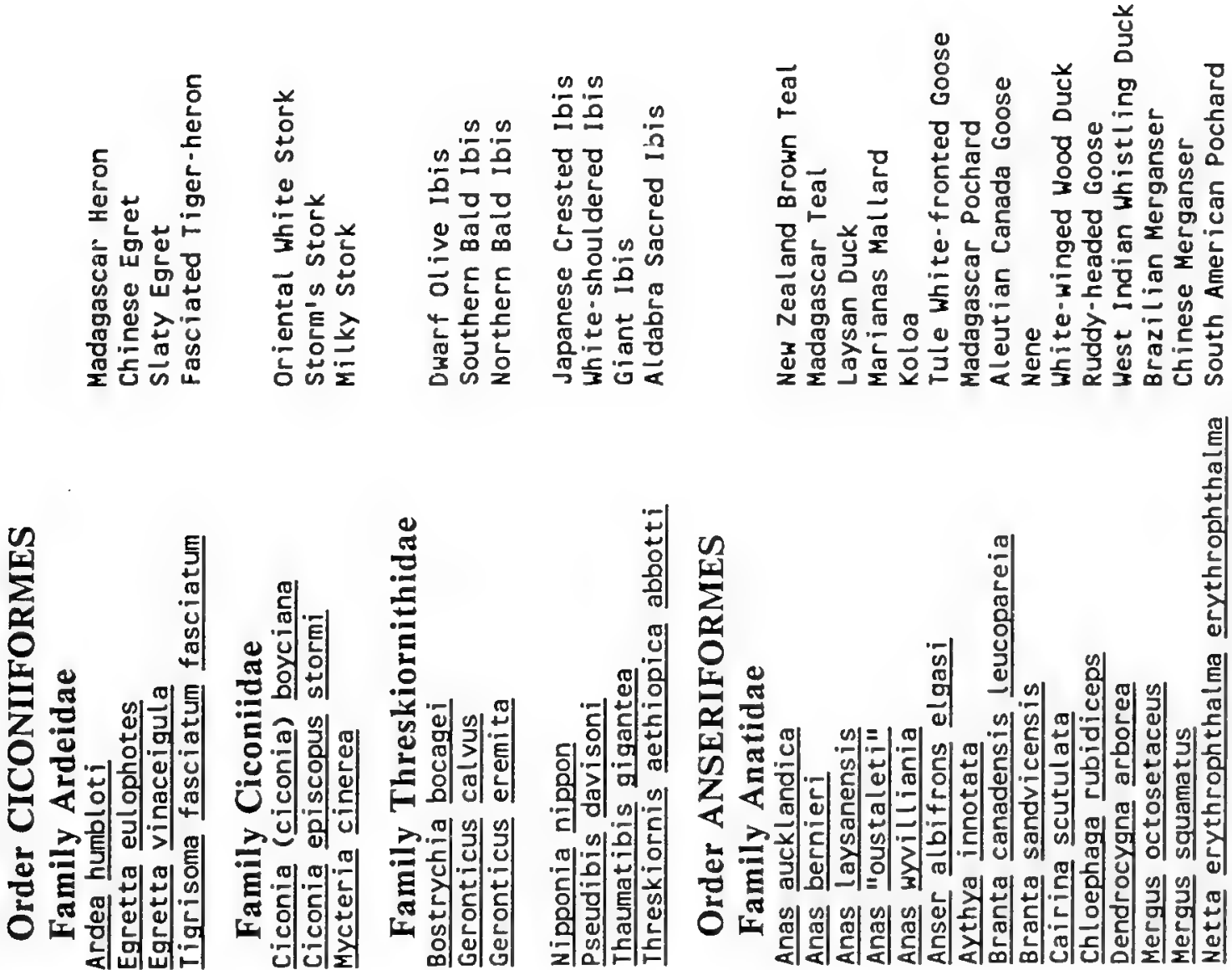

点
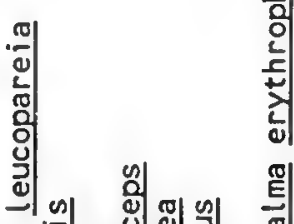

$\stackrel{2}{\tilde{\pi}}$

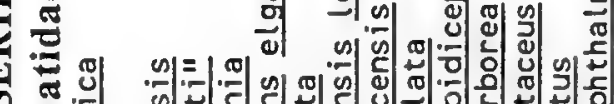

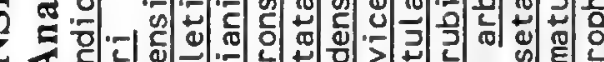
4
0

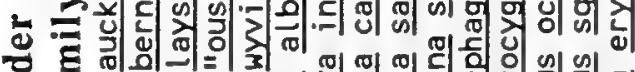
인

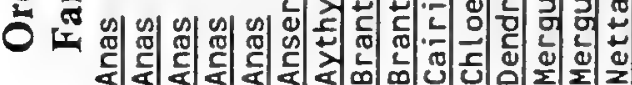




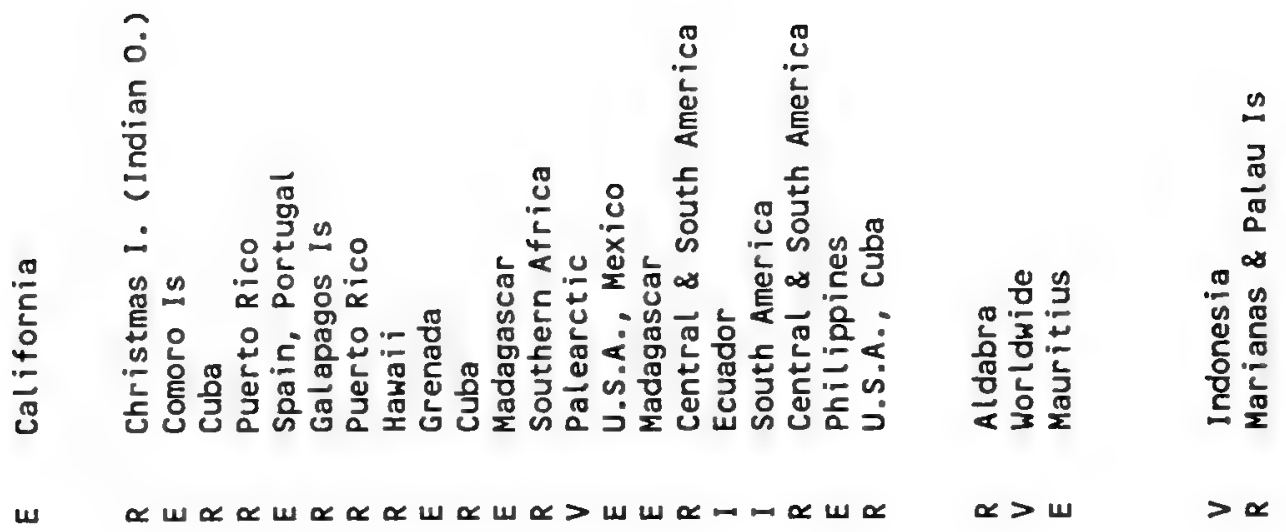

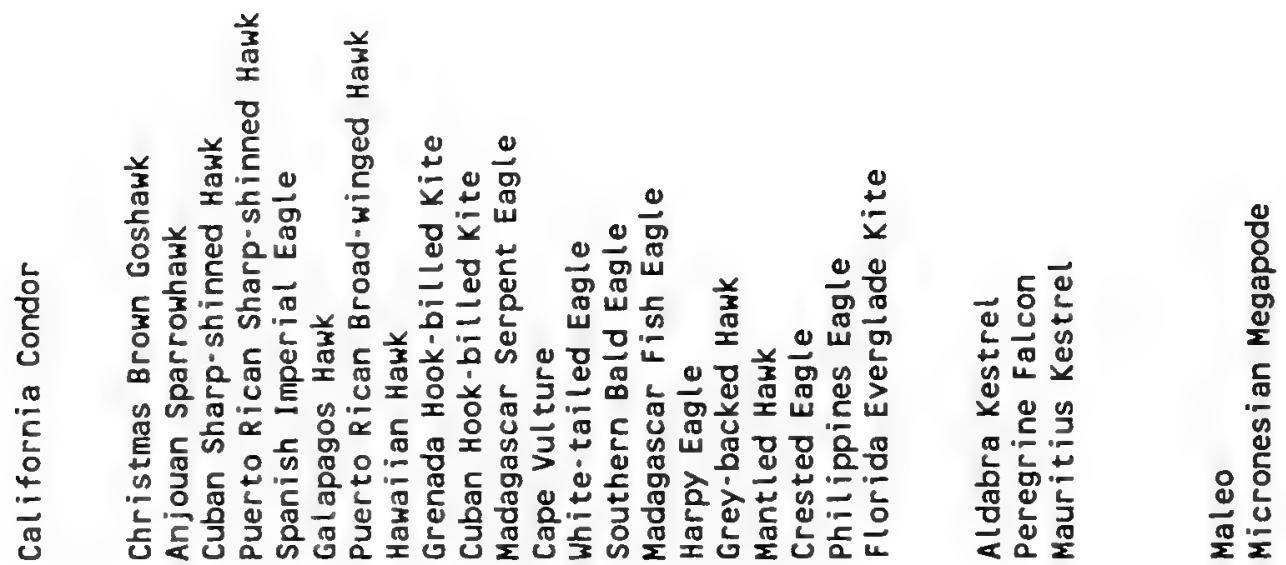

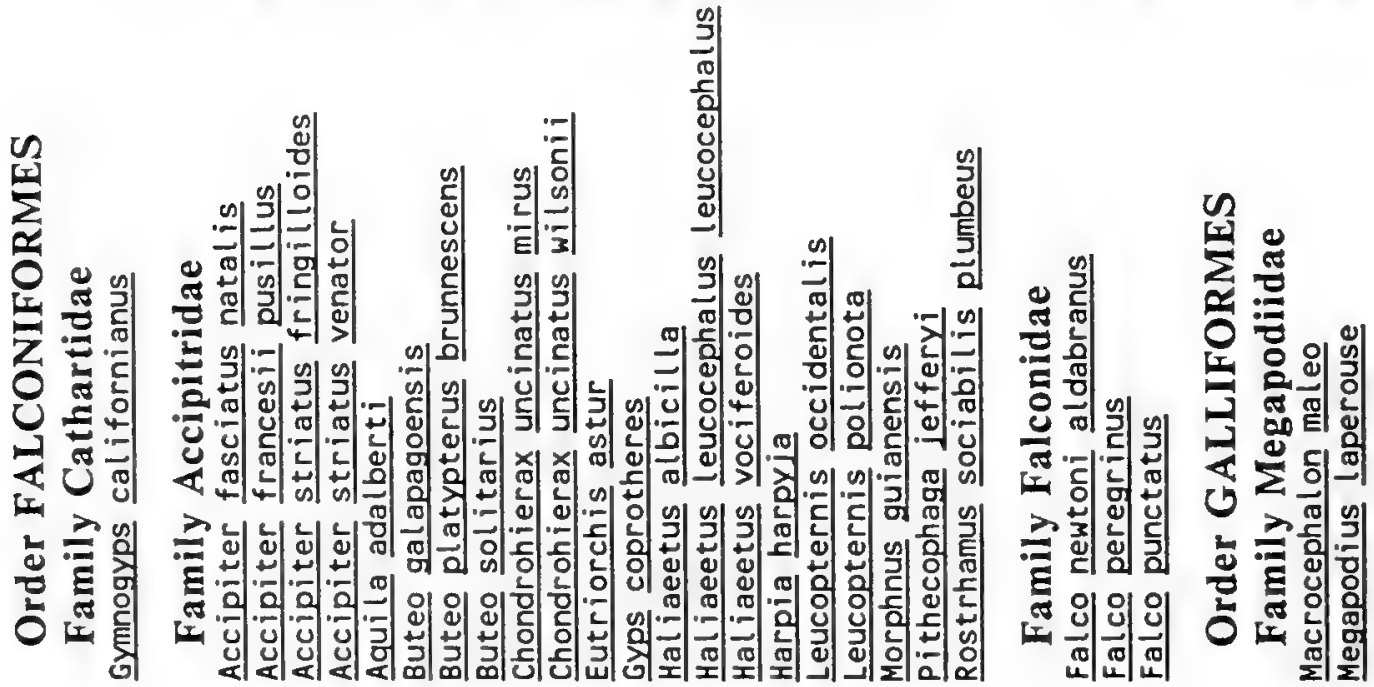




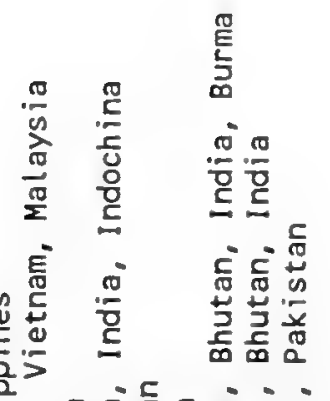

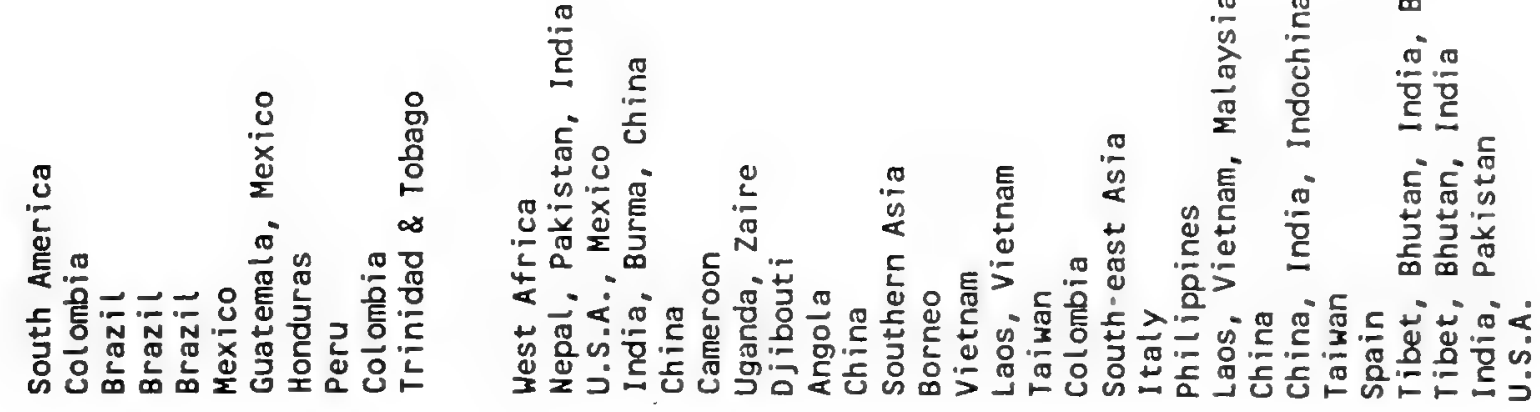

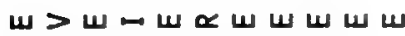

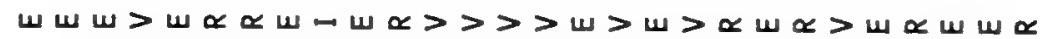

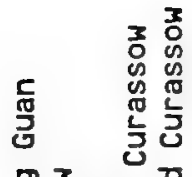

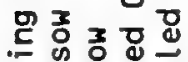

을

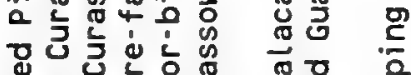

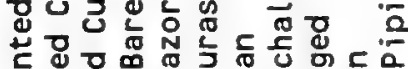

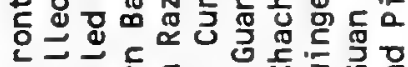

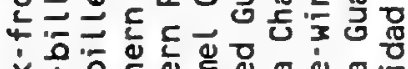

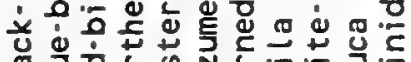

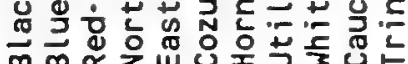

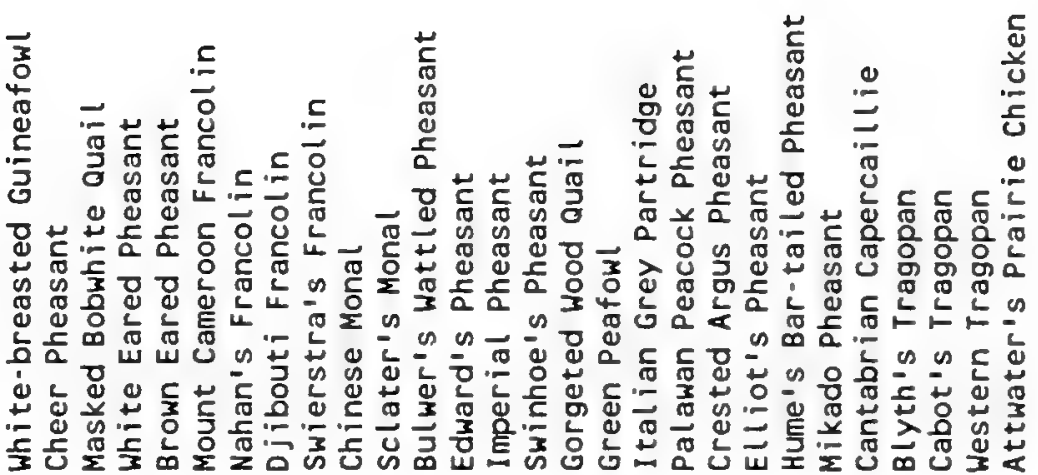

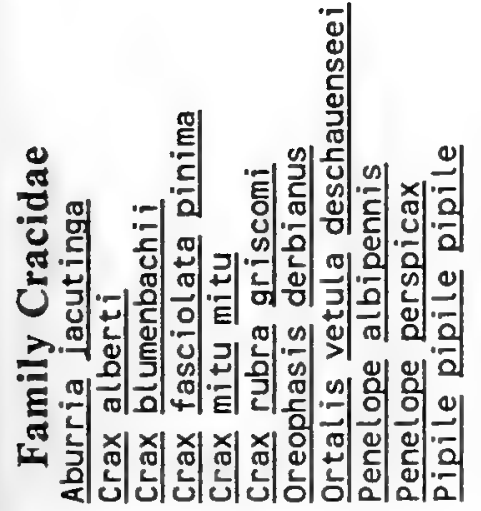

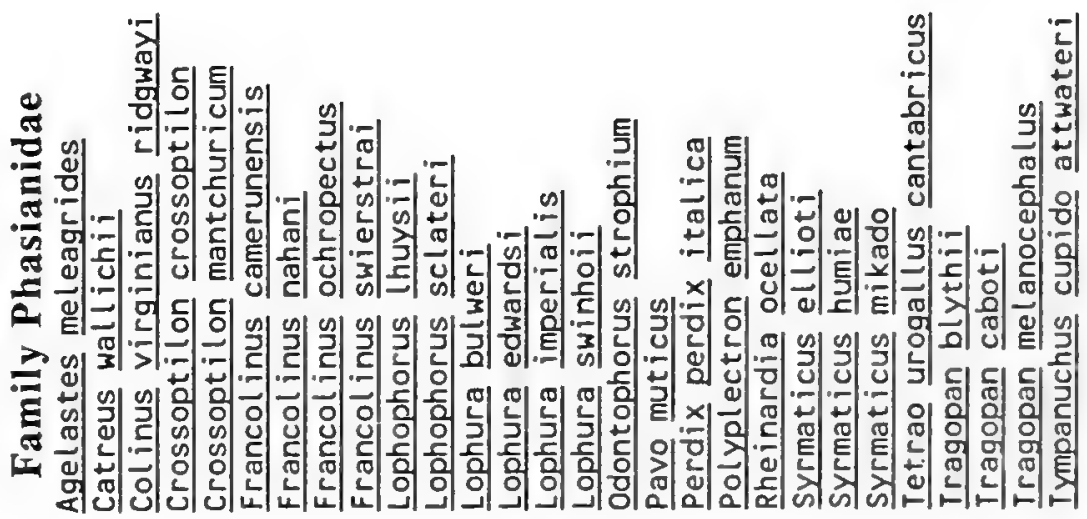


党

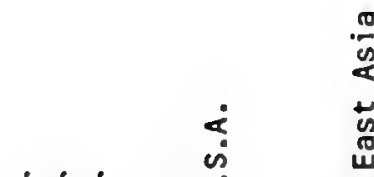

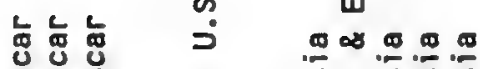

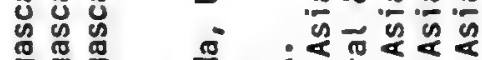
(⿻)

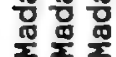
ช $\pi<\omega$

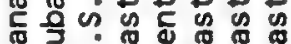

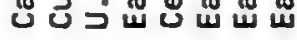

soc

$$
\stackrel{0}{\frac{5}{5}}
$$

$\frac{8}{4}$

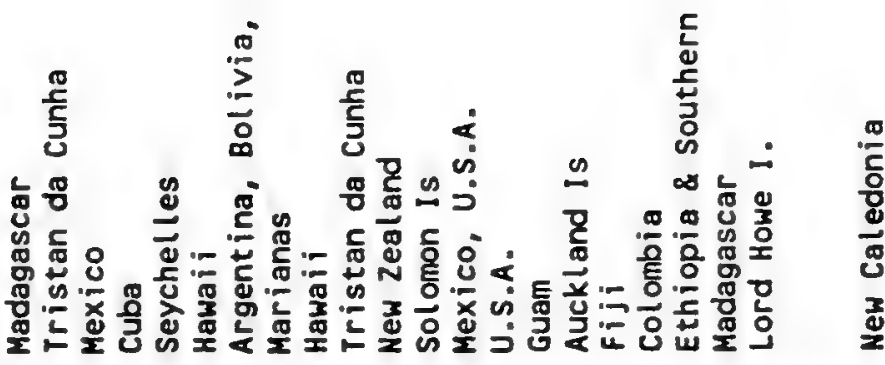

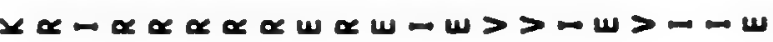

w
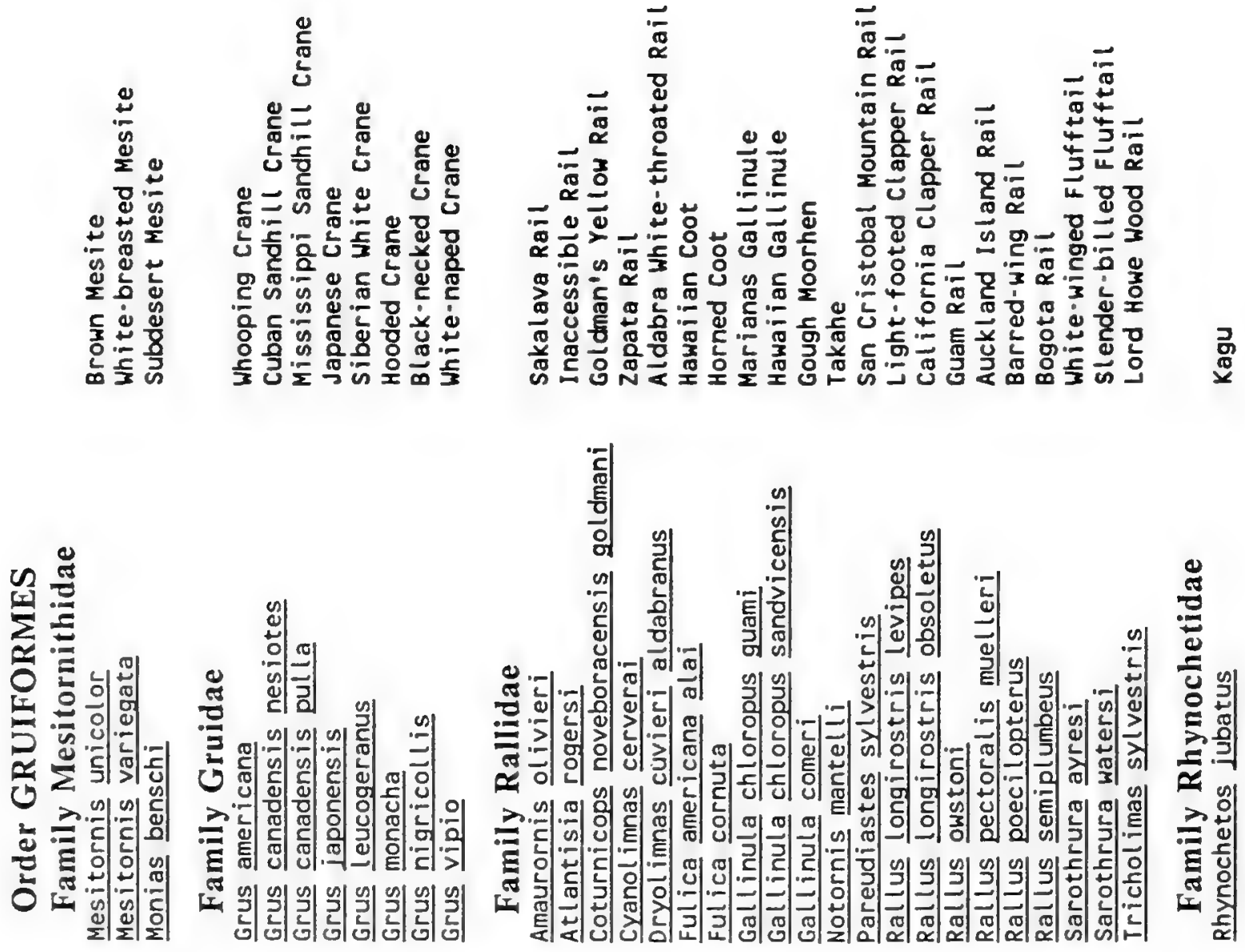

+ \&

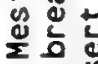

织

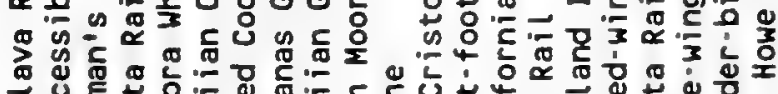

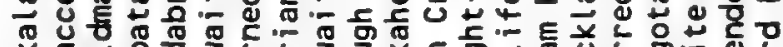

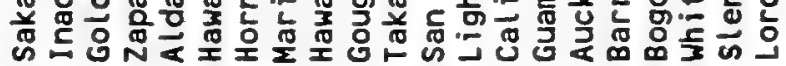

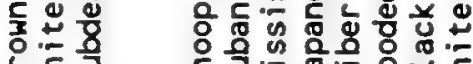

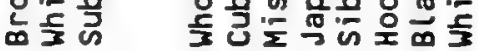


ᄂ⿺辶

范敂

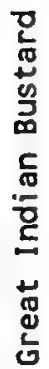

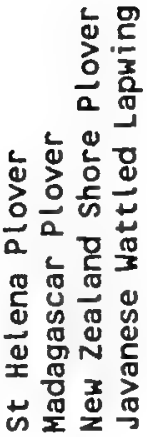

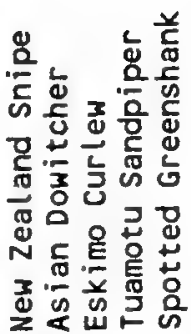

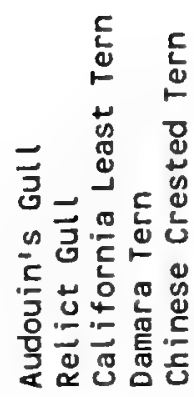

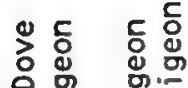
응

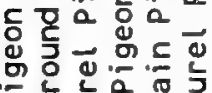

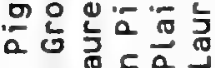

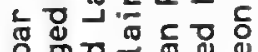

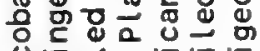
중 ว

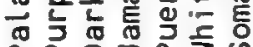

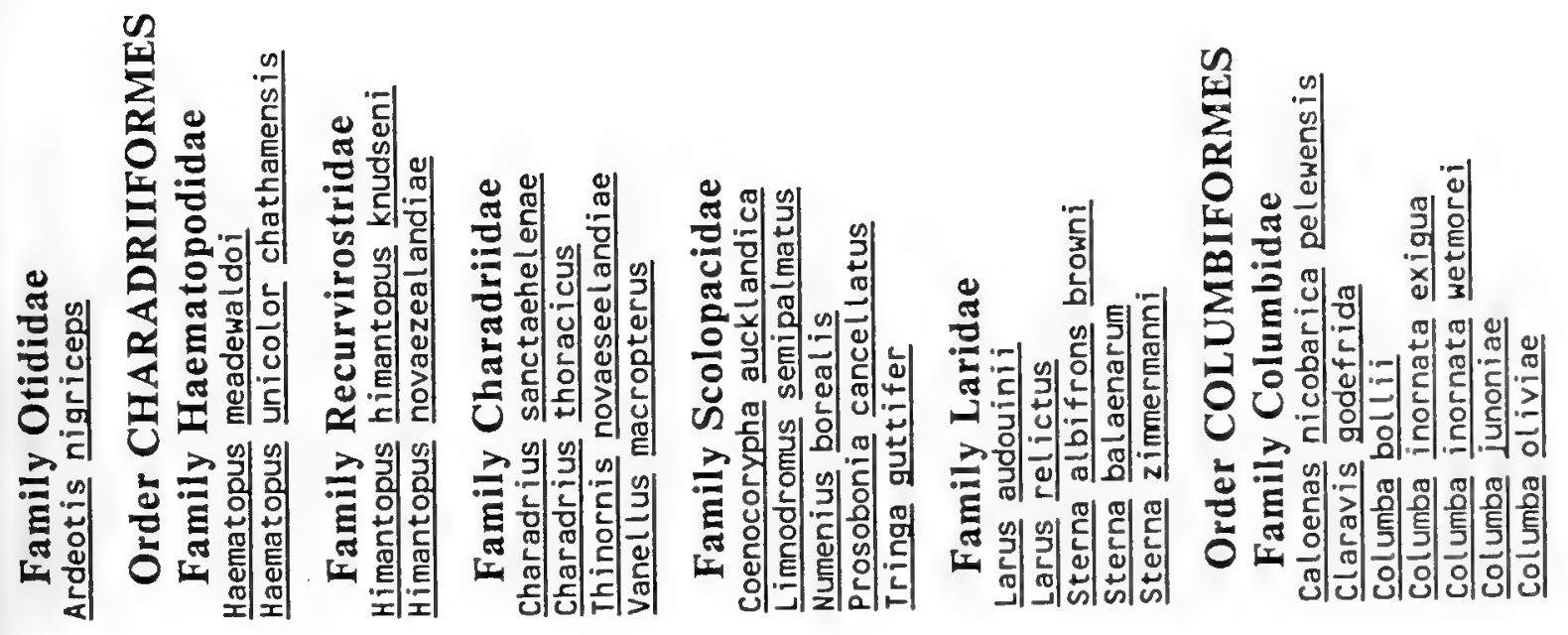



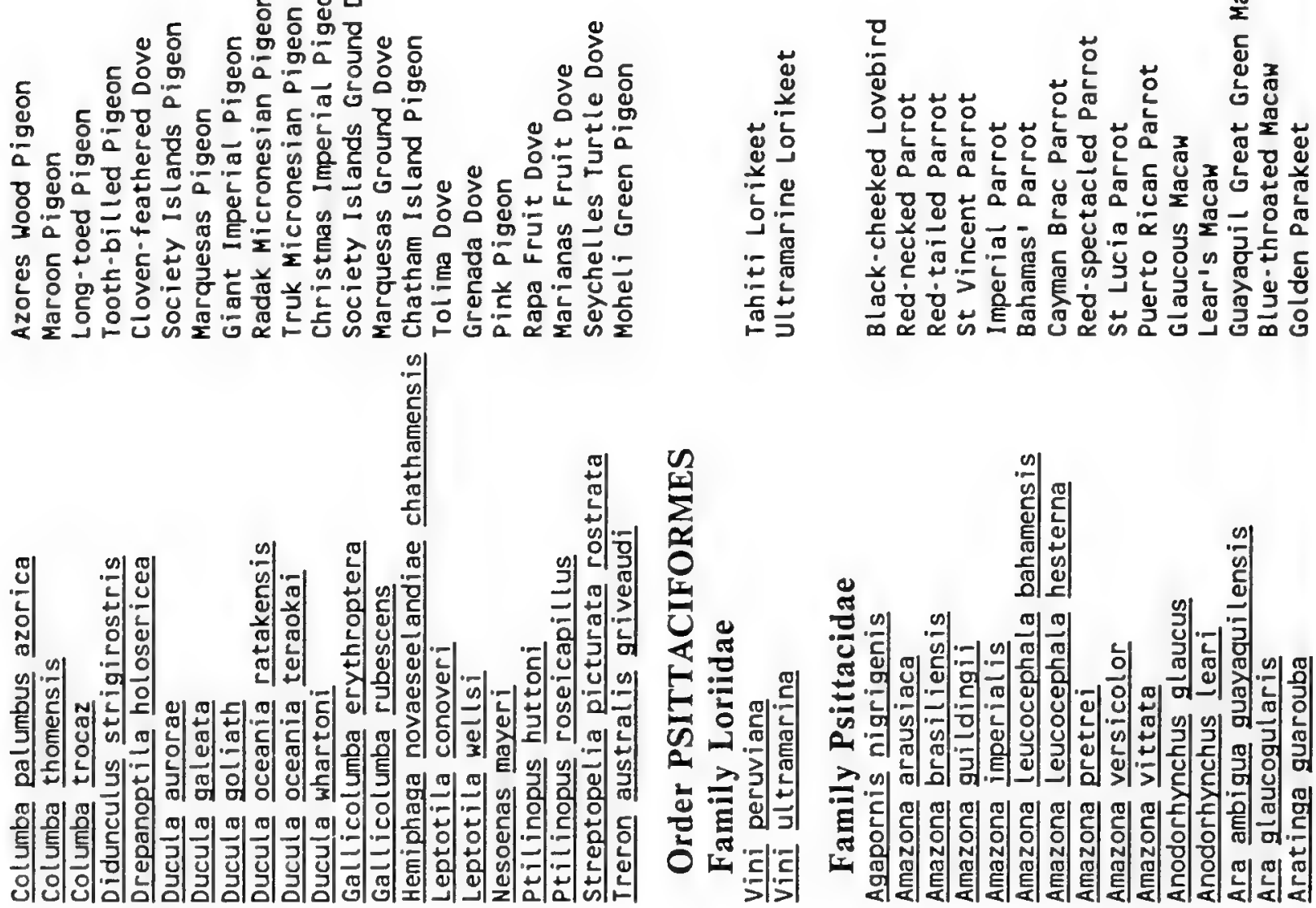


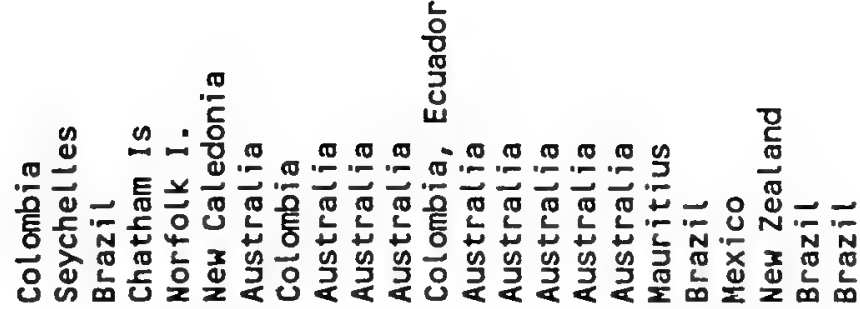

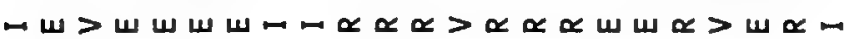

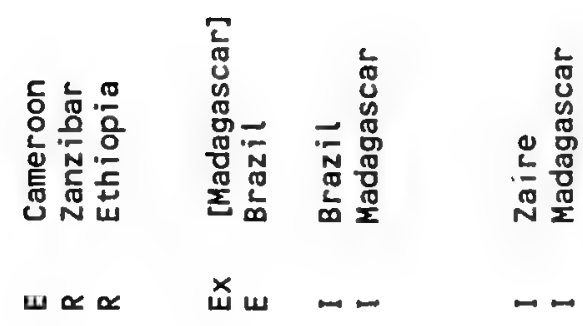

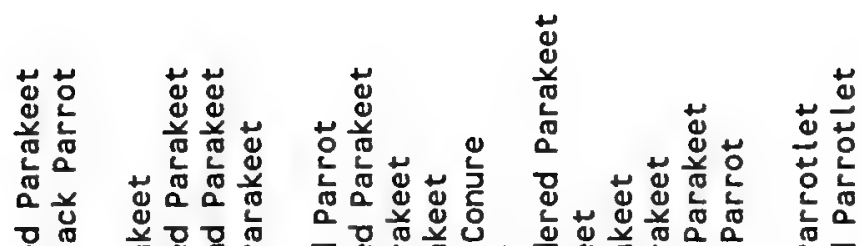

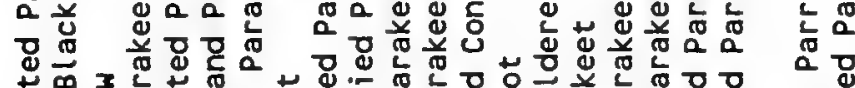

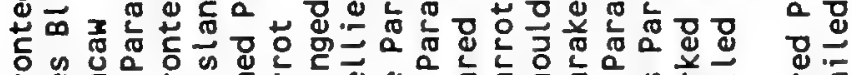

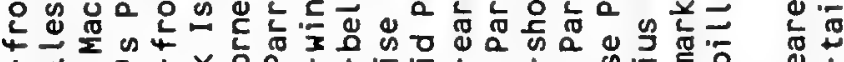

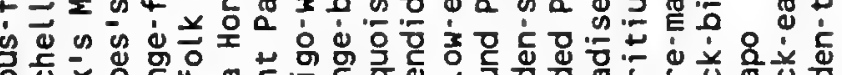
돈

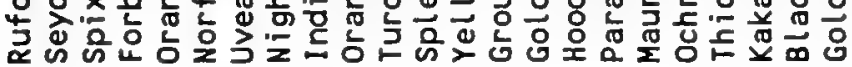
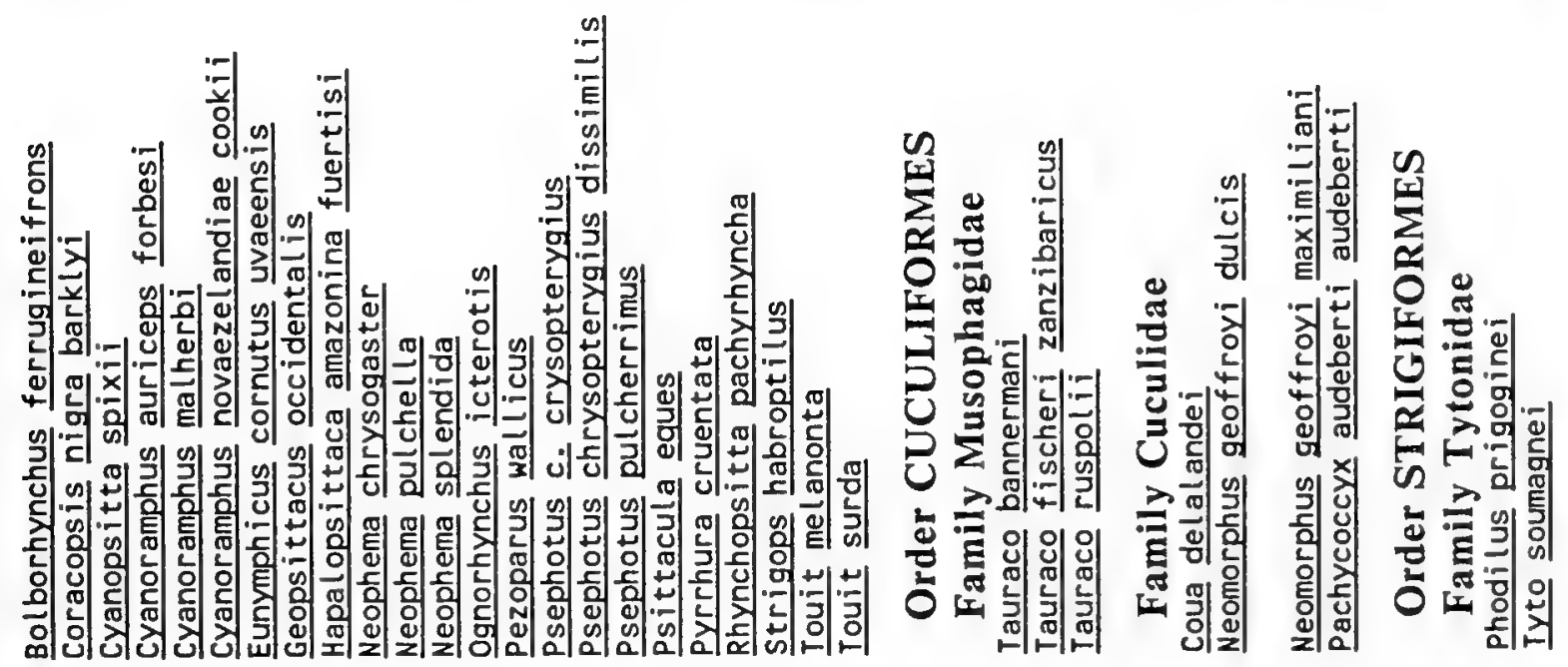

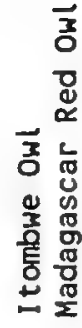




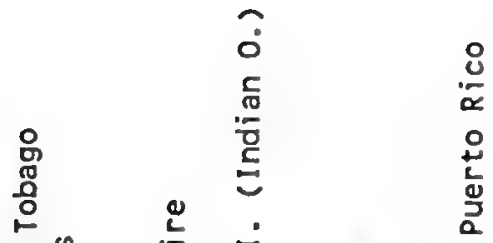

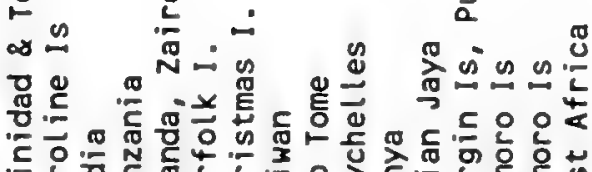

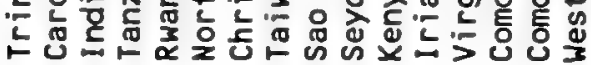

$\propto \alpha-\propto \alpha-\alpha w \propto \alpha \omega-\alpha \propto U \propto$

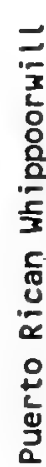

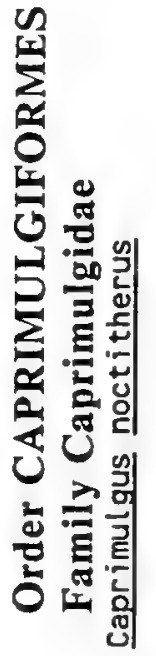

$\frac{\pi}{\stackrel{0}{0}}$

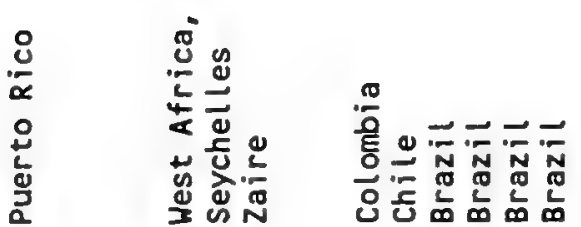

ك.

苞

$\propto$

$x \propto-$

-

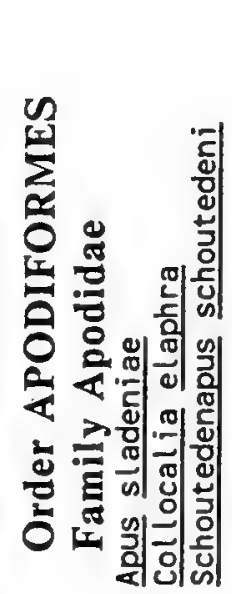

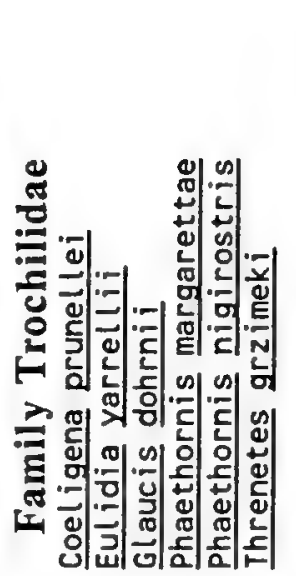

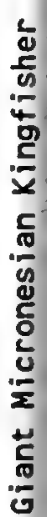
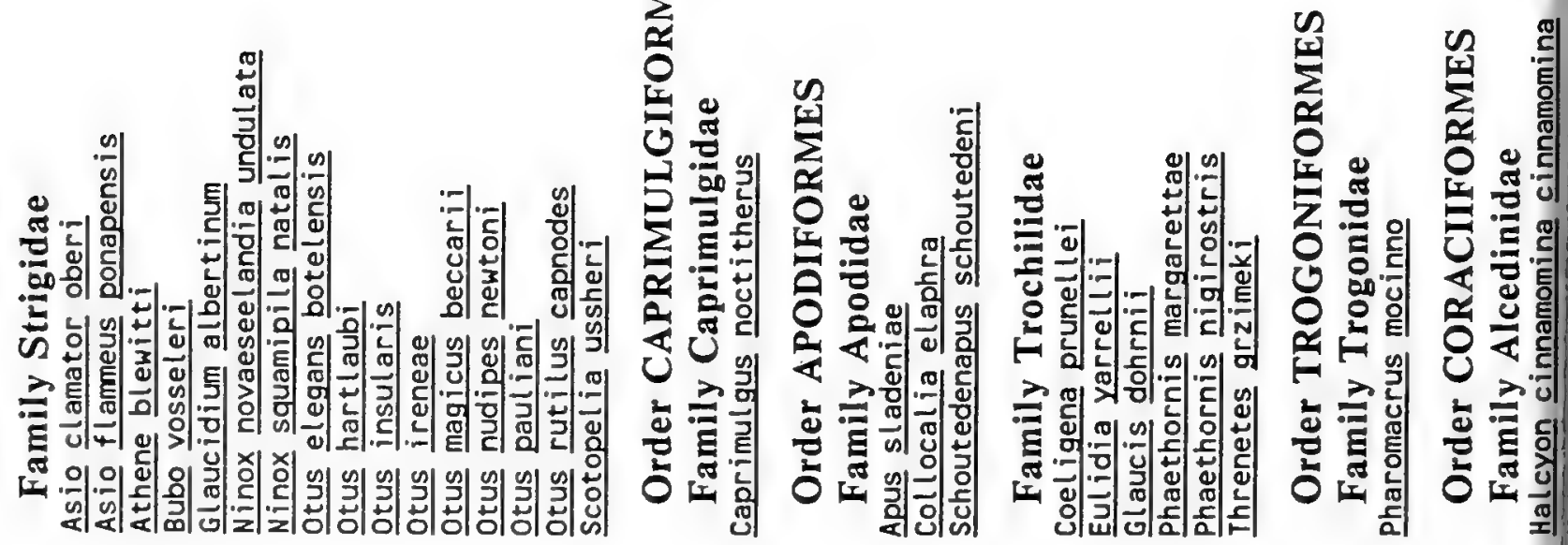

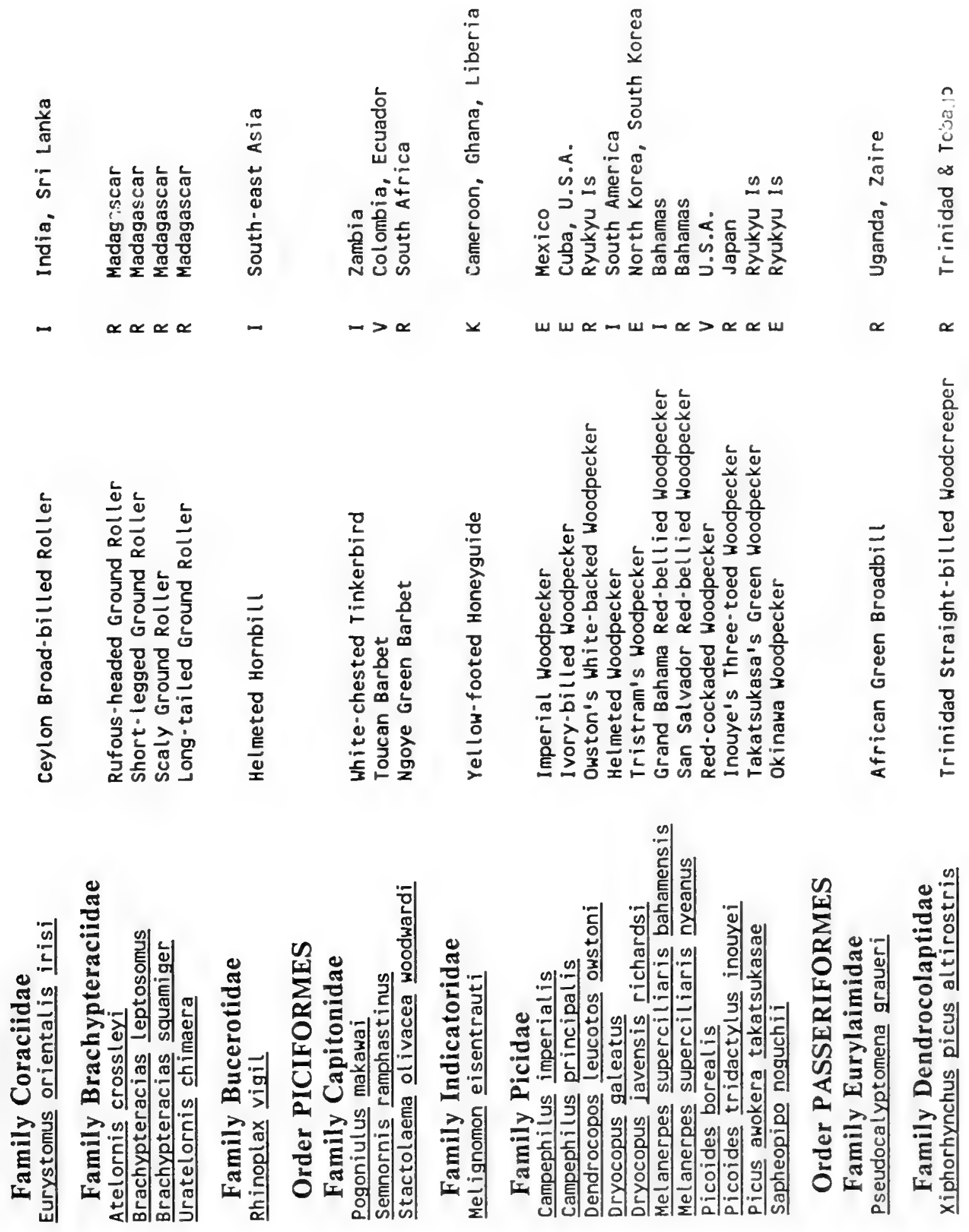
<smiles>[CH]C=[Os]</smiles>

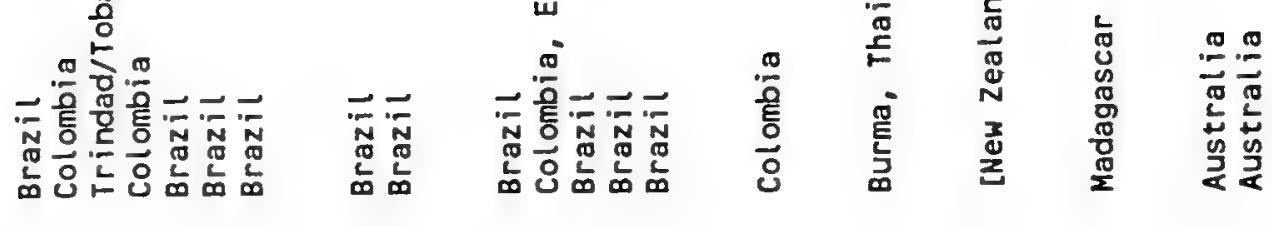

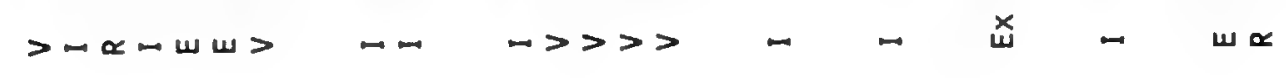

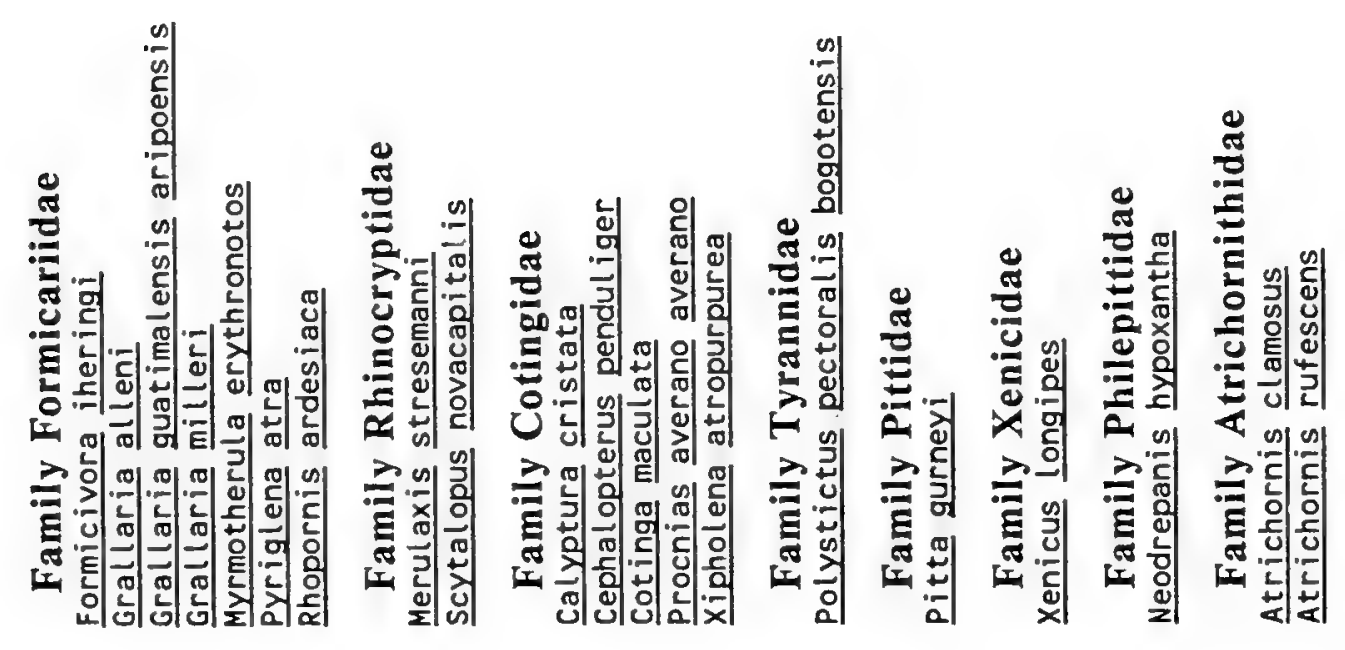


के

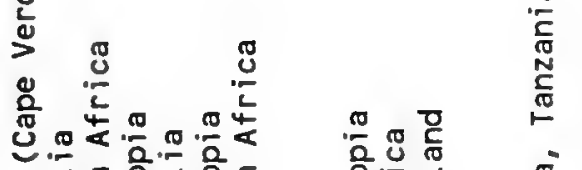

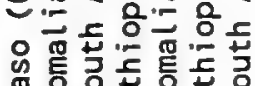

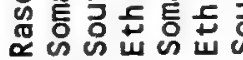

U $-\backsim \leq x \mapsto$

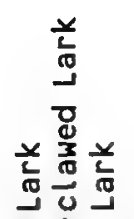

원망

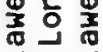

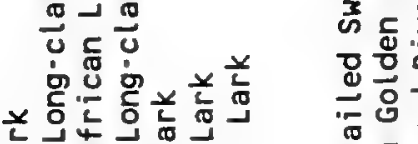

s.

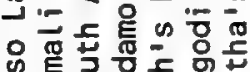

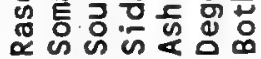

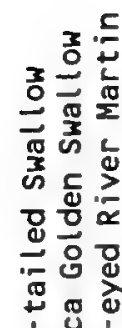

is

兵毞兵

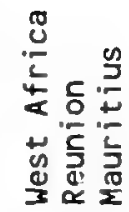

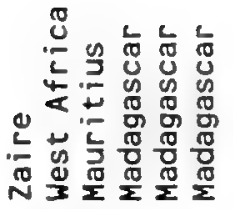

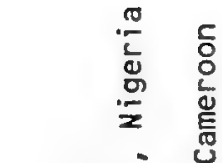

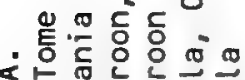
๔ト ⿱ㅗㄴ

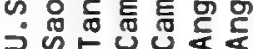

$\omega \pitchfork \propto \propto ⿻ m$ $\frac{0}{\frac{1}{2}}$

$\dot{0} \stackrel{y}{0}$

喜道旁

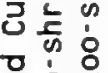

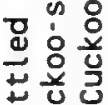

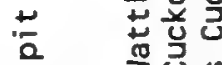

里

든 등

过它
롱

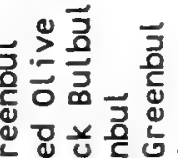

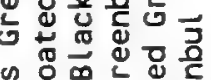

๘

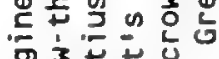

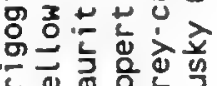

$\stackrel{2}{\frac{1}{2}}$

岳

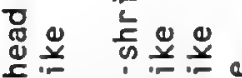

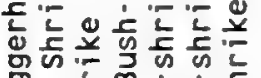

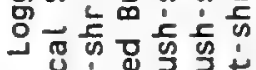

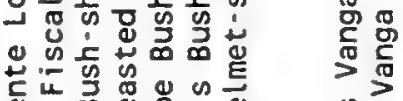

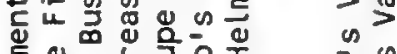
崖出

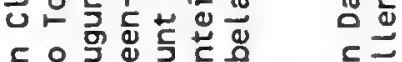

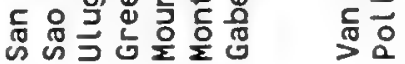

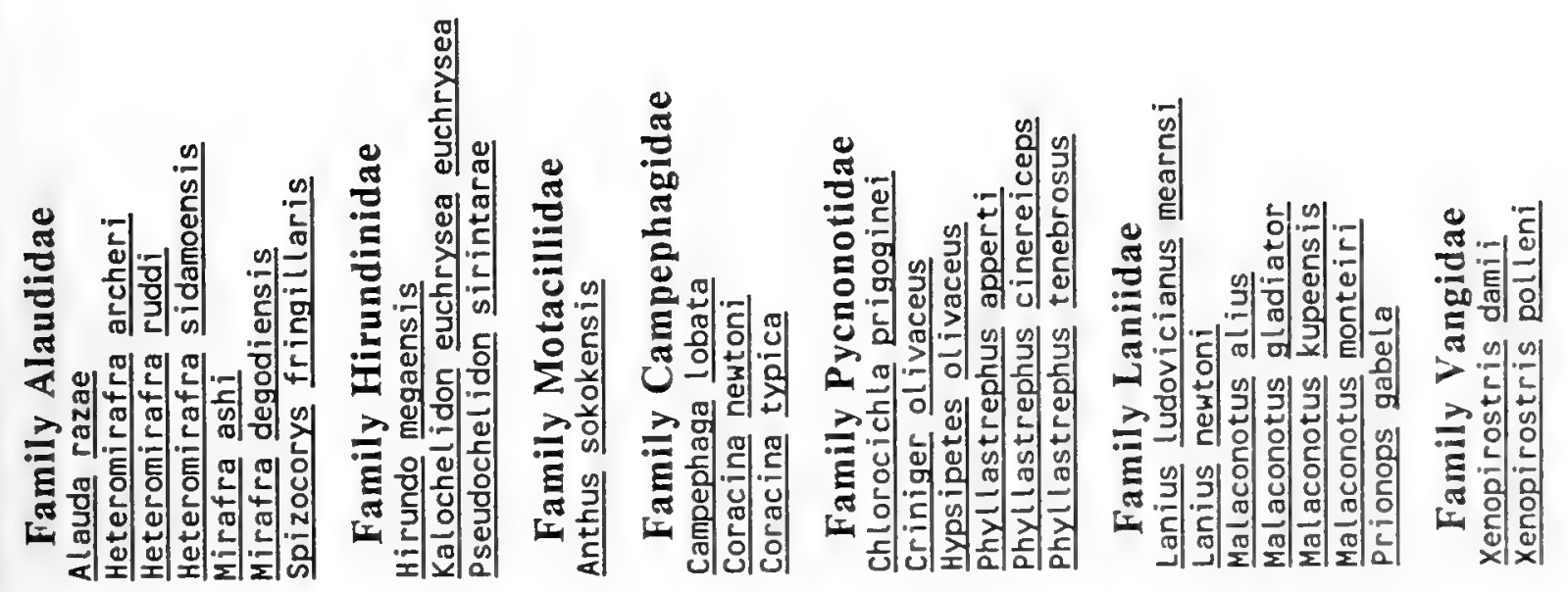




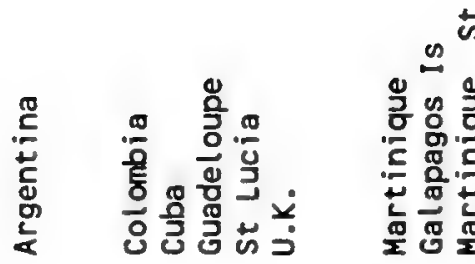

$-\quad>\propto \omega \omega \propto$

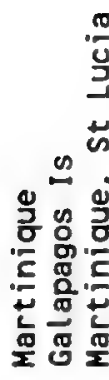

u $\propto$

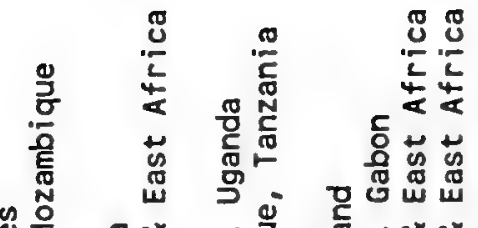

- 感 क ฮ

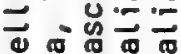

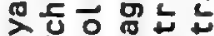

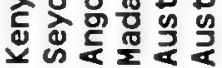

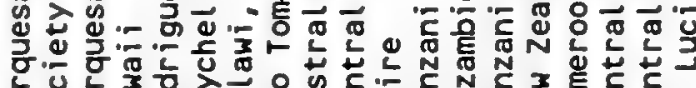

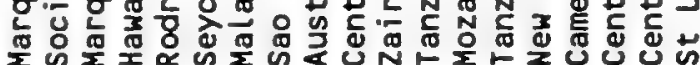

U

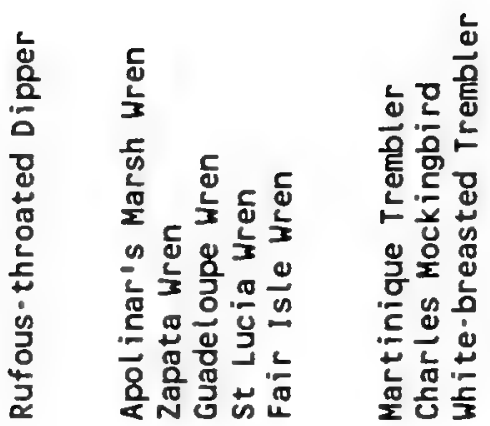

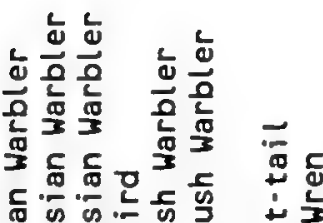

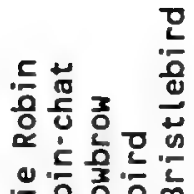

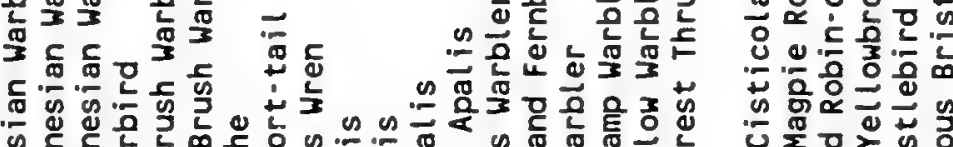

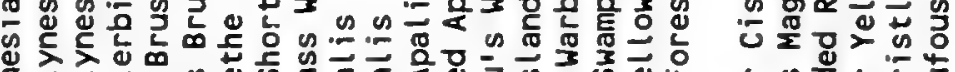

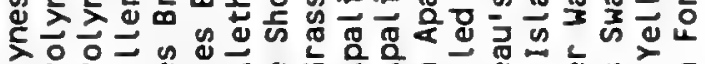

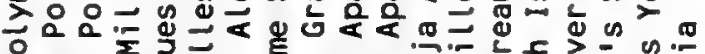

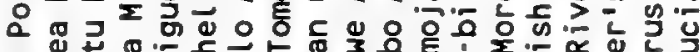

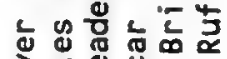

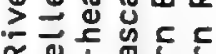
๔

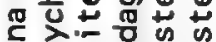

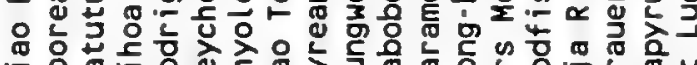

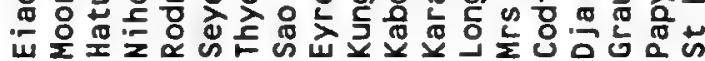

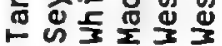

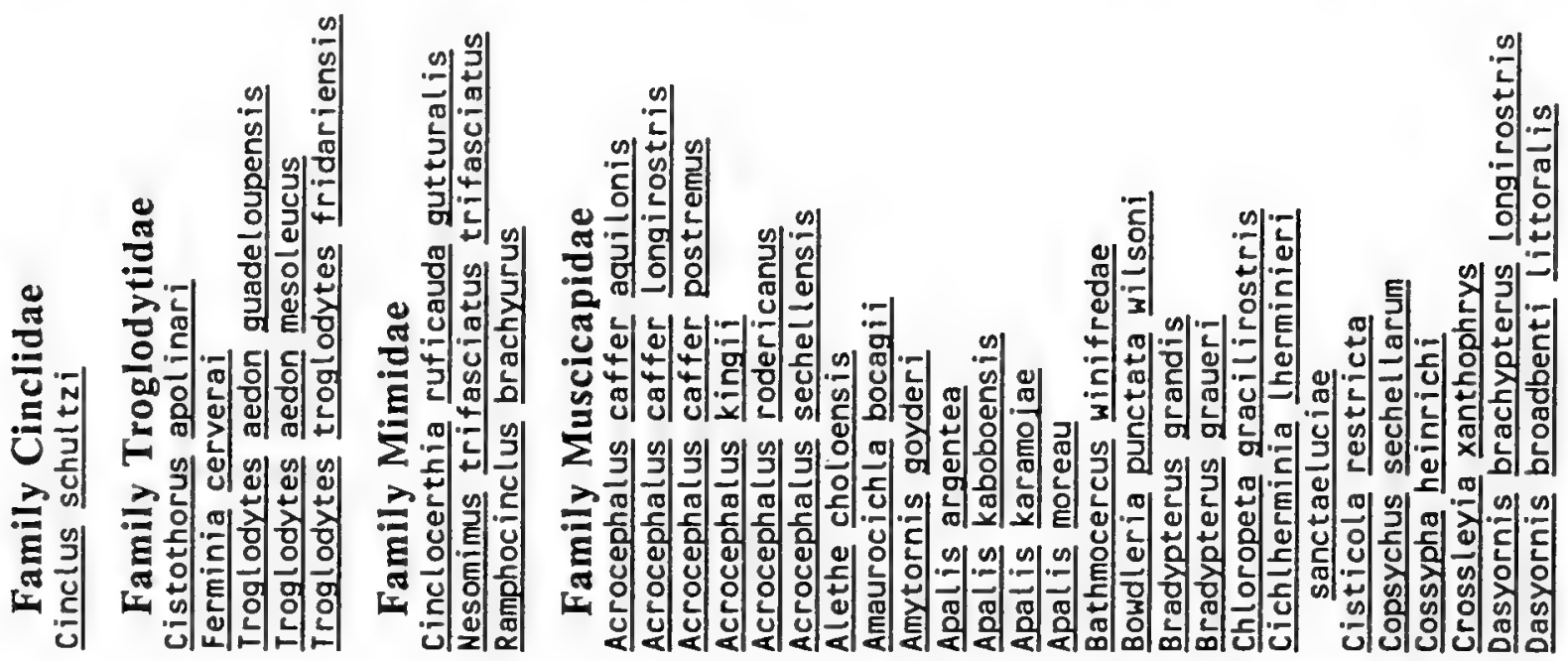




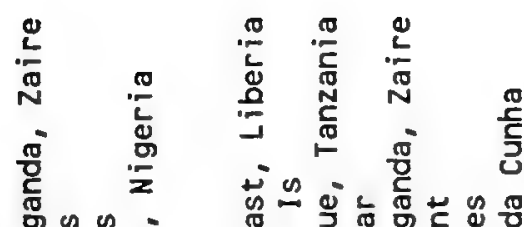

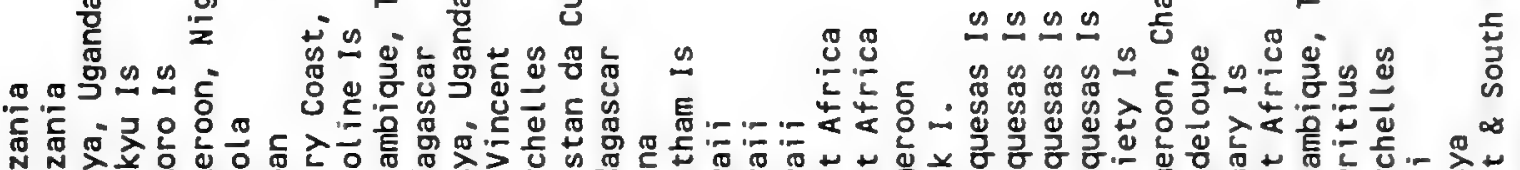

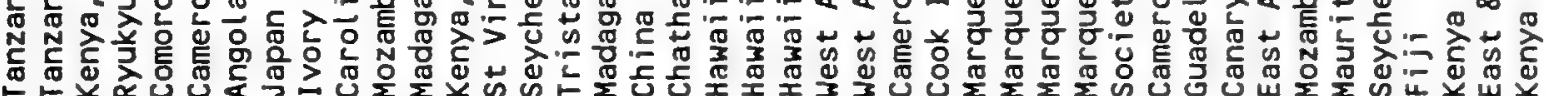

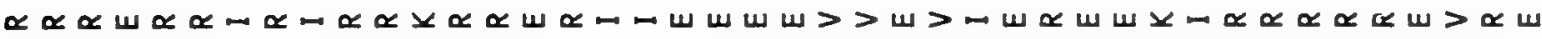

$\frac{\frac{1}{0}}{\frac{0}{0}}$

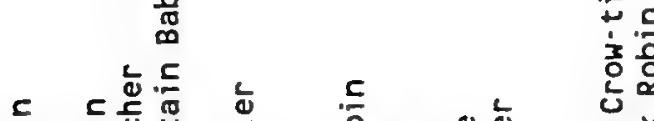

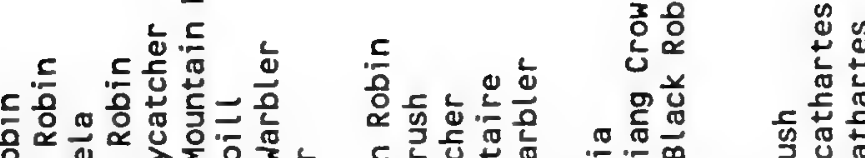

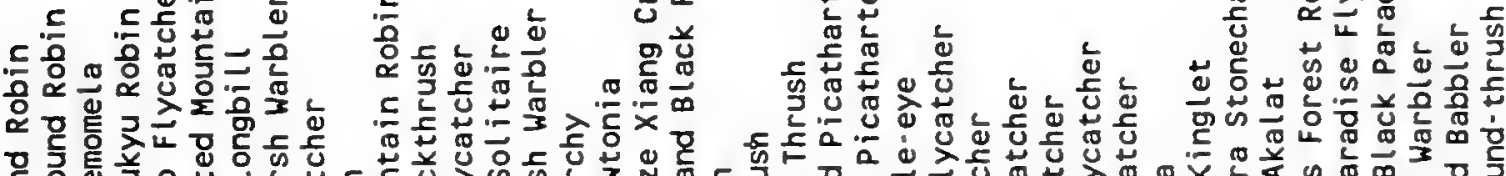

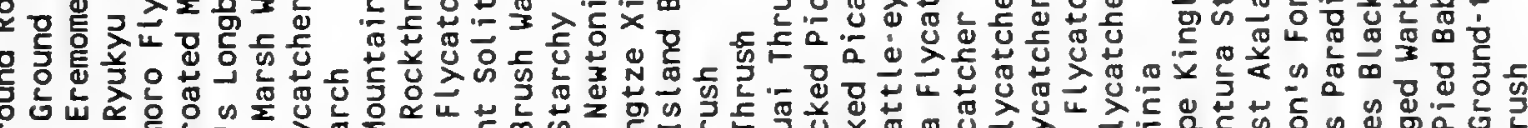

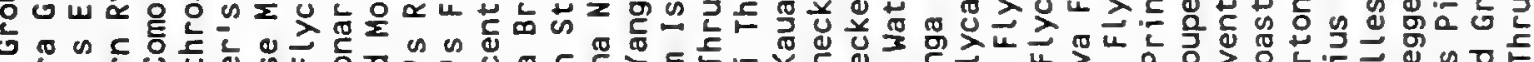

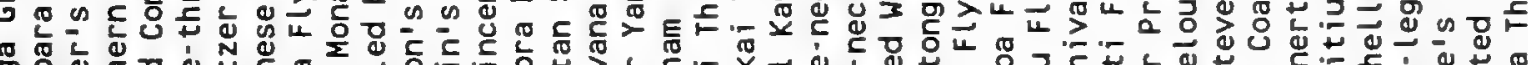

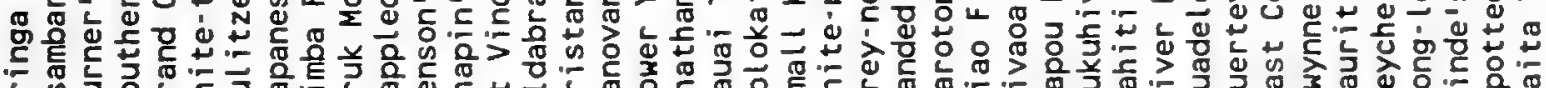

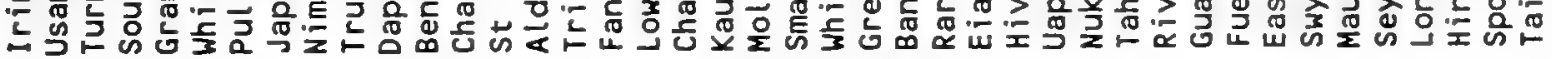

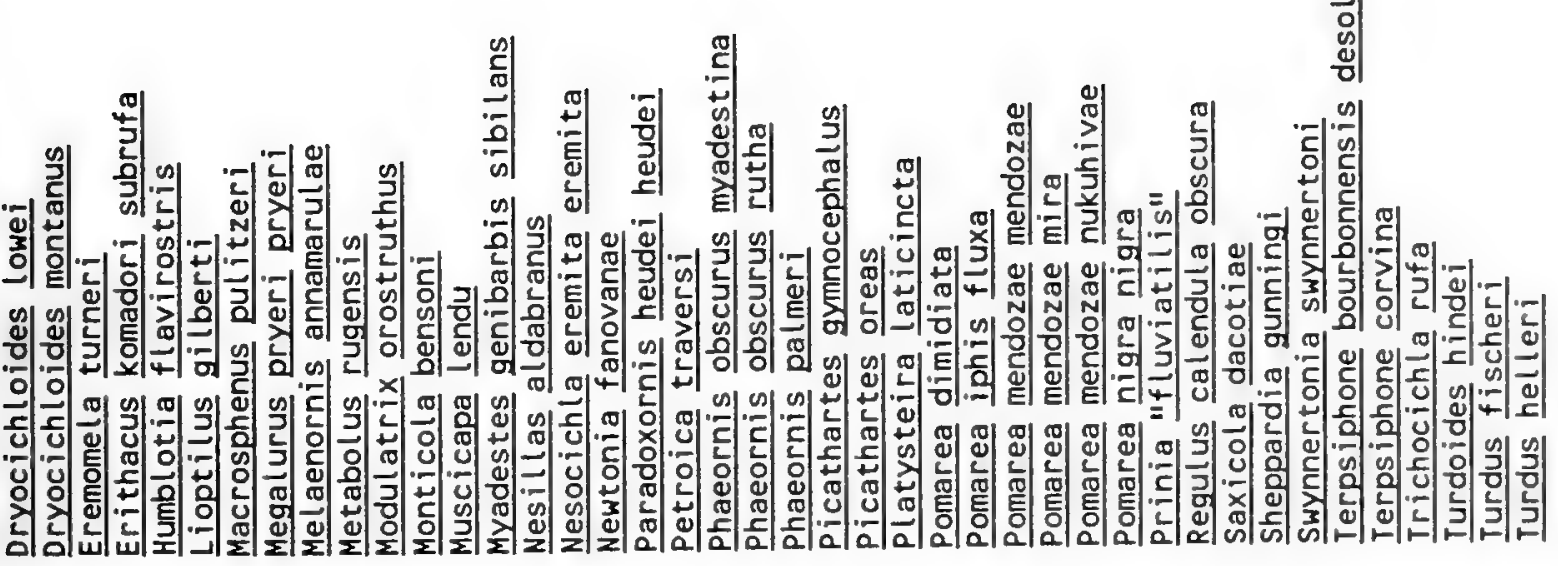




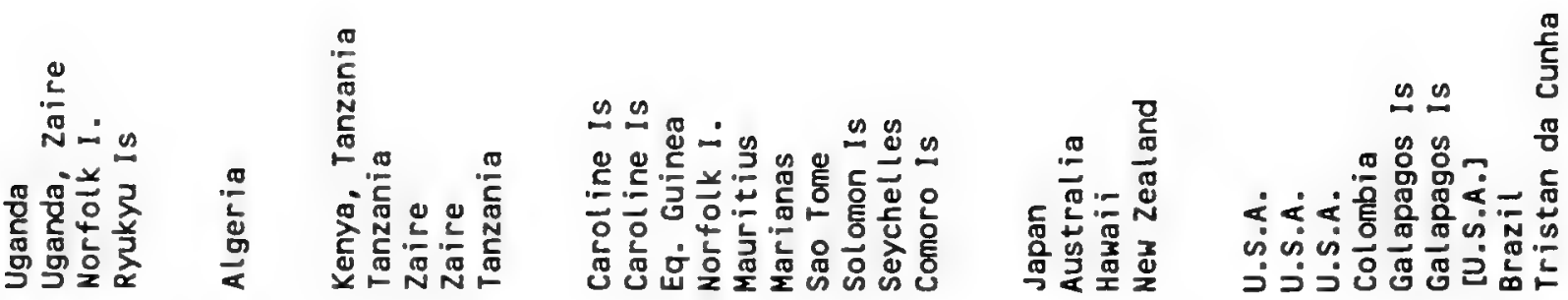

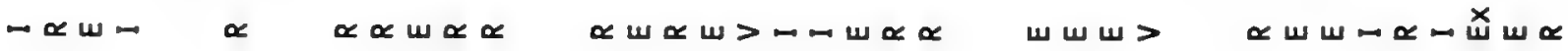

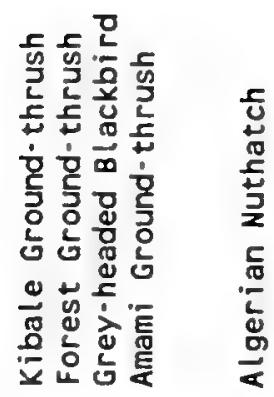
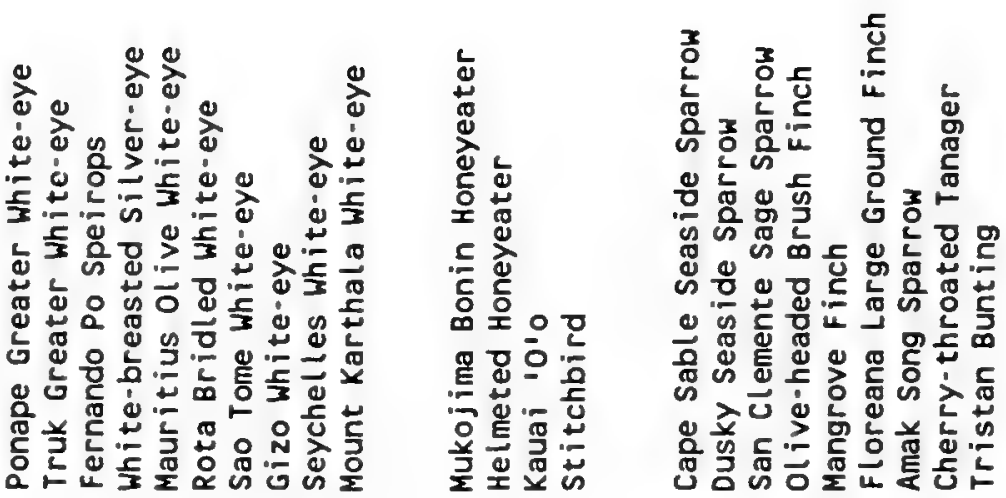

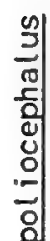

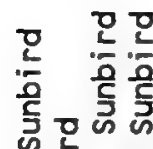
은

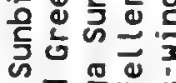

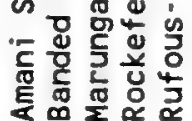

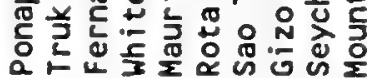

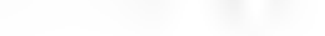

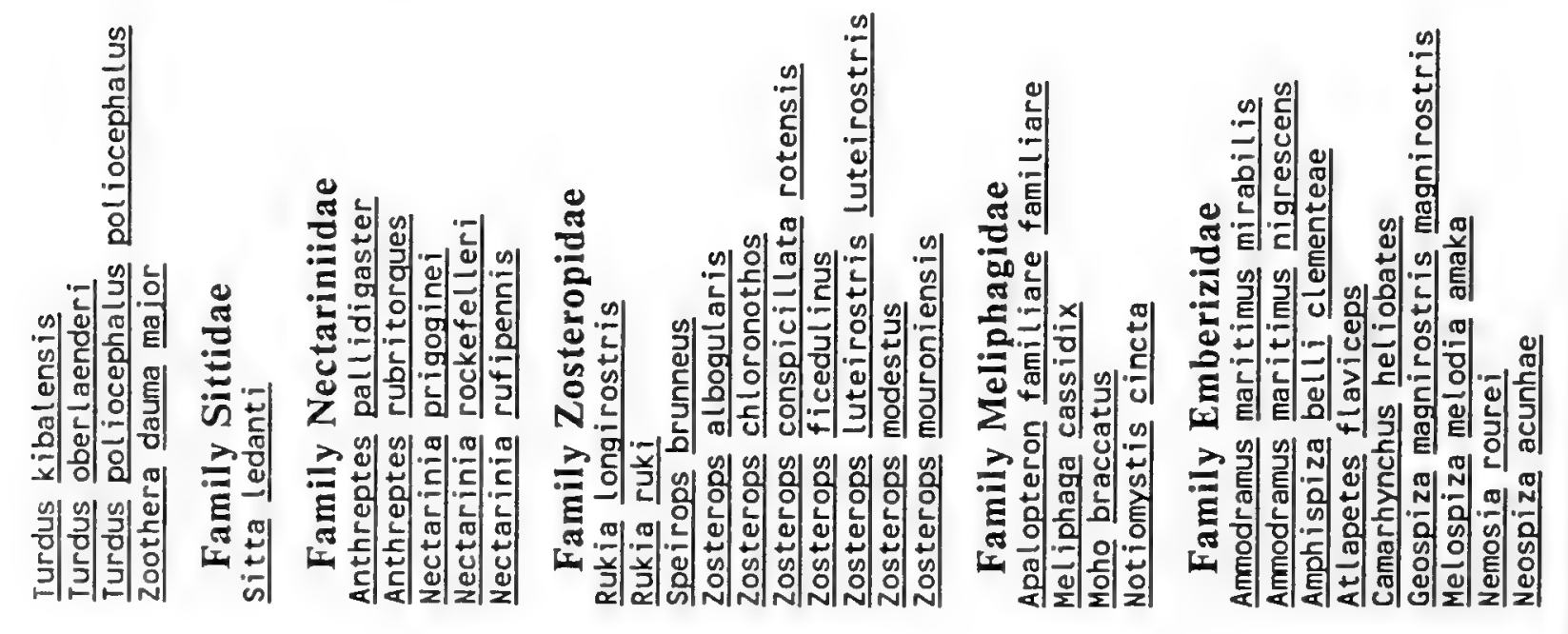




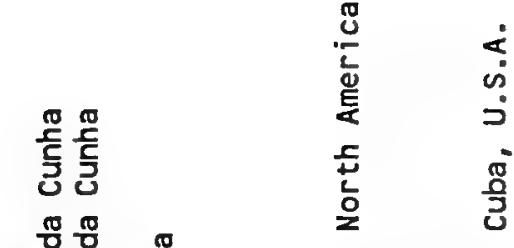

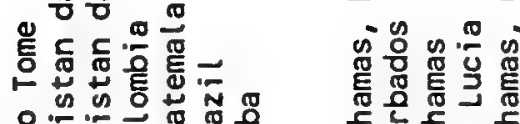

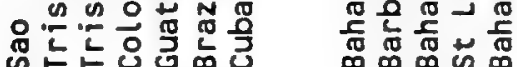

两品品的更

山ッ山

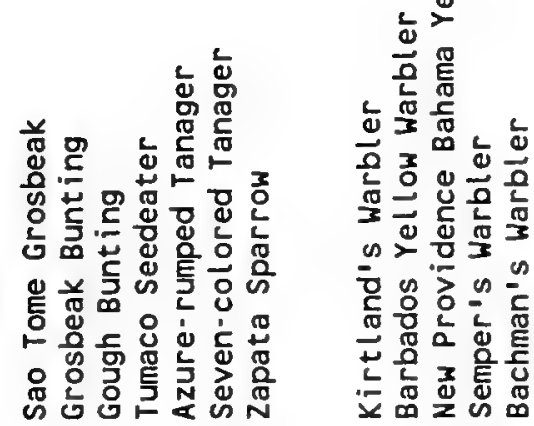

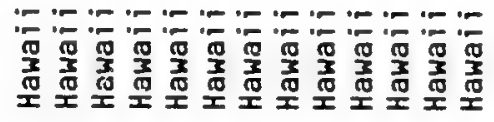

$w w w>w \propto>\propto w \omega>w$

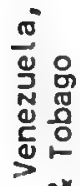

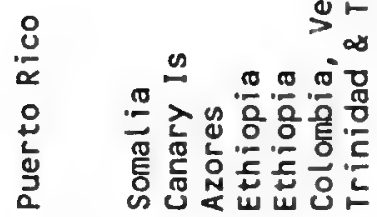

$\propto \propto \omega \propto \omega$

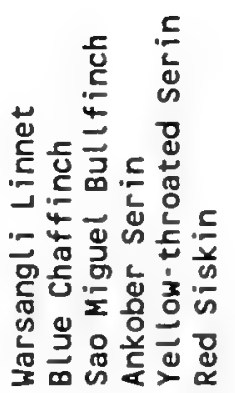

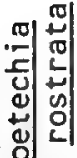

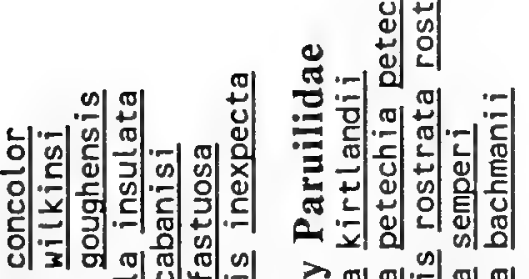

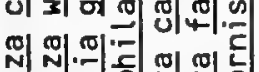

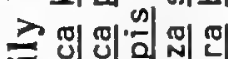
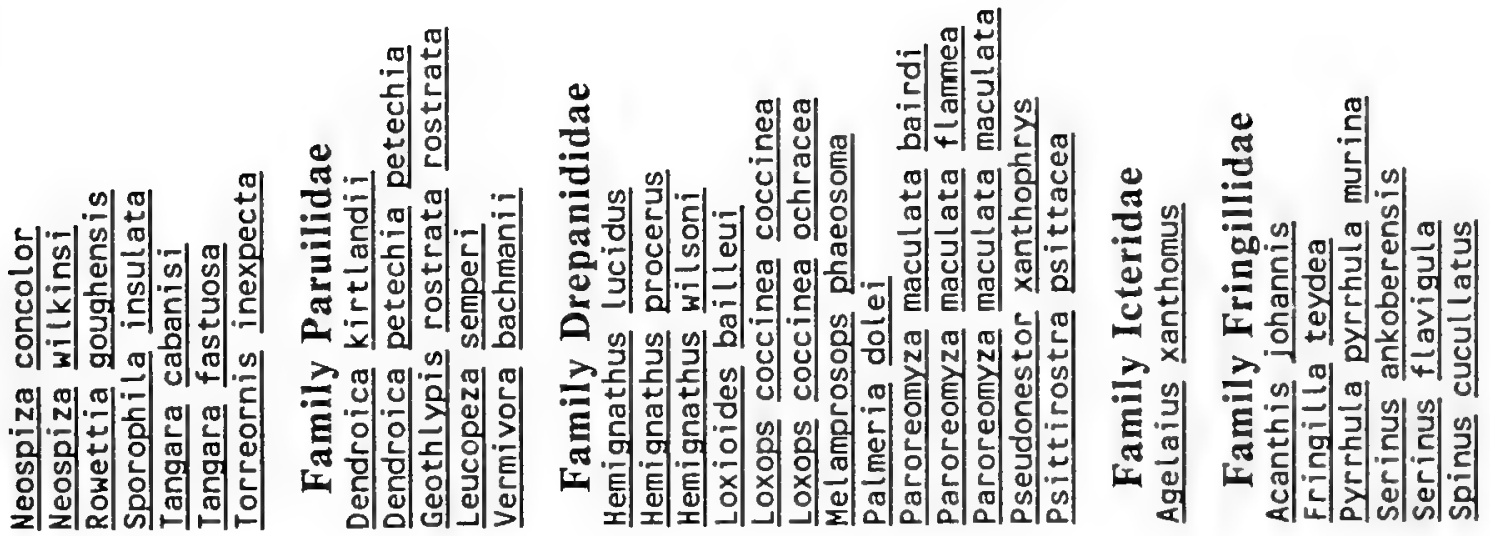


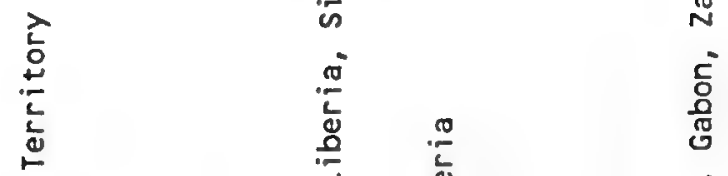
$\frac{10}{20}$

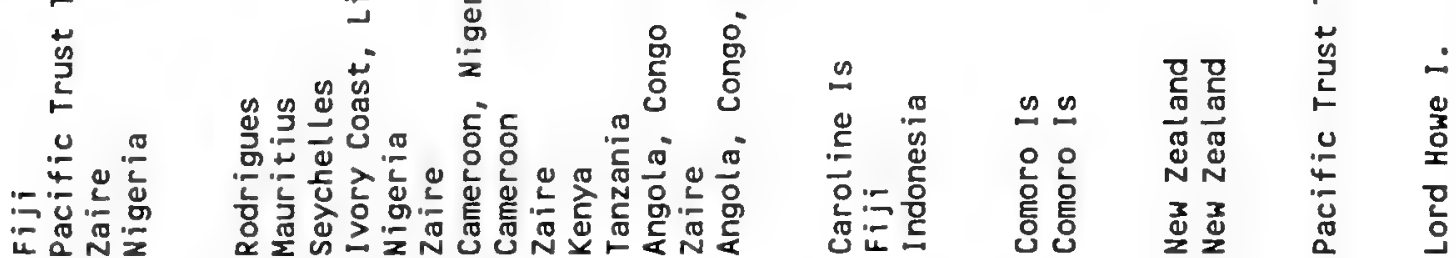

$x \sim x x$

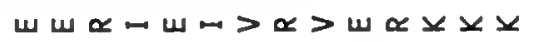

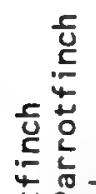

은

造过

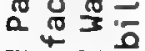

월 원 三丨

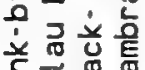

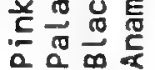

$\propto x$

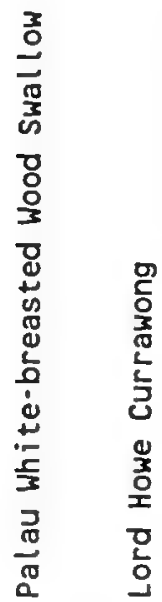

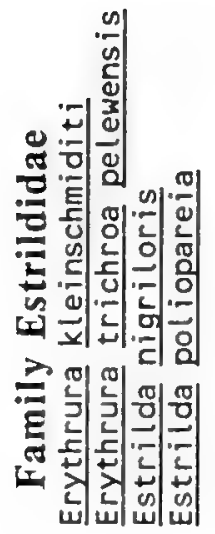

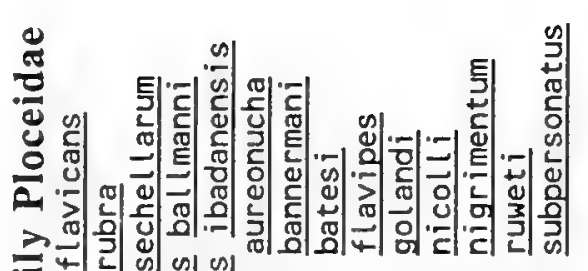

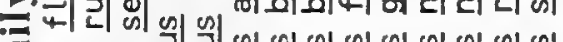

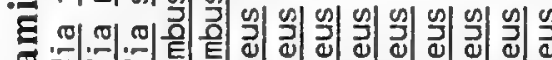

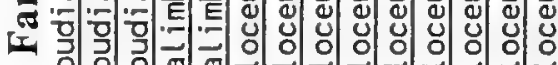
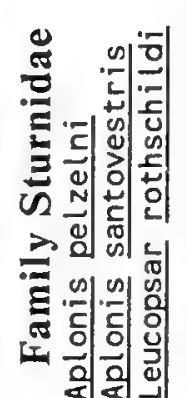

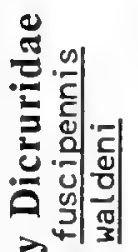

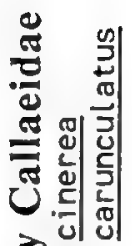

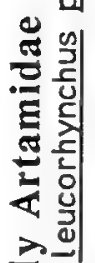

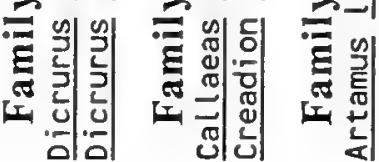

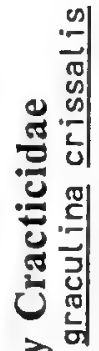

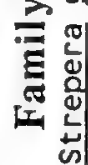




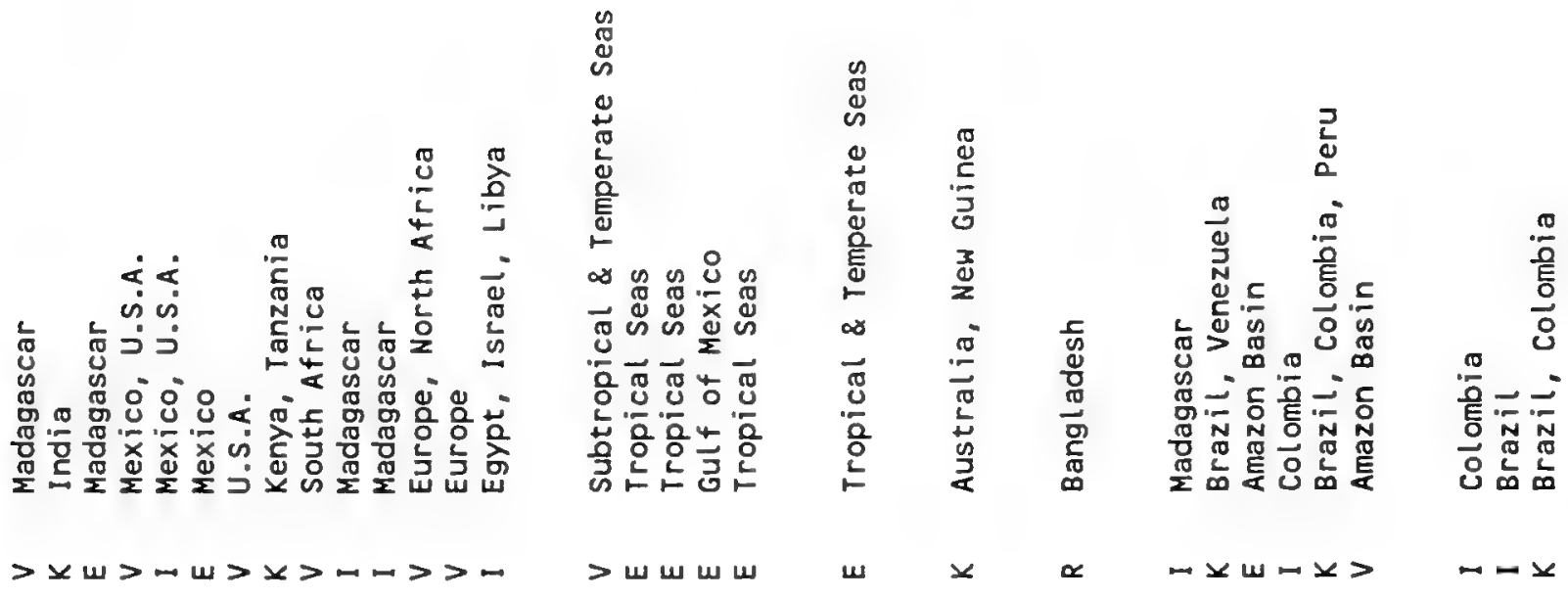

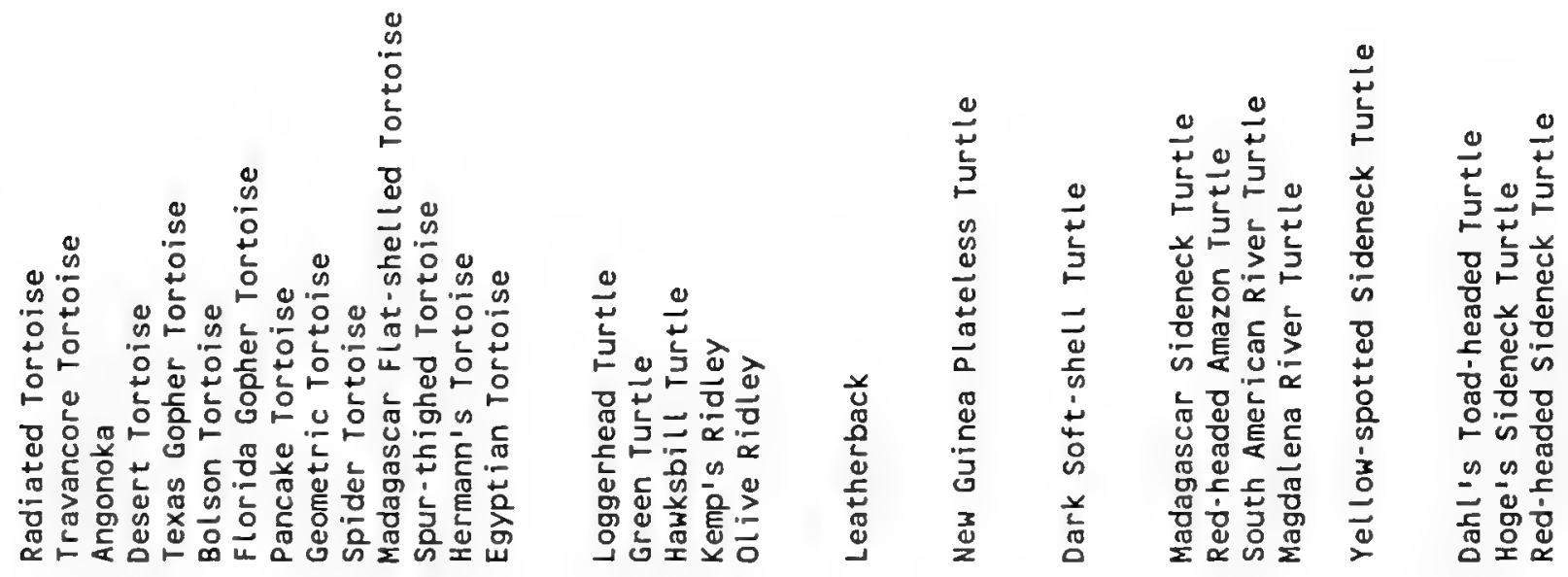
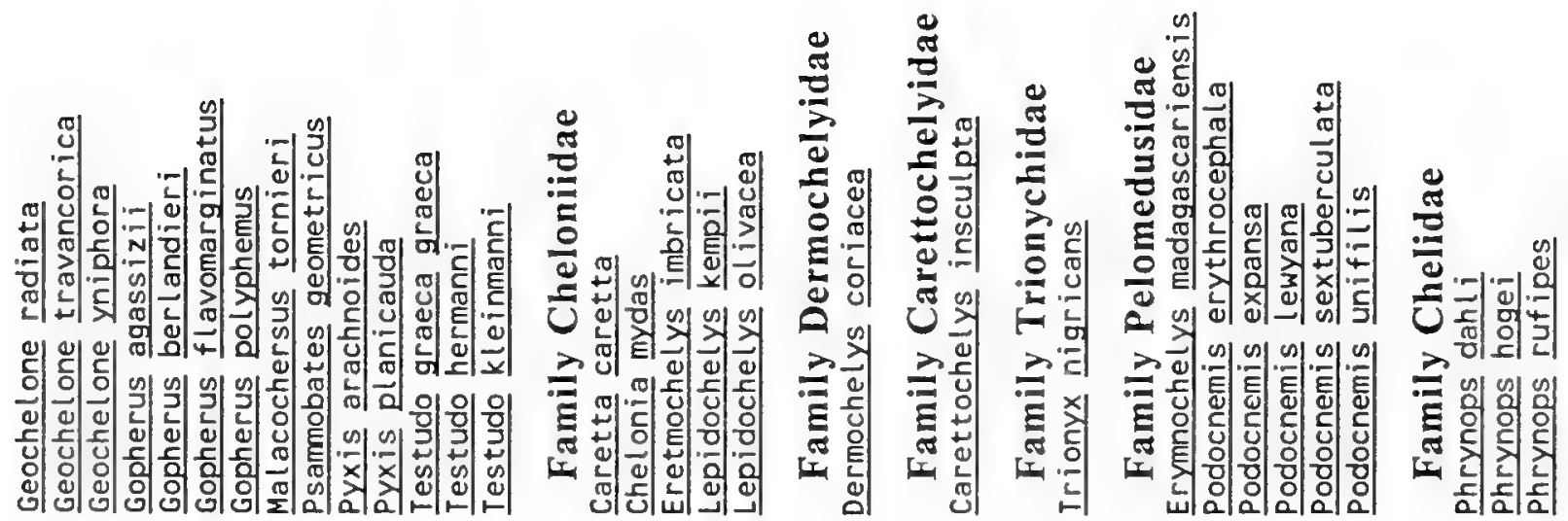


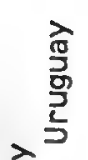

\ֶ,

$\operatorname{8in}^{\circ}=\frac{0}{2}$

$\frac{10}{10}$

वृ

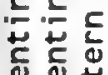

ơ

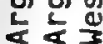

$\alpha \geq \mu$

$\stackrel{0}{*}$

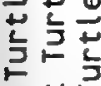

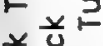

范。

焉

$\frac{8}{6}$

is is c

$\therefore \times$

$\frac{10}{0}$

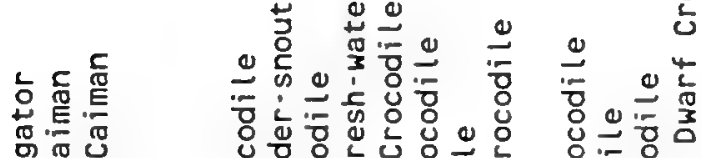

ฮٓ

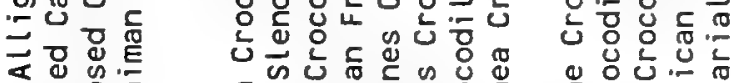

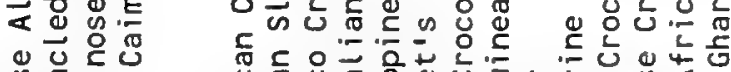

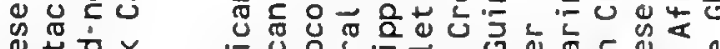

点若兑

它远迹恋

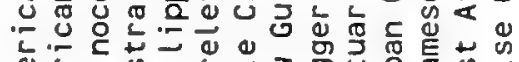

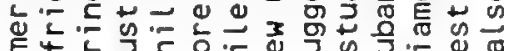

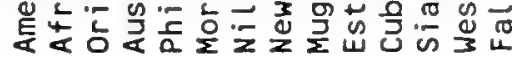

亳

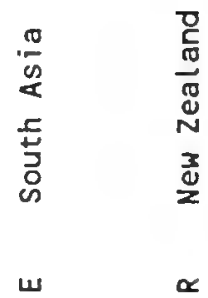

ت.

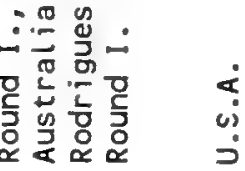

$\propto>\operatorname{lin} \propto \alpha$

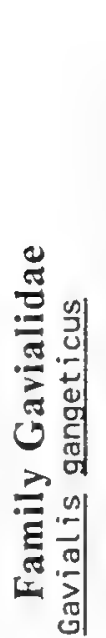

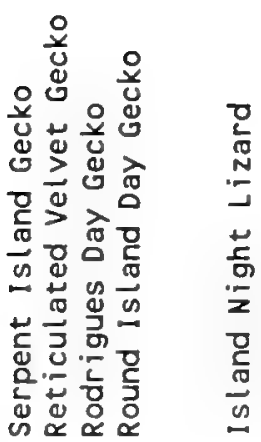
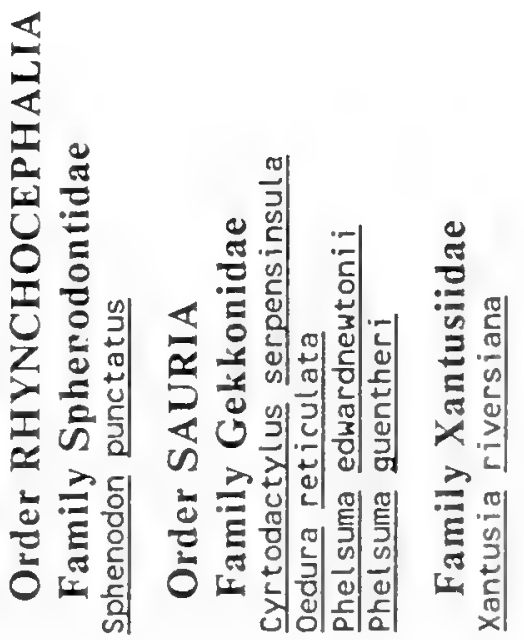


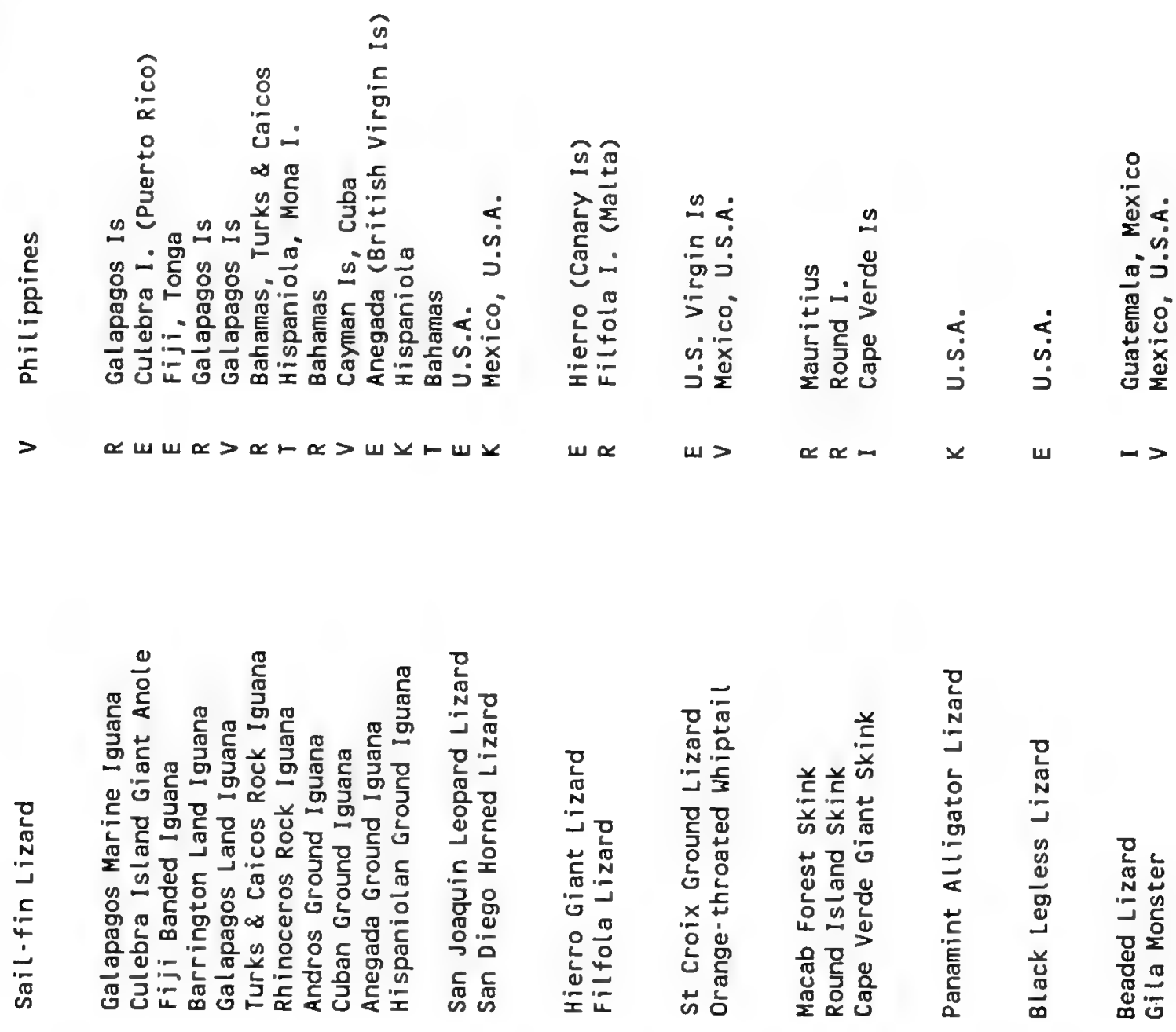
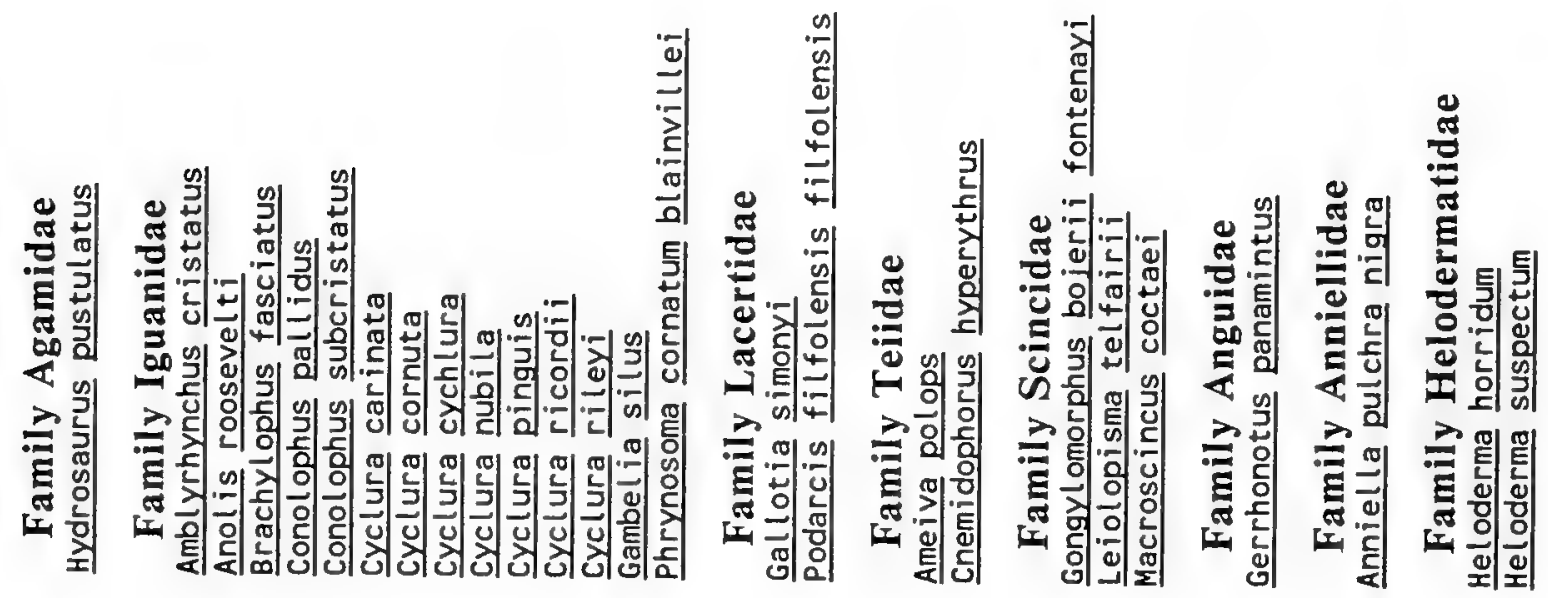


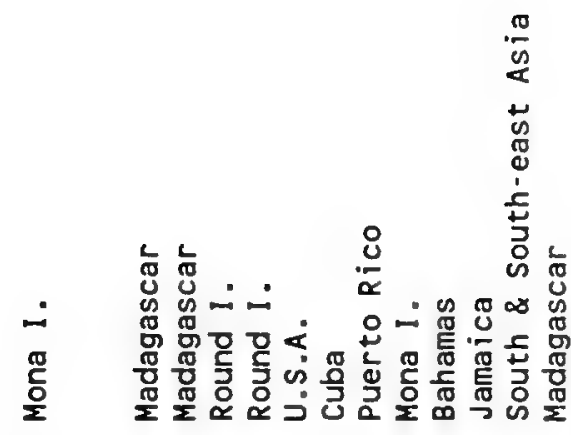

$>\propto$

$\propto$
능

동

'פ

$\overline{0}$

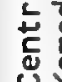

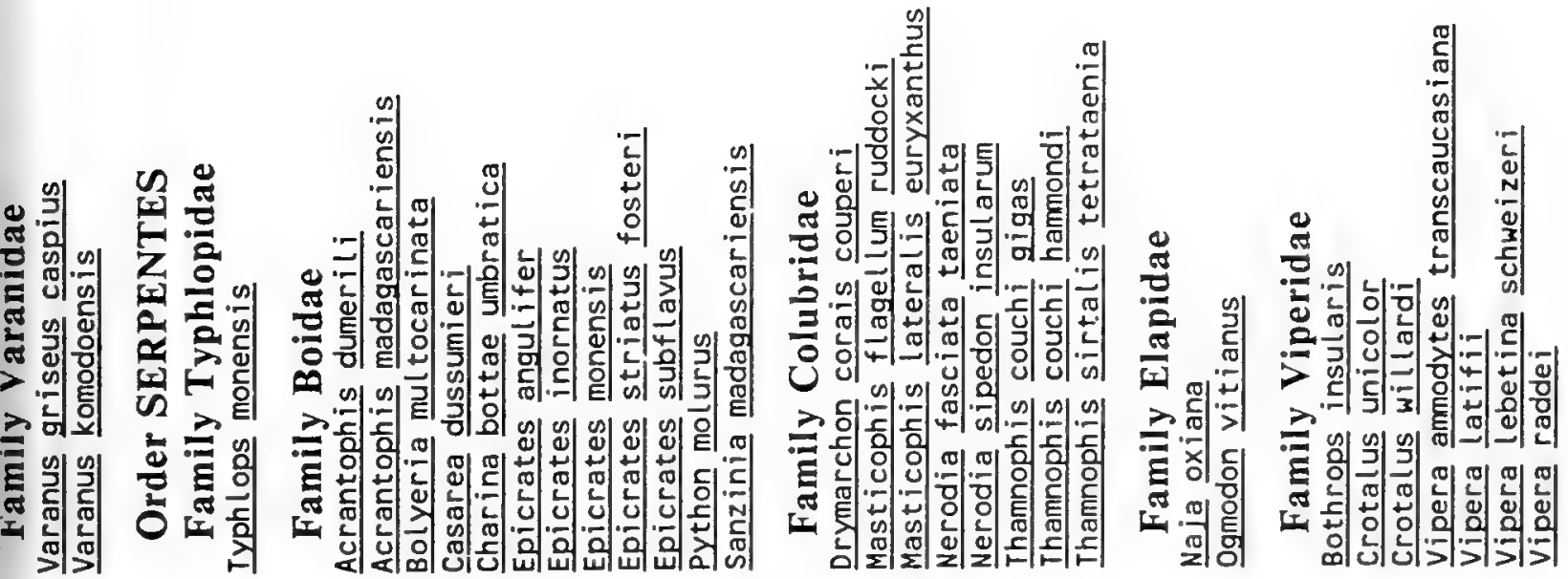

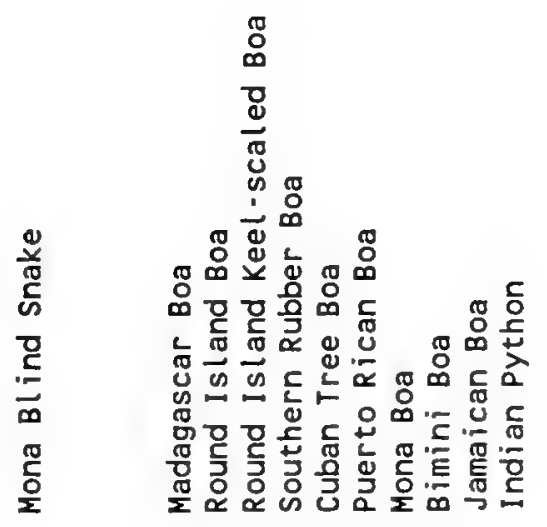

旁

$\frac{n}{\stackrel{0}{a}}$

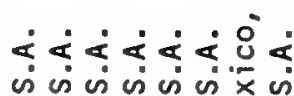

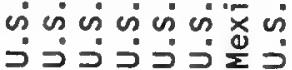

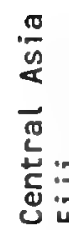

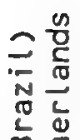

$\dot{5}$
$\dot{0}$
$\dot{0}$
$\dot{8}<$
$\frac{0}{2}$

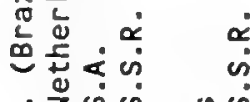
$\because Z$ ñ

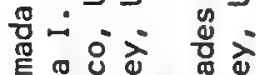

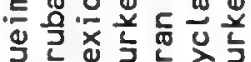

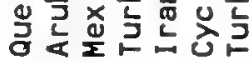

$>\propto \alpha \propto \underline{\alpha \propto \omega}$ $\checkmark \alpha-\alpha \omega>\alpha$

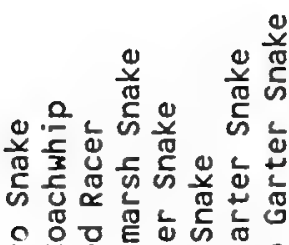

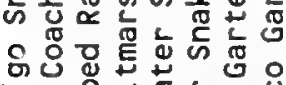

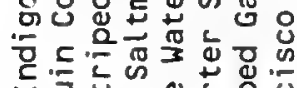

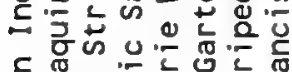

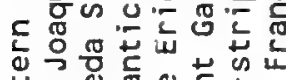

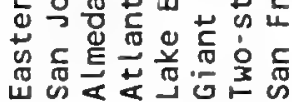

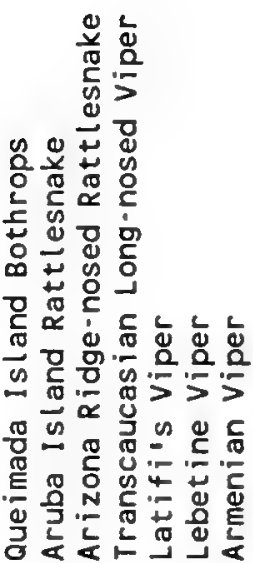




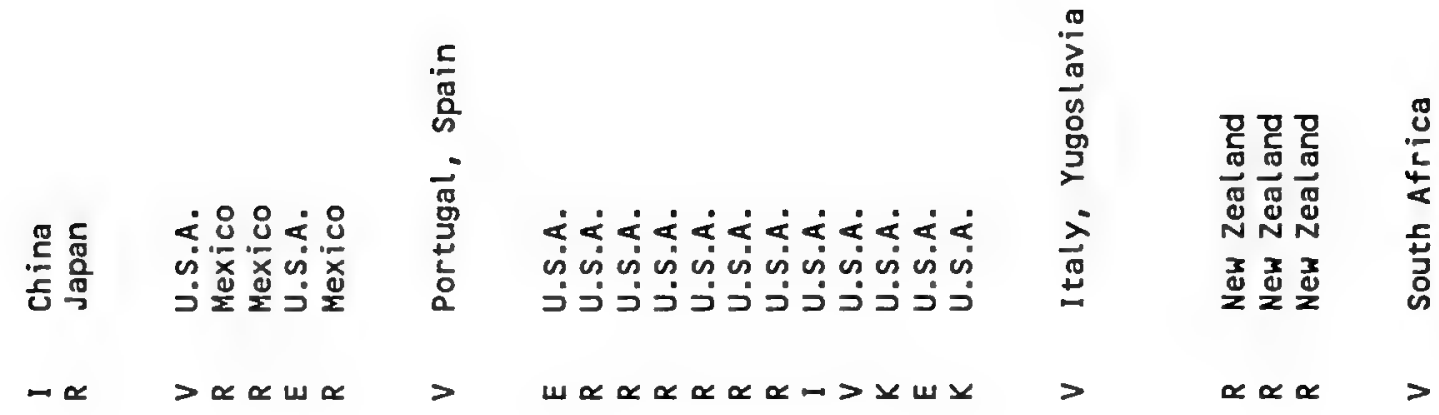
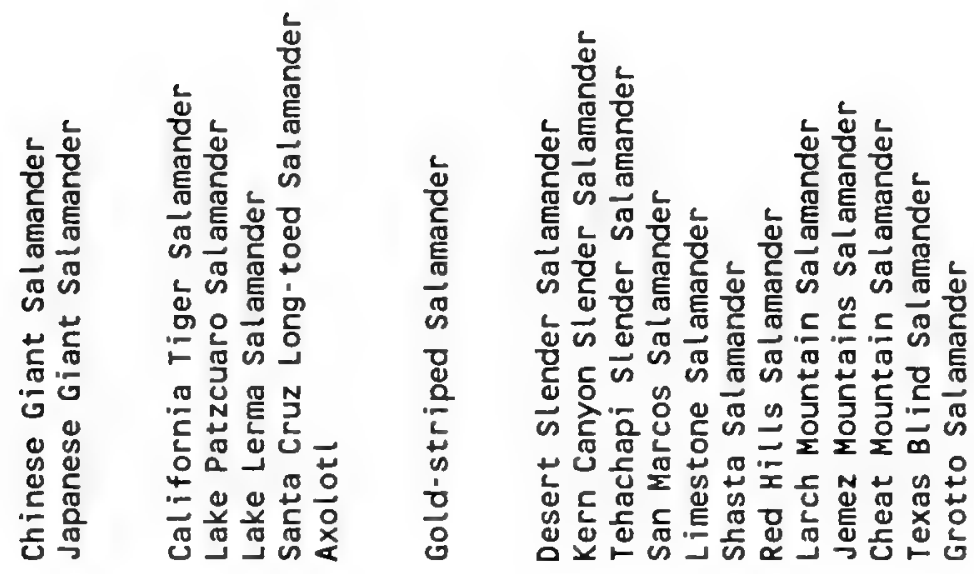

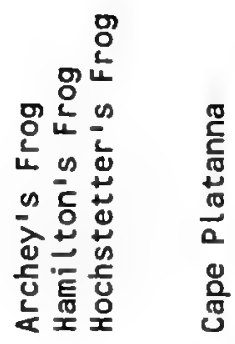
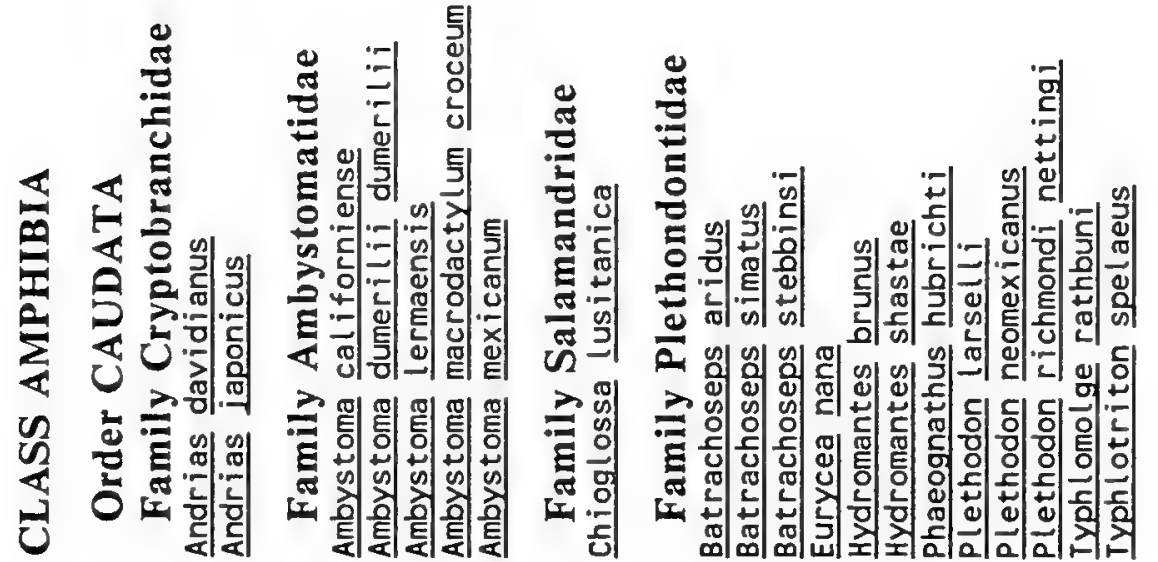

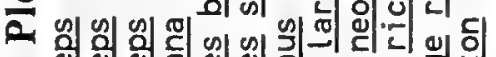
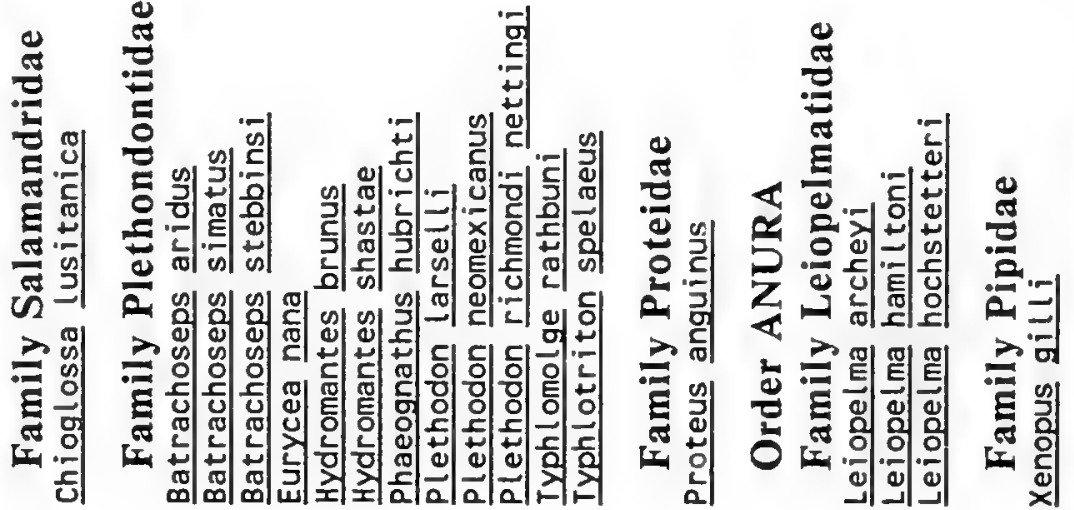

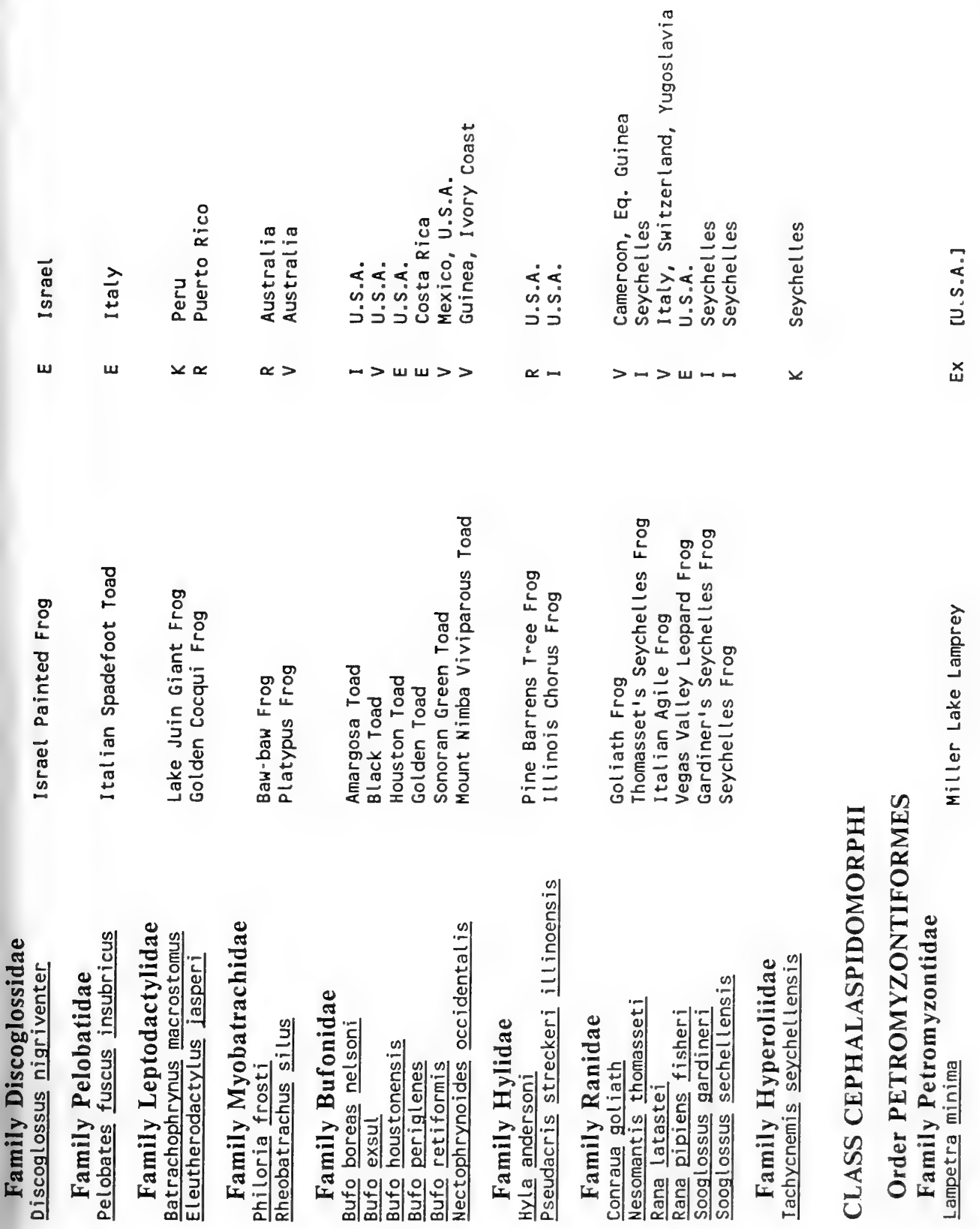


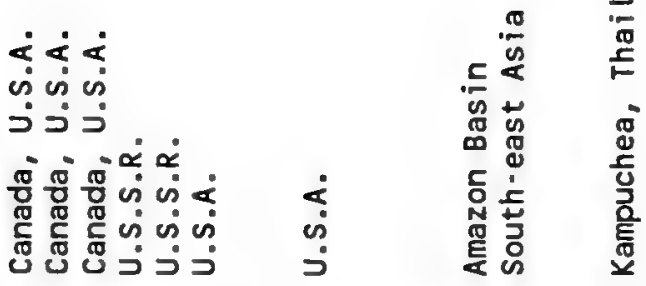

$>>>\propto \omega \quad \sim \propto \propto$
온.

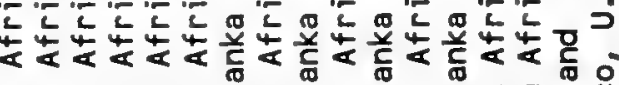

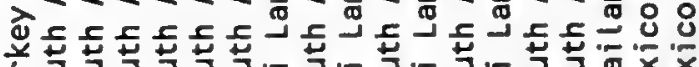

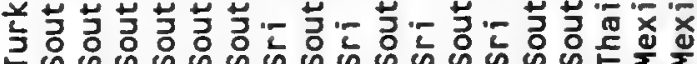

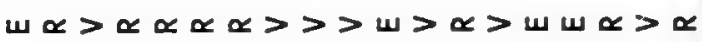

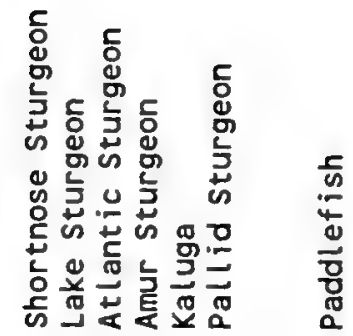

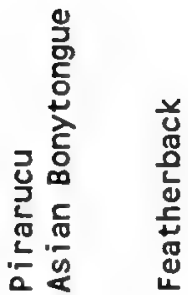
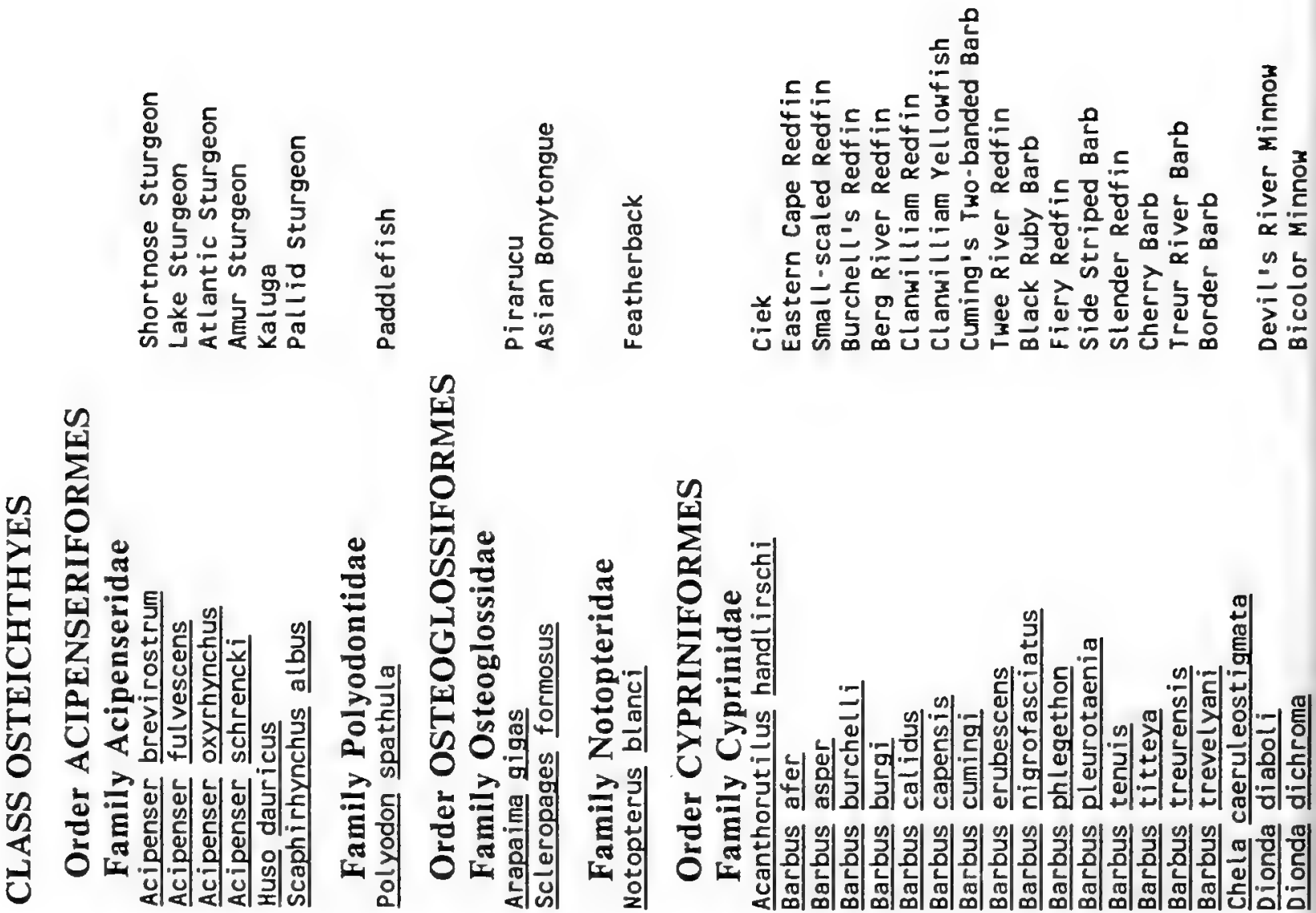

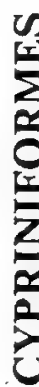

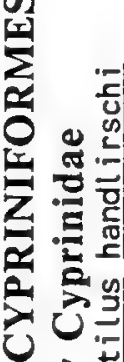

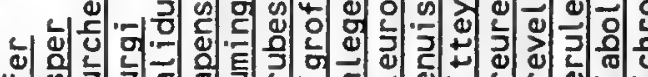

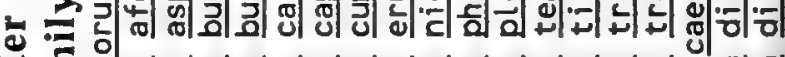

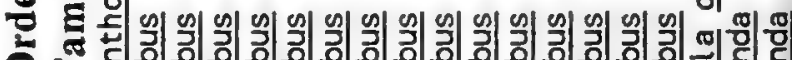

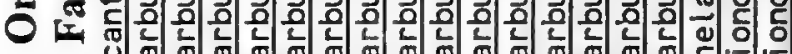

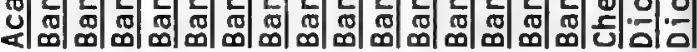




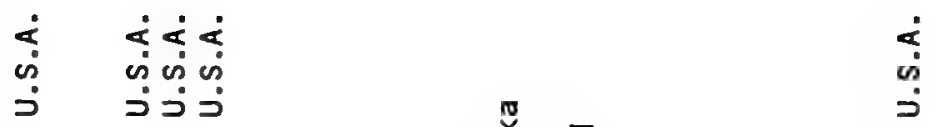

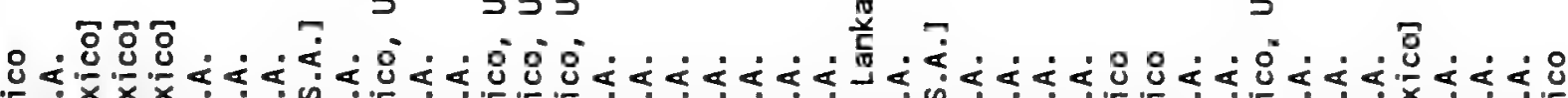

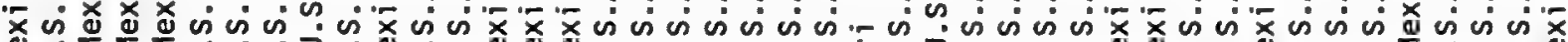

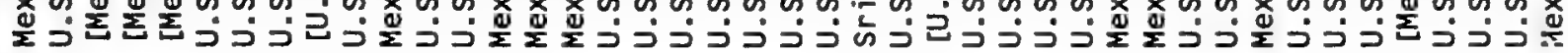

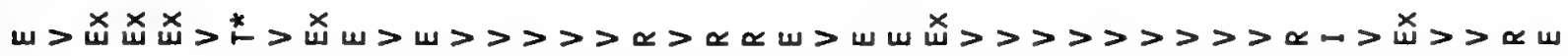

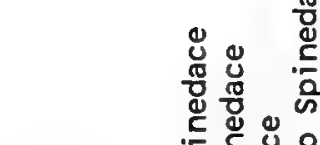

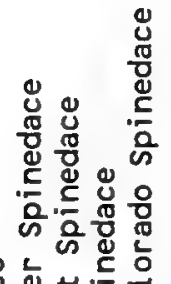

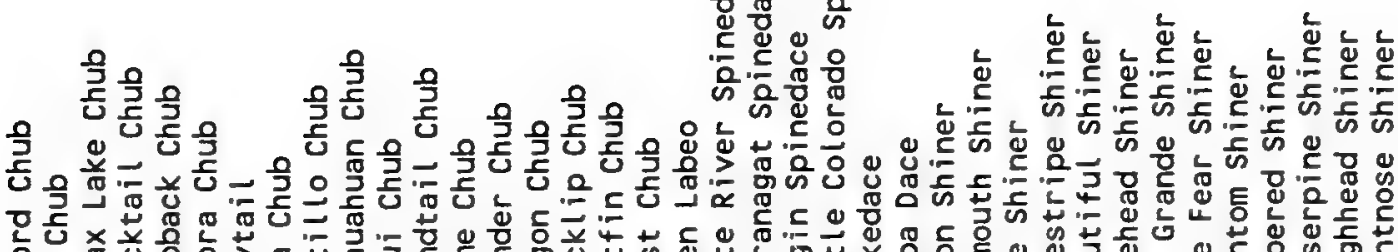

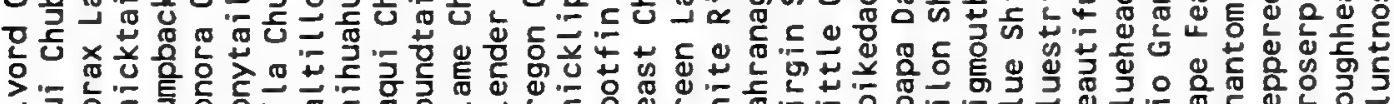

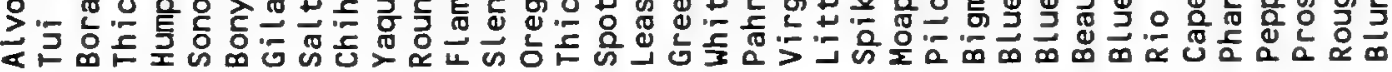

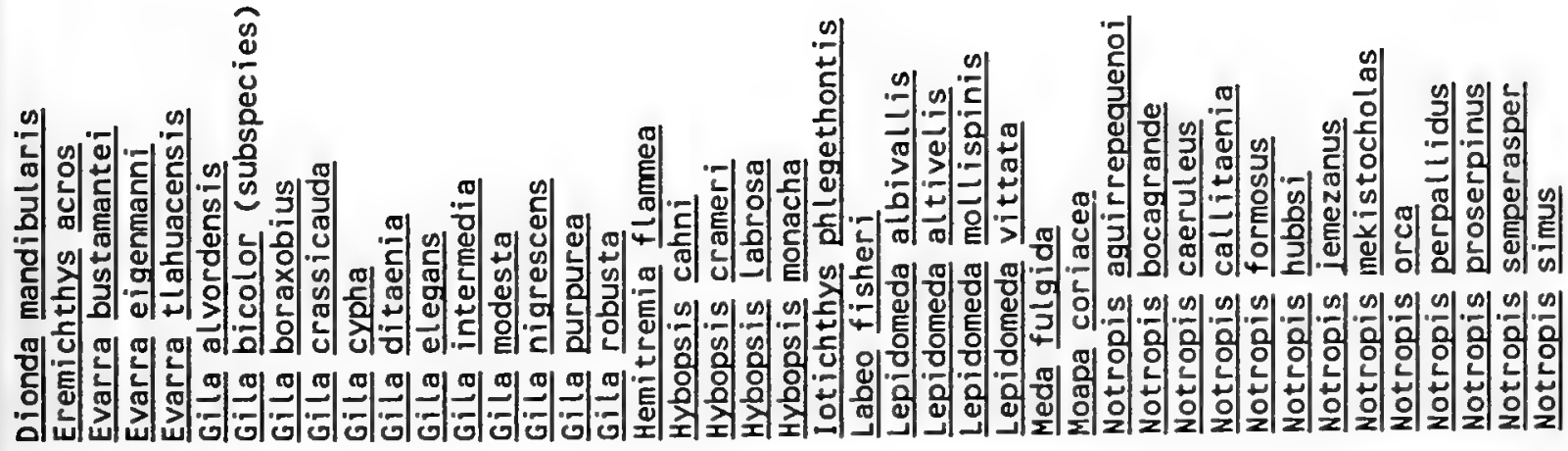




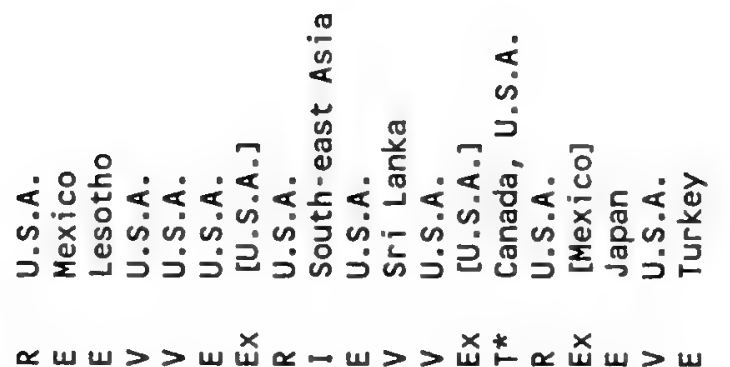

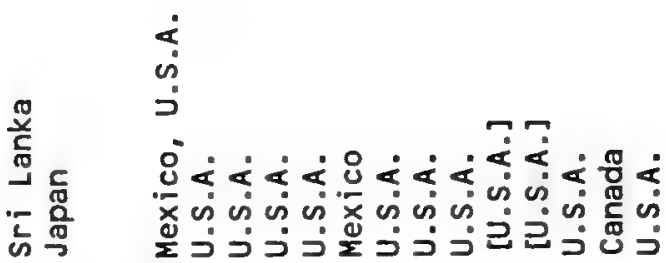

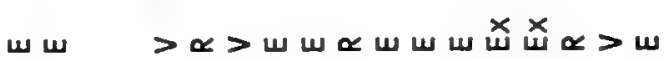

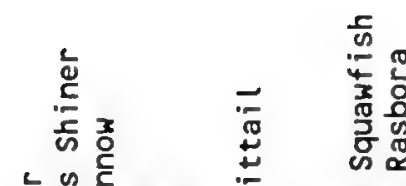

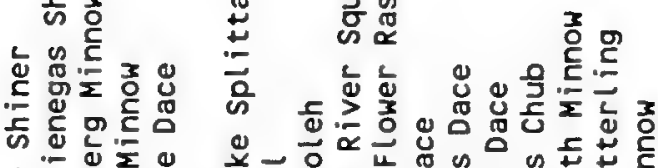

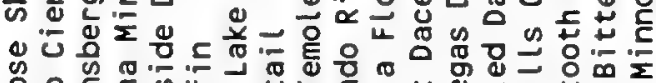
为

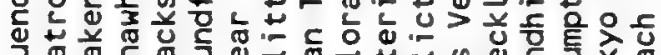

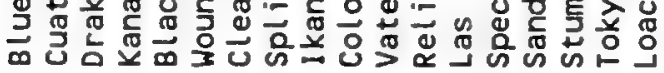

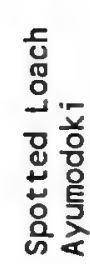

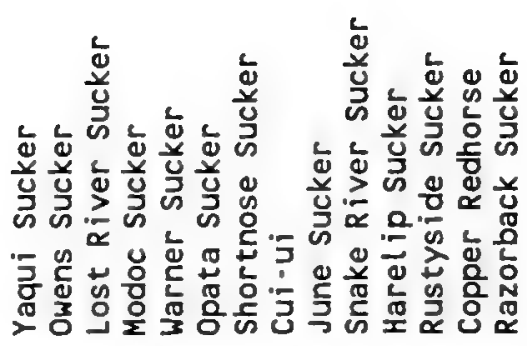

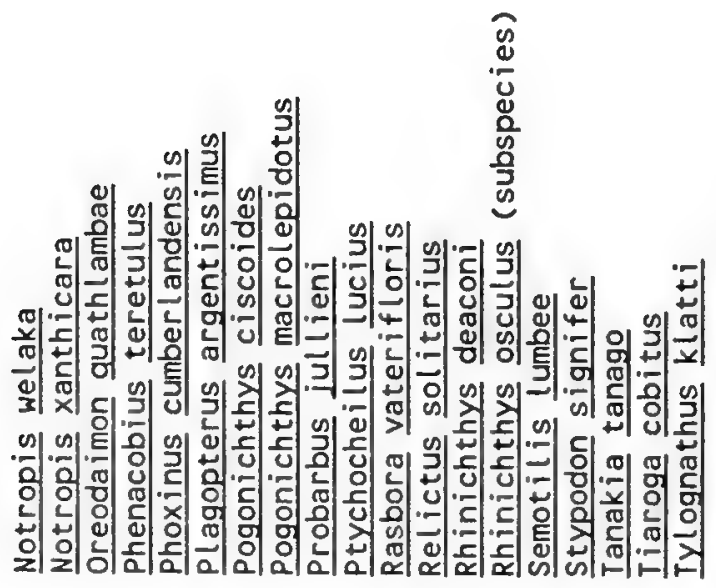

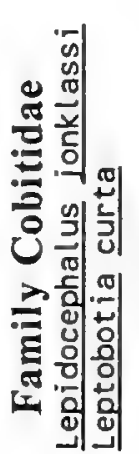

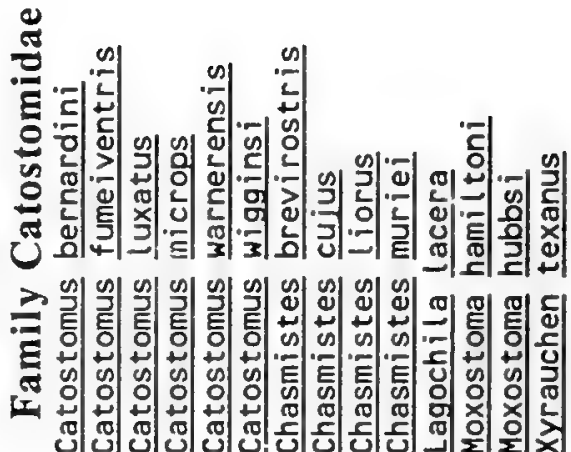



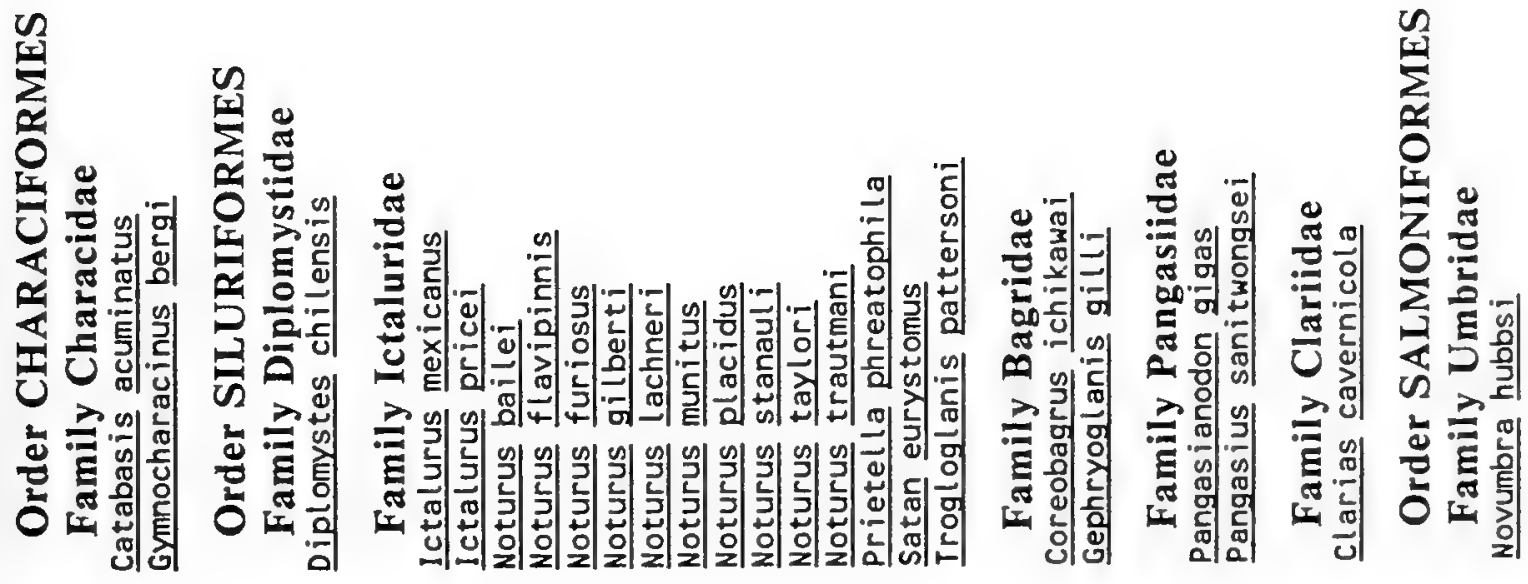

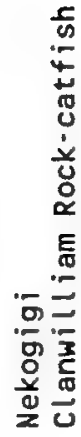

造

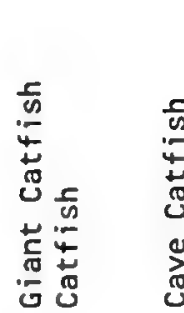




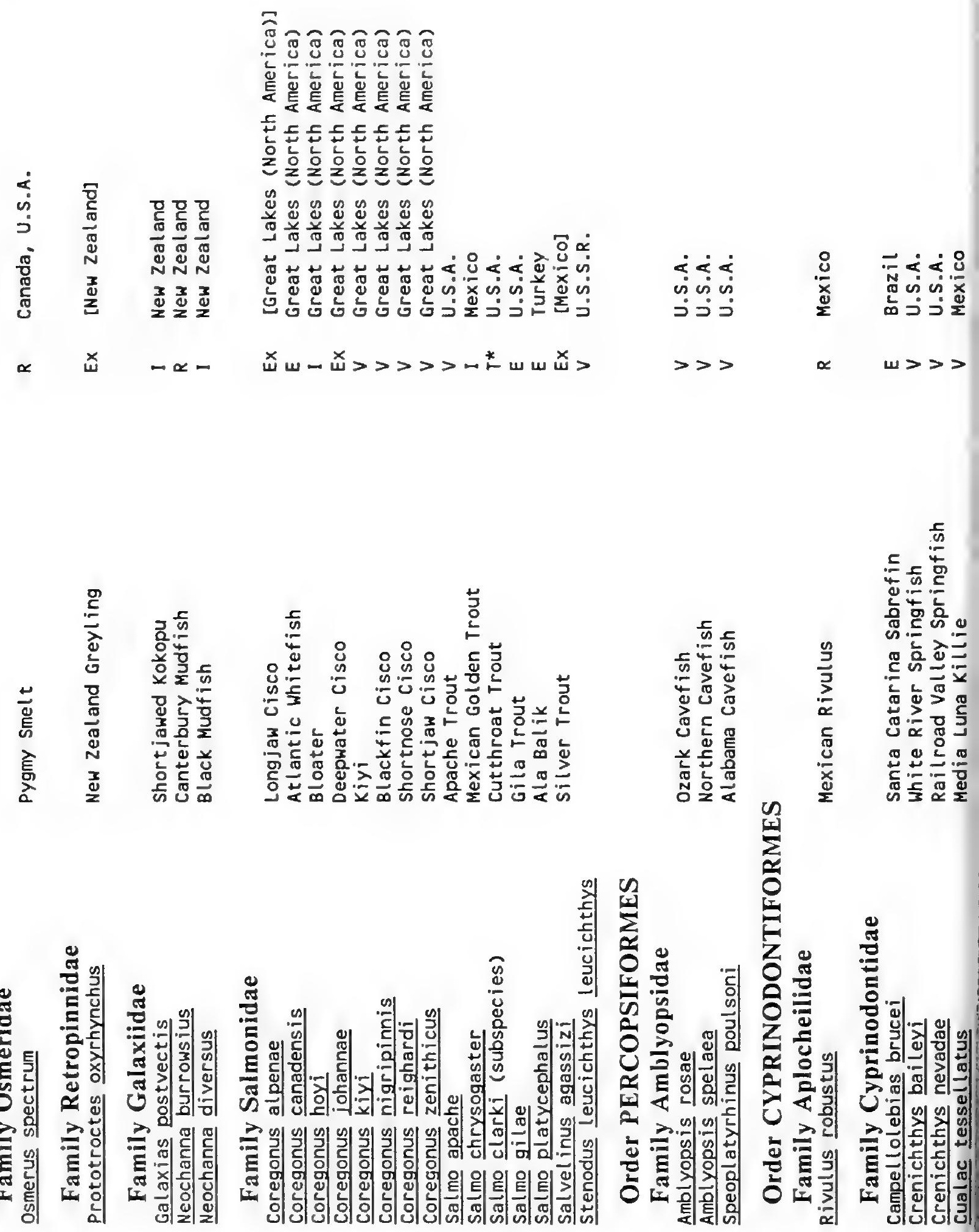




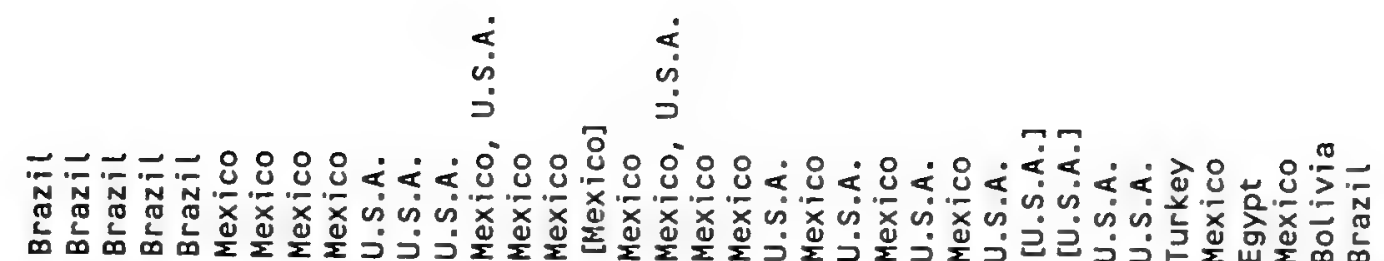

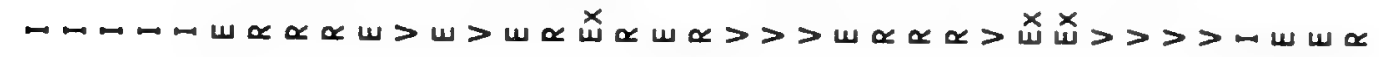
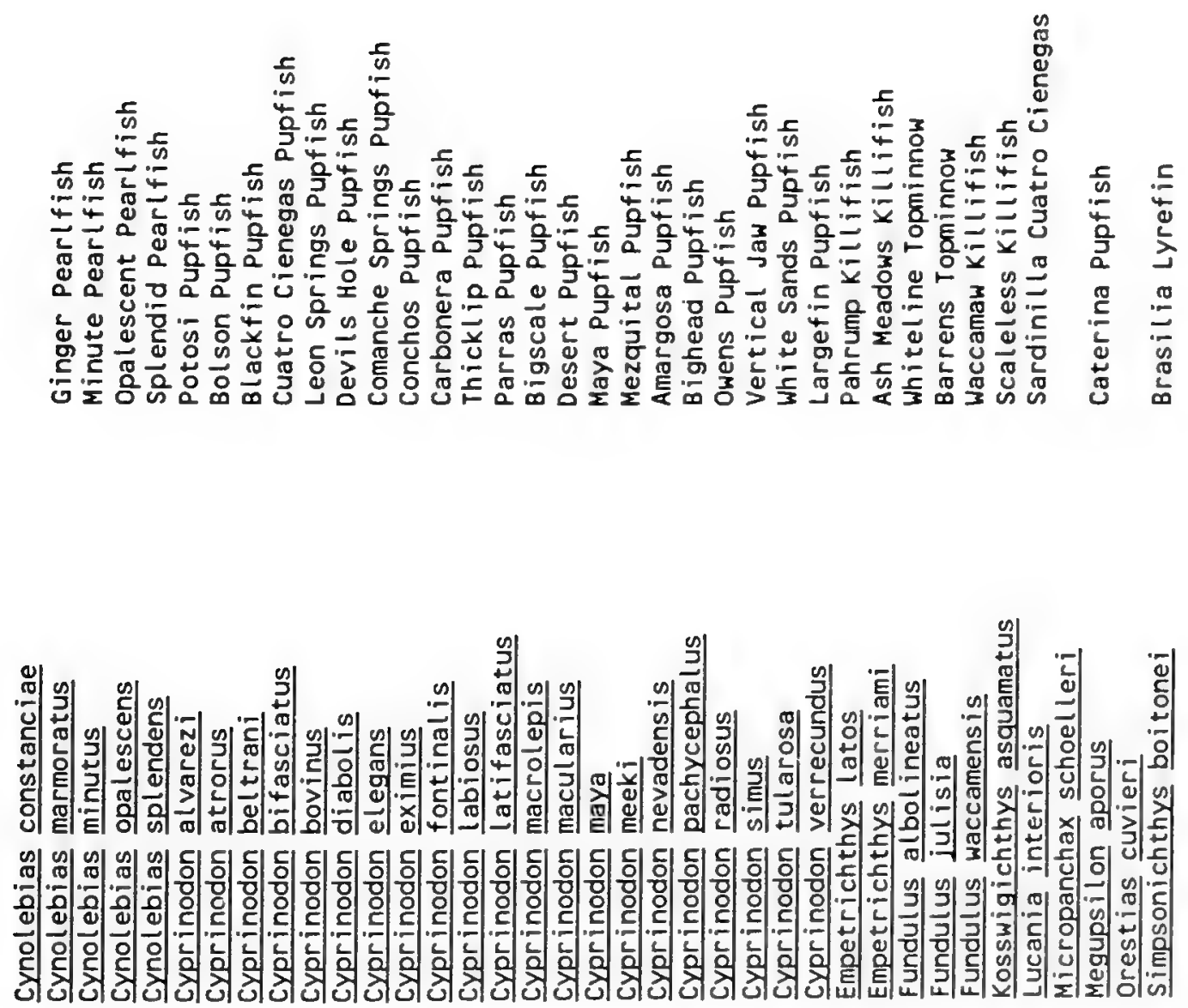
这

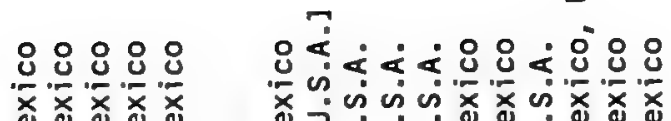

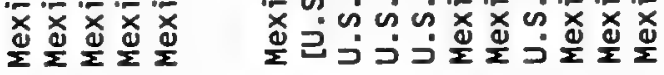

$>>w \propto$

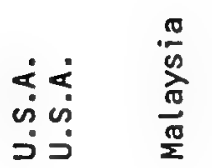

$\infty>\quad \mapsto$

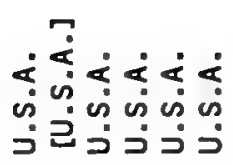

$\propto \stackrel{x}{w} \propto>\propto>$

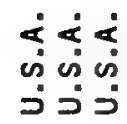

$\alpha \propto>$

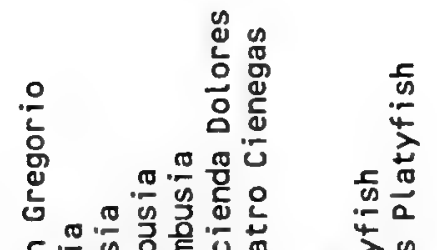

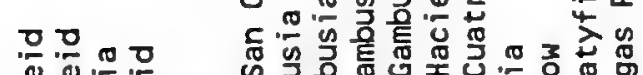

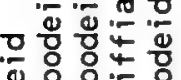
过 ○ $x$

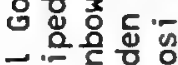

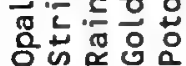

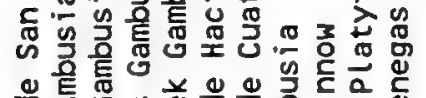

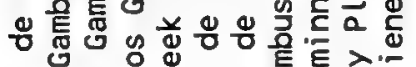

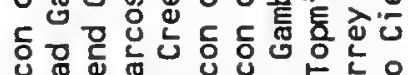

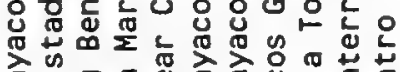

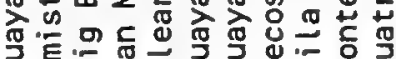

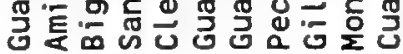

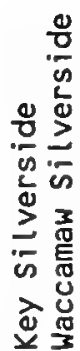

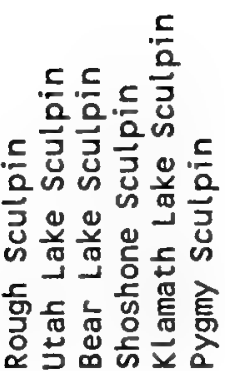

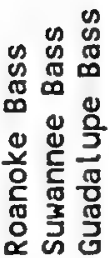
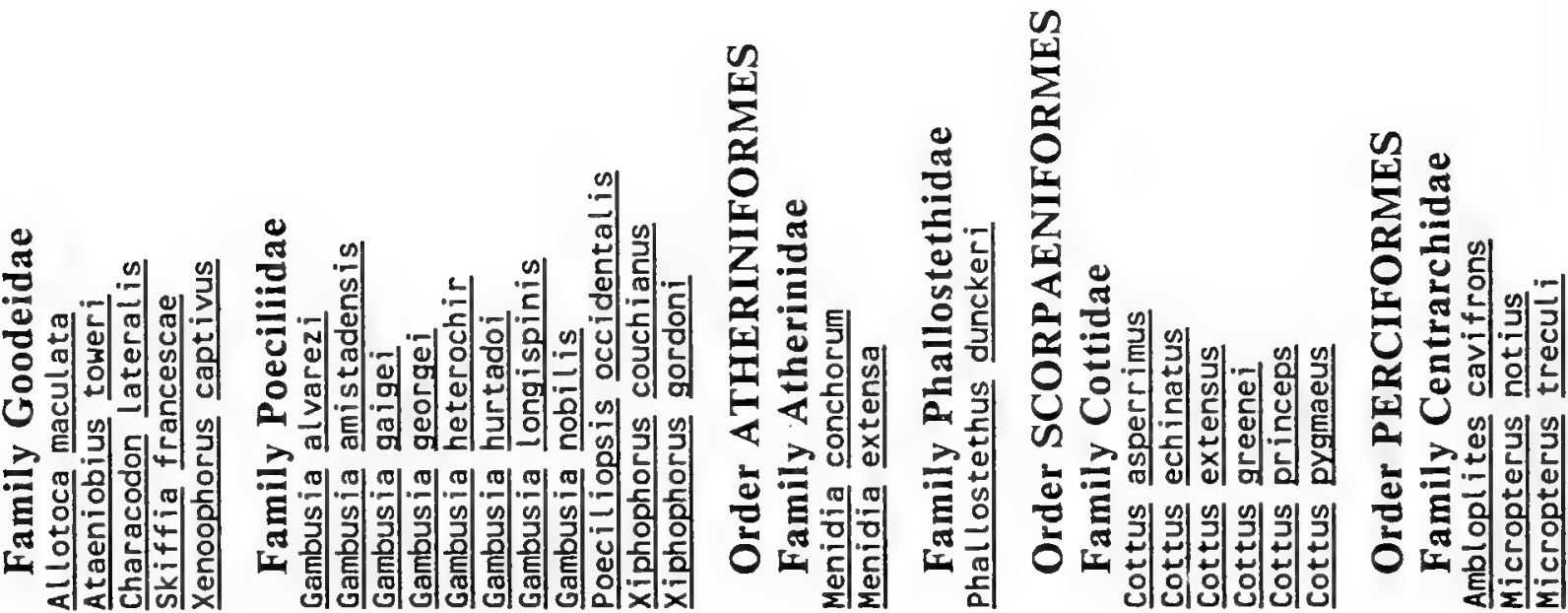


\section{ذ)}

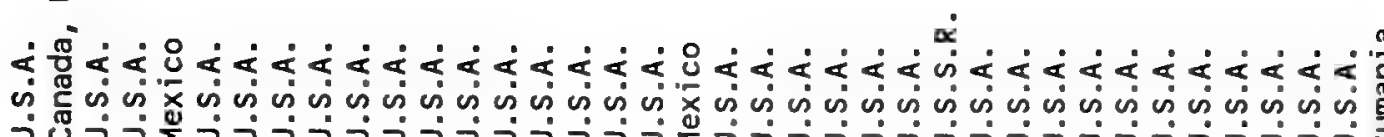

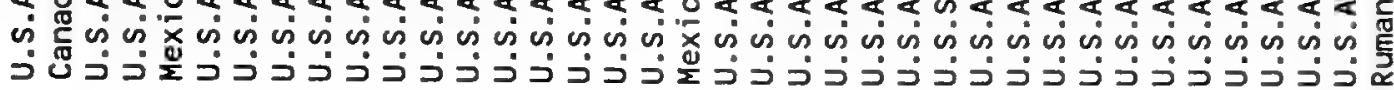

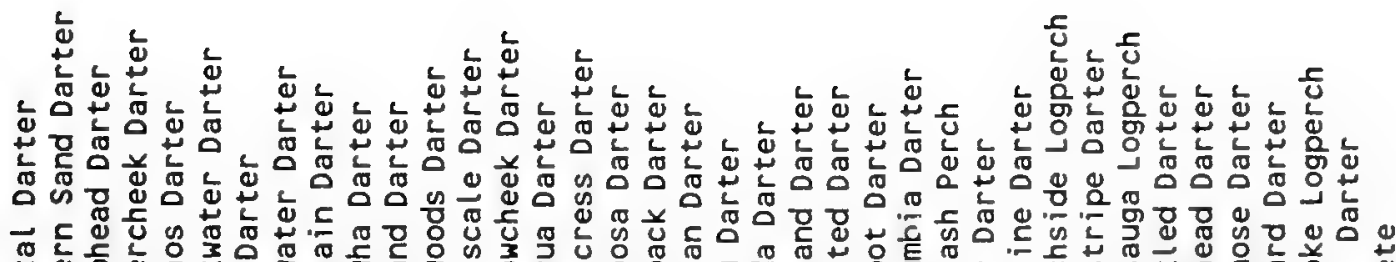

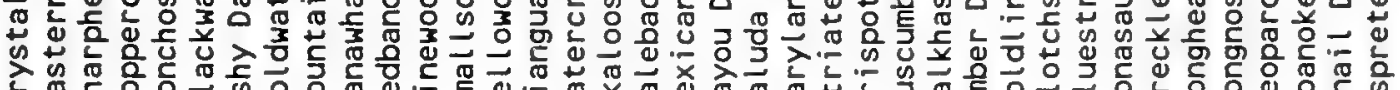

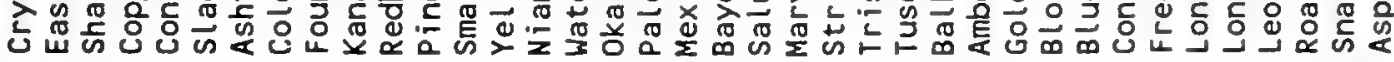

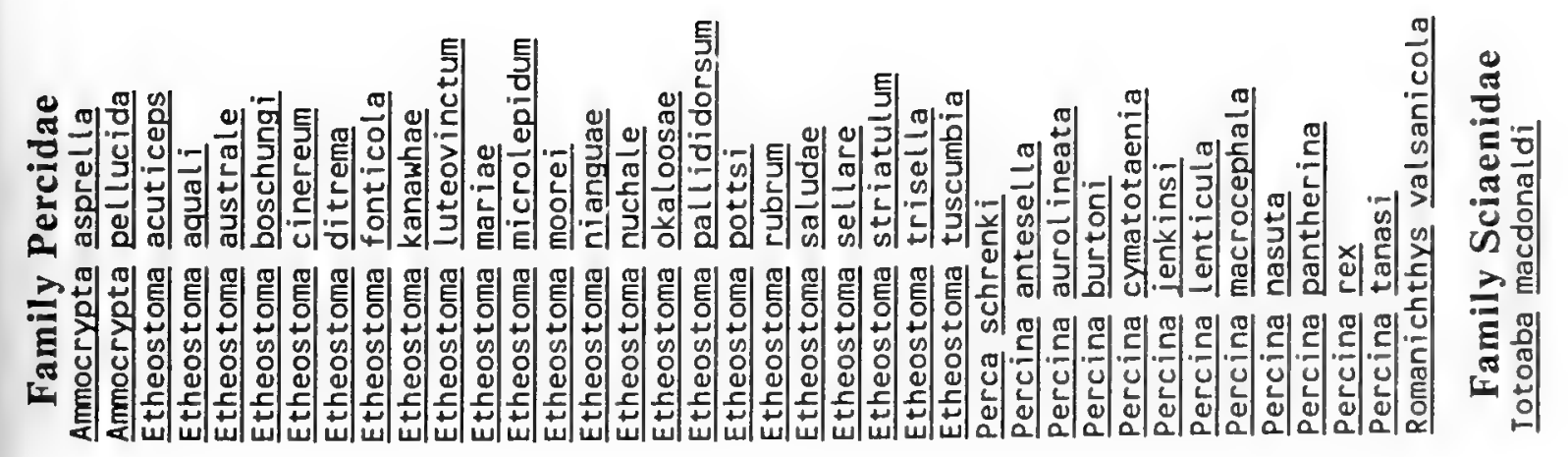




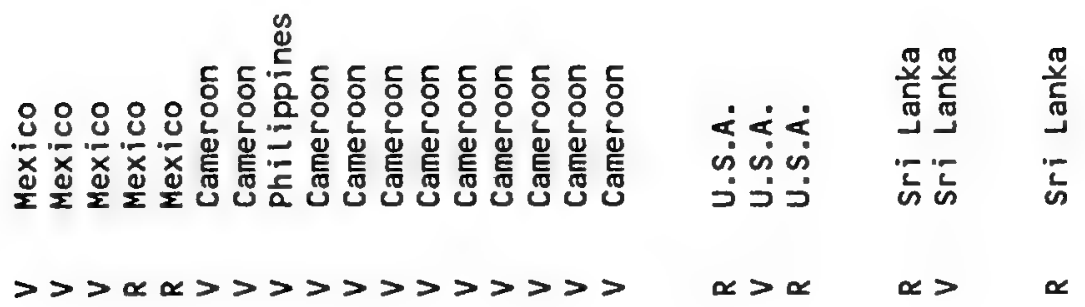

0
0
0
0
0
0
0
0

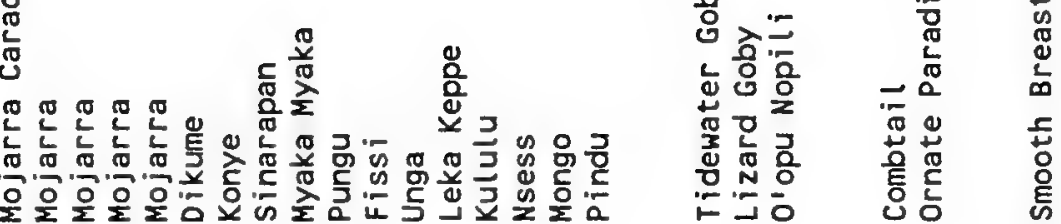

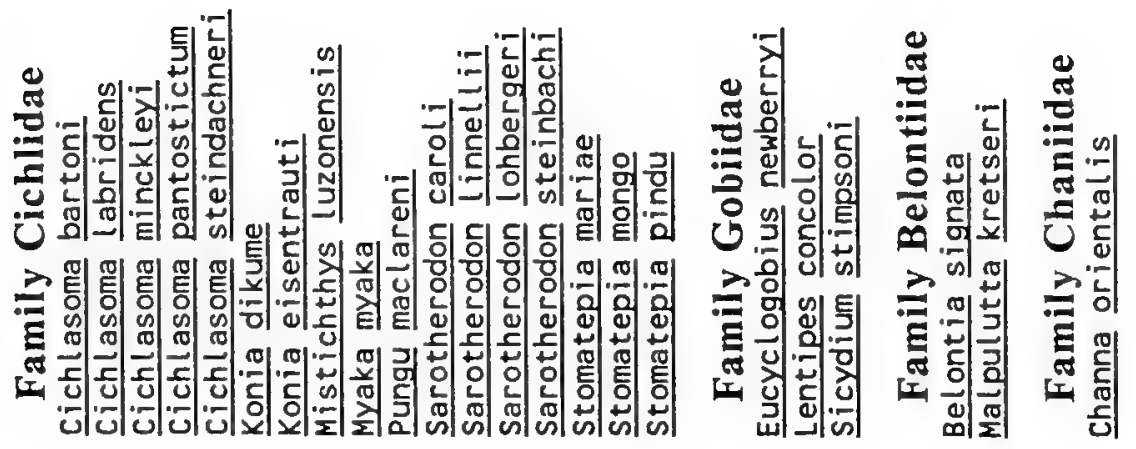




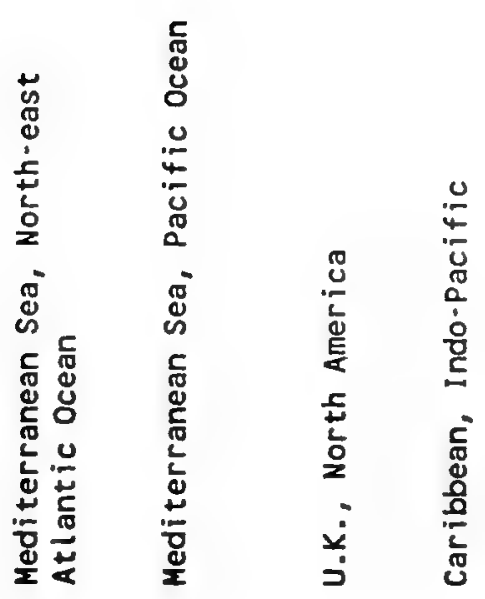

ํㅗㄹ

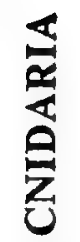

七 $>5$

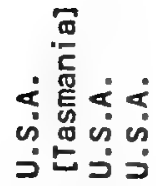

س
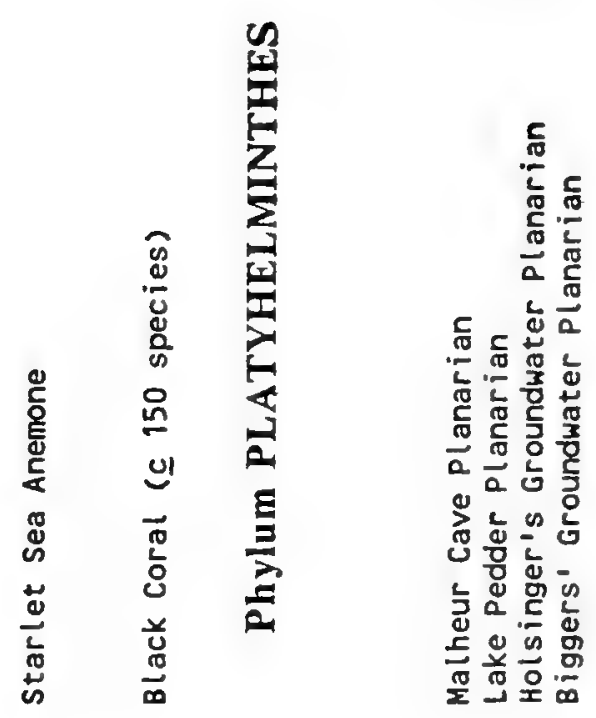

承

昰

$\frac{\widehat{g}}{\bar{g}}$

总

응

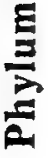

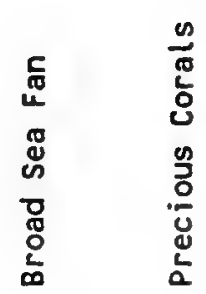

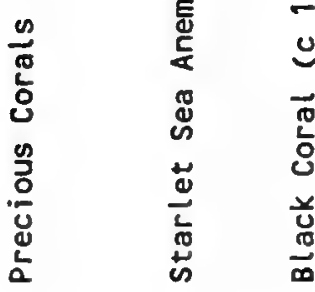

c $\frac{5}{0}$

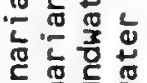

동 홍

这电

잉

远

줄오요

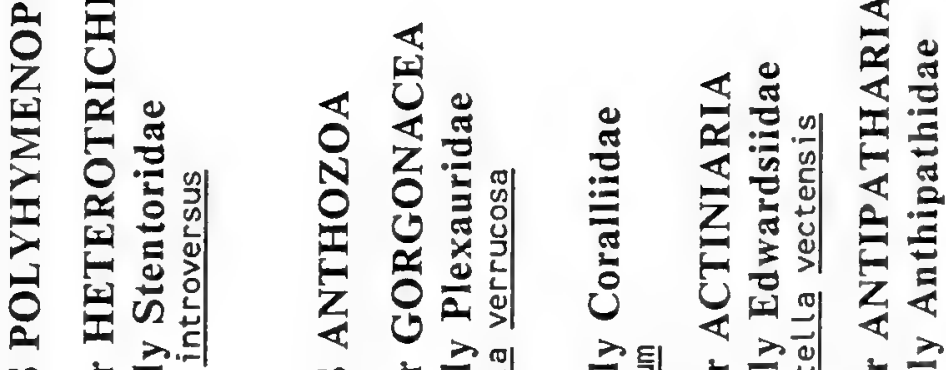

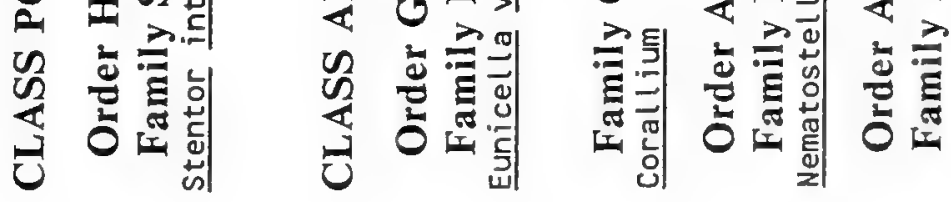

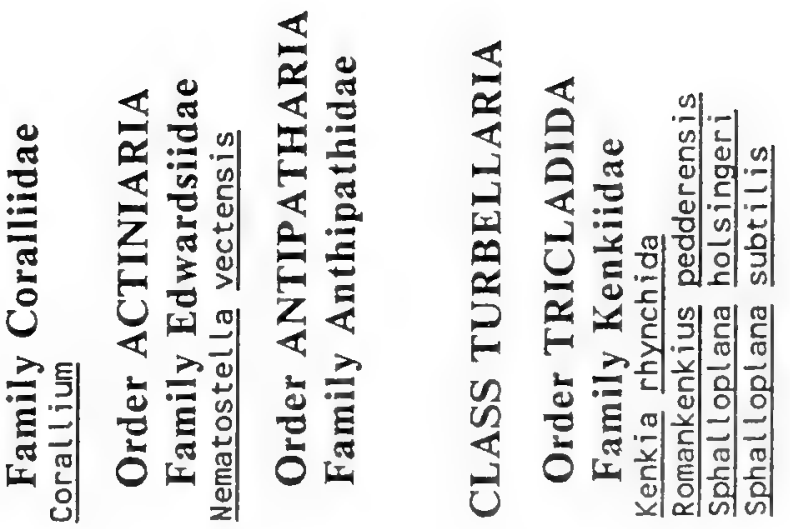



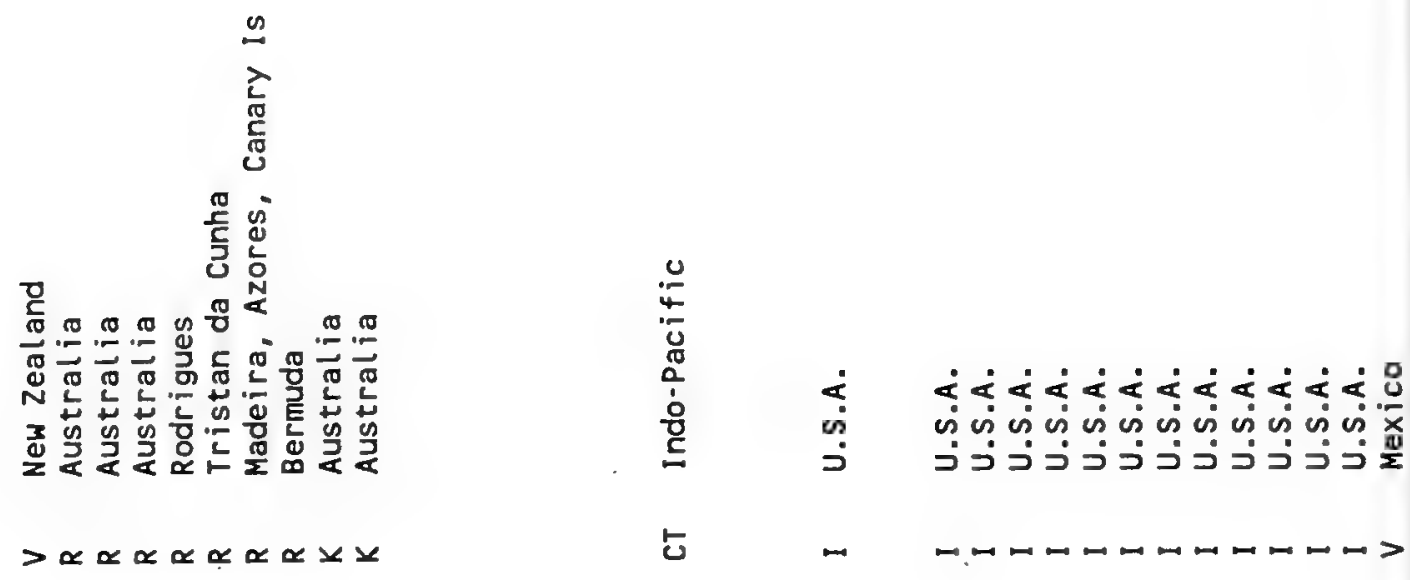

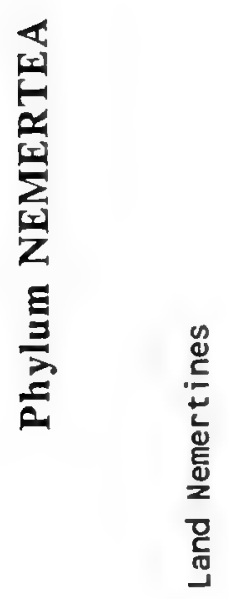

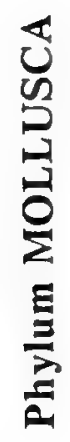
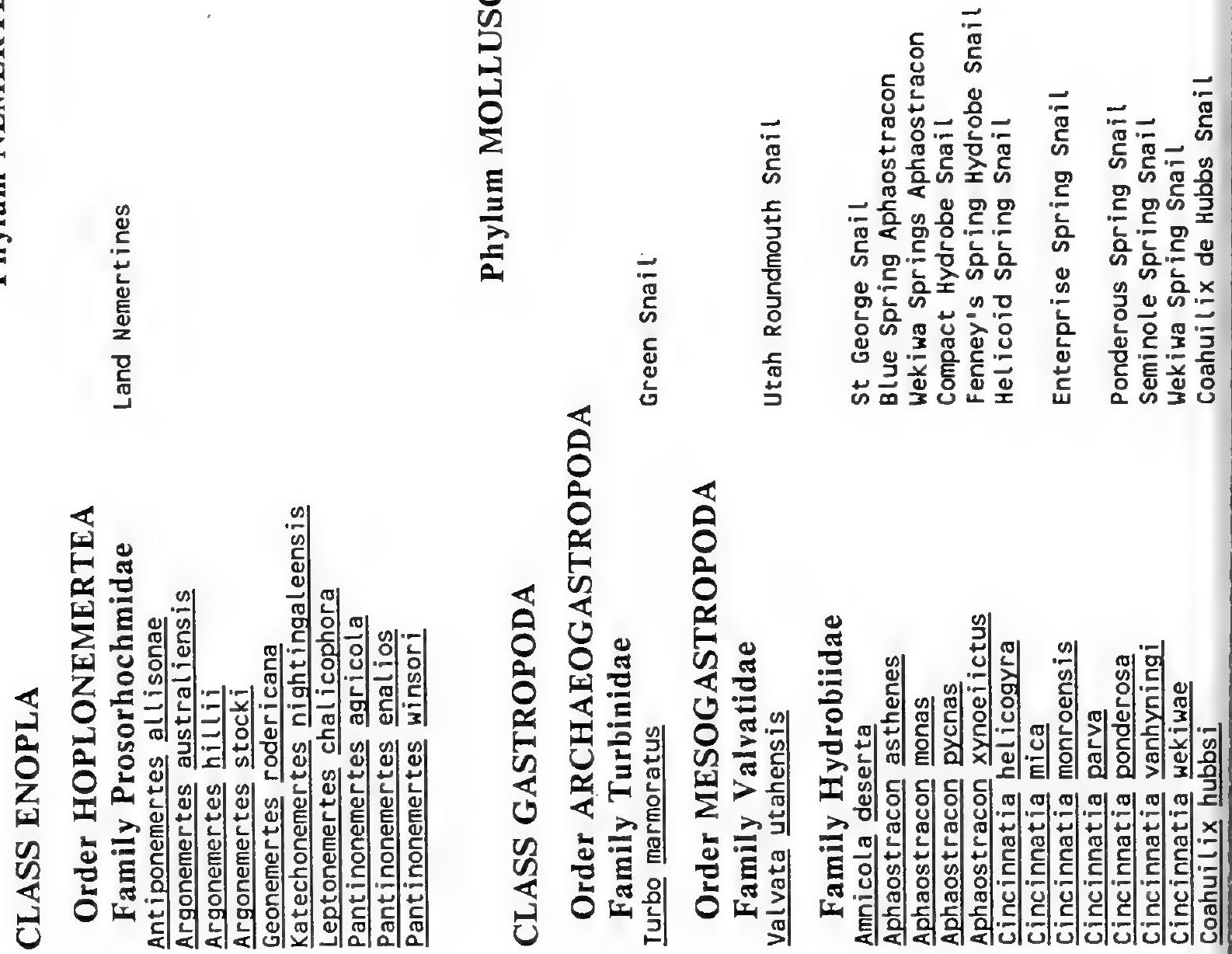


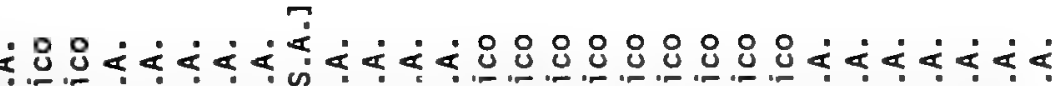

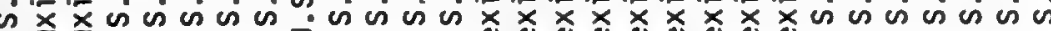
ن

$\dot{4} \dot{x}$

央

ว

$<\dot{<} \dot{<} \dot{<} \dot{<} \dot{<}$

is os is is in is is

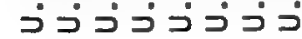
艾

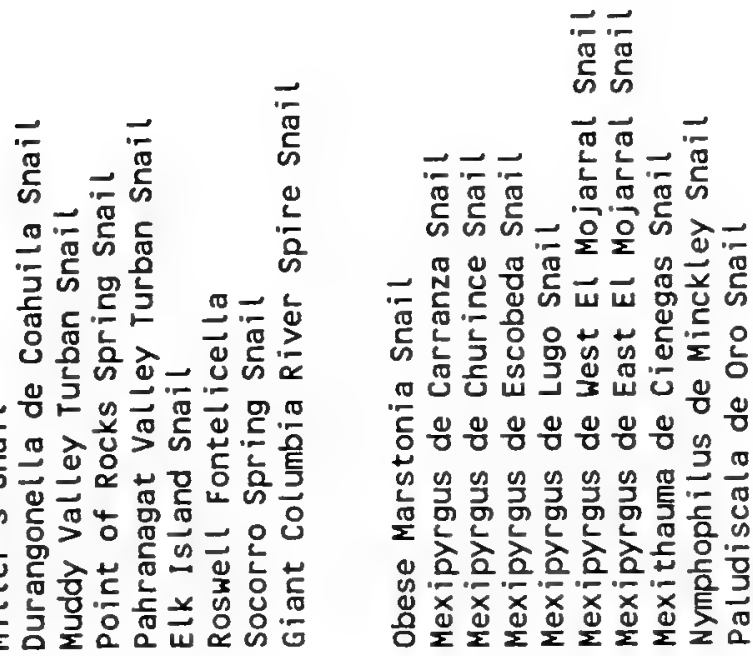

"ृ<smiles>[Li][Ca]</smiles>
$-\frac{5}{n}$ $=\pi$ 的商 is $\sum^{2}$.

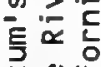
苗芒 $\stackrel{0}{=}$

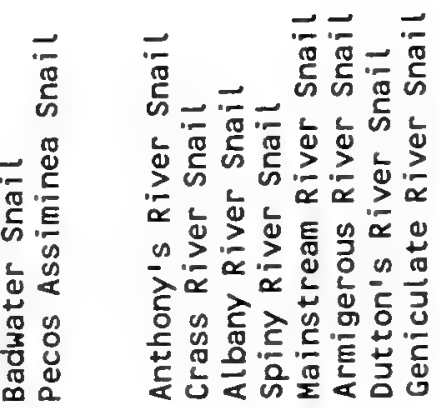
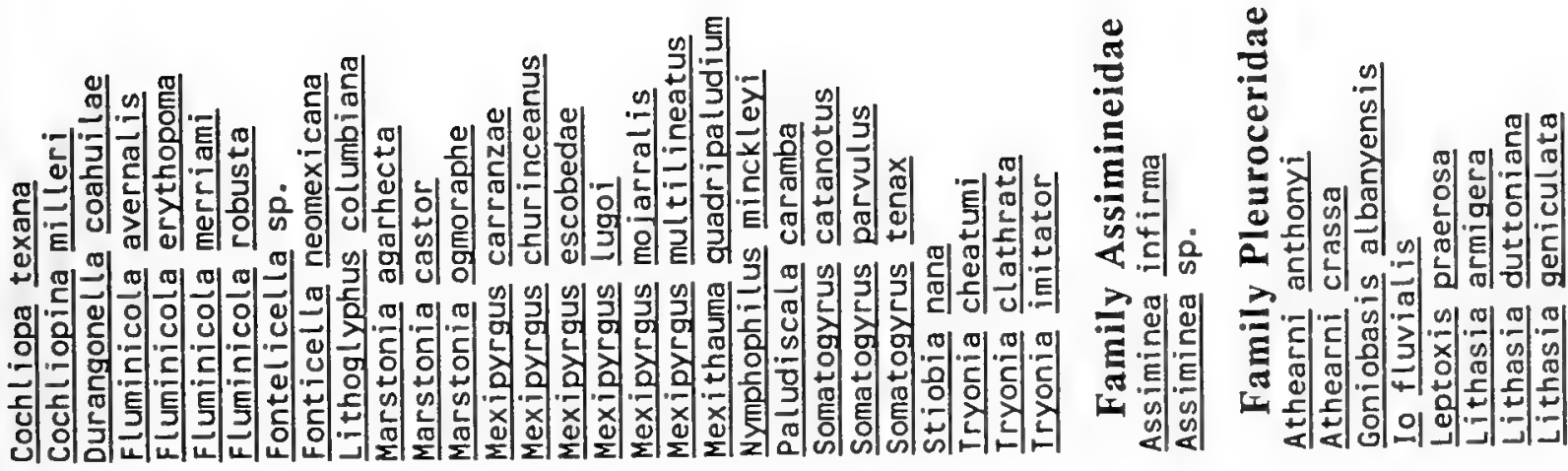


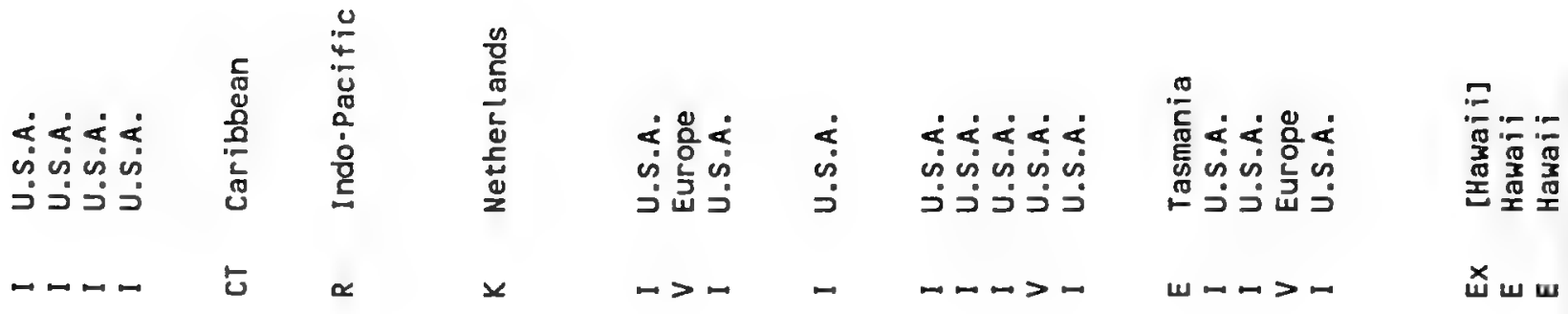

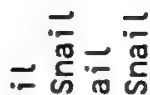

क के क

品乎出

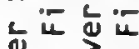

$\sum \frac{1}{\alpha} \pm$

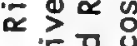

co $\alpha$ 过

잔 은
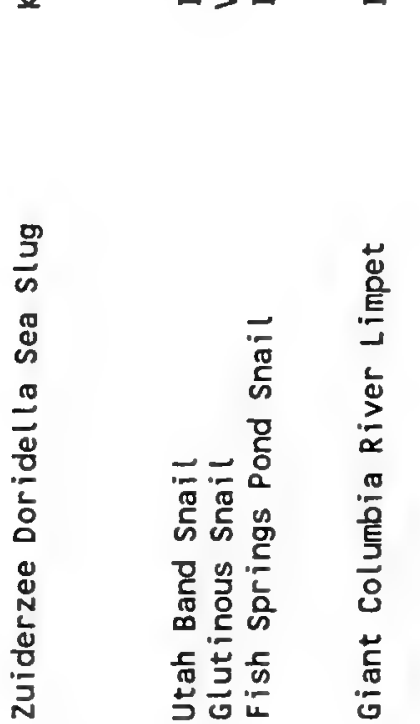

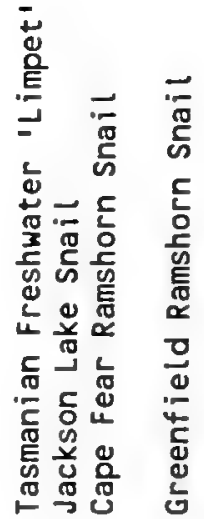

$\stackrel{\infty}{\stackrel{\infty}{\rightleftarrows}}$

๑

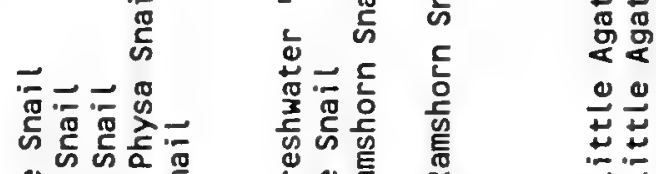

总

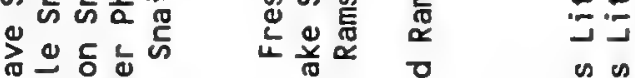

\& \&

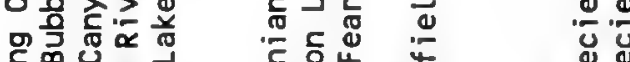

这这

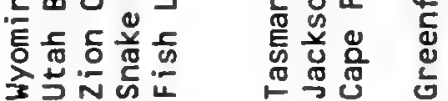

Na

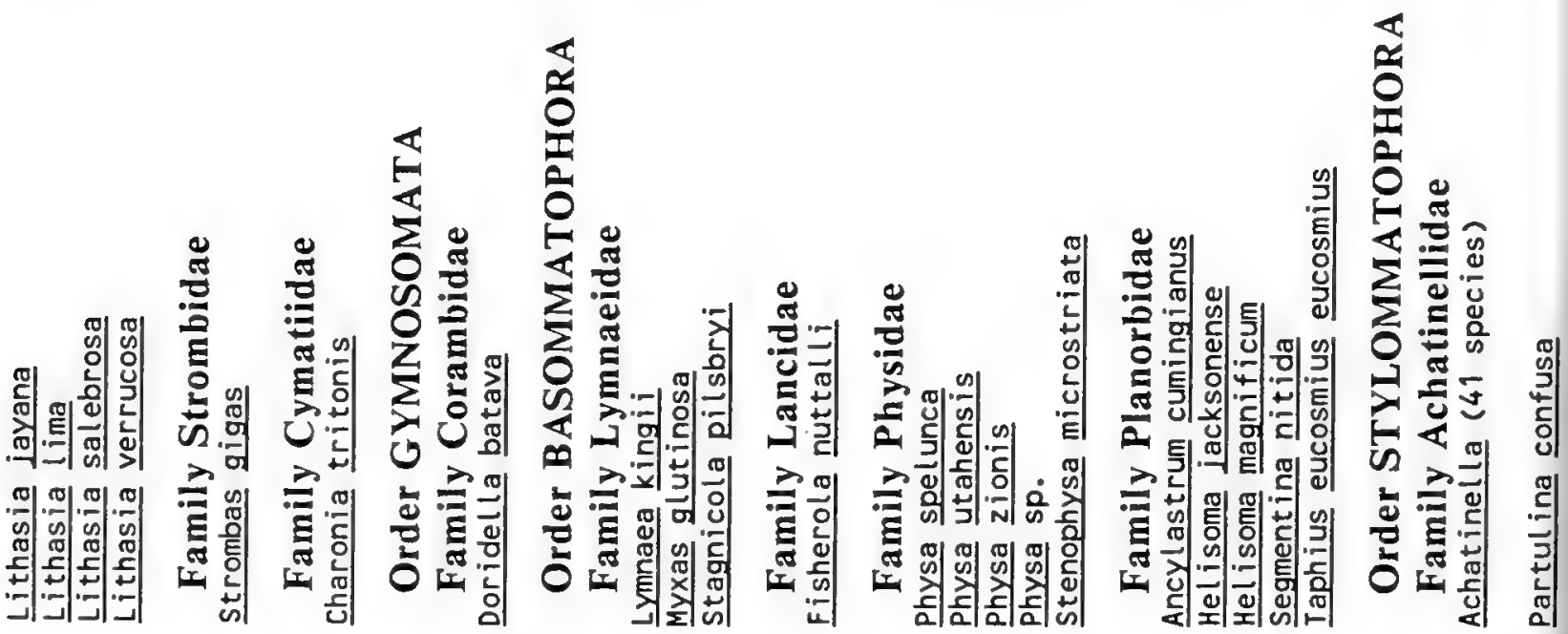




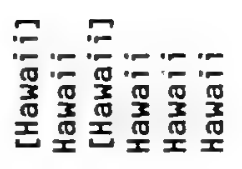

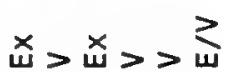

ֻั:

心舟

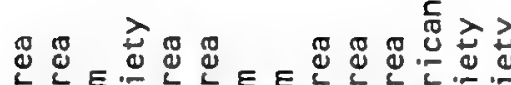

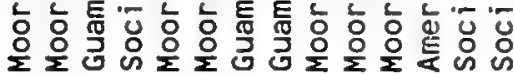
崌

뚜
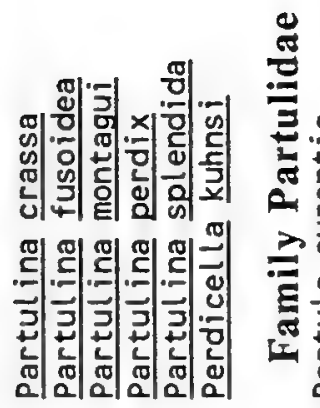

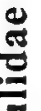




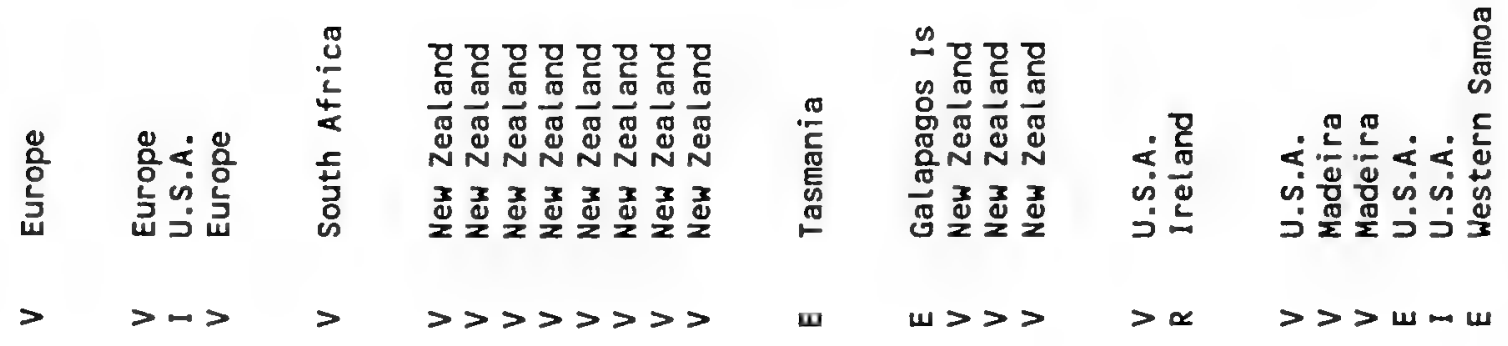

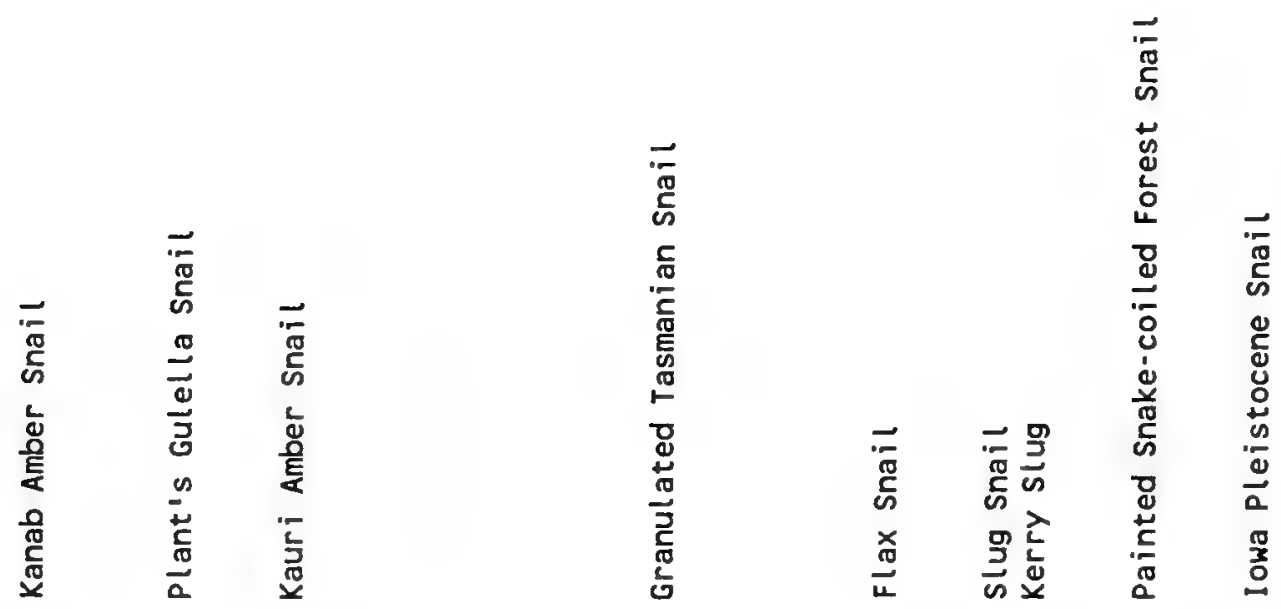

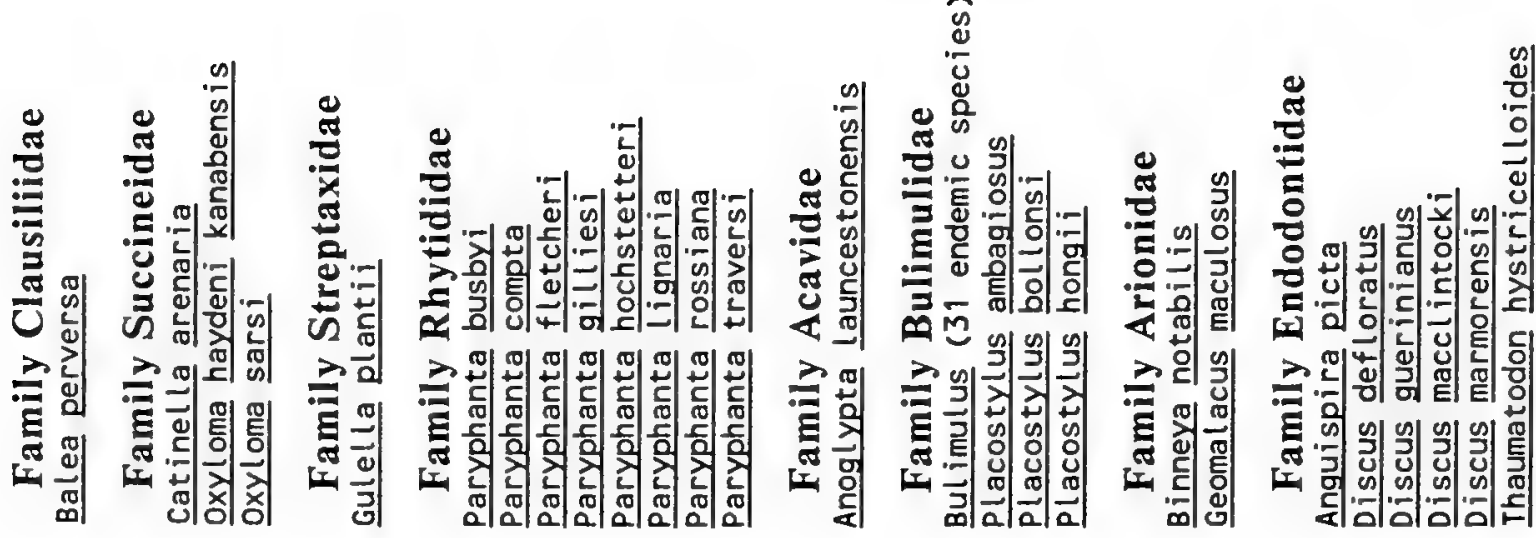



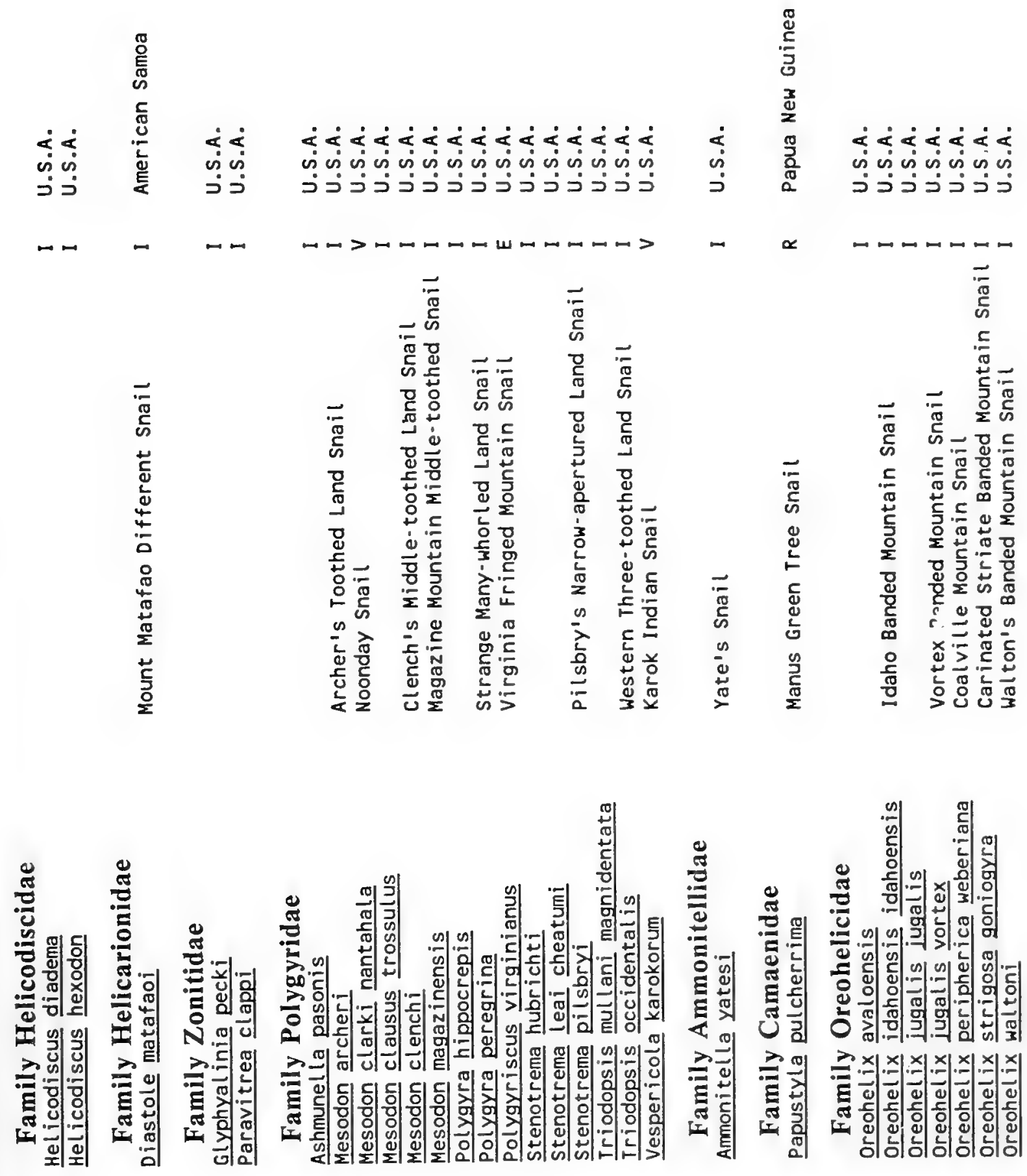


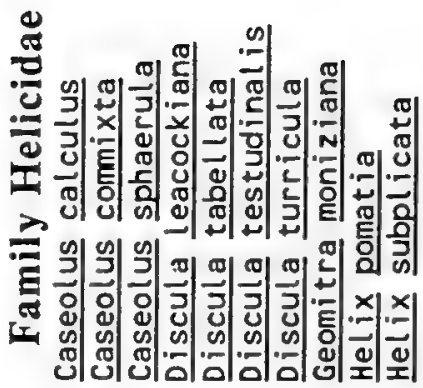



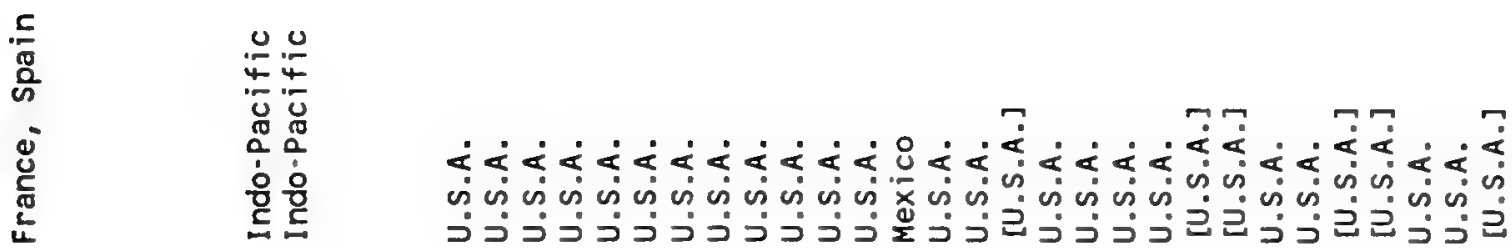

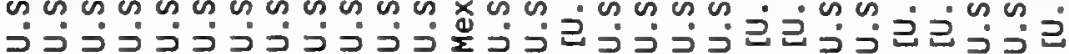
七 $\tilde{x} \times \ldots \tilde{x}$

文这

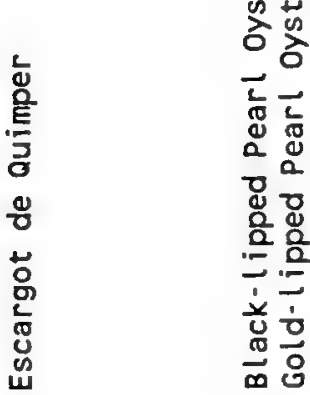
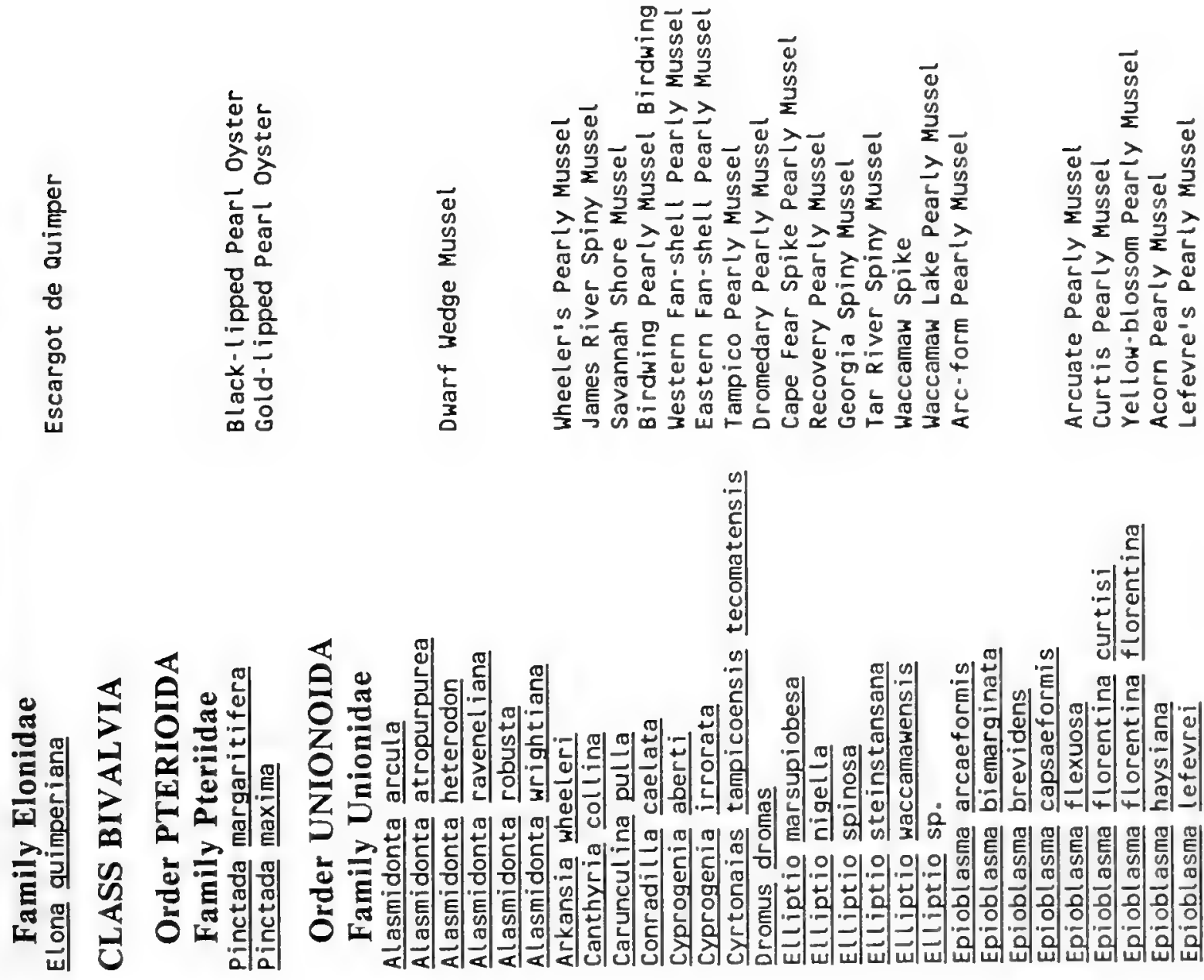


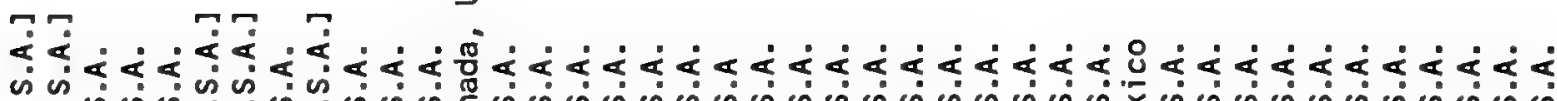

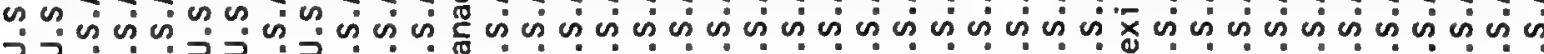

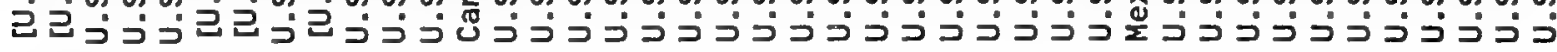
这
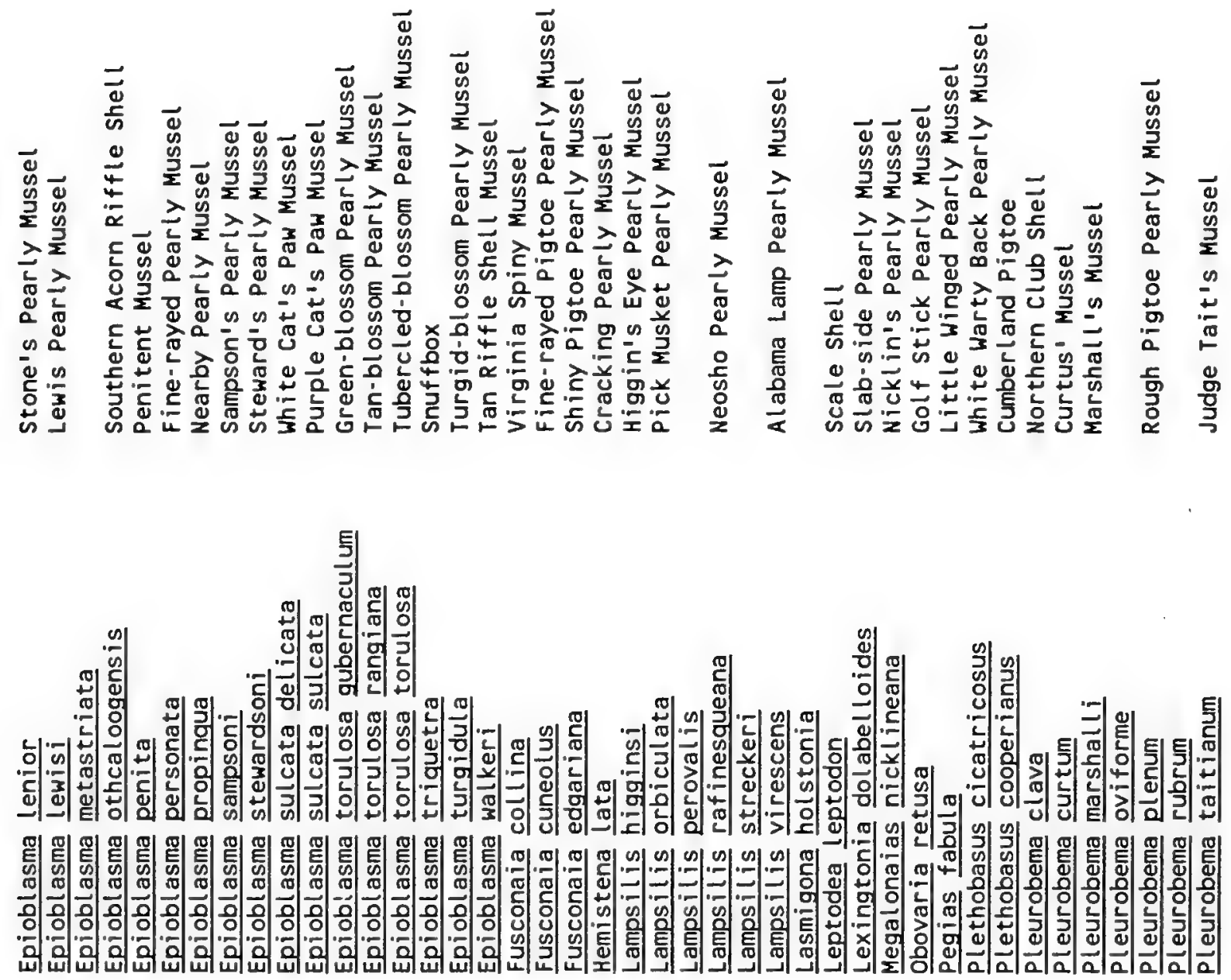


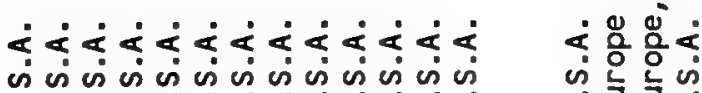
๒

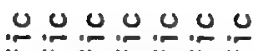

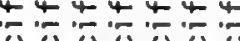
u y u y $\begin{array}{ll} & \\ 0 & 0\end{array}$
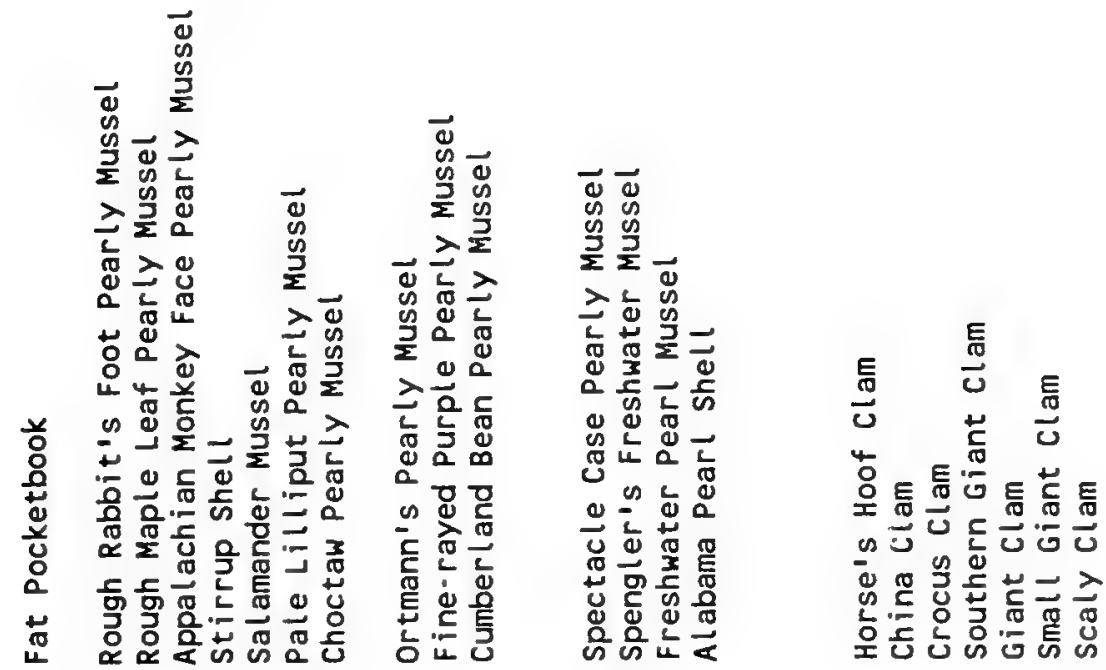

夏

$E$
$\frac{1}{3}$
$\frac{0}{3}$
$\frac{0}{0}$
$\frac{0}{0}$
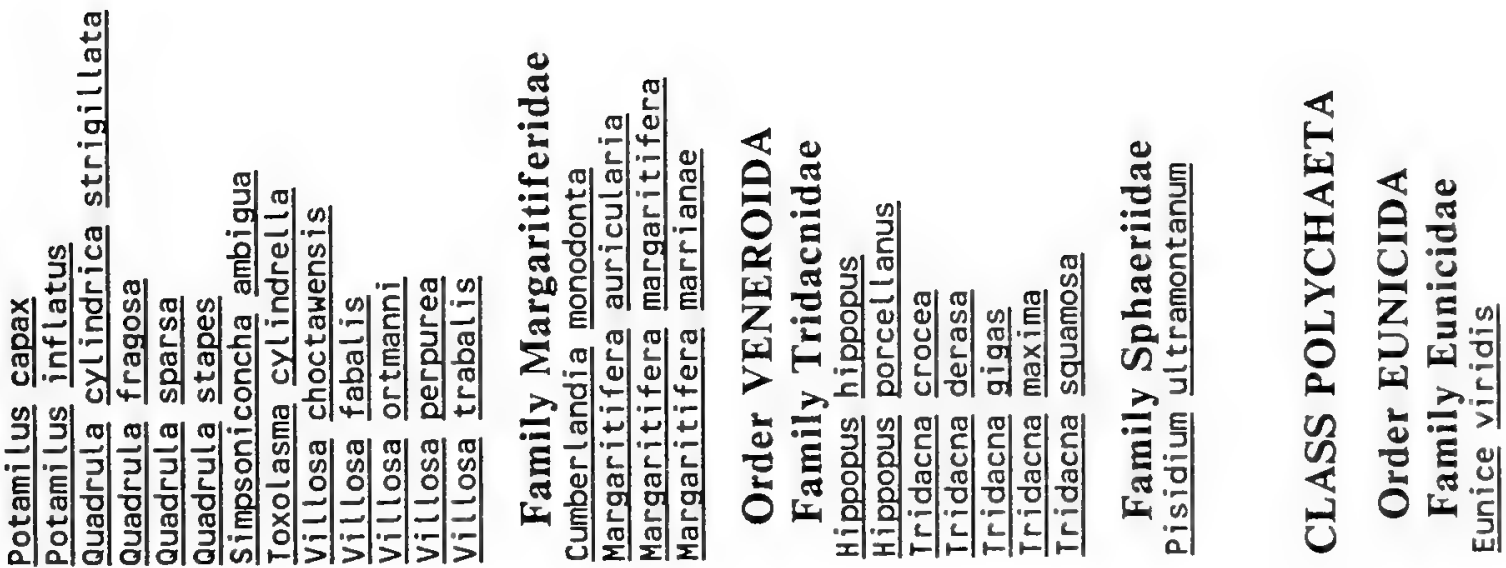
告

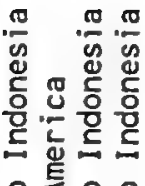

운운웅

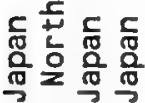

. 范.

结藏造题

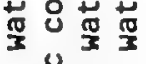

$\pi \frac{\pi}{\pi}$

出的易

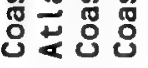

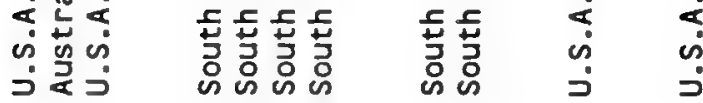

$x x x$

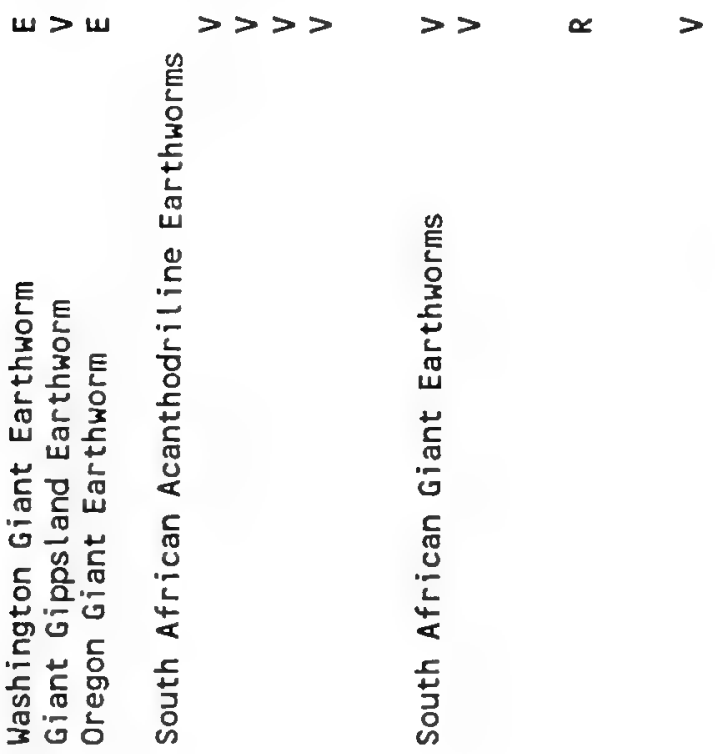

(1)

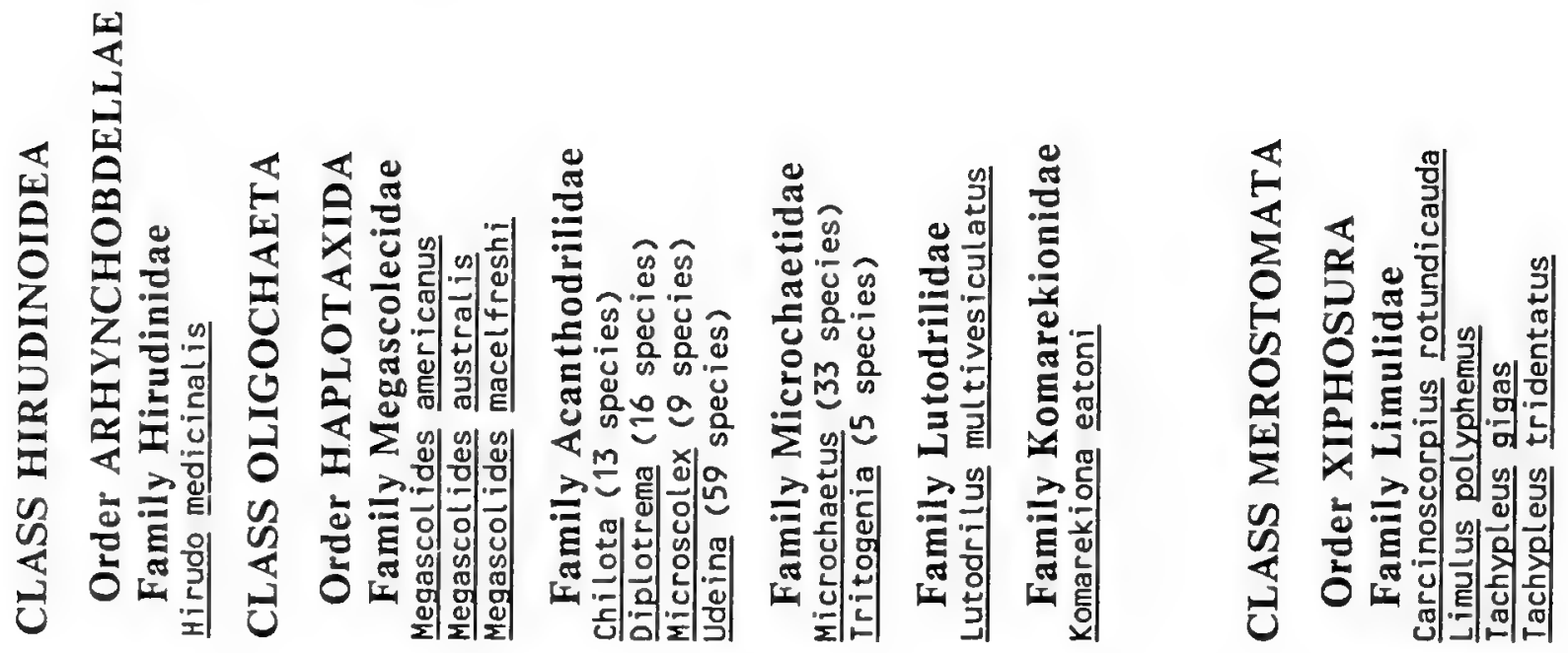




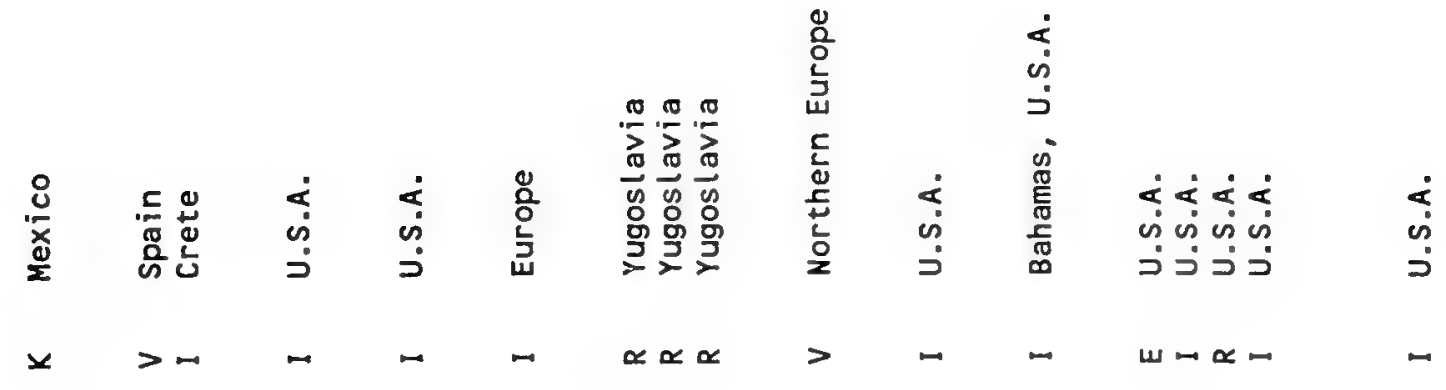

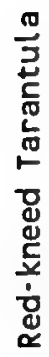

这密

क⿺辶冋

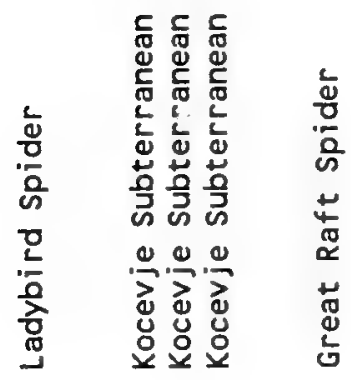

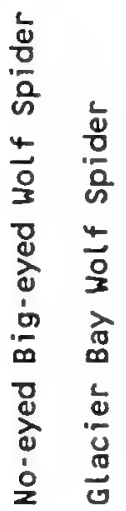

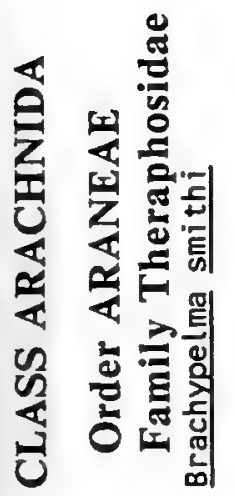

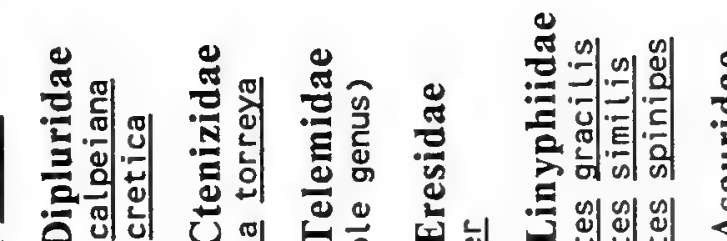

則

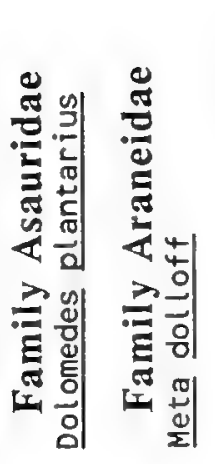

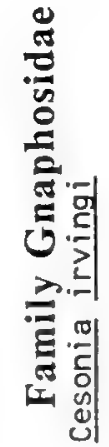

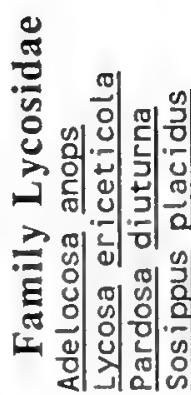

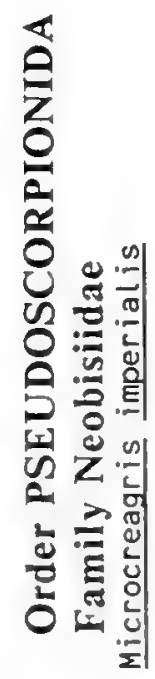



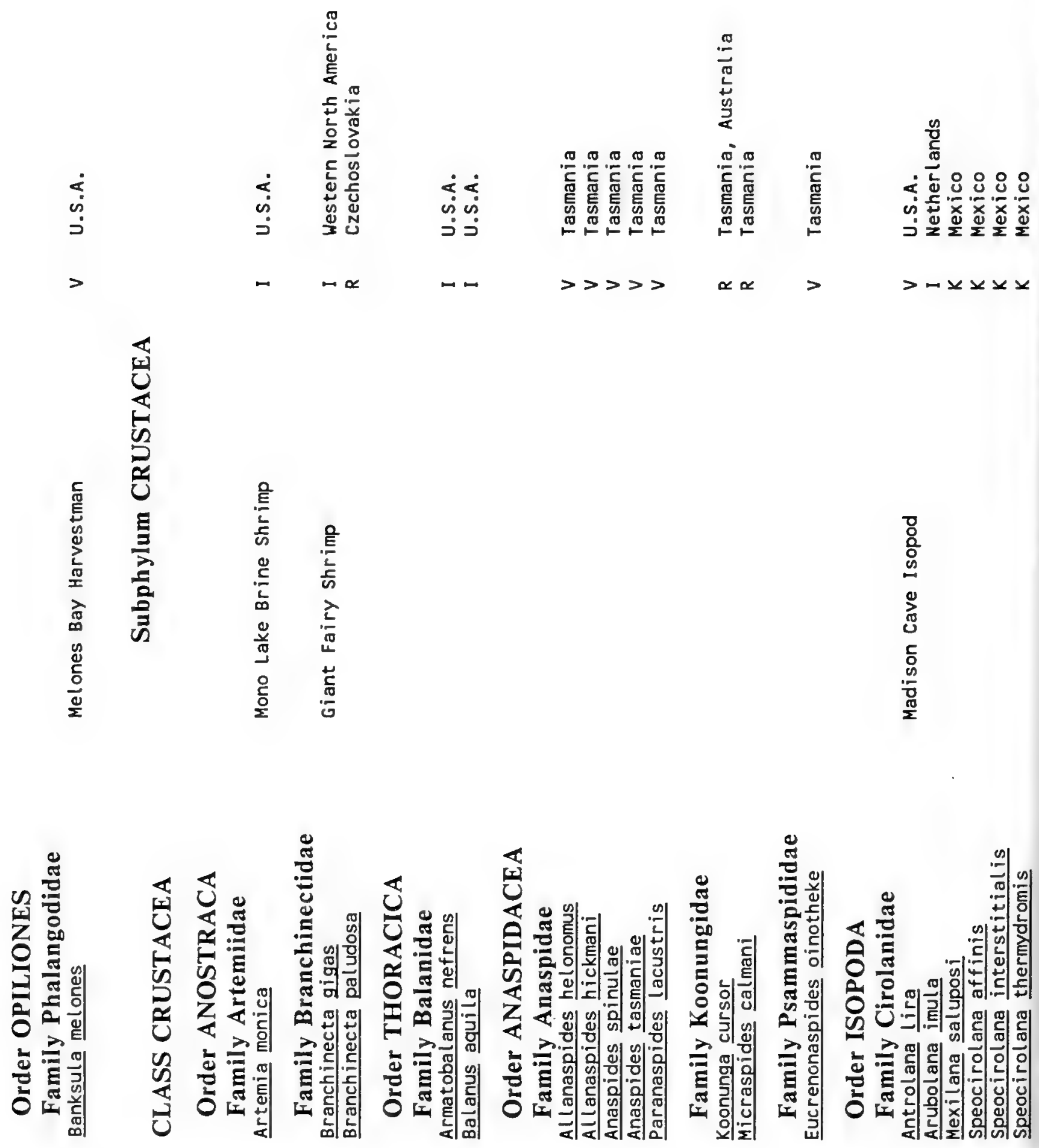


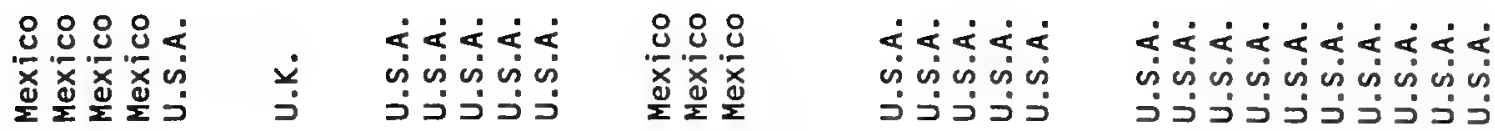

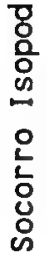

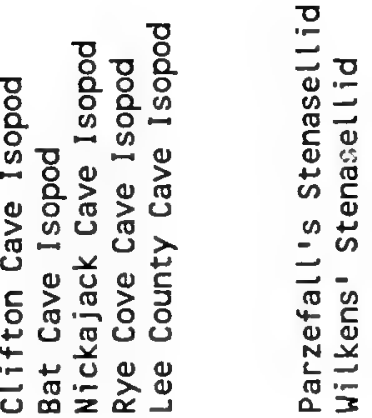
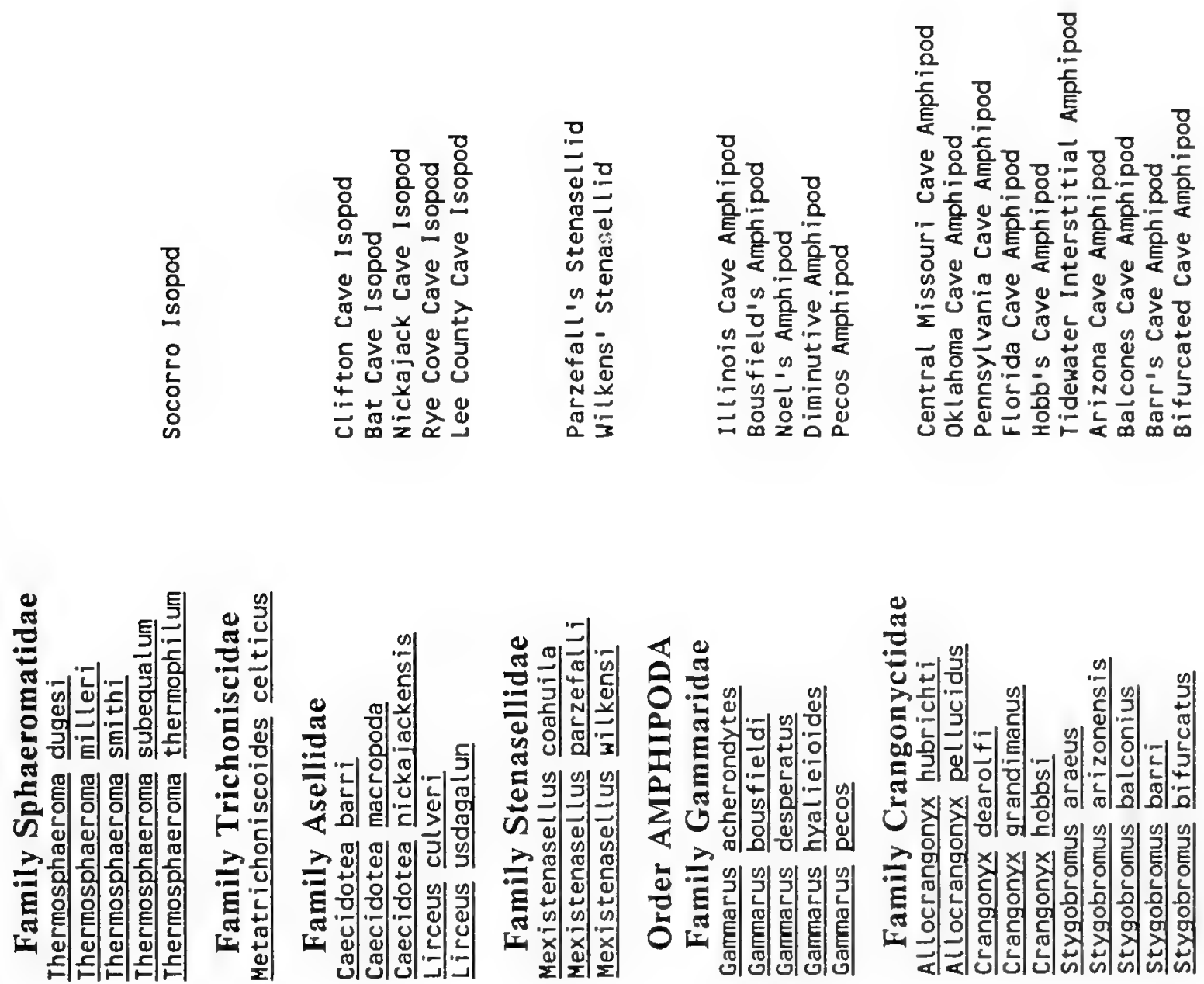


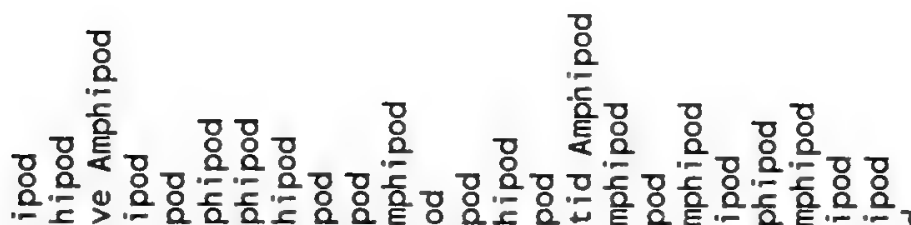

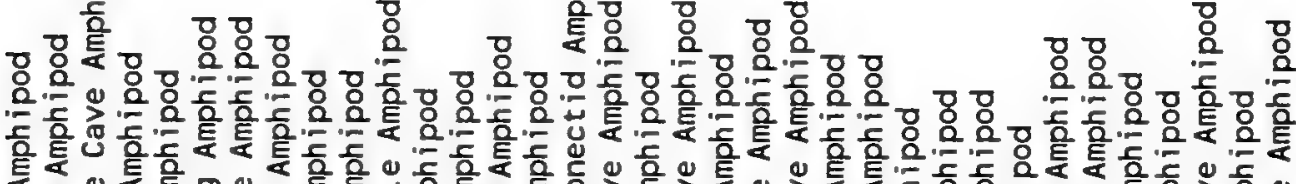

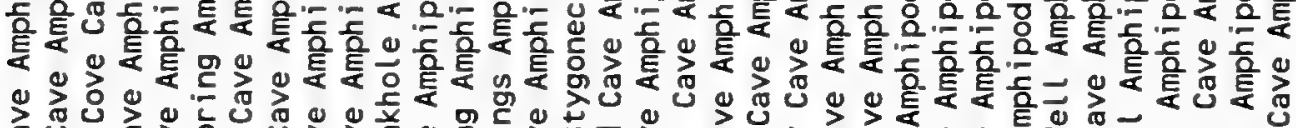

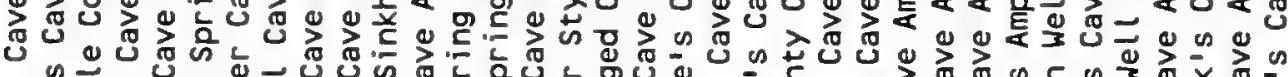
n

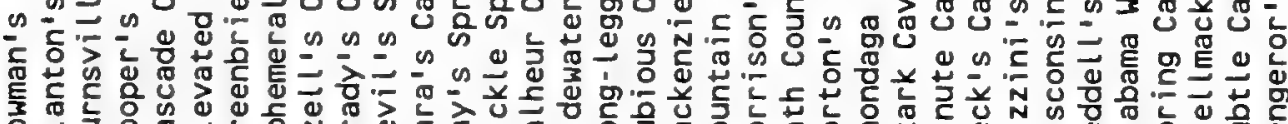

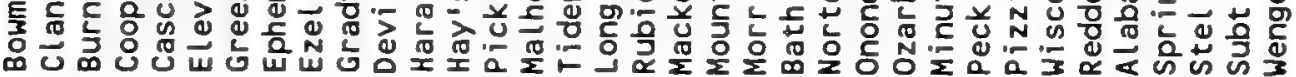

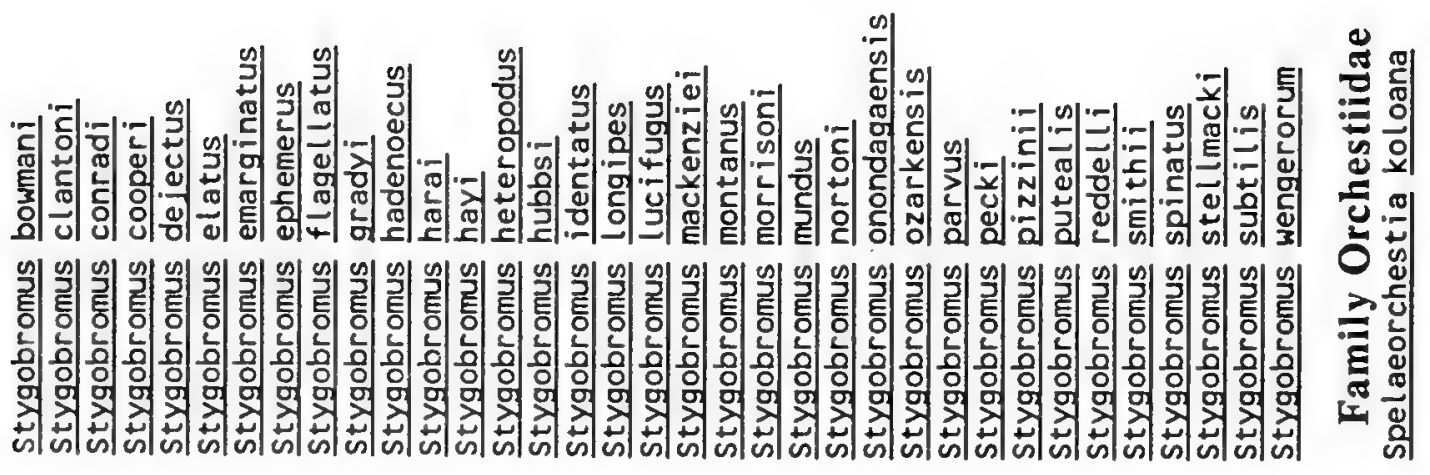


$\frac{2}{\frac{2}{2}}$

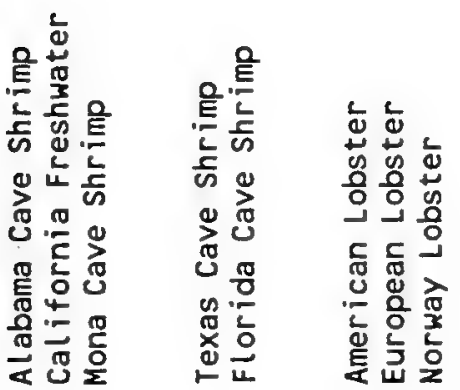

\section{$\frac{\sqrt{n}}{\frac{1}{4}}$}

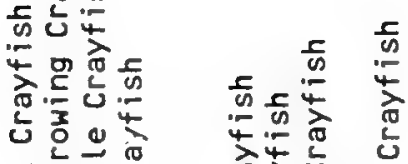

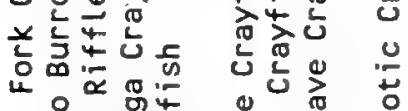

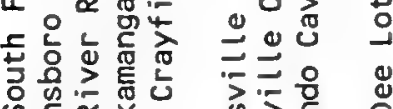

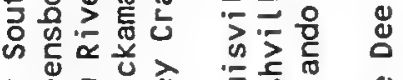

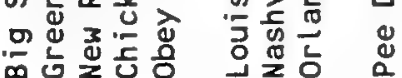

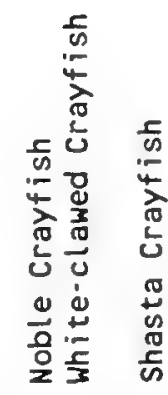

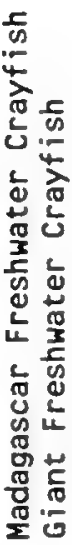




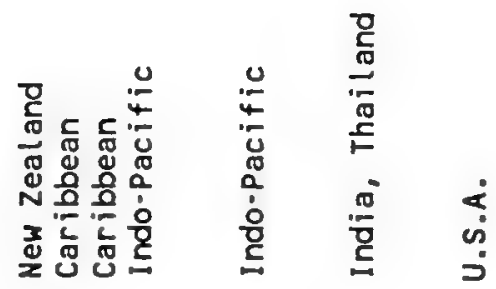

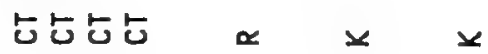

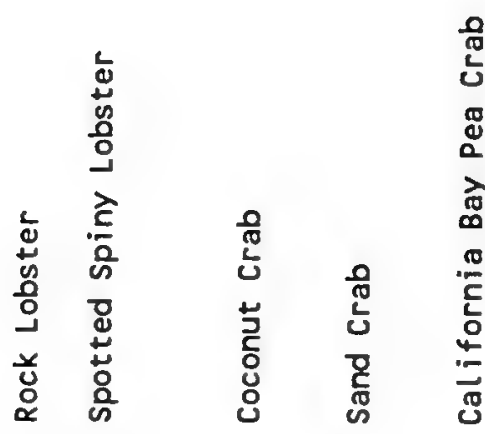

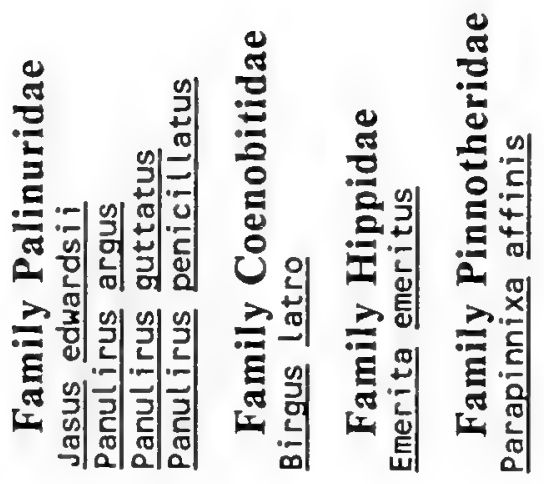

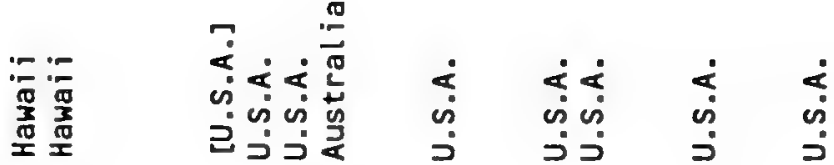

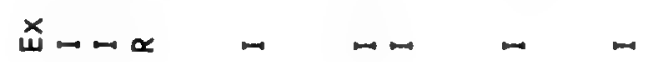

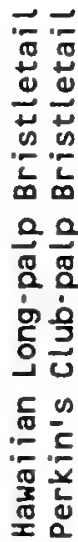

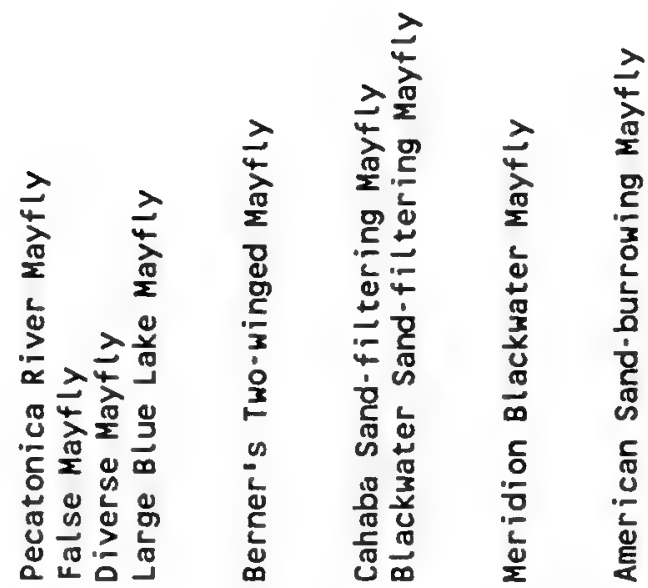

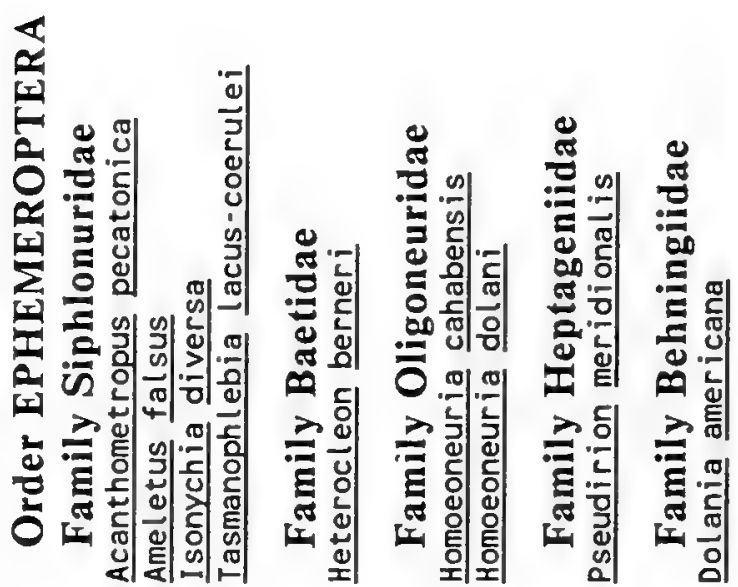




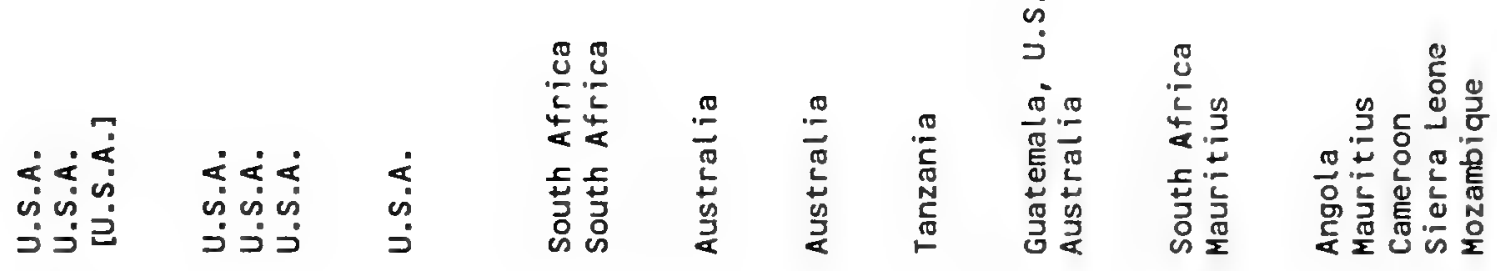
ーーய ー -

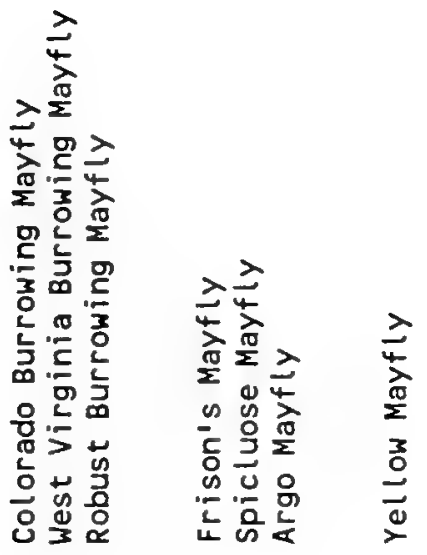

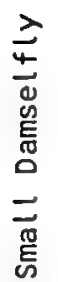

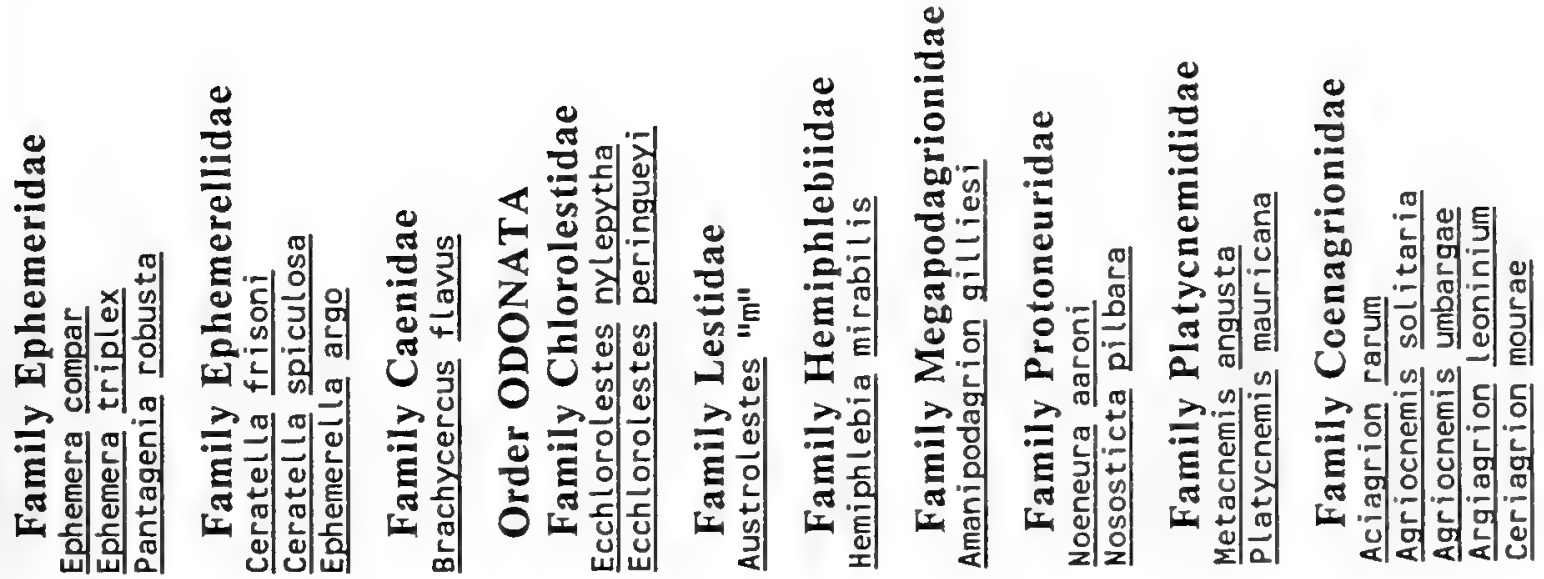




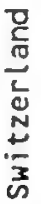

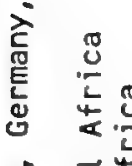

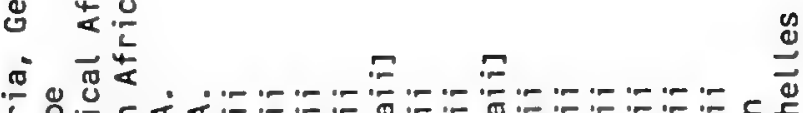

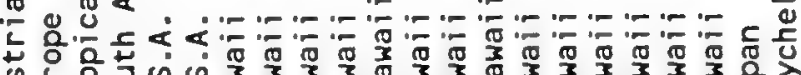

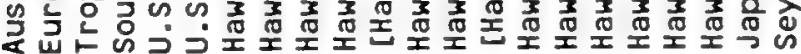

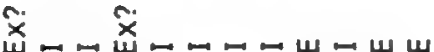
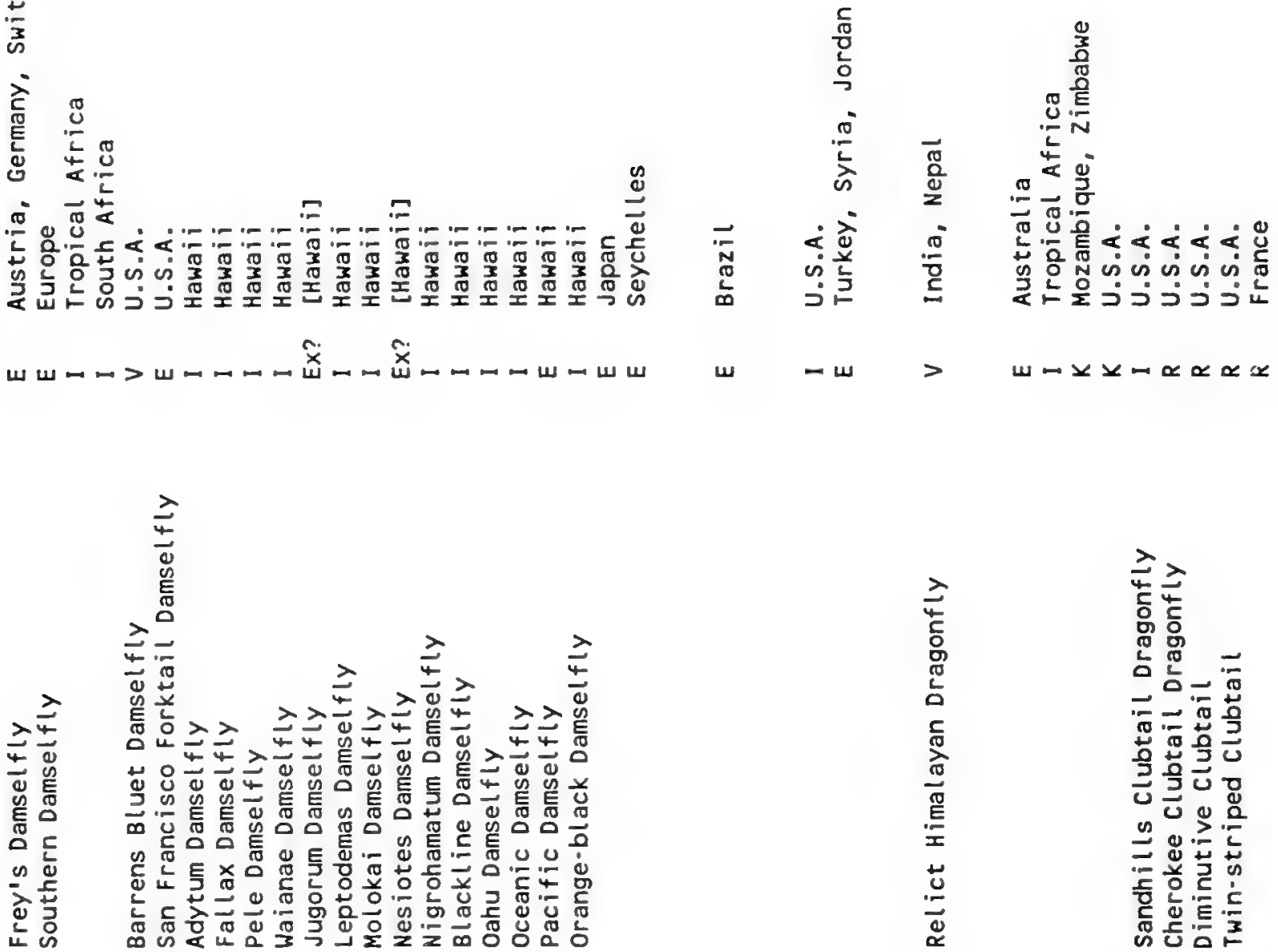

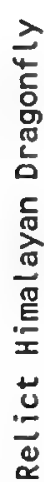
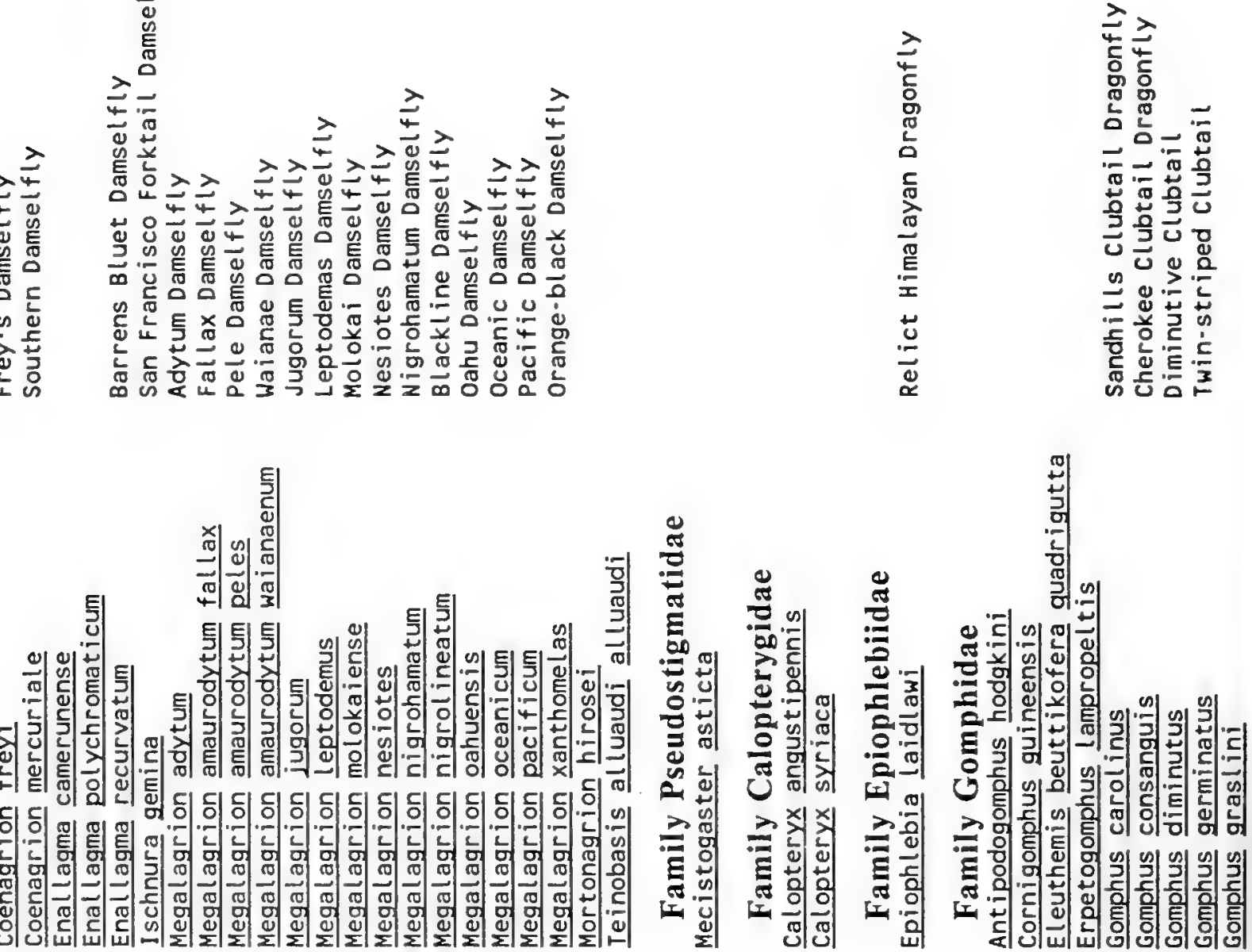


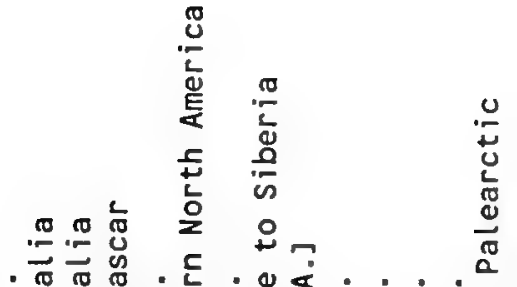

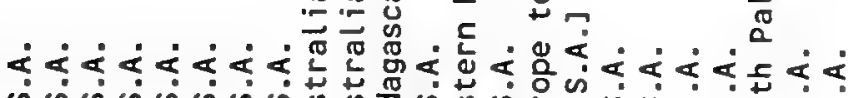

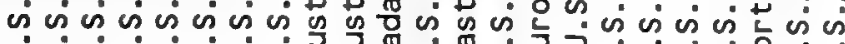

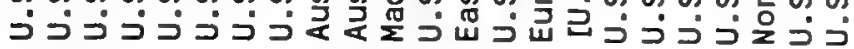
x.

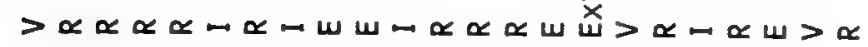

$\geq 2$

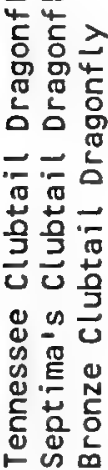

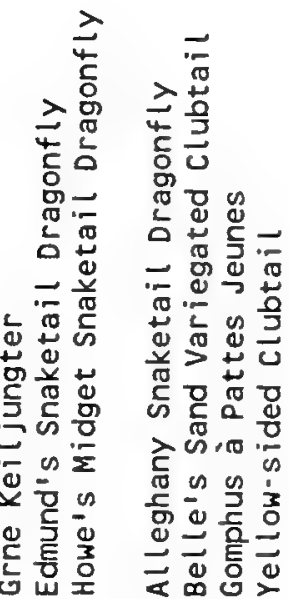

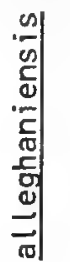

क्षी

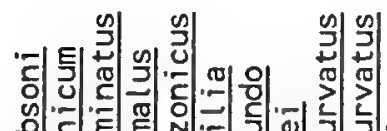

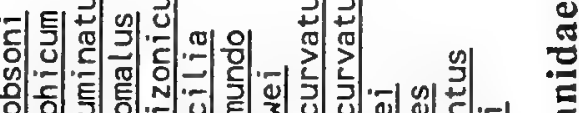

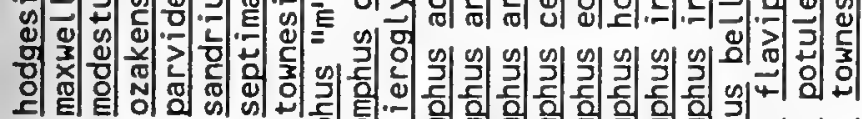

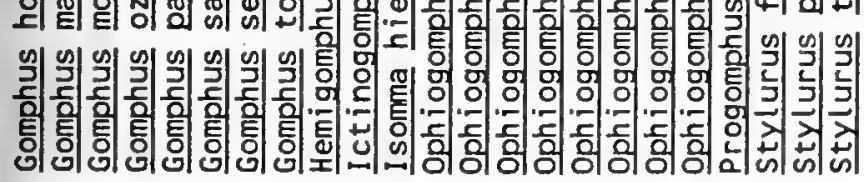

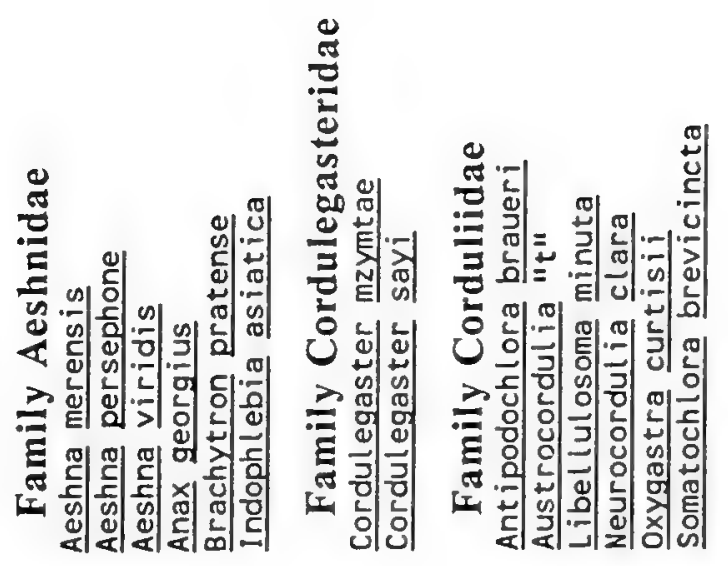

童皆

눈

舟

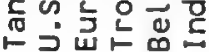

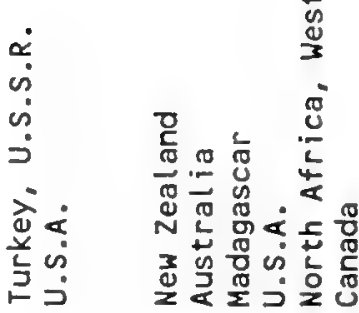

$\propto \propto \omega \sim>>$

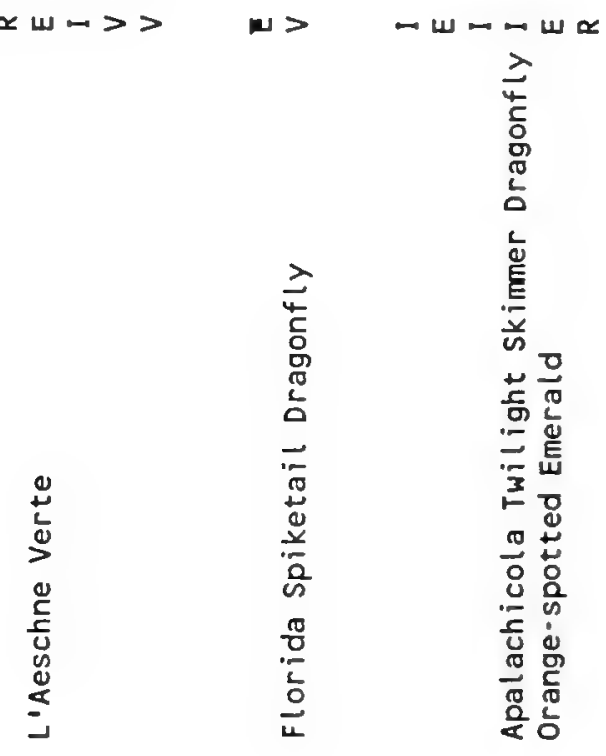




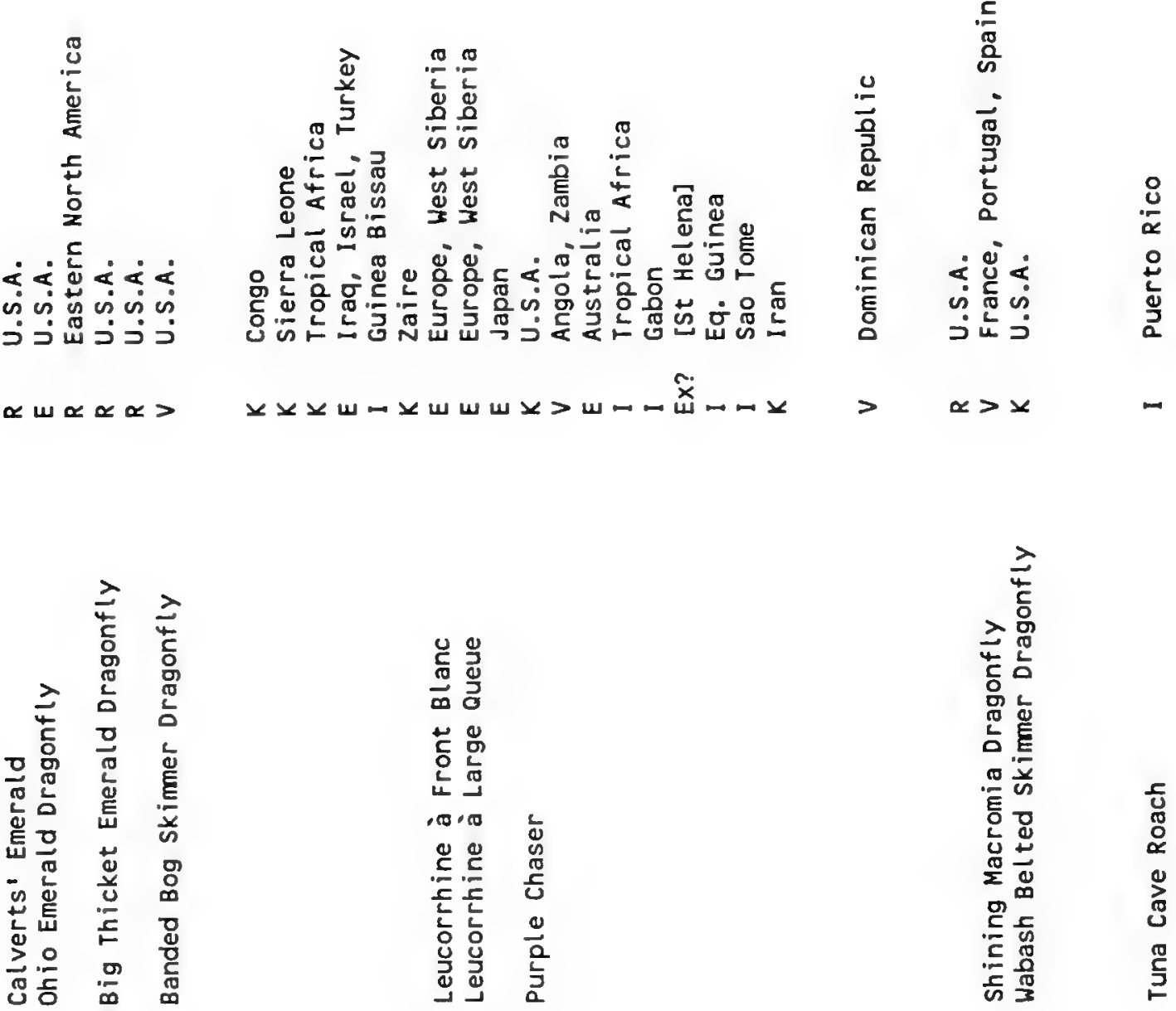

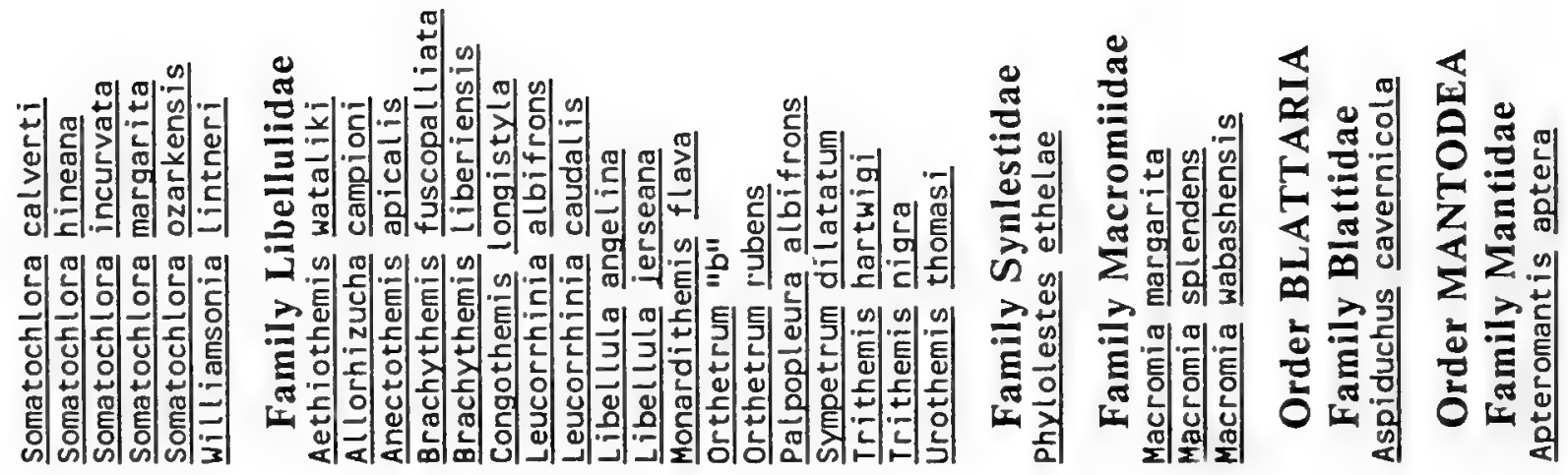




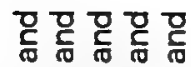

응

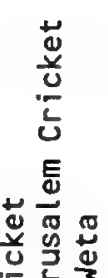
苍专苟

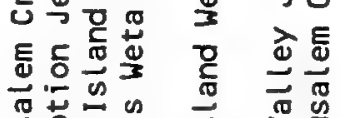

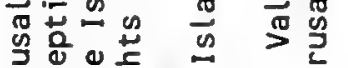

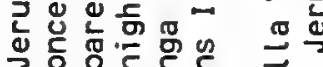

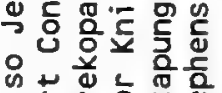

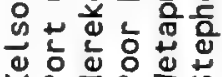

\section{范 \\ $\frac{\ddot{0}}{\frac{1}{2}}$ \\ 曾 \\ 造}

음

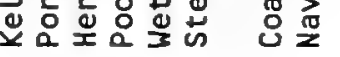

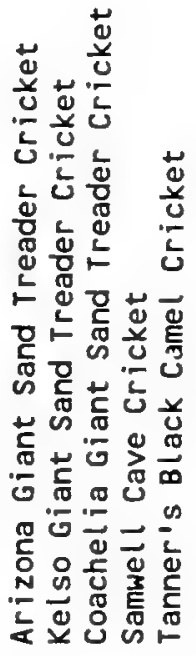

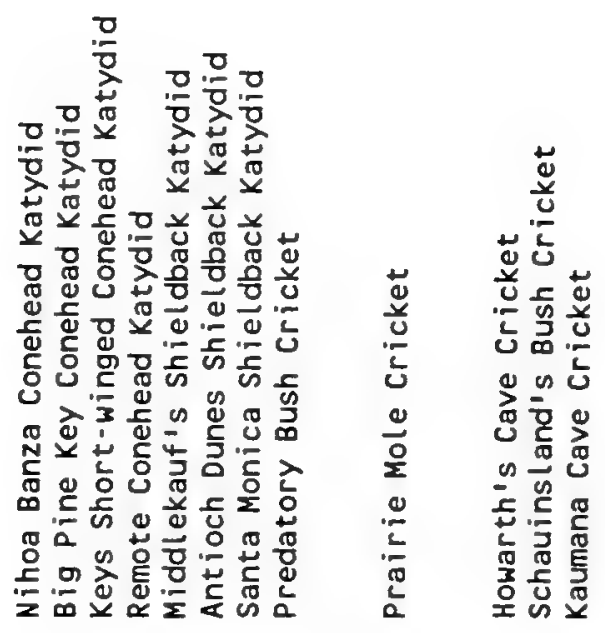
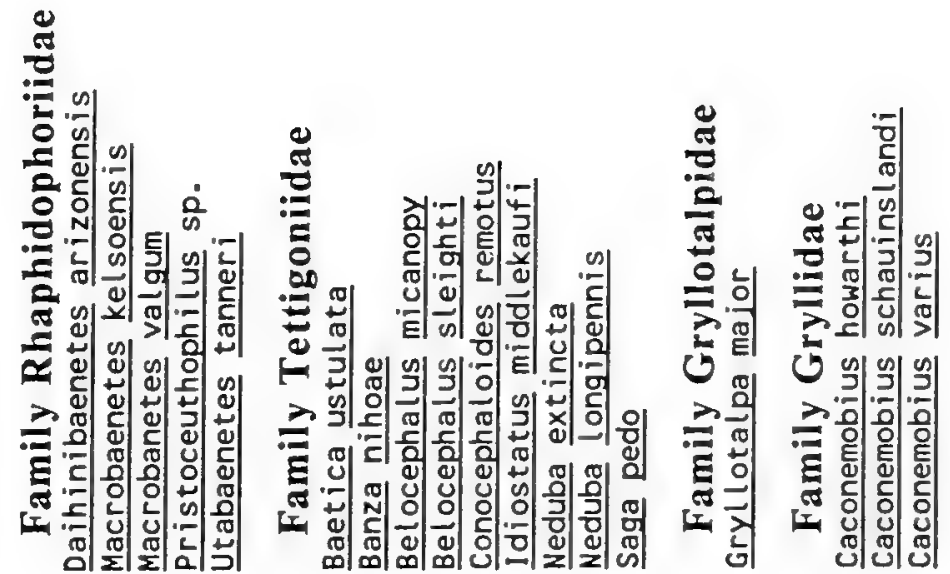


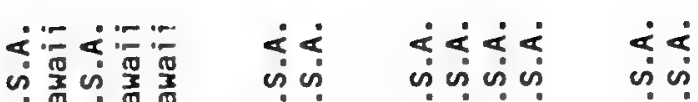

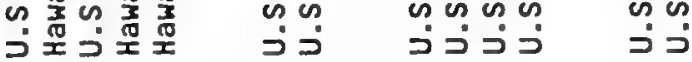
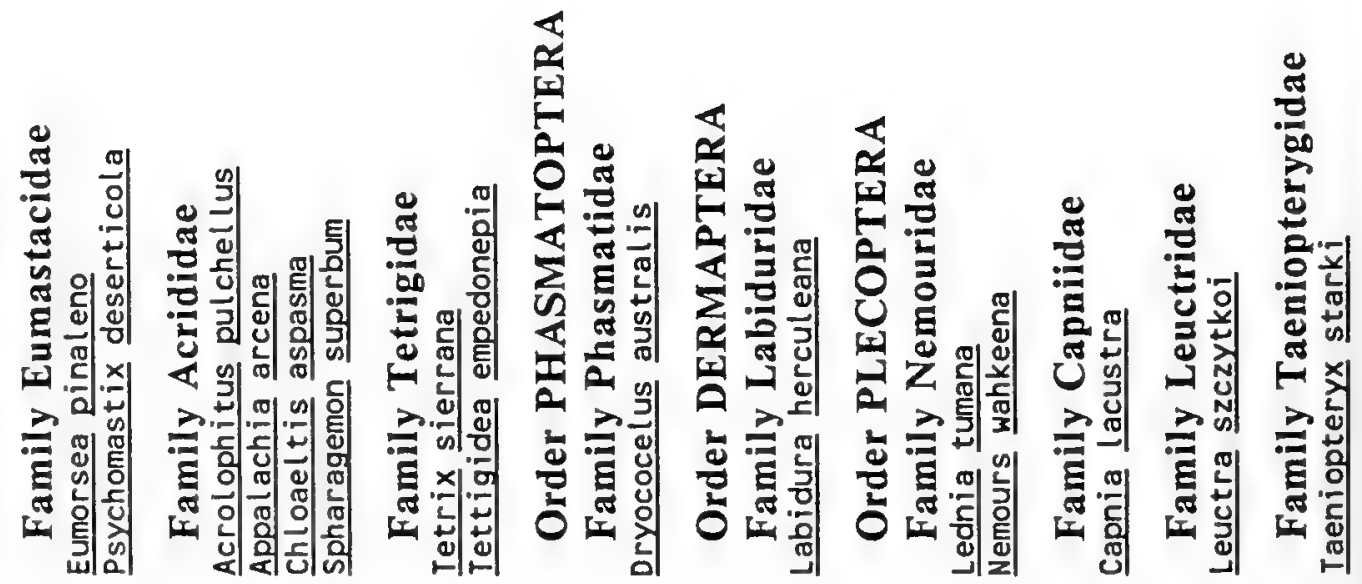


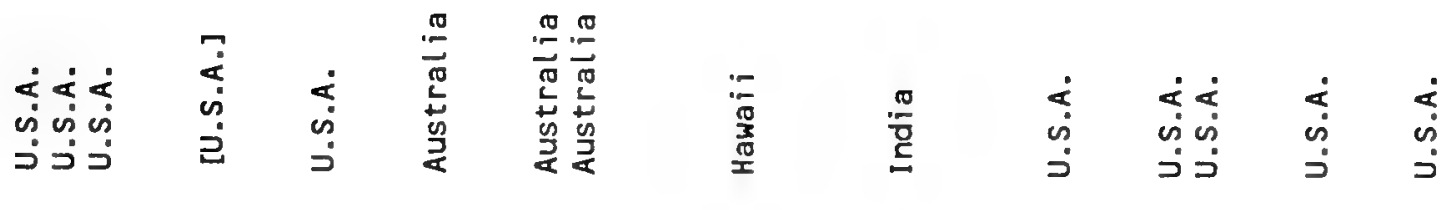

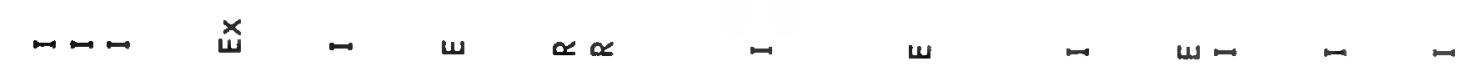
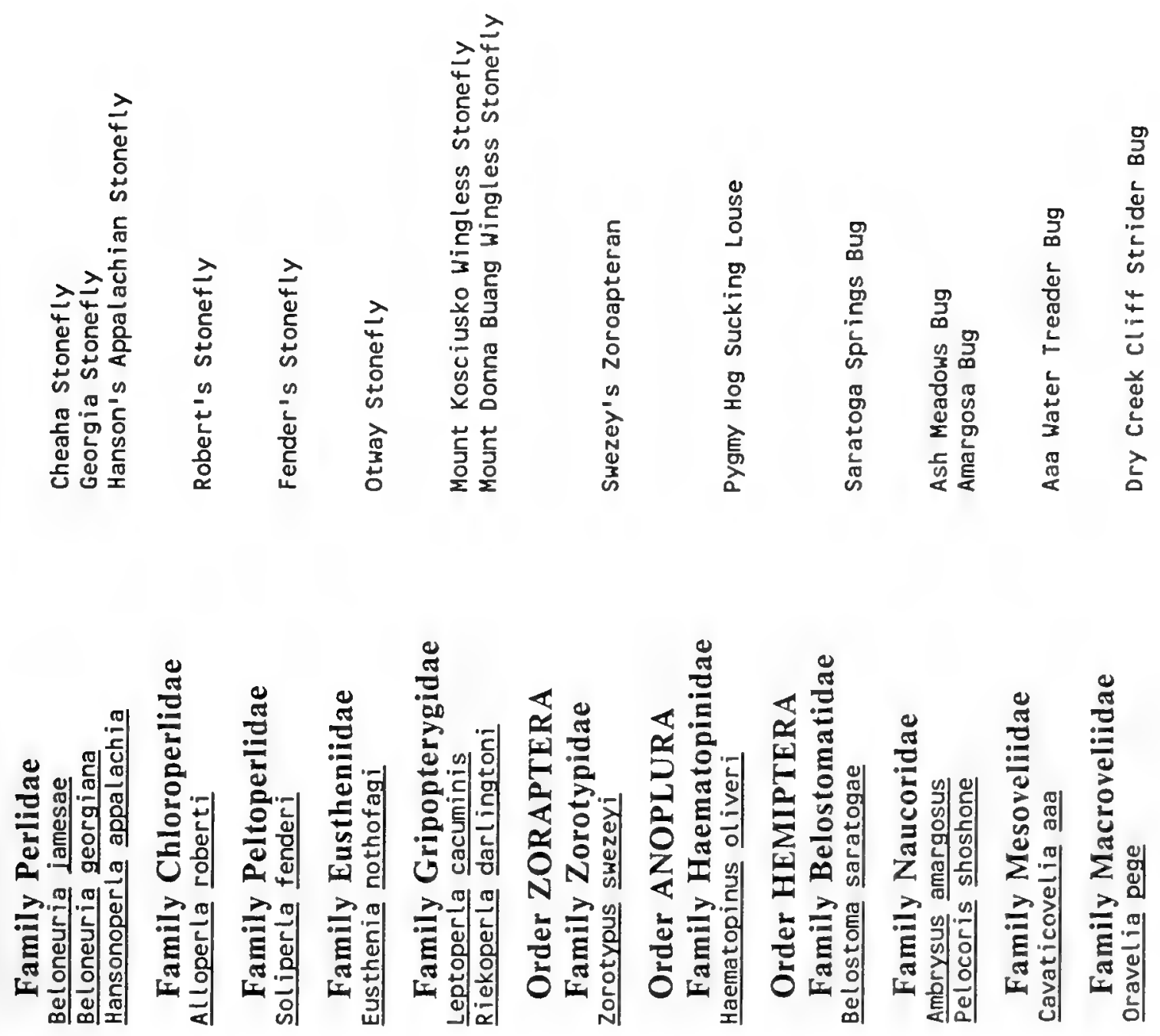


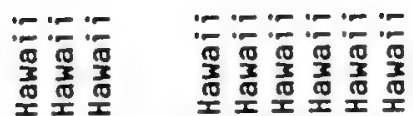

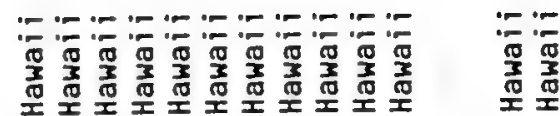

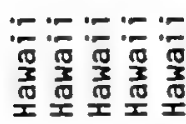

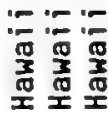

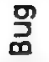

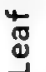

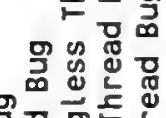

赵

.

蛋号

잉

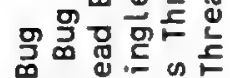

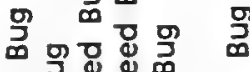

ర్d

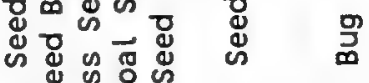

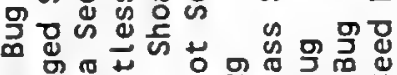

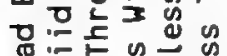

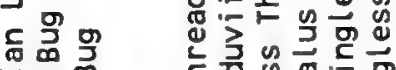

更

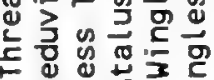

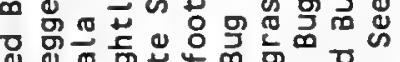

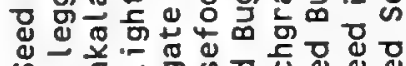

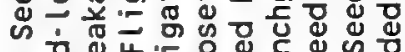

10

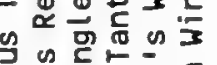

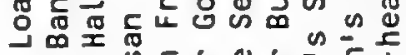

号哭

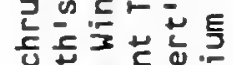

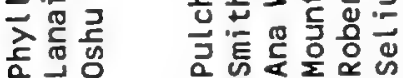

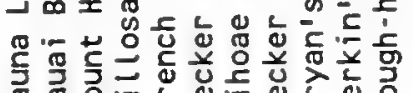

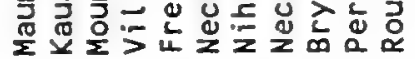

ํํำ 옹

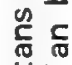

岕:

空

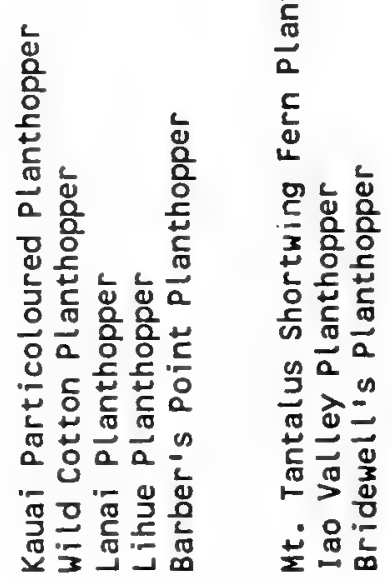

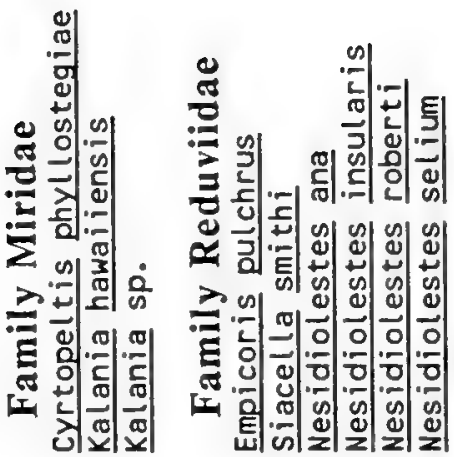
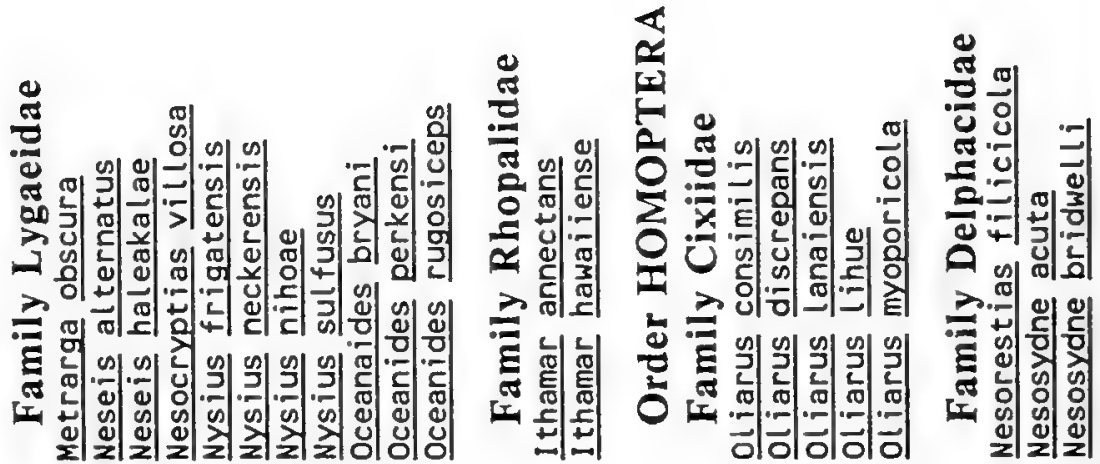


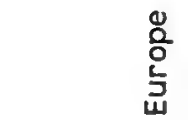

$$
\begin{aligned}
& \dot{i} \quad \frac{2}{2}
\end{aligned}
$$

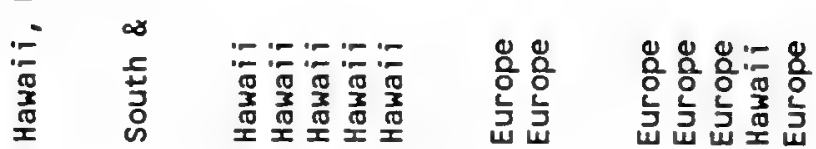

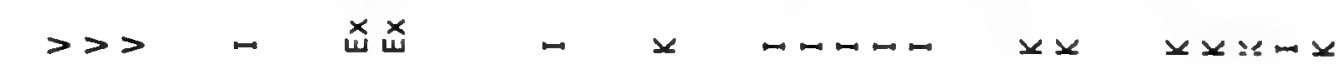

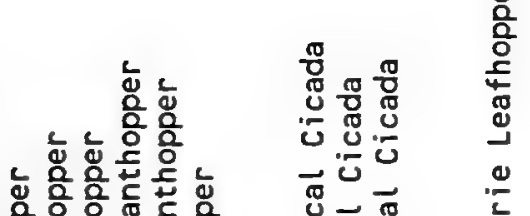

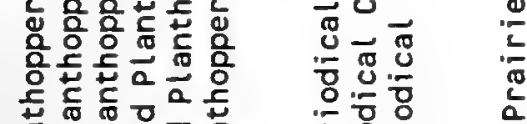

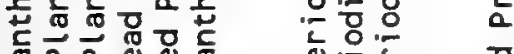

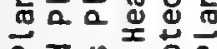
a.

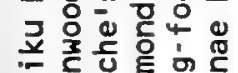

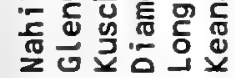

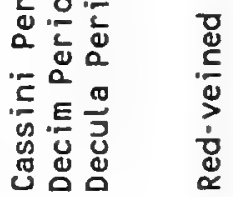

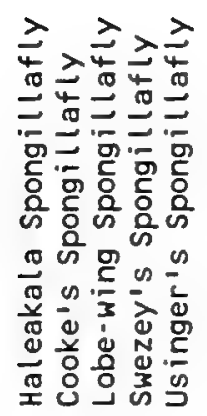

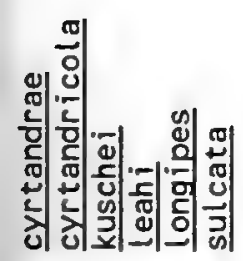

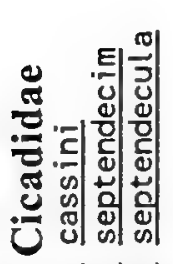

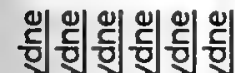

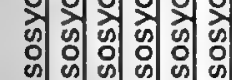

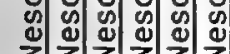

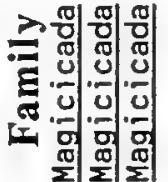

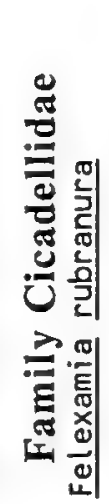

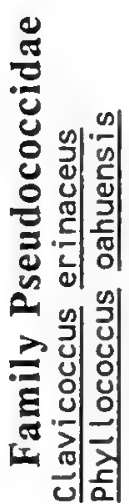

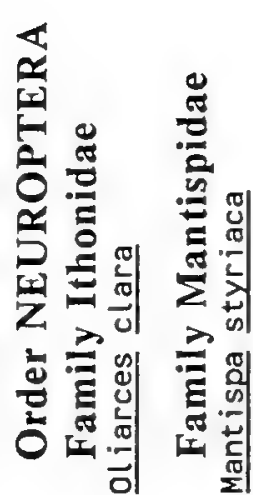
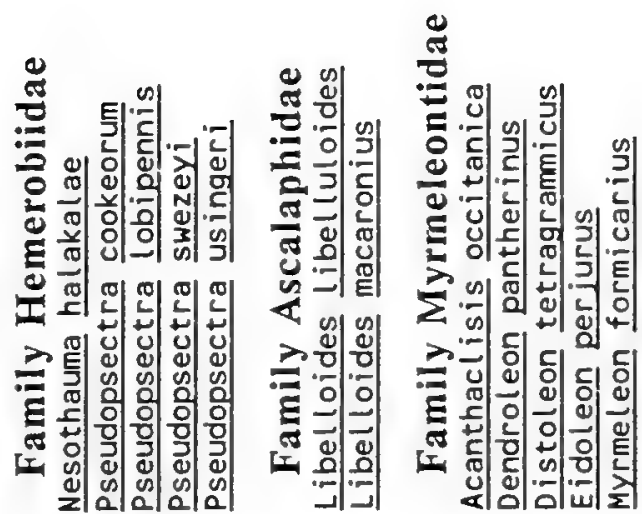

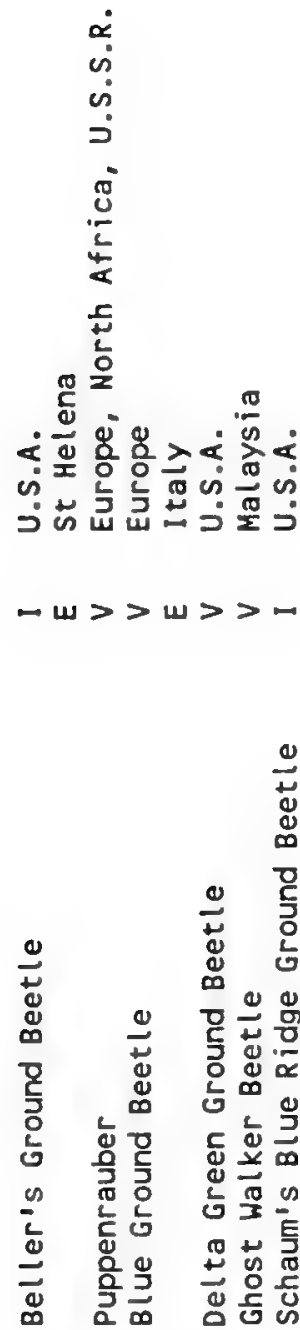

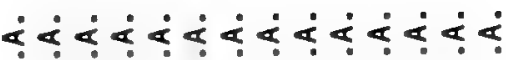

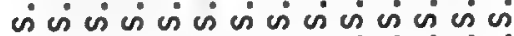

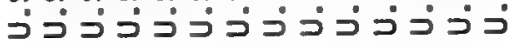

$\frac{\pi}{2}$

离。

$\overrightarrow{2}$

选嵒

ه $\dot{\square} \dot{x}$
is

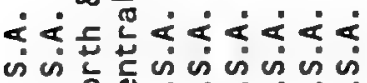

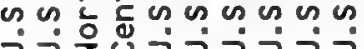
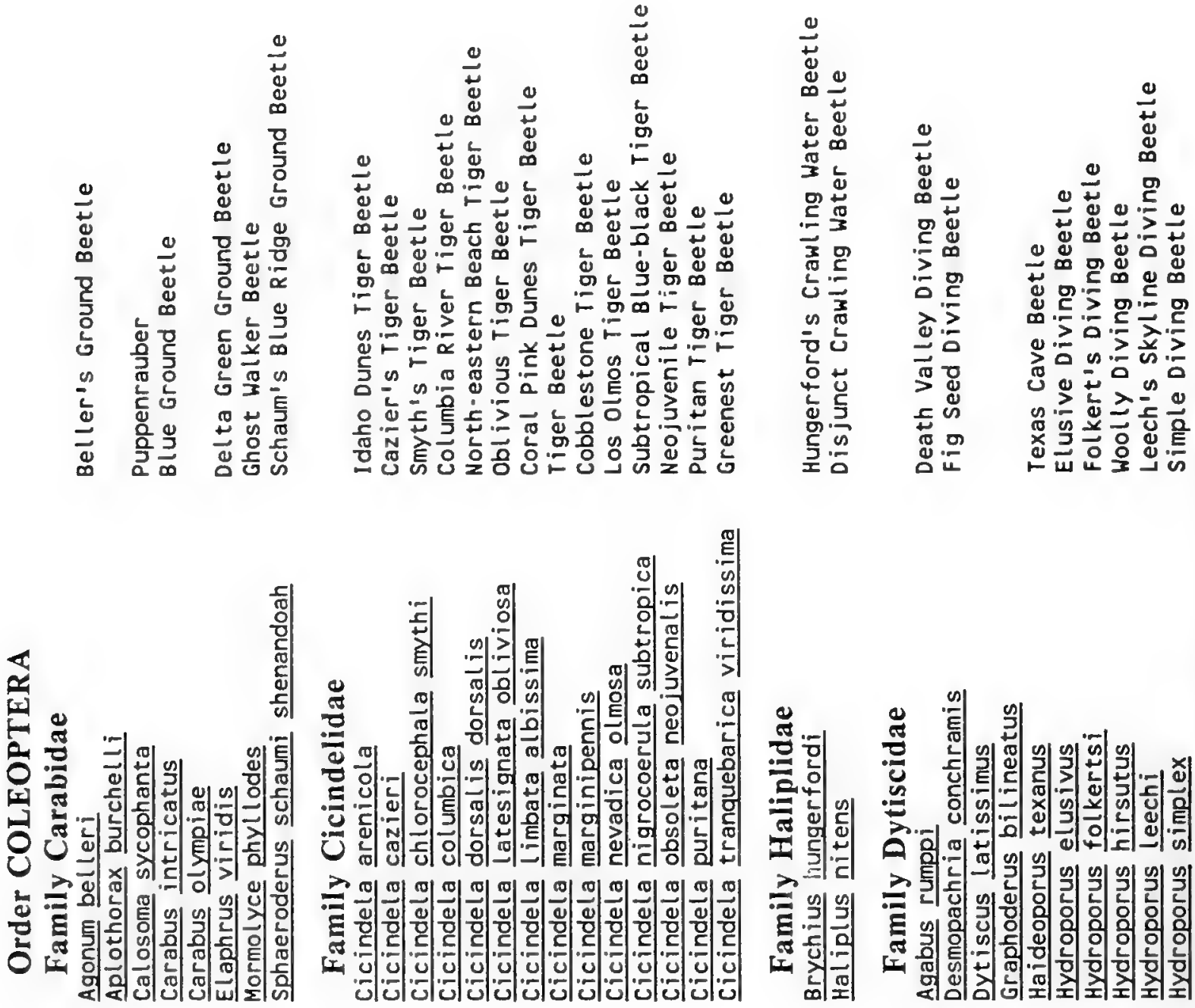

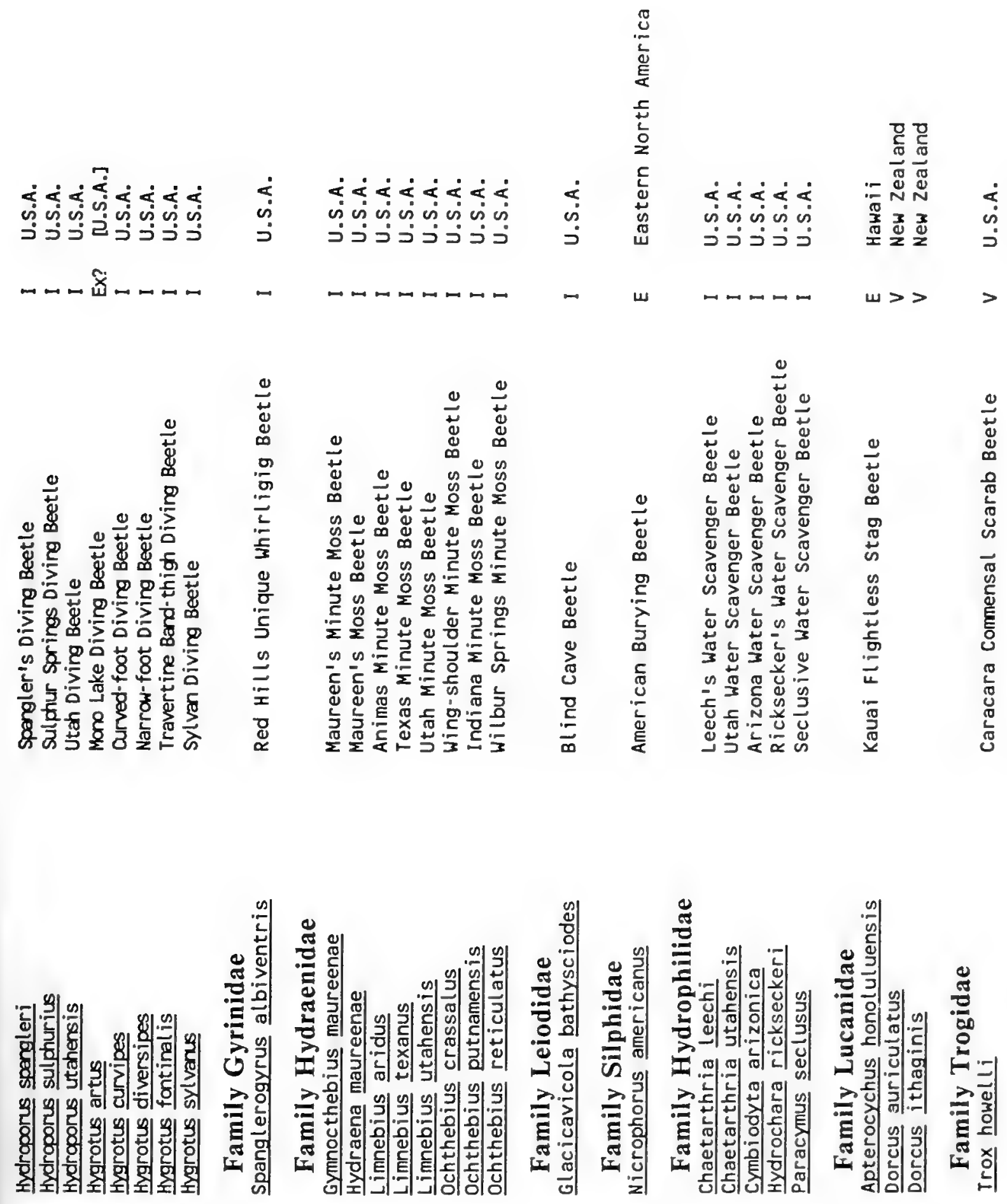


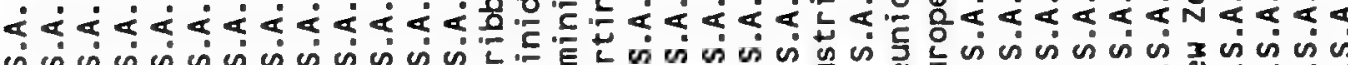
ضُ

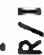

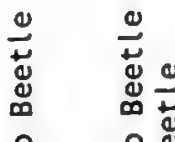

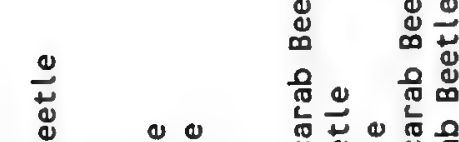
密。

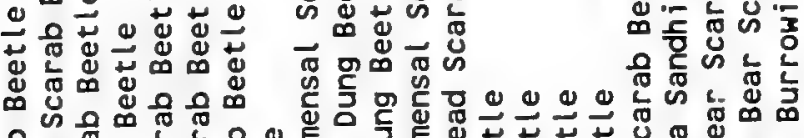

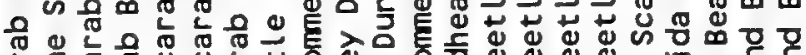

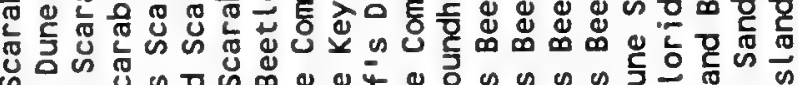

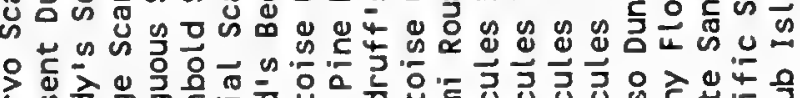

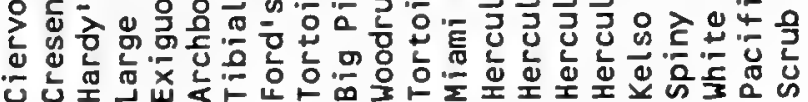

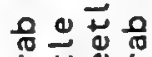

范范京 U

苟二 융

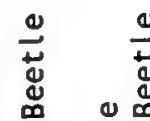

is

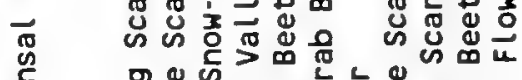

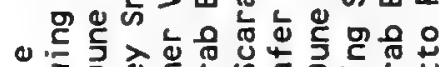

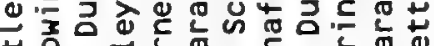

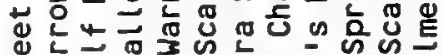

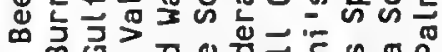

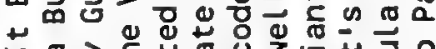

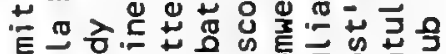

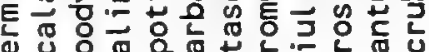

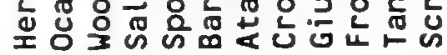

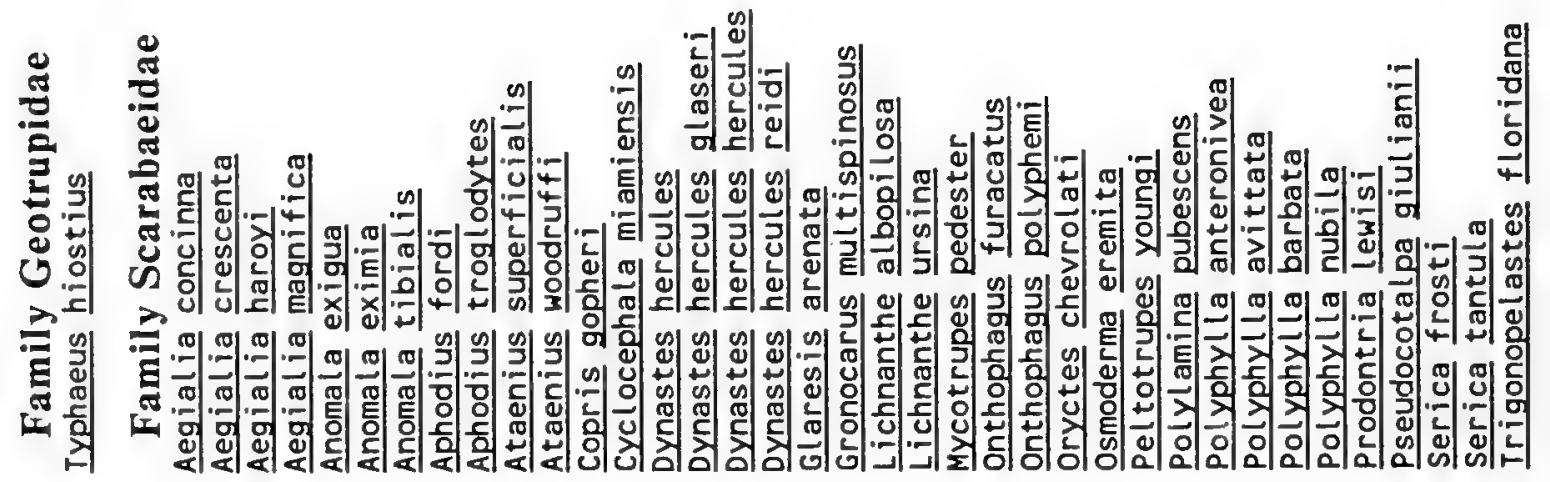




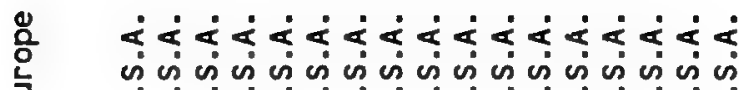

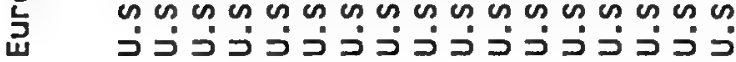

u.

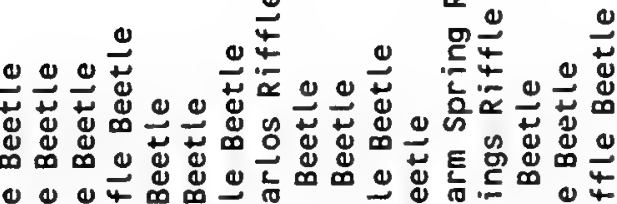

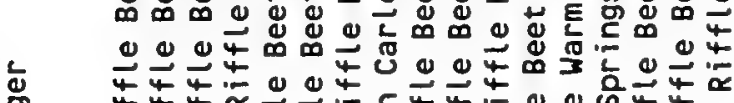

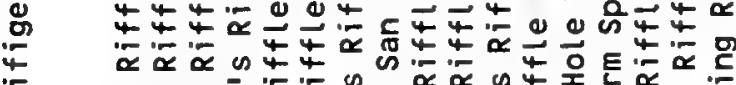

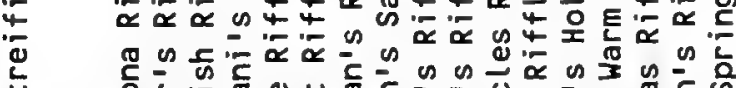

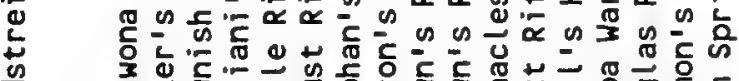

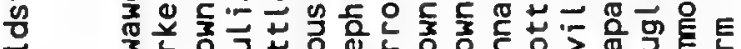
ธำ $\dot{<} \dot{<} \dot{\alpha} \dot{\alpha} \dot{\alpha}$ is nisus is ندذ

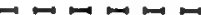

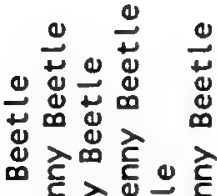

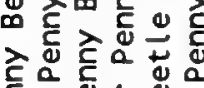
ट⿺辶寸 $0 \stackrel{4}{+\infty}$

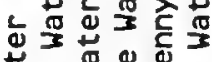

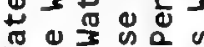

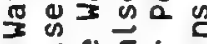

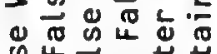

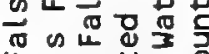
$4=\omega$ 幽

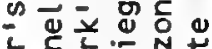

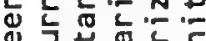
品出出市兵

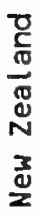

is $\frac{\mathrm{g}}{\mathrm{c}}$

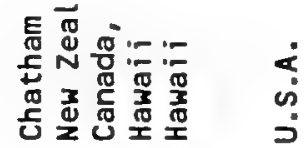

$>>-\omega \curvearrowleft \mapsto$

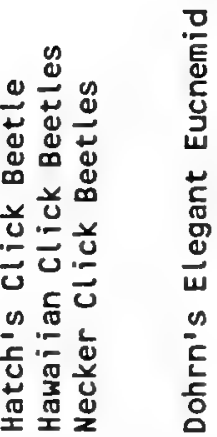
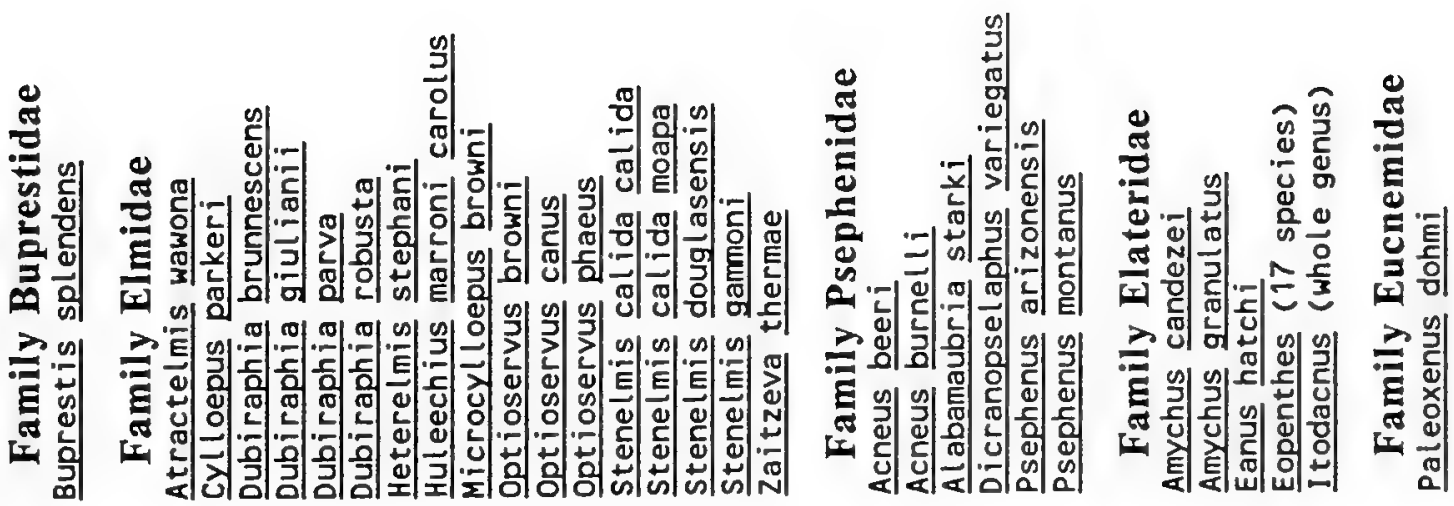


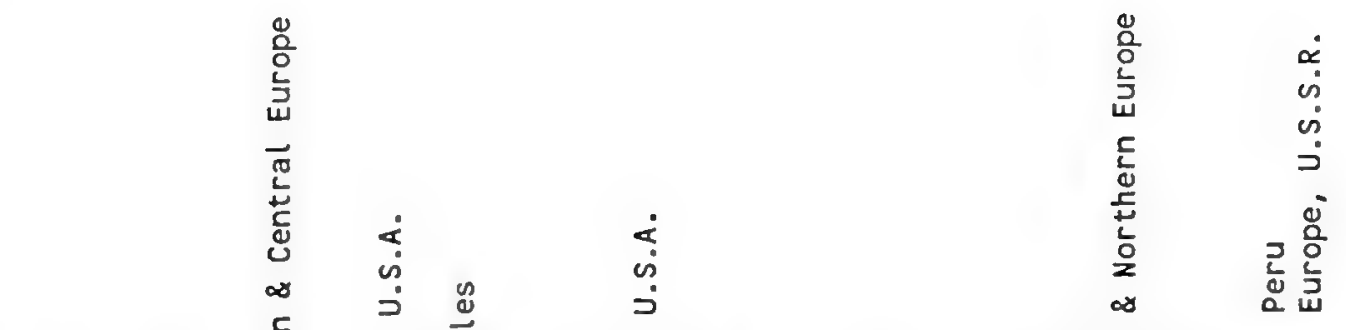

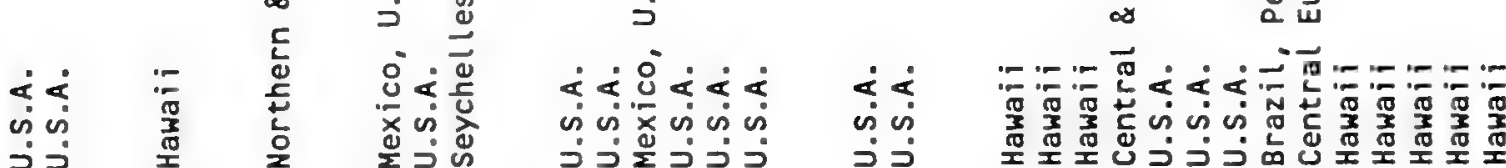

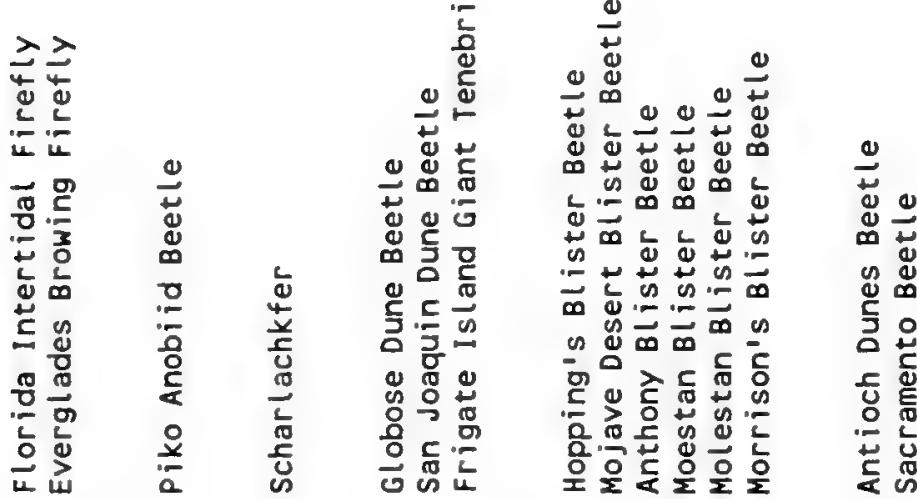
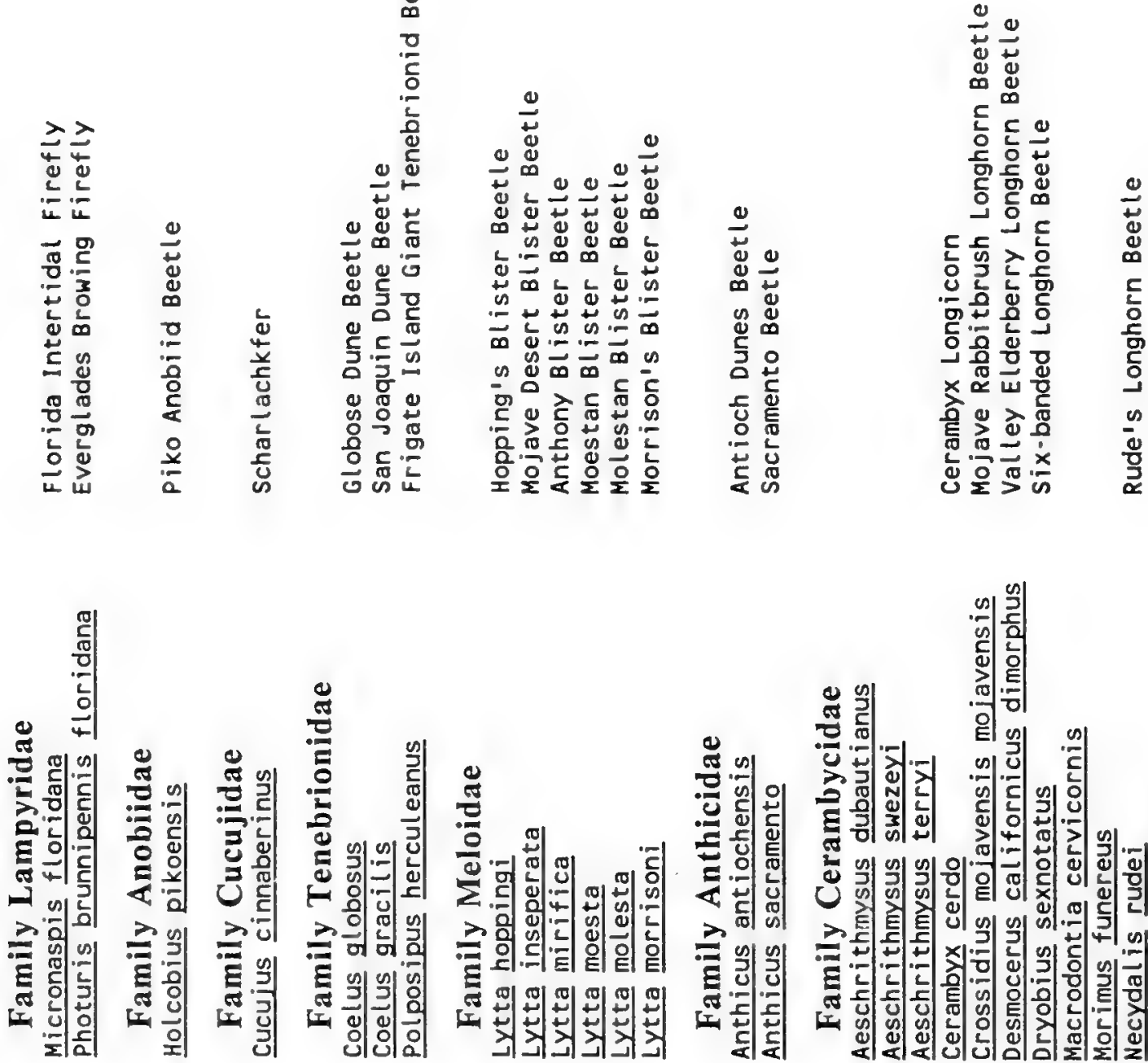

$\frac{0}{0}$
$\frac{2}{2}$
$\frac{2}{2}$
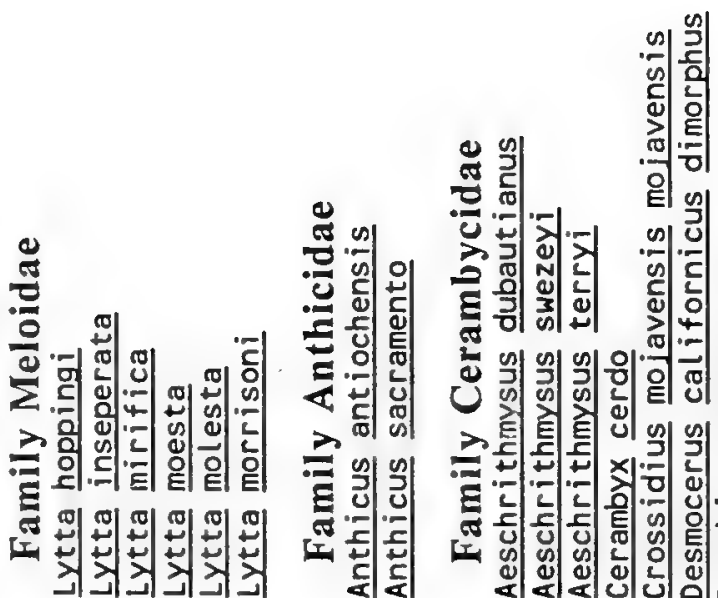

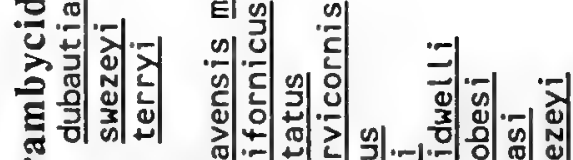

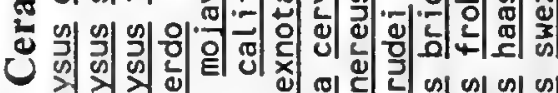
$>$ 리 칠ㅊ

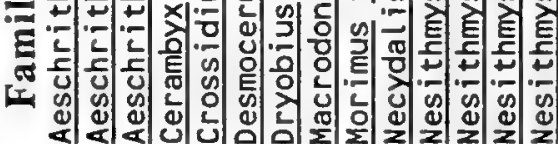




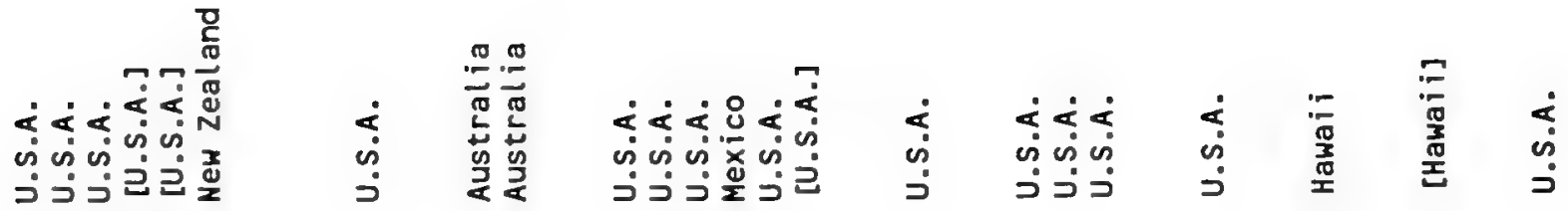

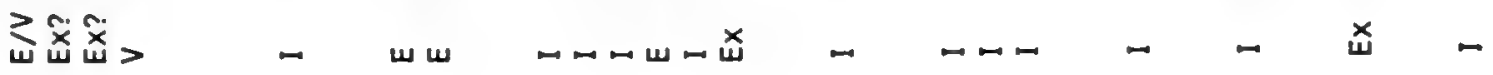
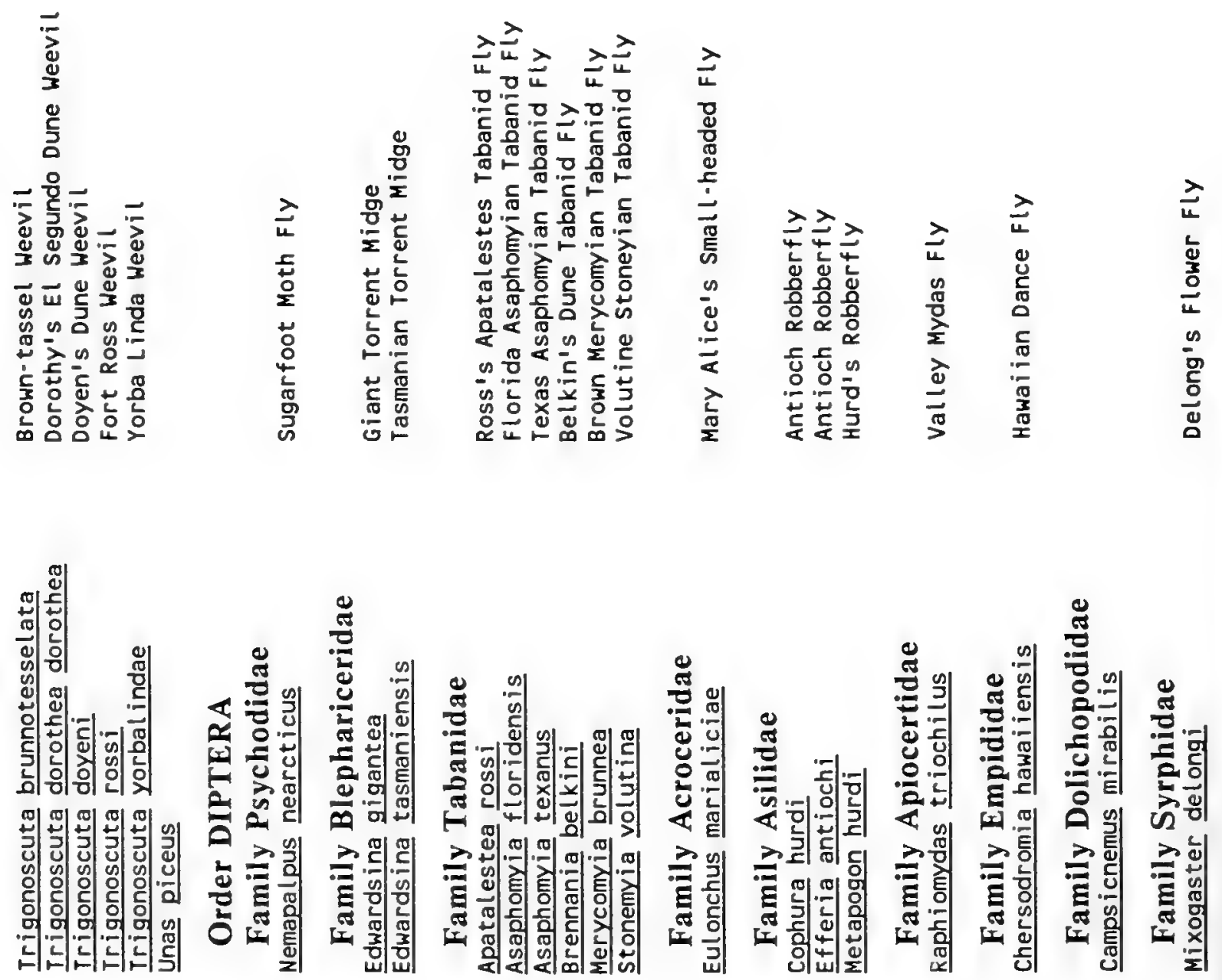


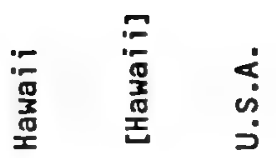

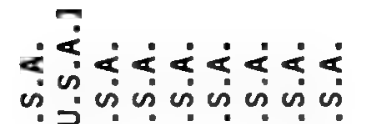

ن

$\dot{4} \dot{<} \dot{<}<\dot{\alpha}$

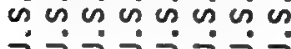

<षं

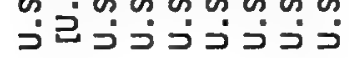

in w on

- 希 -

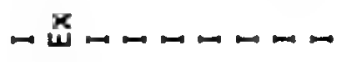

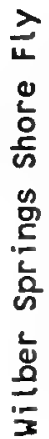

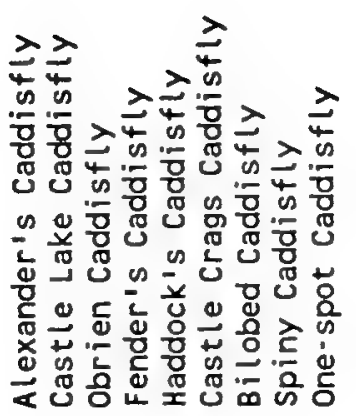

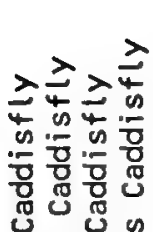
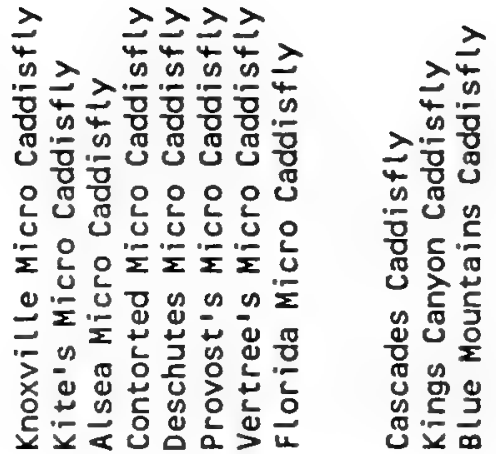

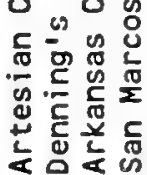

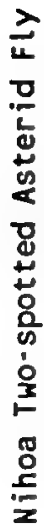
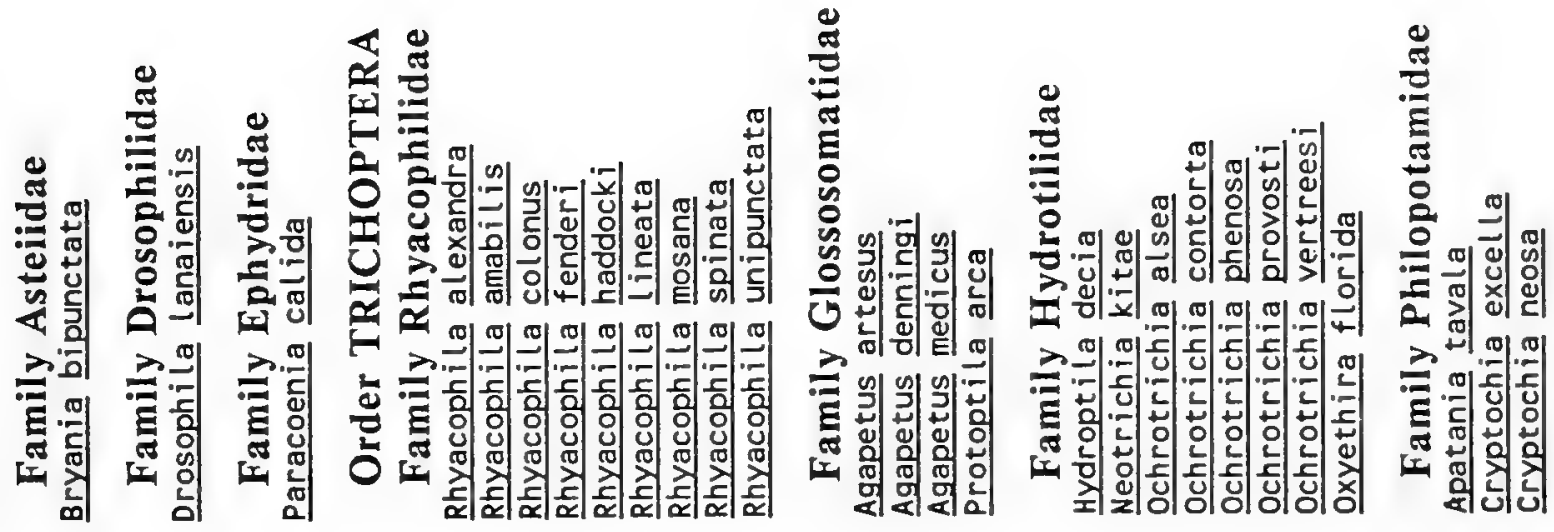


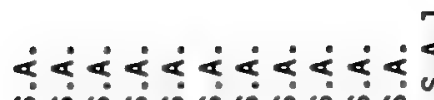

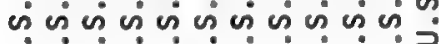

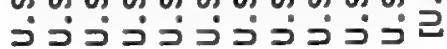

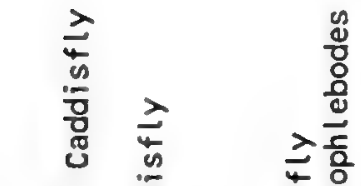

츠도 훙

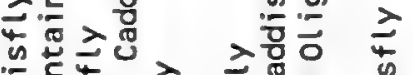
苛

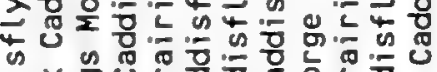

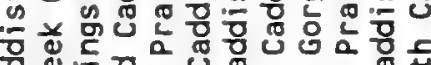

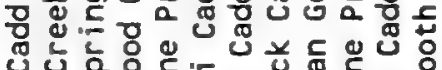

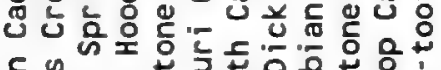

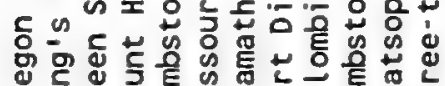

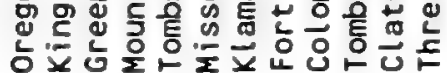

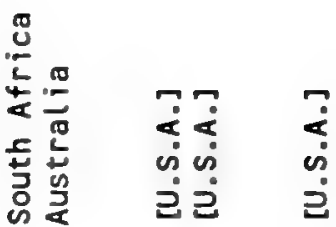

$>>$ 宷妾

\begin{tabular}{|c|c|c|}
\hline 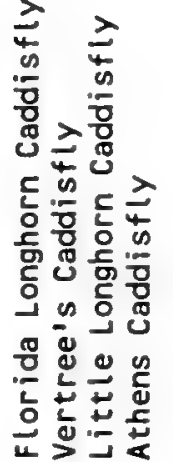 & 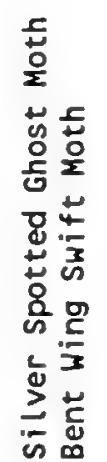 & 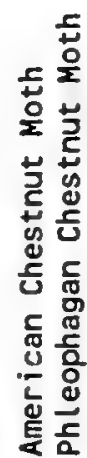 \\
\hline
\end{tabular}
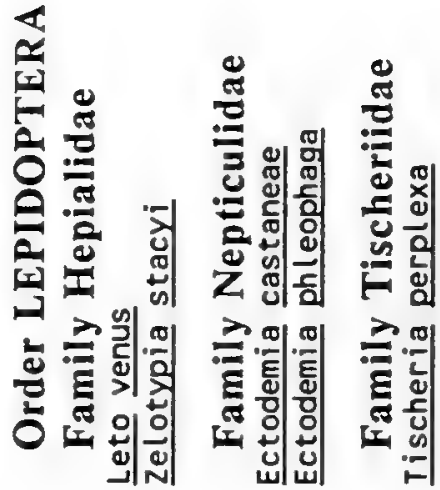


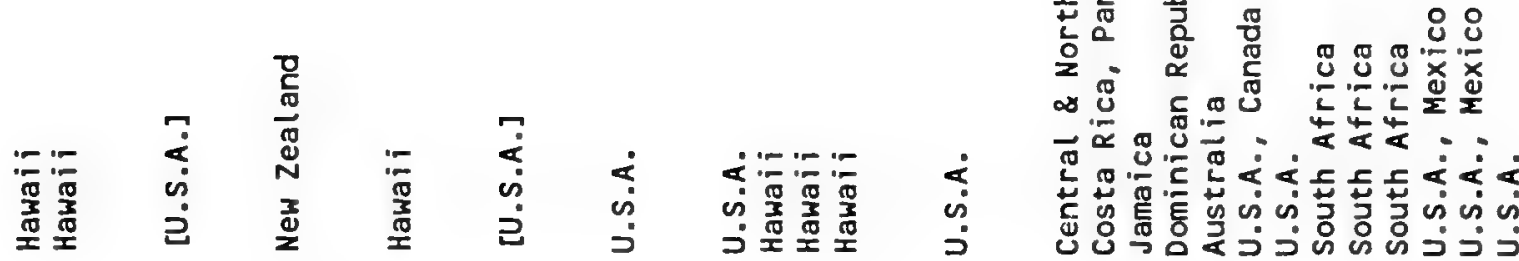

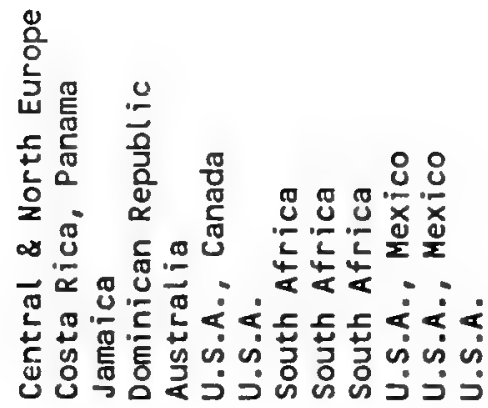

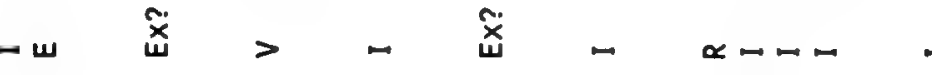

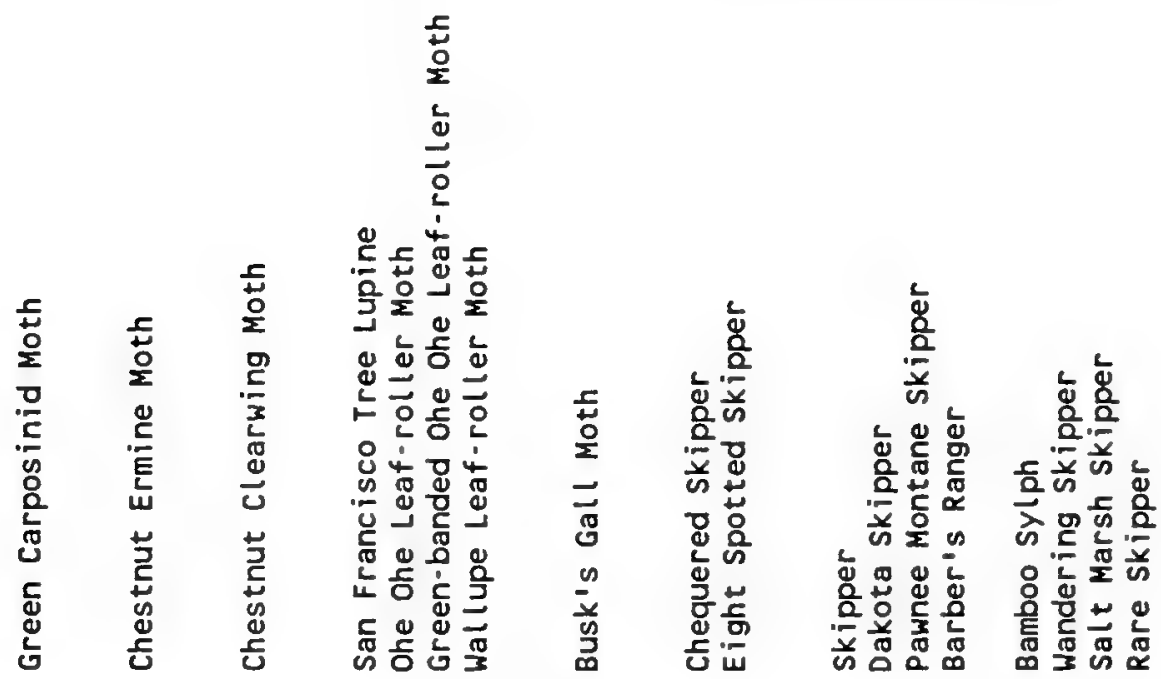

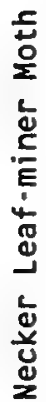
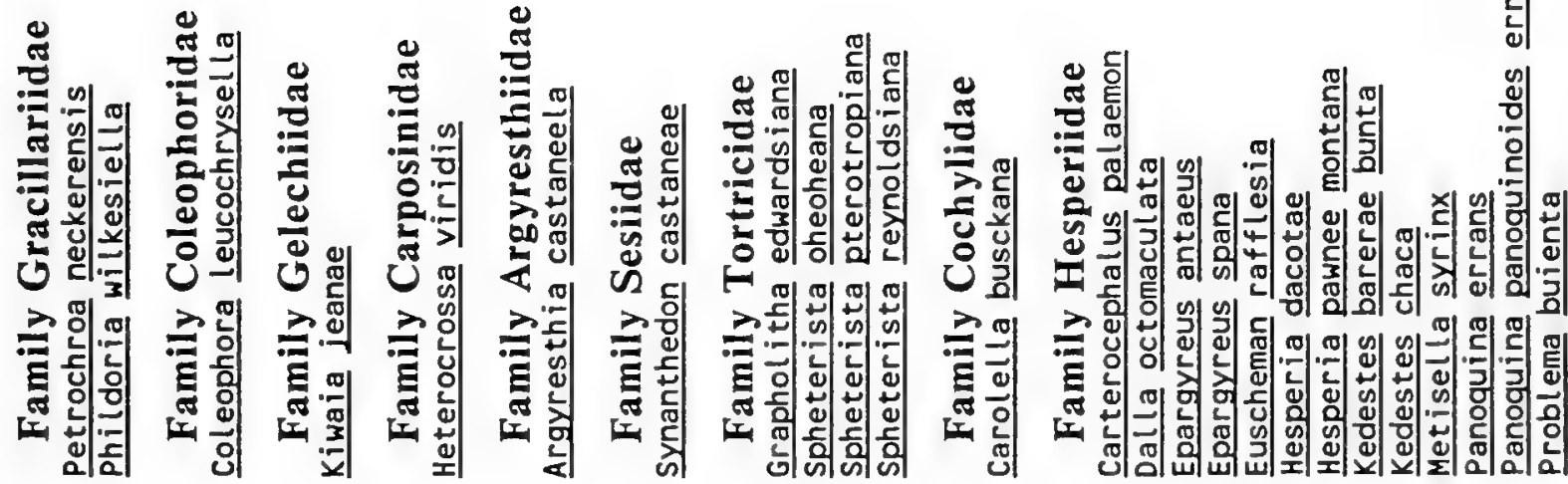


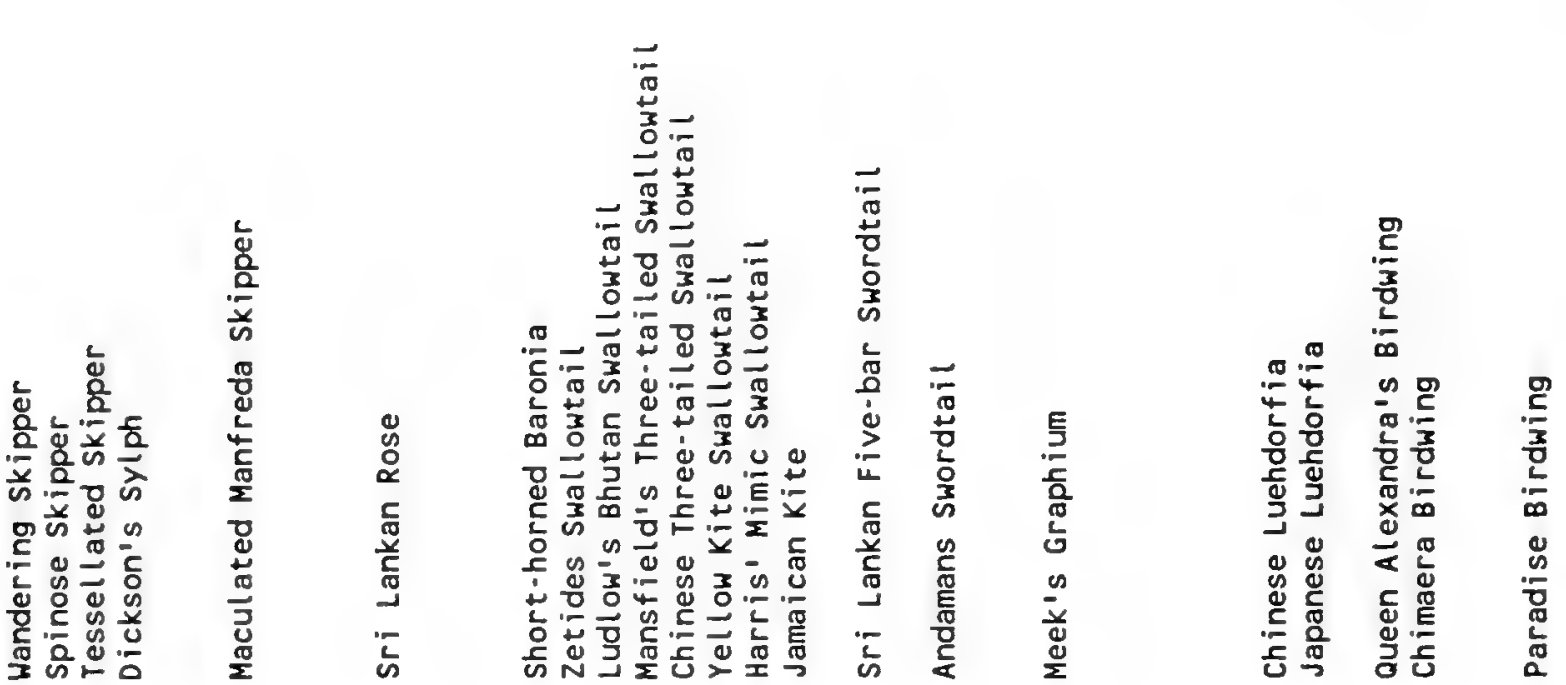
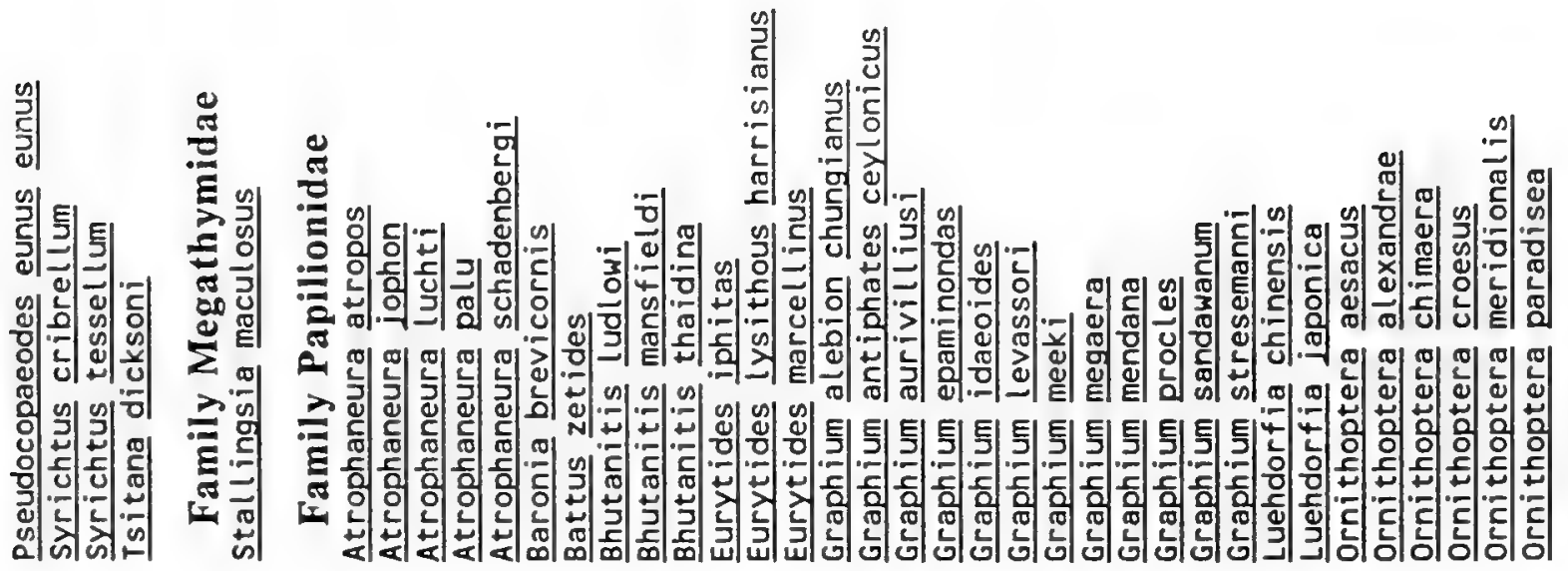
$\frac{\pi}{\frac{0}{4}}$

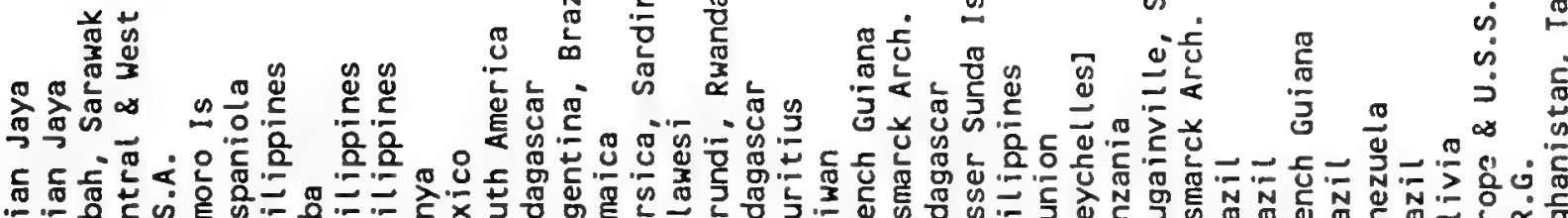

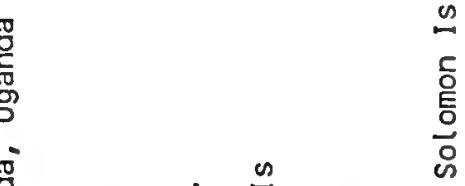

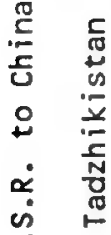

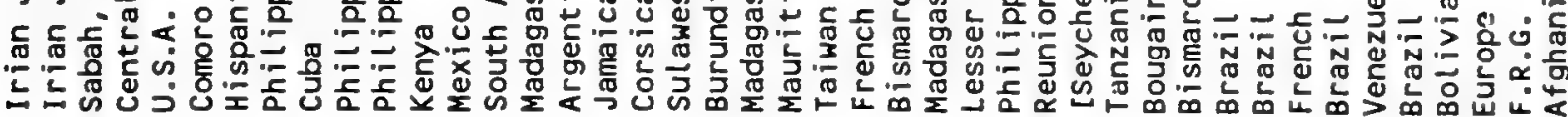

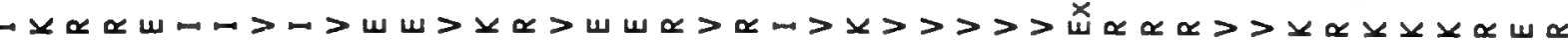

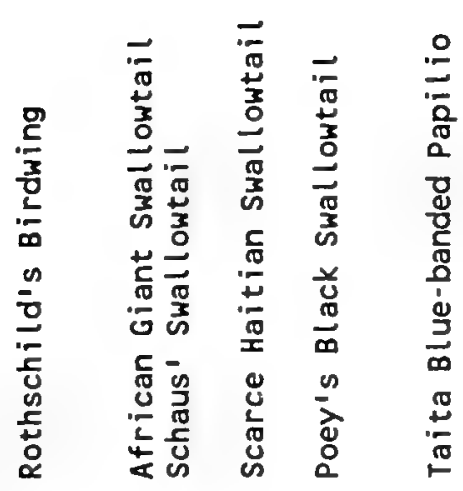

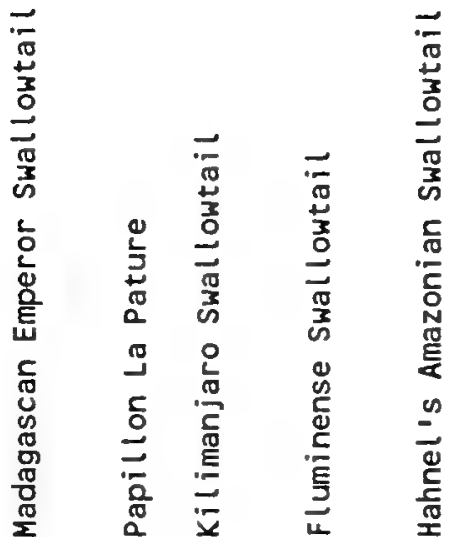

$\frac{0}{\frac{0}{2}}$

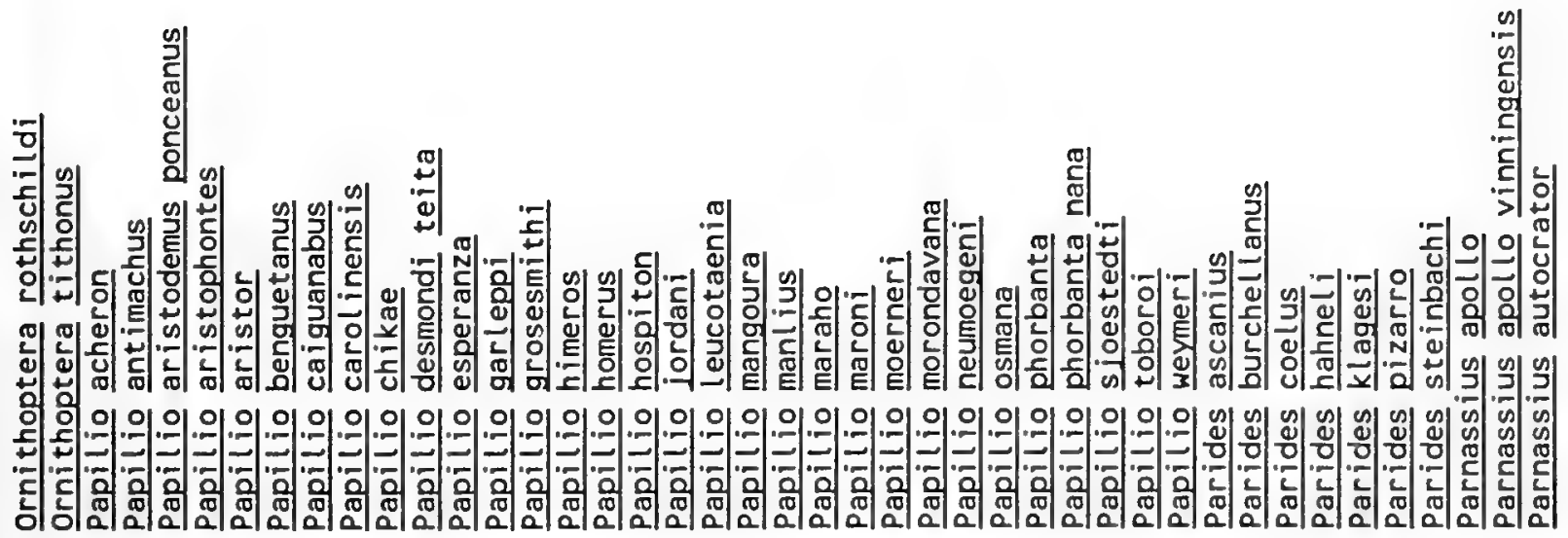




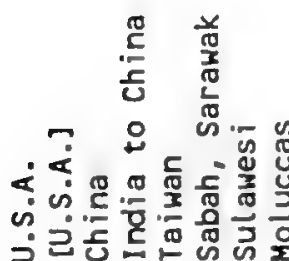

- $\underset{w}{x} \propto w \sim>m$

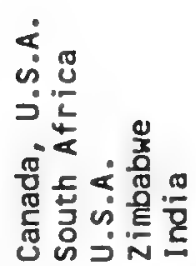

- $-\propto \propto$

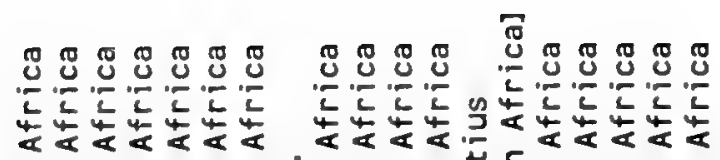

돈

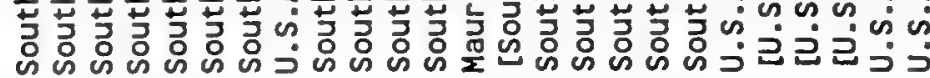

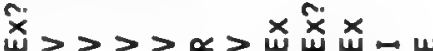
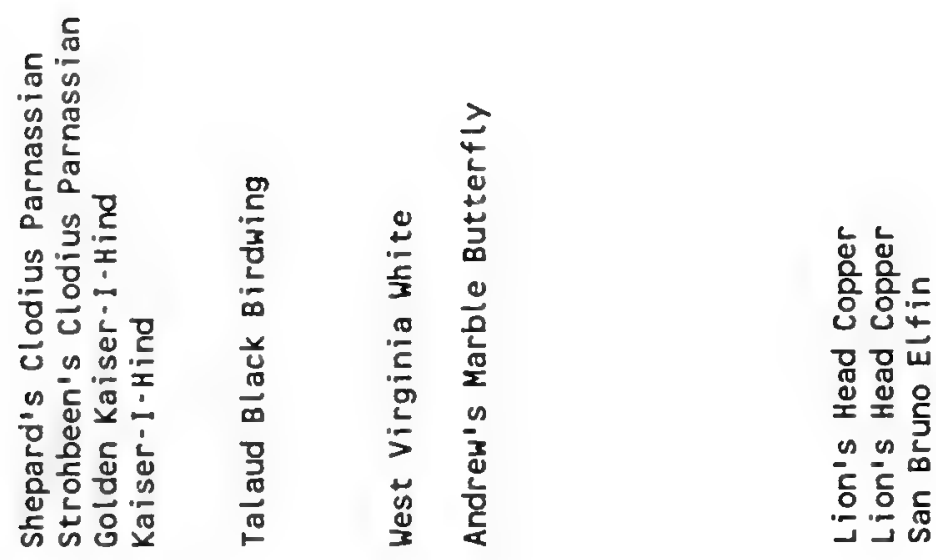

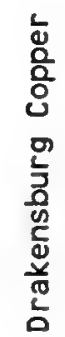
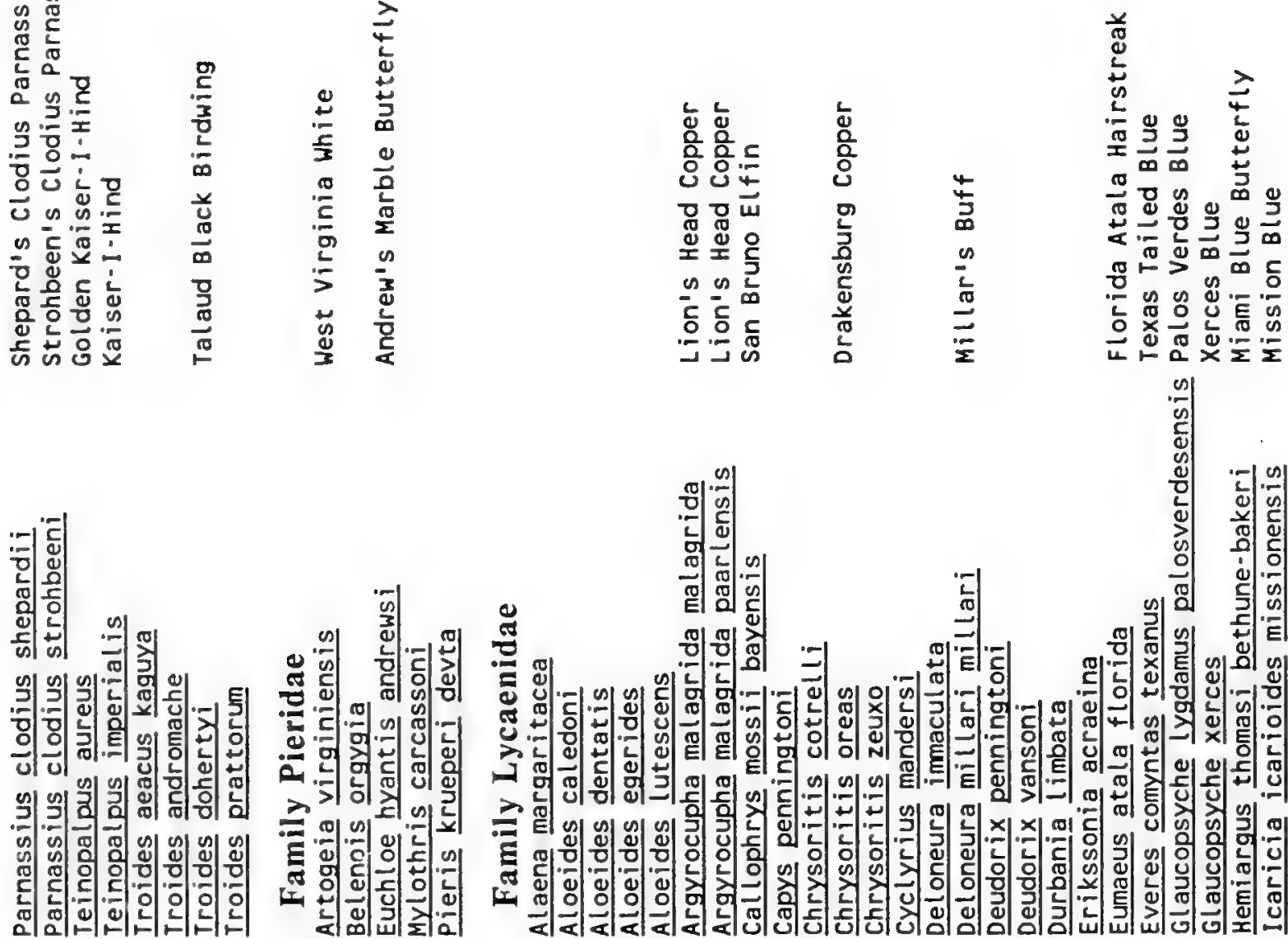

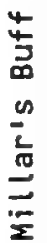

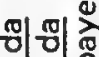

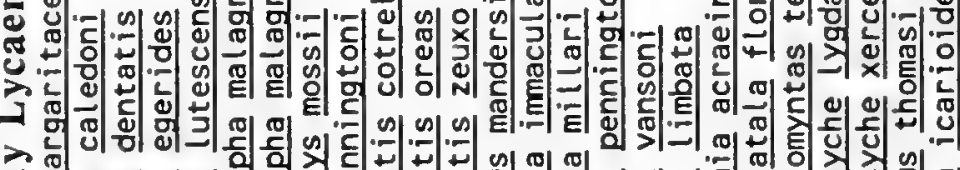
>웡

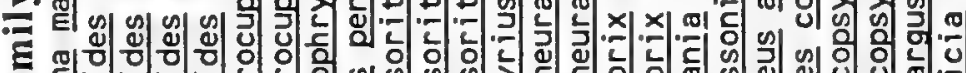

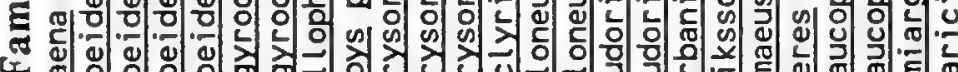

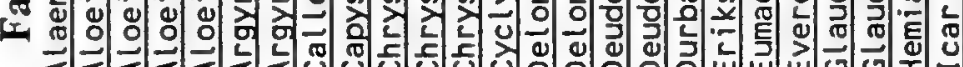




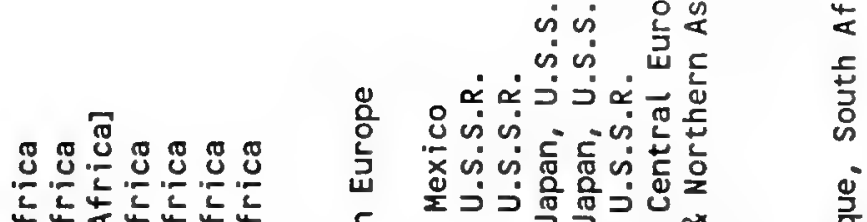

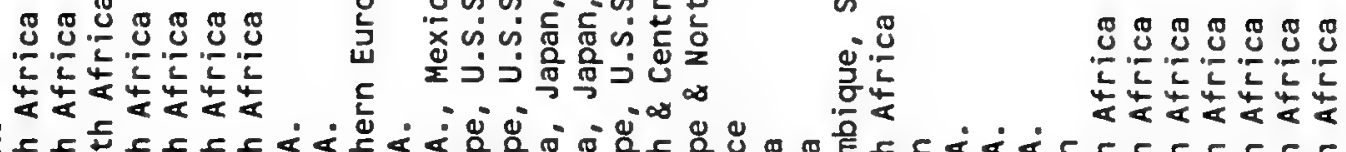

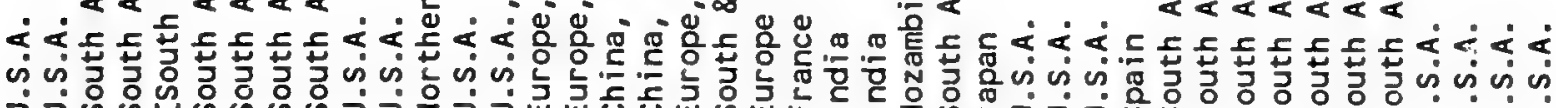
ذد

- - w> ע

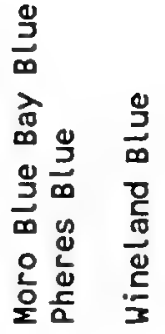

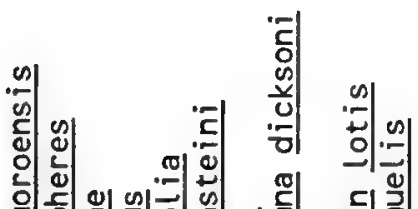

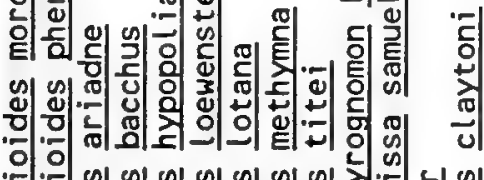

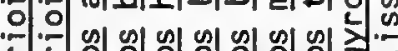

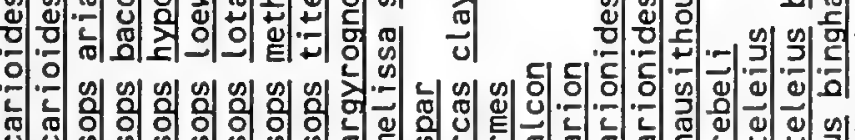

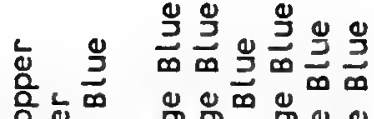

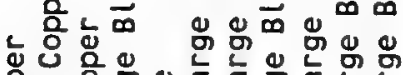

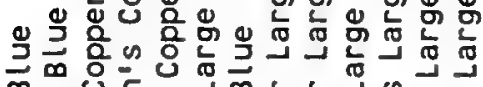

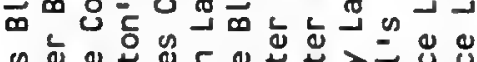

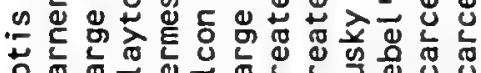

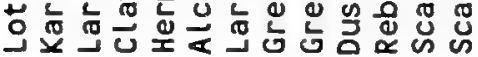

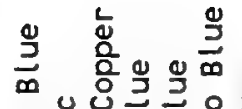

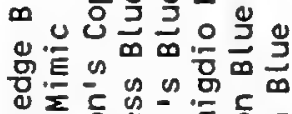

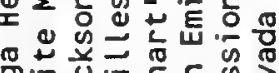

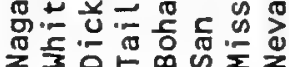

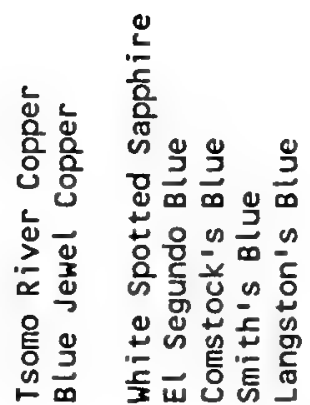

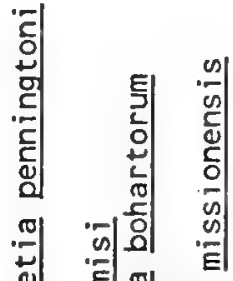

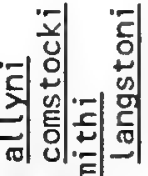
की क्षा को

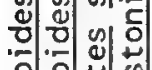

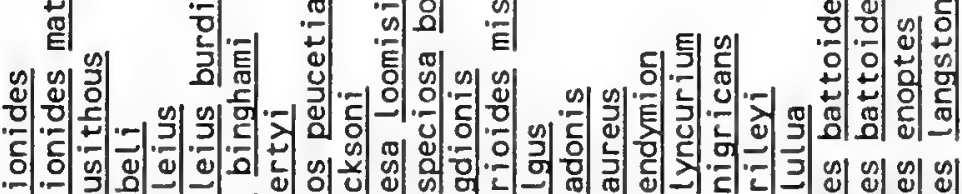

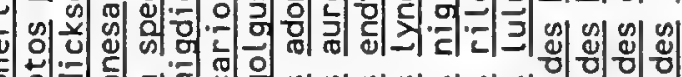

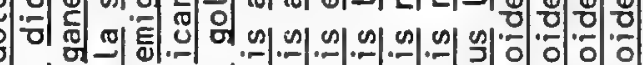

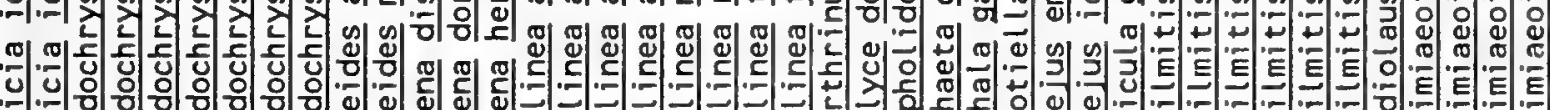
(1)

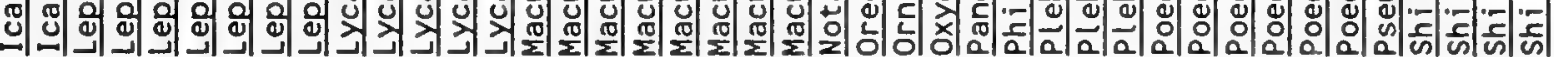




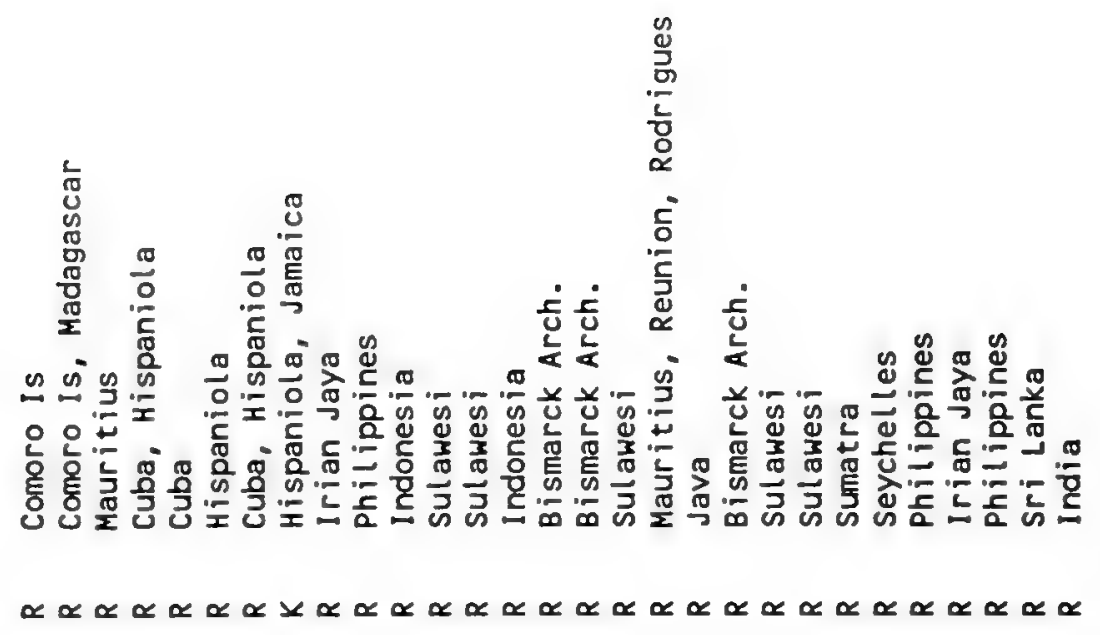

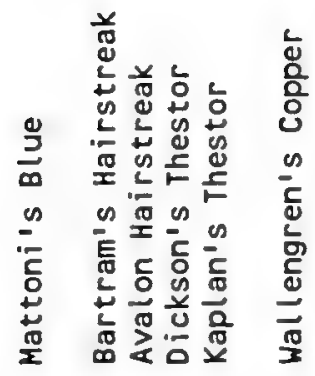

苞

:

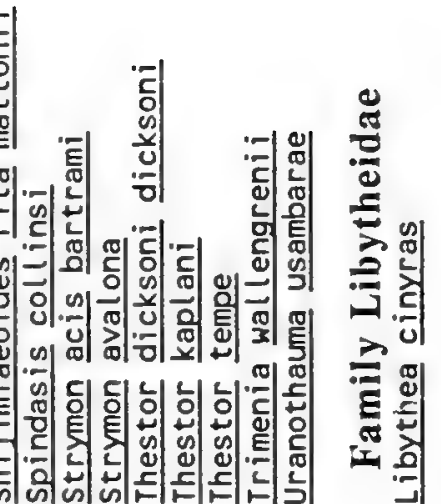

है

刍

อ

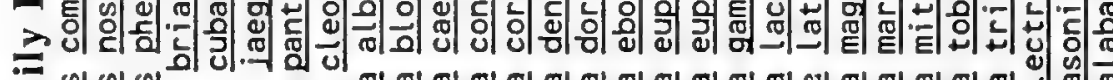

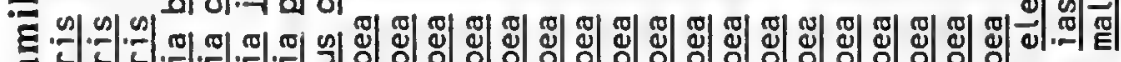

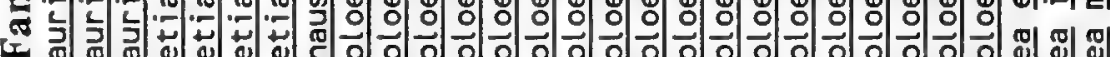

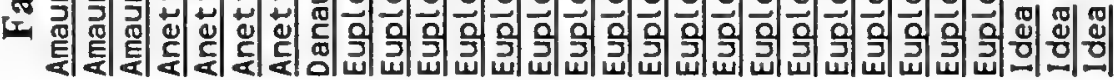




\section{$\stackrel{2}{2}$}

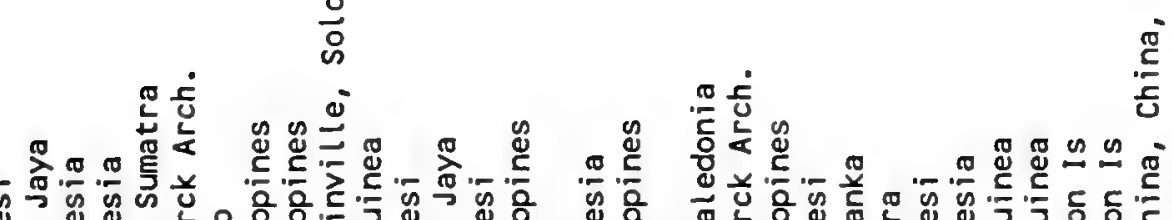
के

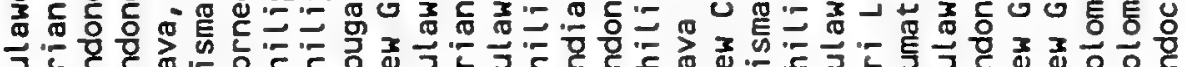

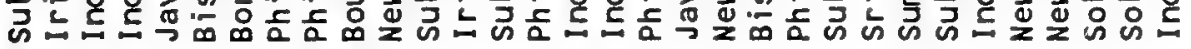

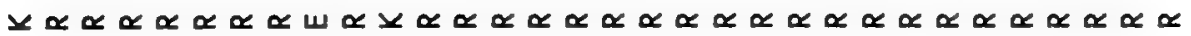

$w \alpha>-\alpha \propto \alpha \propto$

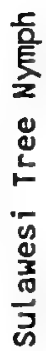

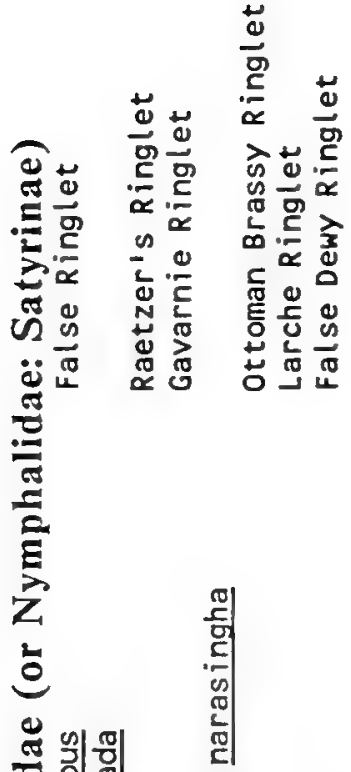

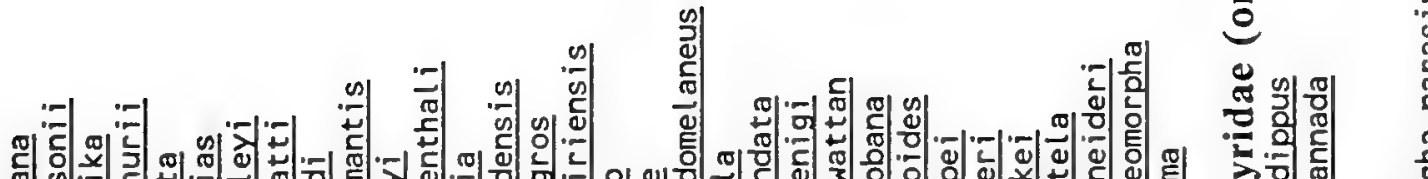
Q

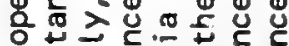

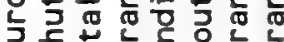

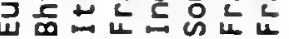

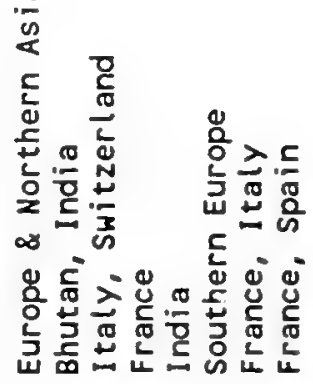

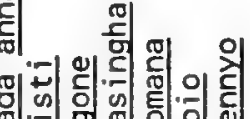

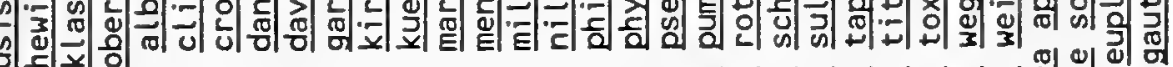




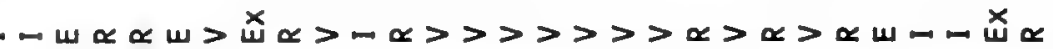

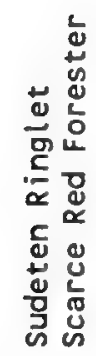
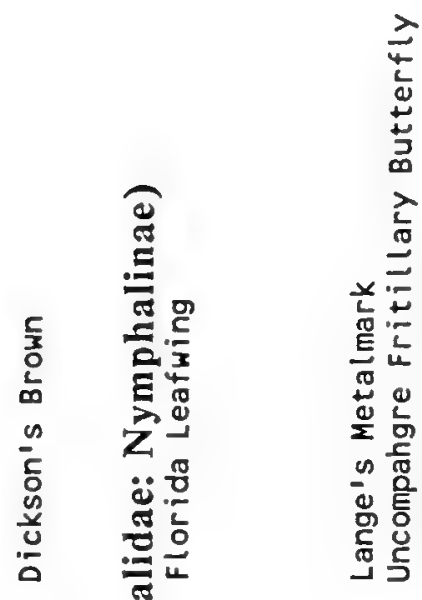

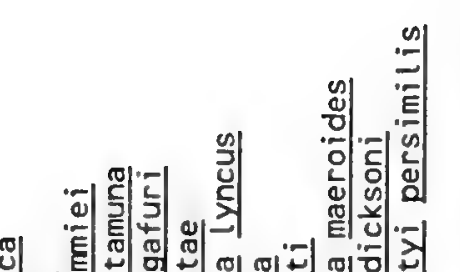

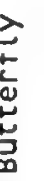

䍃

는

过

닌

돈

嵌荒

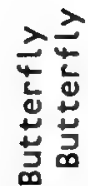

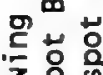

준융

苋这过

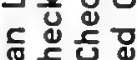

䖪它

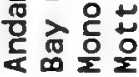

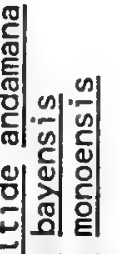

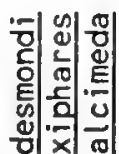

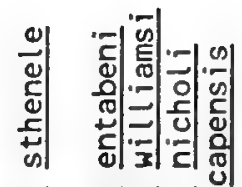

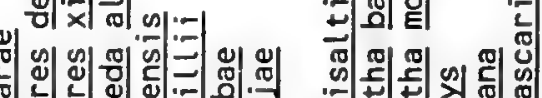

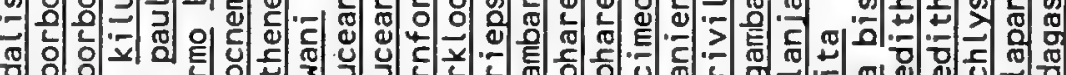

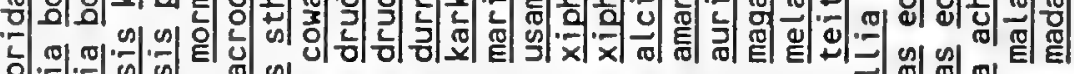

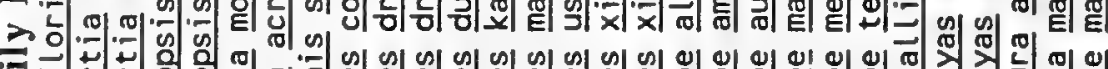

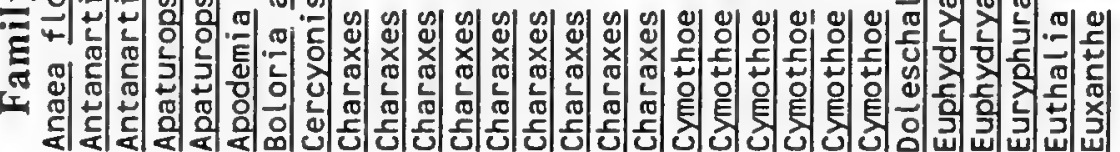



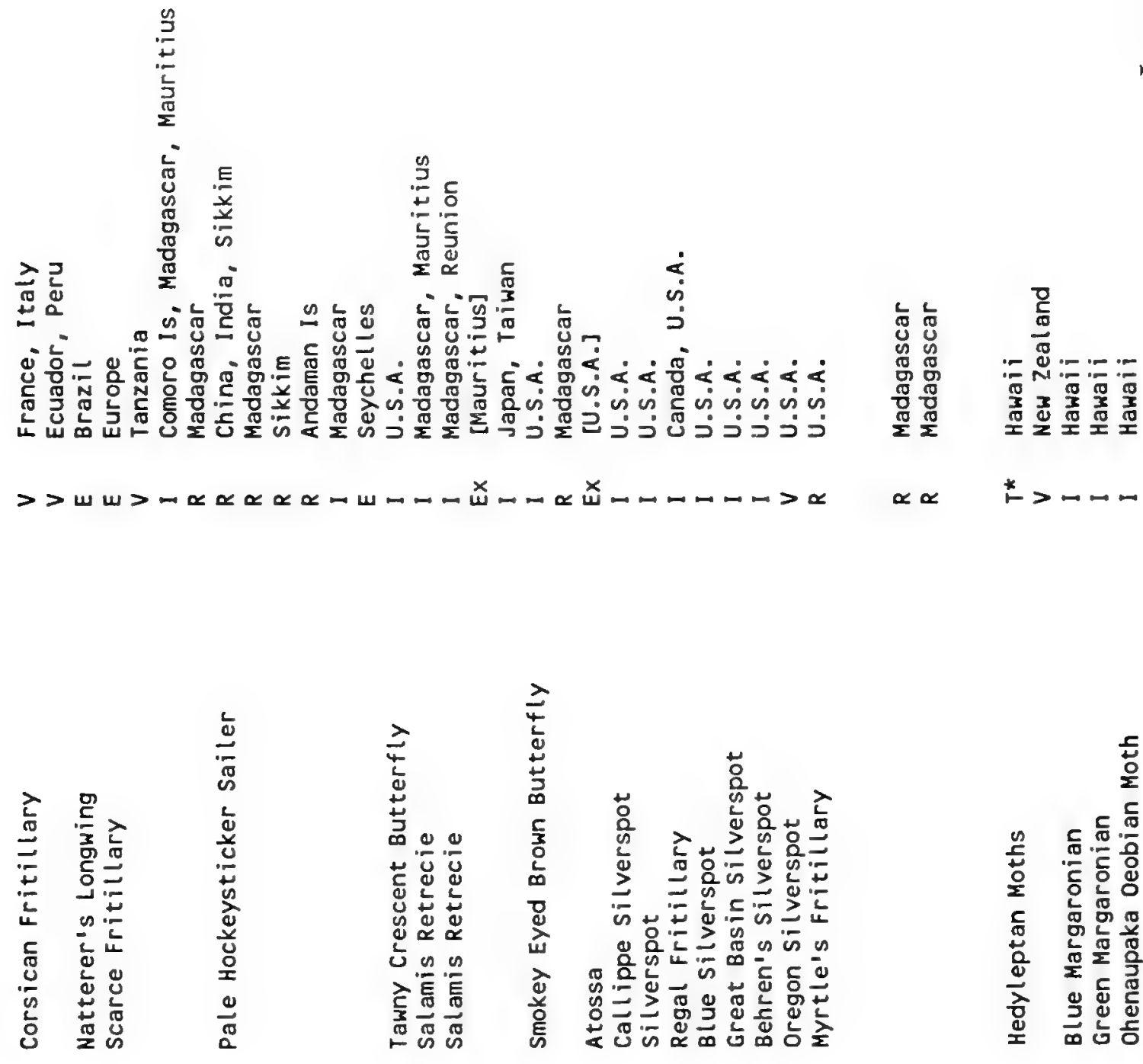

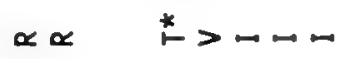
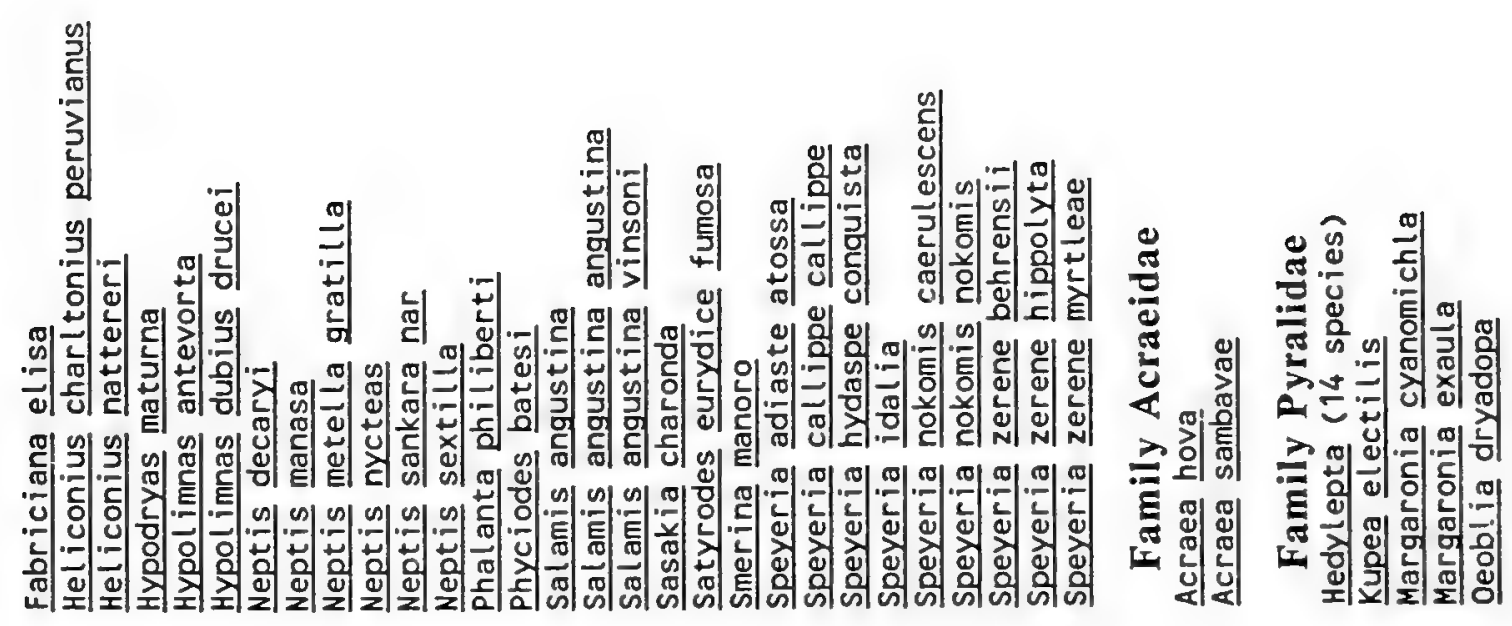


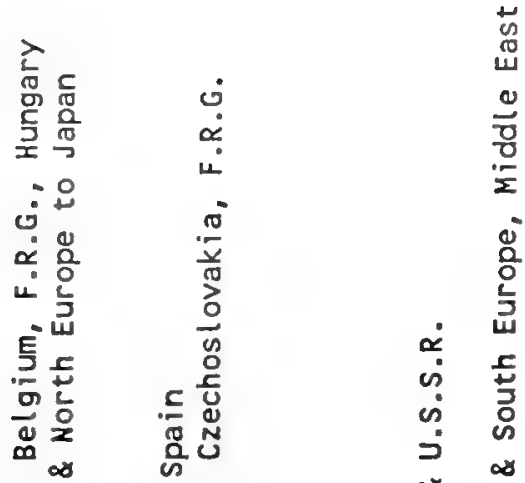

ำ

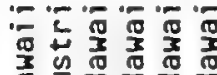

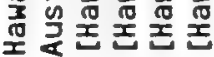

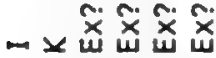

芒苔 खे

$w>\quad>w$ $\infty-\infty$

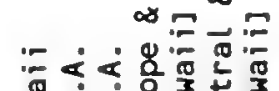

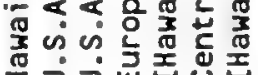
エつつய元U $w>w>\stackrel{\tilde{x}}{w} \underset{\tilde{x}}{\tilde{x}}$

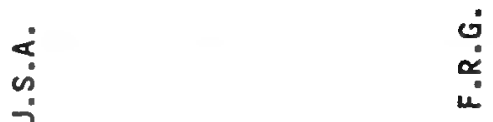

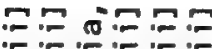
ช。

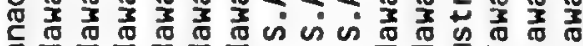

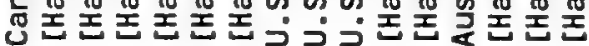
戠
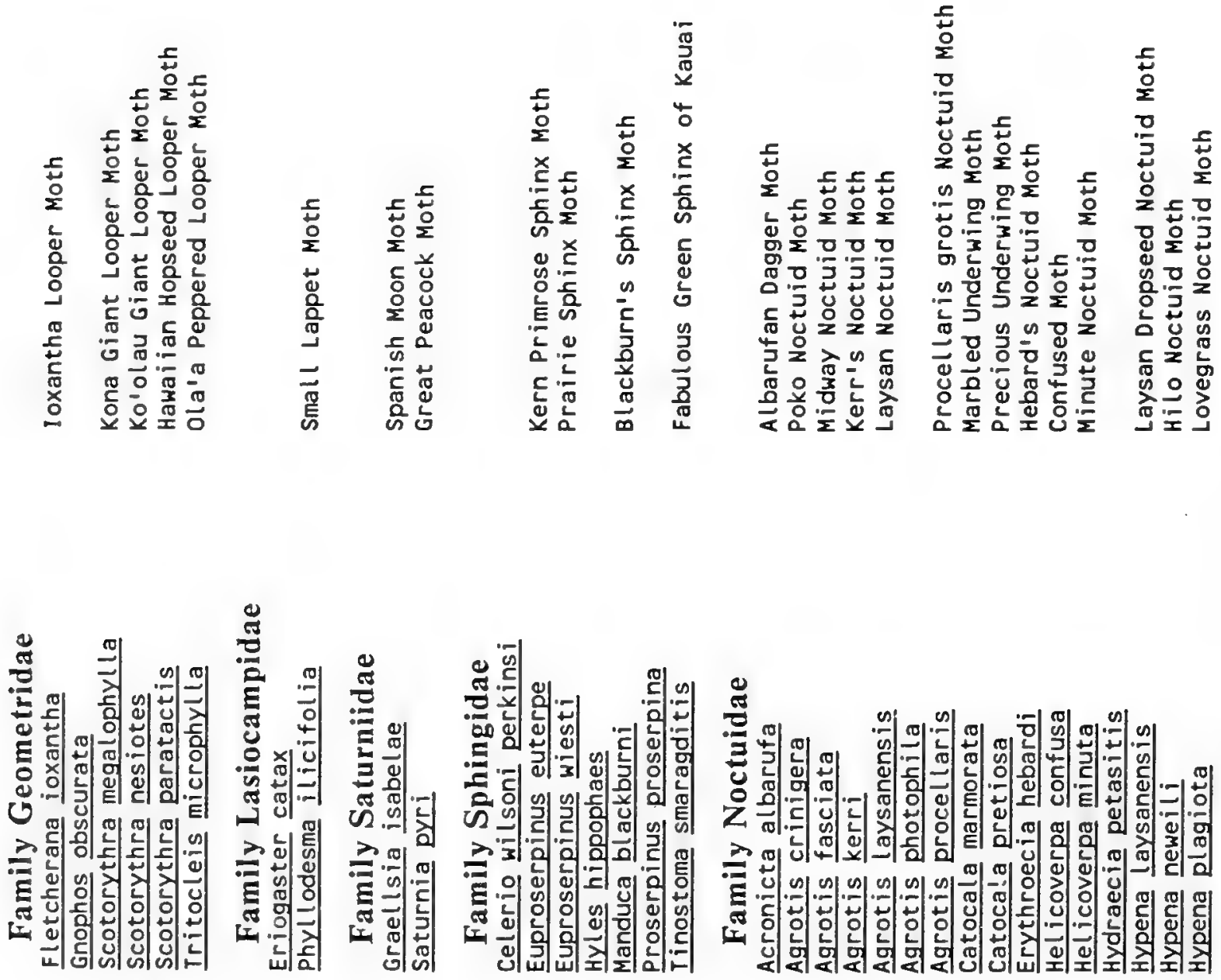

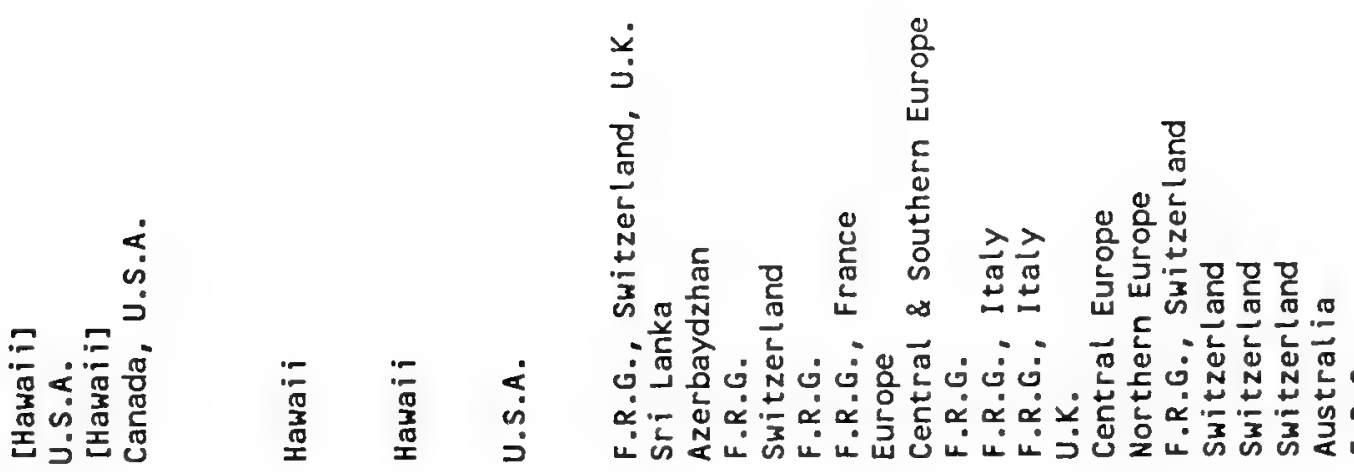

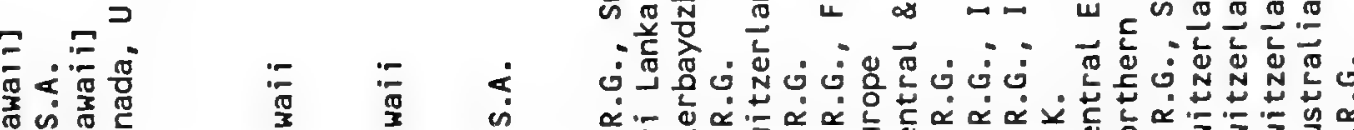
虽

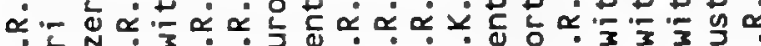

离一层-

\begin{tabular}{|c|c|c|c|c|c|c|c|c|c|c|}
\hline 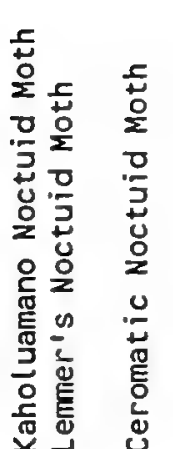 & 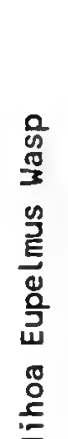 & 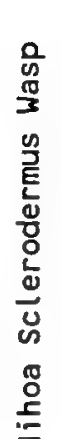 & 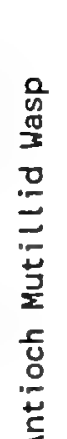 & 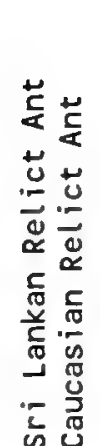 & 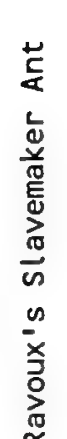 & 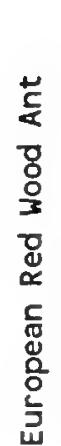 & 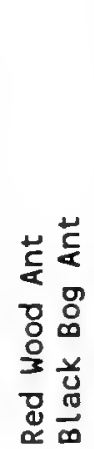 & 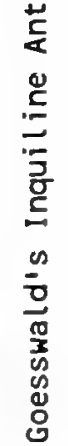 & 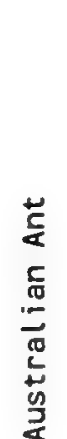 & 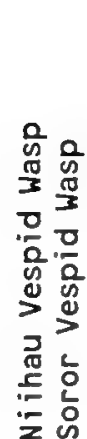 \\
\hline
\end{tabular}

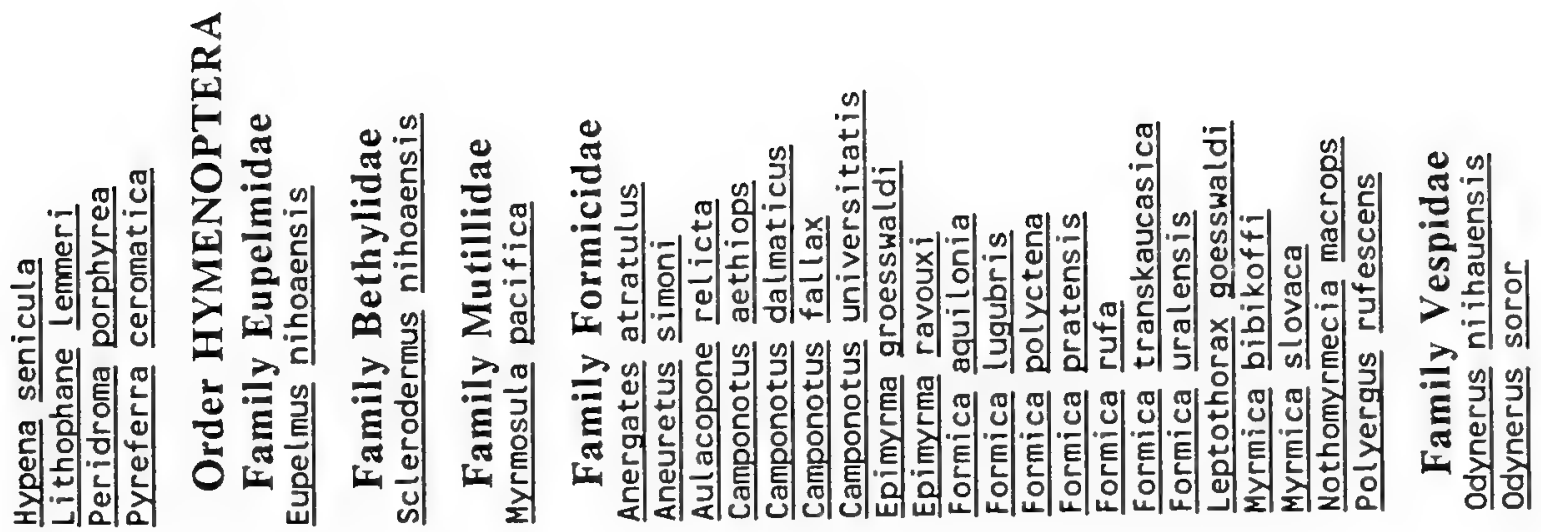




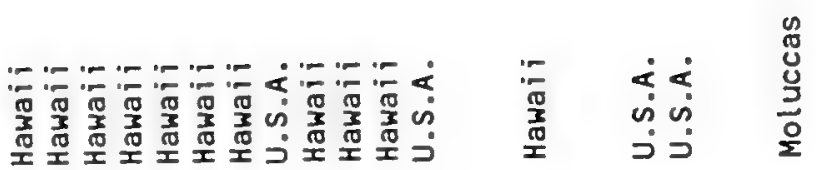

\&) 눈

응 웅

는

조

눈

总 苛

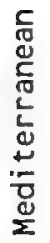

யน

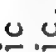

烍芹

峞茂

范苾

$\dot{1}$

焉

מש

$>>$

×它

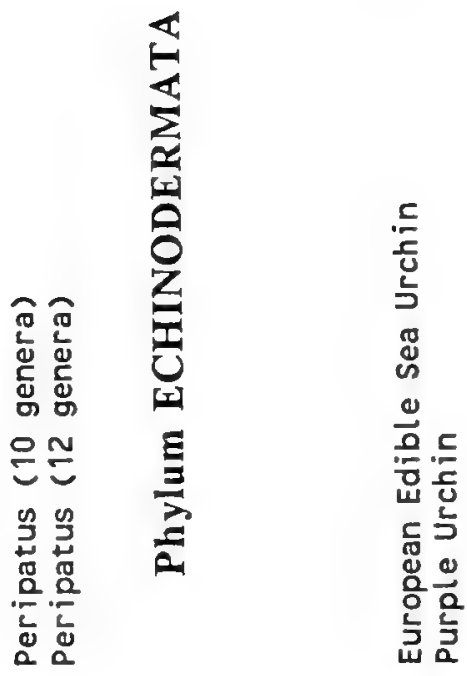

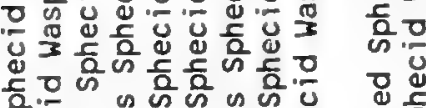

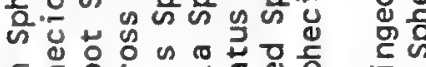

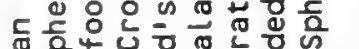

要造4心

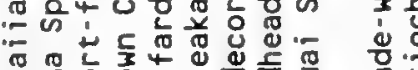

了.

$\mapsto \mapsto \quad x$

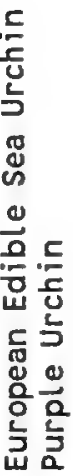

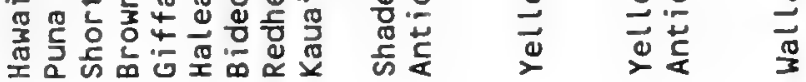
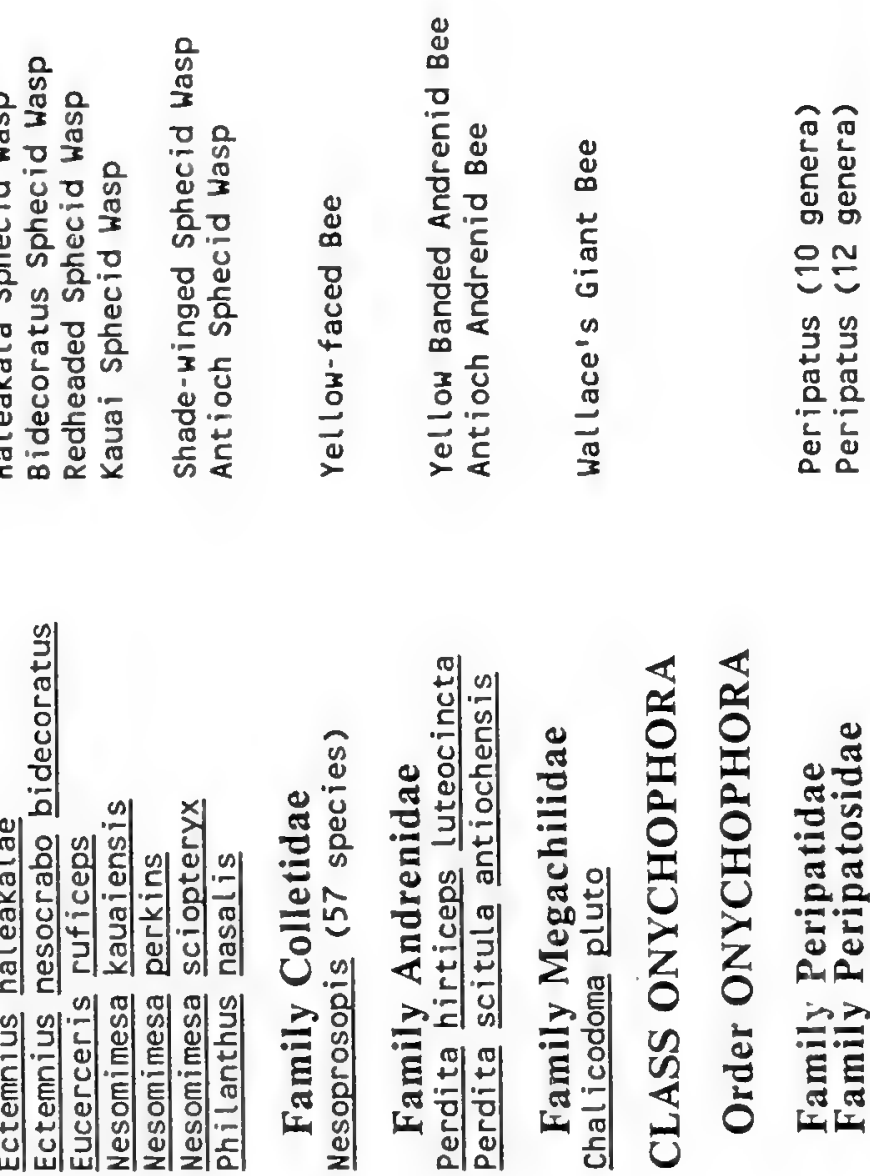

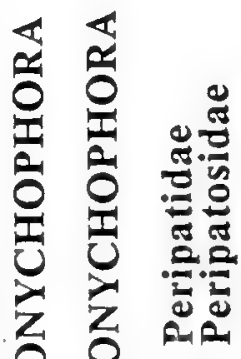

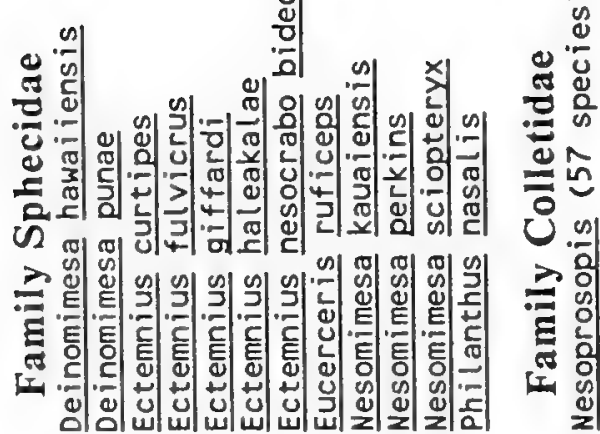

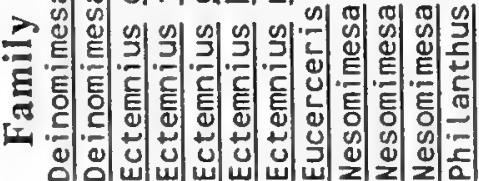

$\frac{2}{0}$

르를

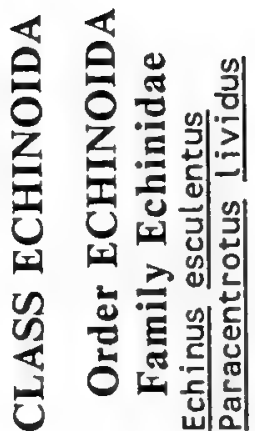


Aburria, 19

Acanthaclisis, 75

Acanthis, 31

Acanthodrilidae, 60

Acanthometropus, 66

Acanthorutilus, 40

Acavidae, 54

Accipiter, 18

Achatinella, 52

Achatinellidae, 52

Aciagrion, 67

Acinonyx, 11

Acipenser, 40

Acneus, 79

Acraea, 93

Acraeidae, 93

Acrantophis, 37

Acrididae, 72

Acrocephalus, 28

Acroceridae, 82

Acrolophitus, 72

Acronicta, 94

Actiniaria, 49

Addax, 14

Adelocosa, 61

Aegialia, 78

Aepyceros, 14

Aeschrithmysus, 80

Aeshna, 69

Aeshnidae, 69

Aethiothemis, 70

Agabus, 76

Agapetus, 83

Agapornis, 22

Agarodes, 84

Agelaius, 31

Agelastes, 19

Aglycyderidae, 81

Agonum, 76

Agriocnemis, 67

Agrotis, 94

Ailuropoda, 10

Akalats, 29

Alabamaubria, 79

Alaena, 88

Alasmidonta, 57

Alauda, 27

Albatrosses, 16

Alcelaphus, 14

Alethe, 28

Allanaspides, 62

Allenopithecus, 6

Alligator, 35

Allocebus, 5

Allocrangonyx, 63

Alloperla, 73

Allorhizucida, 70

Allotoca, 46
Aloeides, 88

Alouatta, 5

Amanipodagrion, 67

Amastridae, 53

Amauris, 90

Amaurocichla, 28

Amaurornis, 20

Amazona, 22

Amber Snail, 54

Ambloplites, 46

Amblyopsis, 44

Amblyrhynchus, 36

Amblysomus, 3

Ambrysus, 73

Ambystoma, 38

Ameiva, 36

Ameletus, 66

Ammocrypta, 47

Ammodorcas, 14

Ammodramus, 30

Ammonitella, 55

Ammonitellidae, 55

Ammopelmatus, 71

Ammotragus, 14

Amnicola, 50

Amphipoda, 63

Amphipods, 63

Amphispiza, 30

Amychus, 79

Amytornis, 28

Anaea, 92

Anagotus, 81

Anas, 17

Anaspidacea, 62

Anaspidae, 62

Anaspides, 62

Anax, 69

Ancylastrum, 52

Andrenidae, 96

Andrias, 38

Anectothemis, 70

Anergates, 95

Anetia, 90

Aneuretus, 95

Anguispira, 54

Annelida, 59

Anniella, 36

Anobiidae, 80

Anodorhynchus, 22

Anoglypta, 54

Anolis, 36

Anomala, 78

Anoplura, 73

Anostraca, 62

Anser, 17

Antanartia, 92

Anteaters, 7

Antechinus, 3
Antelopes, 14

Anthicidae, 80

Anthicus, 80

Anthipathidae, 49

Anthozoa, 49

Anthreptes, 30

Anthus, 27

Antilocapra, 14

Antipatharia, 49

Antipodochlora, 69

Antipodogomphus, 68

Antiponemertes, 50

Antpittas, 26

Antrolana, 62

Ants, 95

Antwrens, 26

Apalis, 28

Apalopteron, 30

Apatalestea, 82

Apatania, 83

Apaturopsis, 92

Apes, 7

Aphaostracon, 50

Aphodius, 78

Apiocertidae, 82

Aplonis, 32

Aplothorax, 76

Apodemia, 92

Appalachia, 72

Apterocychus, 77

Apteromantis, 70

Apus, 24

Aquila, 18

Ara, 28

Arachnida, 61

Araneae, 61

Araneidae, 61

Arapaima, 40

Aratinga, 22

Archaeogastropoda, 50

Arctocephalus, 11

Ardea, 17

Ardeotis, 21

Argiagrion, 67

Argonemertes, 50

Argyresthia, 85

Argyresthiidae, 85

Argyrocupha, 88

Arhynchobdellae, 60

Arionidae, 54

Arkansia, 57

Armadillos, 7

Armatobalanus, 62

Artamus, 32

Artemia, 62

Artemiidae, 62

Arthropoda, 60

Artogeia, 88
Arubolana, 62

Asaphomyia, 82

Asauridae, 61

Ascalaphidae, 75

Asellidae, 63

Ashmunella, 55

Asilidae, 82

Asio, 24

Aspiduchus, 70

Asses, 12

Assiminea, 51

Assimineidae, 51

Astacidae, 65

Astacoides, 65

Astacopsis, 65

Astacus, 65

Asteiidae, 83

Ataeniobiu:, 46

Ataenius, 78

Ateles, 5

Atelocynus, 10

Atelornis, 25

Athearni, 51

Athene, 24

Atlantisia, 20

Atlapetes, 30

Atractelmis, 79

Atrichornis, 26

Atrophaneura, 86

Atyidae, 65

Aulacopone, 95

Austrocordulia, 69

Austrolestes, 67

Austropotamobius, 65

Avahi, 5

Axis, 13

Axolotl, 38

Aythya, 17

Babblers, 29

Babyrousa, 13

Baetica, 71

Baetidae, 66

Balaena, 9

Balaenoptera, 9

Balanidae, 62

Balanus, 62

Balea, 54

Bandicoot, 3

Banksula, 62

Banza, 71

Barbs, 40

Barbus, 40

Baronia, 86

Basommatophora, 52

Bass, 46

Batagur, 33

Bathmocercus, 28 
Batomys, 8

Batrachophrynus, 39

Batrachoseps, 38

Bats, 4

Battus, 86

Bears, 10

Bees, 96

Beetles, 76

Behningiidae, 66

Belenois, 88

Belocephalus, 71

Beloneuria, 73

Belontia, 48

Belostoma, 73

Belostomatidae, 73

Bethylidae, 95

Bettongia, 2

Bhutanitis, 86

Binneya, 54

Birdwings, 86

Birgus, 66

Bivalvia, 57

Blackbirds, 30, 31

Blastocerus, 13

Blattaria, 70

Blattidae, 70

Blephariceridae, 82

Blindcats, 43

Blister Beetles, 80

Boas, 37

Bolborhynchus, 23

Boloria, 92

Bolyeria, 37

Bos, 14

Bostrychia, 17

Bothrops, 37

Bowdleria, 28

Brachycercus, 67

Brachylophus, 36

Brachypelma, 61

Brachypteracias, 25

Brachyteles, 5

Brachythemis, 70

Brachytron, 69

Bradypterus, 28

Bradypus, 7

Branchinecta, 62

Branchinectidae, 62

Branta, 17

Brennania, 82

Bristlebirds, 28

Bristletails, 66

Brvania, 83

Brychius, 76

Bubalus, 14

Bubo, 24

Budorcas, 14

Bufo, 39
Bugs, 73

Bulimulidae, 54

Bulimulus, 54

Bunolagus, 8

Buntings, 30

Buprestidae, 79

Buprestis, 79

Bush cricket, 71

Bustards, 21

Buteo, 18

Butterflies, 85

Cacajao, 5

Caconemobius, 71

Caddisflies, 83

Caecidotea, 63

Caenidae, 67

Caiman, 35

Caimans, 35

Cairina, 17

Callaeas, 32

Callagur, 33

Callicebus, 5

Callimico, 5

Callithrix, 5

Callophrys, 88

Caloenas, 21

Caloprymnus, 2

Calopterygidae, 68

Calopteryx, 68

Calosoma, 76

Calyptura, 26

Camaenidae, 55

Camarhynchus, 30

Cambaridae, 65

Cambarus, 65

Camels, 13

Camelus, 13

Campellolebias, 44

Campephaga, 27

Campephilus, 25

Camponotus, 95

Campsicnemus, 82

Canis, 10

Canthyria, 57

Capnia, 72

Capniidae, 72

Capra, 14

Capricornis, 14

Caprimulgus, 24

Caprolagus, 8

Capromys, 9

Capys, 88

Carabidae, 76

Carabus, 76

Carcinoscorpius, 60

Carelia, 53

Caretta, 34
Carettochelys, 34

Carolella, 85

Carposinidae, 85

Carterocephalus, 85

Carunculina, 57

Casarea, 37

Caseolus, 56

Catabasis, 43

Catagonus, 13

Catf ish, 43

Catinella, 54

Catocala, 94

Catostomus, 42

Catreus, 19

Cats, 11

Cattle, 14

Cavaticovelia, 73

Cavef ish, 44

Celerio, 94

Cephalophus, 14

Cephalopterus, 26

Ceraclea, 84

Cerambycidae, 80

Cerambyx, 80

Ceratella, 67

Ceratotherium, 12

Cercocebus, 6

Cercopithecus, 6

Cercyonis, 92

Ceriagrion, 67

Cervus, 13

Cesonia, 61

Chaeropus, 3

Chaetarthria, 77

Chaetomys, 8

Chalicodoma, 96

Channa, 48

Characodon, 46

Charadrius, 21

Charaxes, 92

Charina, 37

Charonia, 52

Chasmistes, 42

Chela, 40

Chelicerata, 60

Chelonia, 34

Cherax, 65

Chersodromia, 82

Chilota, 60

Chimpanzees, 7

Chinchilla, 9

Chioglossa, 38

Chiropotes, 5

Chlamyphorus, 7

Chloaeltis, 72

Chloephaga, 17

Chlorocichla, 27

Chlorolestidae, 67
Chloroperlidae, 73

Chloropeta, 28

Choeropsis, 13

Chondrohierax, 18

Chrysocyon, 10

Chrysomelidae, 81

Chrysoritis, 88

Chrysospalax, 3

Chub, 41

Cicadas, 75

Cicadellidae, 75

Cicadidae, 75

Cichlasoma, 48

Cichlherminia, 28

Cicindela, 76

Cicindelidae, 76

Ciconia, 17

Ciliophora, 49

Cincinnatia, 50

Cinclocerthia, 28

Cinclus, 28

Cirolanidae, 62

Cisco, 44

Cisticola, 28

Cistothorus, 28

Civets, 10

Cixiidae, 74

Clams, 59

Clamyphorus, 7

Claravis, 21

Clarias, 43

Clausiliidae, 54

Clavicoccus, 75

Clemmys, 33

Click Beetles, 79

Cnidaria, 49

Coahuilix, 50

Cobras, 37

Cochliopa, 51

Cochliopina, 51

Cochylidae, 85

Coeligena, 24

Coelus, 80

Coenagrion, 68

Coenagrionidae, 67

Coenobitidae, 66

Coenocorpyha, 21

Coenonympha, 91

Coleophora, 85

Coleophoridae, 85

Coleoptera, 76

Colinus, 19

Colletidae, 96

Collocalia, 24

Colobus, 6

Columba, 21

Conch, 52

Condors, 18 
Congothemis, 70

Conilurus, 8

Conocephaloides, 71

Conolophus, 36

Conradilla, 57

Conraua, 39

Coots, 20

Cophura, 82

Copris, 78

Copsychus, 28

Coracina, 27

Coracopsis, 23

Coralliidae, 49

Corallium, 49

Corals, 49

Corambidae, 52

Cordulegaster, 69

Cordulegasteridae, 69

Corduliidae, 69

Coregonus, 44

Coreobagrus, 43

Cornigomphus, 68

Corvus, 33

Cossypha, 28

Cotinga, 26

Cottus, 46

Coturnicops, 20

Coua, 23

Crabs, 66

Cranes, 20

Crangonyctidae, 63

Crangonyx, 63

Craseonycteris, 4

Crax, 19

Crayfish, 65

Creadion, 32

Creepers, 31

Crenichthys, 44

Crickets, 71

Criniger, 27

Crocidura, 3

Crocodiles, 35

Crocodylus, 35

Crossidius, 80

Crossleyia, 28

Crossoptilon, 19

Crotalus, 37

Crustacea, 62

Cruptochia, 83

Cruptoprocta, 10

Crupturellus, 15

Ctenizidae, 61

Cualac, 44

Cuckoos, 23

Cucujidae, 80

Cucujus, 80

Cumberlandia, 59

Cuon, 10
Curculionidae, 81

Cuscus, 2

Cyanolimnas, 20

Cyanopsitta, 23

Cyanoramphus, 23

Cyclocephala, 78

Cyclocosimia, 61

Cycloptilum, 72

Cvclura, 36

Cuclyrius, 88

Cylloepus, 79

Cymatiidae, 52

Cymbiodyta, 77

Cymothoe, 92

Cynolebias, 45

Cynomys, 8

Cyprinodon, 45

Cyprogenia, 57

Cyrtodactylus, 35

Cyrtonaias, 57

Cyrtopeltis, 74

Dace, 41

Daihinibaenetes, 71

Dalla, 85

Dama, 13

Damaliscus, 14

Damselflies, 67

Danaidae, 90

Danaus, 90

Darters, 47

Dasyornis, 28

Daubentonia, 5

Decapoda, 65

Deer, 13

Deinacrida, 71

Deinocossonus, 81

Deinomimesa, 96

Deloneura, 88

Delphacidae, 74

Dendrocopos, 25

Dendrocygna, 17

Dendroica, 31

Dendrolagus, 2

Dendroleon, 75

Dermaptera, 72

Dermatemys, 33

Dermochelys, 34

Desmana, 3

Desmocerus, 80

Desmopachria, 76

Deudorix, 88

Diastole, 55

Diceros, 12

Dicranopselaphus, 79

Dicrurus, 32

Didermocerus, 12

Didunculus, 22
Diomedea, 16

Dionda, 40

Diplomystes, 43

Diplotrema, 60

Dipluridae, 61

Dipodomys, 8

Diptera, 82

Discoglossus, 39

Discula, 56

Discus, 54

Distoleon, 75

Diving Beetles, 76

Dogs, 10

Dolania, 66

Doleschallia, 92

Dolichopodidae, 82

Dolomedes, 61

Dolophilodes, 84

Dolphins, 9

Donacia, 81

Dorcatragus, 14

Dorcopsis, 2

Dorcopsulus, 2

Dorcus, 77

Doridella, 52

Dragonflies, 68

Drepanoptila, 22

Dromus, 57

Drosophila, 83

Drosophilidae, 83

Drymarchon, 37

Dryobius, 80

Dryocichloides, 29

Dryococelus, 72

Dryocopus, 25

Dryolimnas, 20

Dryophthorus, 81

Dryotribus, 81

Dubiraphia, 79

Ducks, 17

Ducula, 22

Dugong, 12

Dune Beetles, 80

Durangonella, 51

Durbania, 88

Dusicyon, 10

Dynastes, 78

Dysticheus, 81

Dytiscidae, 76

Dytiscus, 76

Eagles, 18

Eanus, 79

Earthworms, 60

Earwigs, 72

Ecchiorolestes, 67

Ecclisomyja, 84

Echidnas, 2
Echinidae, 96

Echinodermata, 96

Echinoida, 96

Echinus, 96

Echymipera, 3

Ectemnius, 96

Ectodemia, 84

Edwardsiidae, 49

Edwardsina, 82

Efferia, 82

Egretta, 17

Eidoleon, 75

Elaphrus, 76

Elateridae, 79

Elephants, 12

Elephas, 12

Eleuthemis, 68

Eleutherodactylus, 39

Elliptio, 57

Elmidae, 79

Elona, 57

Elonidae, 57

Emerita, 66

Empetrichthys, 45

Empicoris, 74

Empididae, 82

Enallagma, 68

Endodontidae, 54

Engaewa, 65

Enopla, 50

Eopenthes, 79

Epargyreus, 85

Ephemera, 67

Ephemerella, 67

Ephemerellidae, 67

Ephemeridae, 67

Ephemeroptera, 66

Ephydridae, 83

Epicrates, 37

Epimyrma, 95

Epioblasma, 57

Epiophlebia, 68

Epiophlebiidae, 68

Equus, 12

Erebia, 91

Eremichthys, 41

Eremomela, 29

Eresidae, 61

Eresus, 61

Eretmochelys, 34

Erikssonia, 88

Eriogaster, 94

Erithacus, 29

Erpetogomphus, 68

Erymnochelys, 34

Erythroecia, 94

Erythrura, 32

Estrilda, 32 
Etheostoma, 47

Eubalaena, 9

Eucerceris, 96

Euchloe, 88

Eucnemidae, 79

Eucrenonaspides, 62

Eucyclogobius, 48

Eulidia, 24

Eulonchus, 82

Eumaeus, 88

Eumastacidae, 72

Eumorsea, 72

Eunice, 59

Eunicella, 49

Eunicida, 59

Eunicidae, 59

Eunymphicus, 23

Eupelmidae, 95

Eupelmus, 95

Euphydryas, 92

Eupleres, 10

Euploea, 90

Euproserpinus, 94

Eurycea, 38

Euryphura, 92

Eurystomus, 24

Eurytides, 86

Euscheman, 85

Eusthenia, 73

Eustheniidae, 73

Euthalia, 92

Eutriorchis, 18

Euxanthe, 92

Evarra, 41

Everes, 88

Fabriciana, 93

Falco, 18

Falcons, 18

Farula, 84

Felexamia, 75

Felis, 11

Ferminia, 28

Finchs, 30

Fireflies, 80

Fisherola, 52

Fletcherana, 94

Flies, 82

Flufftails, 20

Fluminicola, 51

Flycatchers, 29

Flying Foxes, 4

Fontelicella, 51

Fonticella, 51

Formica, 95

Formicidae, 95

Formicivora, 26

Fossa, 10
Foudia, 32

Francolins, 19

Francolinus, 19

Fregata, 16

Fringilla, 31

Frogs, 38

Fulica, 20

Fundulus, 45

Fusconaia, 58

Galaxias, 44

Galemys, 3

Galidictis, 10

Gallicolumba, 22

Gallinula, 20

Gallotia, 36

Gambelia, 36

Gambusia, 46

Gammaridae, 63

Gammarus, 63

Garter snakes, 37

Gastropoda, 50

Gavialis, 35

Gazella, 15

Gazelles, 15

Geckos, 35

Gelechiidae, 85

Geocapromys, 9

Geochelone, 33

Geomalacus, 54

Geometridae, 94

Geomitra, 56

Geonemertes, 50

Geopsittacus, 23

Geospiza, 30

Geothlypis, 31

Geotrupidae, 78

Gephryoglanis, 43

Geronticus, 17

Gerrhonotus, 36

Gharials, 35

Gibbons, 7

Gila, 41

Glacicavicola, 76

Glaresis, 78

Glaucidium, 24

Glaucis, 24

Glaucopsyche, 88

Glossosomatidae, 83

Glyphalinia, 55

Glyphopsyche, 84

Gnaphosidae, 61

Gnophos, 94

Gobies, 48

Gomphidae, 68

Gomphus, 68

Gongylomorphus, 36

Goniobasis, 51
Goodeids, 46

Gopherus, 34

Gorgonacea, 49

Gorilla, 7

Gorillas, 7

Gracillariidae, 85

Graellsia, 94

Grallaria, 26

Graphium, 86

Graphoderus, 76

Grapholitha, 85

Grasshoppers, 72

Grayling, 43

Grebes, 15

Greenbuls, 27

Gripopterygidae, 73

Gronocarus, 78

Ground Beetles, 76

Grus, 20

Gryllidae, 71

Grylloblatta, 71

Grylloblattaria, 71

Grylloblattidae, 71

Gryllotalpa, 71

Gryllotalpidae, 71

Guans, 19

Gulella, 54

Gulls, 21

Gymnobelideus, 2

Gymnocharacinus, 43

Gymnocthebius, 77

Gymnogyps, 18

Gymnopholus, 81

Gymnosomata, 52

Gyps, 18

Gyrinidae, 77

Hadramphus, 81

Haematopinidae, 73

Haematopinus, 73

Haematopus, 21

Haideoporus, 76

Halcyon, 24

Haliaeetus, 18

Haliplidae, 76

Haliplus, 76

Hansonoperla, 73

Hapalemur, 4

Hapalopsittaca, 23

Haplotaxida, 60

Hares, 8

Harpia, 18

Hawks, 18

Hedylepta, 93

Helicarionidae, 55

Helicidae, 56

Helicodiscidae, 55

Helicodiscus, 55
Heliconius, 93

Helicoverpa, 94

Helisoma, 52

Helix, 56

Helminthoglypta, 56

Helminthoglyptidae, 56

Heloderma, 36

Hemerobiidae, 75

Hemiargus, 88

Hemignathus, 31

Hemigomphus, 69

Hemiphaga, 22

Hemiphlebia, 67

Hemiphlebiidae, 67

Hemiptera, 73

Hemistena, 58

Hemitragus, 15

Hemitremia, 41

Heosemys, 33

Hepialidae, 84

Heptageniidae, 66

Hermits, 24

Herons, 17

Hesperia, 85

Hesperiidae, 85

Heteramphus, 81

Heterelmis, 79

Heterexis, 81

Heterocleon, 66

Heterocrossa, 85

Heteromirafra, 27

Heterotrichida, 49

Himantopus, 21

Hippidae, 66

Hippocamelus, 13

Hippopotami, 13

Hippopus, 59

Hipposideros, 4

Hippotragus, 15

Hirudinidae, 60

Hirudinoidea, 60

Hirudo, 60

Hirundo, 27

Holcobius, 80

Homarus, 65

Homoeoneuria, 66

Homoptera, 74

Honeyeaters, 30

Hoplonemertea, 50

Horseshoe crabs, 60

Huleechius, 79

Humblotia, 29

Huso, 40

Hutias, 9

Hyaena, II

Hybopsis, 41

Hydraecia, 94

Hydraena, 77 
Hydraenidae, 77

Hydrobiidae, 50

Hydrochara, 77

Hydrodamalis, 12

Hydromantes, 38

Hydrophilidae, 77

Hydroporus, 76

Hydroptila, 83

Hydrosaurus, 36

Hydrotilidae, 83

Hygrotus, 77

Hyla, 39

Hyles, 94

Hylobates, 7

Hymenoptera, 95

Hypena, 94

Hyperoodon, 9

Hypodryas, 93

Hypolimnas, 92

Hypsipetes, 27

Ibis, 17

Icaricia, 88

Ictalurus, 43

Ictinogomphus, 69

Idea, 90

Ideopsis, 91

Idiostatus, 71

Iguanas, 36

Indophlebia, 69

Indri, 5

Insecta, 66

Io, 51

Iotichthys, 41

Ischnura, 68

Isomma, 69

Isonychia, 66

Isopoda, 62

Isopods, 62

Ithmamar, 74

Ithonidae, 75

Itodacnus, 79

Jasus, 65

Kalania, 74

Kalochelidon, 27

Kangaroos, 2

Katechonemertes, 50

Katydids, 71

Kedestes, 85

Kenkia, 49

Kenkiidae, 49

Killifish, 45

Kinosternon, 33

Kites, 18

Kiwaia, 85

Kobus, 15
Komarekiona, 60

Komarekionidae, 60

Konia, 48

Koonunga, 62

Koonungidae, 62

Kosswigichthys, 45

Kupea, 93

Labeo, 41

Labidura, 72

Labiduridae, 72

Lagochila, 42

Lagorchestes, 2

Lagostrophus, 2

Lagothrix, 5

Lampetra, 39

Lampreys, 39

Lampsilis, 58

Lampyridae, 80

Lancidae, 52

Land Snails, 55

Lanius, 27

Lapwings, 21

Larks, 27

Larus, 21

Lasiocampidae, 94

Lasiorhinus, 2

Lasiurus, 4

Lasmigona, 58

Leaf Bugs, 74

Lednia, 72

Leeches, 60

Leiodidae, 77

Leiolopisma, 36

Leiopelma, 38

Leiostyla, 53

Lemur, 4

Lentipes, 48

Leontopithecus, 6

Lepidocephalus, 42

Lepidochelys, 34

Lepidochrysops, 89

Lepidomeda, 41

Lepidoptera, 84

Lepidostoma, 84

Lepidostomatidae, 84

Lepilemur, 4

Leporillus, 8

Leptobotia, 42

Leptoceridae, 84

Leptodea, 58

Leptogryllus, 72

Leptonemertes, 50

Leptoperla, 73

Leptothorax, 95

Leptotila, 22

Leptoxis, 51

Lestidae, 67
Lethe, 92

Leto, 84

Leucopeza, 31

Leucopsar, 32

Leucopternis, 18

Leucorrhinia, 70

Leuctra, 72

Leuctridae, 72

Lexingtonia, 58

Libelloides, 75

Libellula, 70

Libellulidae, 70

Libellulosoma, 69

Libythea, 90

Libytheidae, 90

Lice, 73

Lichnanthe, 78

Limnebius, 77

Limnephilus, 84

Limnodromus 21

Limulidae, 60

Limulus, 60

Linyphiidae, 61

Lioptilus, 29

Lipotes, 9

Lirceus, 63

Lithasia, 51

Lithoglyphus, 51

Lithophane, 95

Lizards, 35

Lobsters, 65

Longhorn Beetles, 80

Lophophrus, 19

Lophura, 19

Loxioides, 31

Loxodonta, 12

Loxops, 31

Lucania, 45

Lucanidae, 77

Luehdorfia, 86

Lutodrilidae, 60

Lutodrilus, 60

Lutra, 10

Lycaeides, 89

Lycaena, 89

Lycaenidae, 88

Lycaon, 10

Lycosa, 61

Lycosidae, 61

Lygaeidae, 74

Lymnaea, 52

Lymnaeidae, 52

Lyperobius, 81

Lytta, 80

Macaca, 6

Machiloides, 66

Macrancylus, 81
Macrobaenetes, 71

Macrobanetes, 71

Macrocephalon, 18

Macroderma, 4

Macrodontia, 80

Macrogalidia, 10

Macromia, 70

Macromiidae, 70

Macropus, 2

Macroscincus, 36

Macrosphenus, 29

Macrothele, 61

Macrotis, 8

Macroveliidae, 73

Maculinea, 89

Madtoms, 43

Magicicada, 75

Malacochers'ss, 34

Malaconotus, 27

Malimbus, 32

Malpulutta, 48

Manatees, 12

Manduca, 94

Mantidae, 70

Mantispa, 75

Mantispidae, 75

Mantodea, 70

Margaritifera, 59

Margaritiferidae, 59

Margaronia, 93

Marmota, 8

Marstonia, 51

Martins, 27

Masticophis, 37

Mayflies, 66

Mecistogaster, 68

Meda, 41

Megachilidae, 96

Megacolabus, 81

Megalagrion, 68

Megalonaias, 58

Mega!urus, 29

Megapodagrionidae, 67

Megapodes, 18

Megapodius, 18

Megaptera, 9

Megascolecidae, 60

Megascolides, 60

Megathymidae, 86

Megupsilon, 45

Melaenornis, 29

Melamprosops, 31

Melanerpes, 25

Melanosuchus, 35

Melignomon, 25

Meliphaga, 30

Meloidae, 80

Melospiza, 30 
Melursus, 10

Menidia, 46

Mergus, 17

Merostomata, 60

Merulaxis, 26

Merycomyia, 82

Mesitornis, 20

Mesodon, 55

Mesogastropoda, 50

Mesoveliidae, 73

Meta, 61

Metabolus, 29

Metacnemis, 67

Metapogon, 82

Metatrichoniscoides, 63

Metisella, 85

Metrarga, 74

Mexilana, 62

Mexipyrgus, 51

Mexistenasellus, 63

Mexithauma, 51

Mice, 8

Micrarionta, 56

Micraspides, 62

Microcebus, 5

Microchaetidae, 60

Microchaetus, 60

Microcreagris, 61

Microcryptorhynchus,

Microcylloepus, 79

Micronaspis, 80

Micropanchax, 45

Micropterus, 46

Microscolex, 60

Microtus, 8

Miloderes, 81

Minnows, 40

Mirafra, 27

Miridae, 74

Mistichthys, 48

Mixogaster, 82

Moapa, 41

Modulatrix, 29

Moho, 30

Mollusca, 50

Monachus, 11

Monadenia, 56

Monardithemis, 70

Mongooses, 10

Monias, 20

Monkeys, 5

Monticola, 29

Morimus, 80

Mormolyce, 76

Morphnus, 18

Mortonagrion, 68

Moschus, 13

Moss Beetles, 77
Moth Flies, 82

Moths, 84

Moxostoma, 42

Mudminnows, 43

Muntiacus, 14

Muscicapa, 29

Mussels, 57

Mustela, 10

Mutillidae, 95

Myadestes, 29

Myaka, 48

Mycotrupes, 78

Mycteria, 17

Mylothris, 88

63 Myotis, 4

Myrmecobius, 3

Myrmecophaga, 7

Myrmeleon, 75

Myrmeleontidae, 75

Myrmica, 95

Myrmosula, 95

Myrmotherula, 26

Myxas, 52

Naja, 37

Nannopterum, 16

Nasalis, 6

Naucorid Bugs, 73

8 Naucoridae, 73

Nectarinia, 30

Nectophrynoides, 39

Necydalis, 80

Neduba, 71

Nematostella, 49

Nemertea, 50

Nemertine Worms, 50

Nemopalpus, 82

Nemosia, 30

Nemouridae, 72

Nemours, 72

Neobisiidae, 61

Neochanna, 44

Neodrepanis, 26

Neofelis, 11

Neomorphus, 23

Neophema, 23

Neospiza, 30

Neothramma, 84

Neotragus, 15

Neotrichia, 83

Nephropidae, 65

Nephrops, 65

Nepticulidae, 84

Neptis, 93

Nerodia, 36

Neseis, 74

Nesidiolestes, 74

Nesillas, 29
Nesithmysus, 80

Nesocichla, 29

Nesocryptias, 74

Nesoenas, 22

Nesolagus, 8

Nesomantis, 39

Nesomimesa, 96

Nesomimus, 28

Nesoprosophis, 96

Nesorestias, 74

Nesosydne, 74

Nesothauma, 75

Nesotocus, 81

Netta, 17

Neurocordulia, 69

Neuroptera, 75

Newtonia, 29

Nicrophorus, 77

Ninox, 24

Nipponia, 17

Noctuidae, 94

Noeneura, 67

Nososticta, 67

Notarthrinus, 89

Nothaldonis, 81

Nothomyrmecia, 95

Notiomystis, 30

Notomys, 8

Notopterus, 40

Notornis, 20

Notropis, 41

Noturus, 43

Novumbra, 43

Numenius, 21

Nuthatchs, 30

Nymphalidae, 92

Nymphophilus, 51

Nysius, 74

Obovaria, 58

Oceanides, 74

Ochrotrichia, 83

Ochthebius, 77

Oclandius, 81

Odobenus, 11

Odocoileus, 14

Odonata, 67

Odontoceridae, 84

Odontophorus, 19

Odynerus, 95

Oecanthus, 72

Decetis, 84

Oedemasylus, 81

Oedura, 35

Oeoblia, 93

Ogmodon, 37

Ognorhynchus, 23

Oliarces, 75
Oliarus, 74

Oligochaeta, 60

Oligoneuridae, 66

Oligophlebodes, 84

Onchobarus, 81

Onthophagus, 78

Onychogalea, 2

Onychophora, 96

Oodemas, 81

Ophiogomphus, 69

Opiliones, 62

Optioservus, 79

Oravelia, 73

Orchestiidae, 64

Orconectes, 65

Oreodaimon, 42

Oreohelicidae, 55

Oreohelix, 55

Oreolyce, 89

Oreophasis, 19

Orestias, 45

Ornipholidotos, 89

Ornithoptera, 86

Ortalis, 19

Orthetrum, 70

Orthoptera, 71

Oryctes, 78

Oryx, 15

Oryzomys, 8

Osmerus, 44

Osmoderma, 78

Osteolaemus, 35

Otters, 10

Otus, 24

Ovis, 15

Owls, 23

Oxychaeta, 89

Oxyethira, 83

Oxygastra, 69

Oxyloma, 54

Oystercatchers, 21

Oysters, 57

Ozotoceros, 14

Pachycoccyx, 23

Pacifastacus, 65

Paddlefish, 40

Paduniella,

Palaemonetes, 65

Palaemonias, 65

Palaemonidae, 65

Paleoxenus, 79

Palinuridae, 66

Palmeria, 31

Palpopleura, 70

Paludiscala, 51

Pan, 7

Panchala, 89 
Panda, 10

Pangasianodon, 43

Pangasius, 43

Panoquina, 85

Pantagenia, 67

Panthera, 11

Pantinonemertes, 50

Panulirus, 66

Papilio, 87

Papilionidae, 86

Papio, 7

Papustyla, 55

Paracentrotus, 96

Paracoenia, 83

Paracymus, 77

Paradoxornis, 29

Parakeets, 23

Paranaspides, 62

Parantica, 91

Parapinnixa, 66

Pararge, 92

Parastacidae, 65

Paravitrea, 55

Pardosa, 61

Pareudiastes, 20

Parides, 87

Parnassius, 87

Paroreomya, 31

Parrotfinches, 22

Parrots, 22

Partula, 53

Partulidae, 53

Partulina, 52

Paryphanta, 54

Pavo, 19

Peafowls, 19

Pearlfish, 45

Pegias, 58

Pelecanus, 16

Pelobates, 39

Pelocoris, 73

Peltoperlidae, 73

Peltotrupes, 78

Penelope, 19

Pentalagus, 8

Pentarthrum, 81

Perameles, 3

Perca, 47

Percina, 47

Perdicella, 53

Perdita, 96

Perdix, 19

Peridroma, 95

Peripatidae, 96

Peripatosidae, 96

Peripatus, 96

Perlidae, 73

Petrels, 16
Petrochroa, 85

Petrogale, 2

Petroica, 29

Pezoparus, 23

Phaeognathus, 38

Phaeornis, 29

Phaethornis, 24

Phalacrocorax, 16

Phalanger, 2

Phalangodidae, 62

Phalanta, 93

Phallostethus, 46

Phaner, 5

Pharomacrus, 24

Phascogale, 3

Phasmatidae, 72

Phasmatoptera, 72

Pheasants, 19

Phelsuma, 35

Phenacobius, 42

Philanthus, 96

Phildoria, 85

Philocasca, 84

Philopotamidae, 83

Philoria, 39

Philotiella, 89

Phoca, 11

Phocoena, 9

Phodilus, 23

Photuris, 80

Phoxinus, 42

Phrynops, 34

Phrynosoma, 36

Phyciodes, 93

Phyllastrephus, 27

Phyllococcus, 75

Phyllodesma, 94

Phylolestes, 70

Physa, 52

Physidae, 52

Picathartes, 29

Picoides, 25

Picus, 25

Pieridae, 88

Pieris, 88

Pigeons, 21

Pinctada, 57

Pinnotheridae, 66

Pipile, 19

Pisidium, 59

Pithecophaga, 18

Pitta, 26

Pitymys, 8

Placostylus, 54

Plagiodontia, 9

Plagithmysus, 80

Plagopterus, 42

Planaria, 49
Planorbidae, 52

Planthoppers, 74

Platanista, 9

Platemys, 33

Platycnemididae, 67

Platycnemis, 67

Platyhelminthes, 49

Platysteira, 29

Plebejus, 89

Plebicula, 89

Plecoptera, 72

Plecotus, 4

Plethobasus, 58

Plethodon, 38

Pleurobema, 58

Pleuroceridae, 51

Plexauridae, 49

Ploceus, 32

Plovers, 21

Podarcis, 36

Podiceps, 15

Podilymbus, 16

Podocnemis, 34

Podogymnura, 3

Poeciliopsis, 46

Poecilmitis, 89

Pogonichthys, 42

Pogoniulus, 25

Polposipus, 80

Polychaeta, 59

Polvergus, 95

Polygra, 55

Polygyridae, 55

Polygyriscus, 55

Polyhymenophorea, 49

Polylamina, 78

Polyodon, 40

Polyphylla, 78

Polyplectron, 19

Polystictus, 26

Pomarea, 29

Pongo, 7

Potamilus, 59

Potorous, 2

Presbytis, 7

Prietella, 43

Prinia, 29

Priodontes, 7

Prionailurus, 11

Prionops, 27

Pristoceuthophilus, 71

Probarbus, 42

Problema, 85

Procambarus, 65

Procellaria, 16

Procnias, 26

Procolobus, 7

Prodontria, 78
Progomphus, 69

Prolagus, 7

Propithecus, 5

Proserpinus, 94

Prosobonia, 21

Prosorhochmidae, 50

Proterhinus, 80

Proteus, 38

Protoneuridae, 67

Protoploea, 91

Protoptila, 83

Prototroctes, 43

Psammaspididae, 62

Psammobates, 34

Psephenidae, 79

Psephenus, 79

Psephotus, 23

Pseudacris, 39

Pseudemydura, 35

Pseudemys, 33

Pseudibis, 17

Pseudiolaus, 89

Pseudirion, 66

Pseudocalyptomena, 25

Pseudochelidon, 27

Pseudococcidae, 75

Pseudocopaeodes, 86

Pseudocotalpa, 78

Pseudomys, 8

Pseudonestor, 31

Pseudopsectra, 75

Pseudoscorpionida, 61

Pseudostigmatidae, 68

Psilotreta, 84

Psittacula, 23

Psittirostra, 31

Psophodes, 29

Psychodidae, 82

Psychomastix, 72

Pteriidae, 57

Pterioida, 57

Pterocnemia, 15

Pterodroma, 16

Pteronura, 10

Pteropus, 4

Ptilinopus, 22

Ptychocheilus, 42

Pudu, 14

Puffinus, 16

Pungu, 48

Pupfish, 45

Pupillidae, 53

Pygathrix, 7

Pyralidae, 93

Pyreferra, 95

Pyriglena, 26

Pyrrhula, 23

Python, 37 
Pyxis, 34

Quadrula, 59

Quails, 19

Quetzals, 24

Rabbits, 8

Rails, 20

Rallus, 20

Ramphocinclus, 28

Rana, 39

Raphiomydas, 82

Rasbora, 42

Rats, 8

Rattlesnakes, 37

Redfins, 40

Reduviidae, 74

Regulus, 29

Reithrodontomys, 8

Relictus, 42

Rhaphidophoridae, 71

Rheas, 15

Rheinardia, 19

Rheobatrachus, 39

Rhinichthys, 42

Rhinoceros, 12

Rhinoceros, 12

Rhinoclemmys, 33

Rhinopithecus, 7

Rhinoplax, 25

Rhopalid Bugs, 74

Rhopalidae, 74

Rhopornis, 26

Rhyacophila, 83

Rhyacophilidae, 83

Rhynchopsitta, 23

Rhuncogonus, 81

Rhynochetos, 20

Rhytididae, 54

Riekoperla, 73

Riffle Beetles, 79

Rivulus, 44

Roaches, 70

Robberflies, 82

Robins, 28

Rollers, 25

Romanichthys, 47

Romankenkius, 49

Romerolagus, 8

Rosalia, 81

Rostrhamus, 18

Rowettia, 31

Rukia, 30

Rupicapra, 15

Saga, 71

Saguinus, 6

Saimiri, 5
Salamanders, 38

Salamis, 93

Salanoia, 10

Salmo, 44

Salvelinus, 44

Samoana, 53

Sanzinia, 37

Sapheopipo, 25

Sarotherodon, 48

Sarothrura, 20

Sasakia, 93

Satan, 43

Saturnia, 94

Saturniidae, 94

Satyridae, 91

Satyrodes, 93

Saxicola, 29

Scaphirhynchus, 40

Scarab Beetles, 77

Scarabaeidae, 78

Scavenger Beetles, 77

Schoutedenapus, 24

Sciurus, 8

Sclerodermus, 95

Scleropages, 40

Scotopelia, 24

Scotorythra, 94

Sculpins, 46

Scytalopus, 26

Sea Anemones, 49

Sea Fans, 49

Sea Slugs, 52

Sea Urchins, 96

Seais, 11

Seed Bugs, 74

Segmentina, 52

Selenarctos, 10

Semnornis, 25

Semotilis, 42

Serica, 78

Sericostomatidae, 84

Serins, 31

Serinus, 31

Sesiidae, 85

Sheep, 14

Sheppardia, 29

Shiiimiaeoides, 89

Shiners, 41

Shrikes, 27

Shrimps, 62, 65

Siacella, 74

Sicydium, 48

Silphidae, 77

Simias, 7

Simpsonichthys, 45

Simpsoniconcha, 59

Siphlonuridae, 66

Sitta, 30
Skiffia, 46

Skinks, 36

Skippers, 85

Slugs, 54

Smelt, 44

Smerina, 93

Sminthopsis, 3

Snails, 50

Snipe, 21

Solenodon, 3

Soliperla, 73

Somatochlora, 69

Somatogyrus, 51

Sonorella, 56

Sooglossus, 39

Sosippus, 61

Spanglerogyrus, 76

Sparrows, 30

Speirops, 30

Spelaeorchestia, 64

Speocirolana, 62

Speoplatyrhinus, 44

Speothos, 10

Speyeria, 93

Sphaeriidae, 59

Sphaeroderus, 76

Sphaeromatidae, 63

Sphalloplana, 49

Spharagemon, 72

Sphecidae, 96

Sphenodon, 35

Spheterista, 85

Sphingidae, 94

Spiders, 61

Spindasis, 90

Spinedace, 41

Spinus, 31

Spizocorys, 27

Splittails, 42

Spongillaflies, 75

Sporophila, 31

Springfish, 44

Stactolaema, 25

Stag Beetles, 77

Stagnicola, 52

Stallingsia, 86

Starlings, 32

Stenasellidae, 63

Stenasellids, 63

Stenelmis, 79

Stenodus, 44

Stenopelmatidae, 71

Stenopelmatus, 71

Stenophysa, 52

Stenotrema, 55

Stenotrupis, 81

Stentor, 49

Stentoridae, 49
Sterkia, 53

Sterna, 21

Sternotherus, 34

Stick-insects, 72

Stilts, 21

Stiobia, 51

Stomatepia, 48

Stoneflies, 72

Stonemyia, 82

Storks, 17

Strepera, 32

Streptaxidae, 54

Streptopelia, 22

Strider Bugs, 73

Strigops, 23

Strombas, 52

Strombidae, 52

Strymon, 90

Sturgeon, 40

Stygionympha, 92

Stygobromus, 63

Stylommatophora, 52

Stypodon, 42

Styrulus, 69

Succineidae, 54

Suckers, 42

Sula, 16

Sunbirds, 30

Sus, 13

Swallows, 27,32

Swallowtails, 87

Swifts, 24

Swynnertonia, 29

Sympetrum, 70

Synanthedon, 85

Syncaris, 65

Synlestidae, 70

Syrichtus, 86

Syrmaticus, 19

Syrphidae, 82

Tabanid Flies, 82

Tabanidae, 82

Tachybaptus, 16

Tachycnemis, 39

Tachypleus, 60

Taeniopterygidae, 72

Taeniopteryx, 72

Tamarins, 6

Tanagers, 30

Tanakia, 42

Tangara, 31

Tapaculo, 26

Taphius, 52

Tapirs, 12

Tapirus, 12

Tarantulas, 60

Tarsius, 5 
Tasmanophlebia, 66

Tauraco, 23

Taurotragus, 15

Teinobasis, 68

Teinopalpus, 88

Telema, 61

Telemidae, 61

Tenebrionidae, 80

Terns, 21

Terpsiphone, 29

Terrapene, 33

Terrapins, 33

Testudo, 34

Tetrao, 19

Tetrigidae, 72

Tetrix, 72

Tettigidea, 72

Tettigoniidae, 71

Thaanumia, 53

Thamnophis, 37

Thaumatibis, 17

Thaumatodon, 54

Thaumatogryllus, 72

Theraphosidae, 61

Thermosphaeroma, 63

Thestor, 90

Thinornis, 21

Thoracica, 62

Thread Bugs, 74

Threnetes, 24

Threskiornis, 17

Thrushes, 29

Thylacinus, 3

Thysanura, 66

Tiaroga, 42

Tiger Beetles, 76

Tigrisoma, 17

Tinamou, 15

Tinamus, 15

Tinostoma, 94

Tiradelphe, 91

Tirumala, 91

Tischeria, 84

Tischeriidae, 84

Toads, 39

Tokudaia, 8

Tolypeutes, 7

Tomistoma, 35

Torrent Midges, 82

Torreornis, 31

Tortoises, 33

Tortricidae, 85

Totoaba, 47

Touit, 23

Toxolasma, 59

Tragopan, 19

Treader Bugs, 73

Tree Snails, 53
Tremarctos, 10

Tremblers, 28

Treron, 22

Triaenodes, 84

Trichechus, 12

Trichocichla, 29

Tricholimas, 20

Trichoniscidae, 63

Trichoptera, 83

Tricladida, 49

Tridacna, 59

Tridacnidae, 59

Trigonopelastes, 78

Trigonoscuta, 82

Trimenia, 90

Tringa, 21

Triodopsis, 55

Trionyx, 35

Trithemis, 70

Tritocleis, 94

Tritogenia, 60

Tritons, 52

Trogidae, 77

Troglodytes, 28

63 Trogloglanis, 43

Troglophantes, 61

Troides, 88

Trout, 44

Trox, 77

Tryonia, 51

Tsitana, 86

Tuatara, 35

Turacos, 23

Turbellaria, 49

Turbinidae, 50

Turbo, 50

Turdoides, 29

Turdus, 29

Turtles, 33

Tylognathus, 42

Tympanuchus, 19

Typhaeus, 78

Typhlatya, 65

Typhlomolge, 38

Typhlops, 37

Typhlotriton, 38

Tyto, 23

Udeina, 60

Umbrellabirds, 26

Unas, 82

Unionidae, 57

Unionoida, 57

Uniramia, 66

Uranothauma, 90

Uratelornis, 25

Urothemis, 70

Ursus, 10
Utabaenetes, 71

Valvata, 50

Valvatidae, 50

Vanellus, 21

Varanus, 37

Varecia, 4

Veneroida, 59

Vermivora, 31

Vertiginidae, 53

Vertigo, 53

Vespericola, 55

Vespidae, 95

Vicugna, 13

Vicunas, 13

Villosa, 59

Vini, 22

Vipera, 37

Vipers, 37

Viverra, 10

Voles, 8

Wallabies, 2

Warblers, 28, 31

Wasps, 95

Water Beetles, 76

Weavers, 32

Weevils, 81

Weta, 71

Whales, 9

Whipbirds, 29

Whirligig Beetles, 77

White-eyes, 30

Whitefish, 44

Williamsonia, 70

Wolves, 10

Woodpeckers, 25

Worms, 59

Wrens, 28

Xantusia, 35

Xenicus, 26

Xenoophorus, 46

Xenopirostris, 27

Xenopus, 38

Xeromys, 8

Xipholena, 26

Xiphophorus, 46

Xiphorhynchus, 25

Xiphosura, 60

Xylotoles, 81

Xyrauchen, 42

Yellowbrows, 28

Ypthima, 92

Zaglossus, 2

Zaitzeva, 79
Zalophus, 11

Zavattariornis, 33

Zebras, 12

Zelotypia, 84

Zonitidae, 55

Zoothera, 30

Zoraptera, 73

Zorotypidae, 73

Zorotypus, 73

Zosterops, 30

Zyzomys, 8 
$$
\text { . }
$$ 
